\title{
ELECTROPHYSIOLOGICAL CORRELATES AND PREDICTORS OF THE ANTIDEPRESSANT RESPONSE TO REPEATED KETAMINE INFUSIONS IN TREATMENT-RESISTANT DEPRESSION
}

\section{SARA DE LA SALLE}

Thesis submitted to the University of Ottawa in partial Fulfillment of the requirements for the

Doctorate in Experimental Psychology

\author{
School of Psychology \\ Faculty of Social Sciences \\ University of Ottawa
}

(C) Sara de la Salle, Ottawa, Canada, 2020 


\section{ABSTRACT}

Traditional antidepressants, which act on the serotonin, dopamine, and norepinephrine systems, require many weeks to produce a therapeutic effect and are not effective for every patient. A sub-anesthetic dose of the anesthetic agent ketamine, a glutamate N-methyl-Daspartate receptor antagonist, has been shown to produce a rapid and robust antidepressant effect in treatment-resistant major depressive disorder (MDD). As depressive symptoms typically return after one week following a single infusion, recent work has begun to focus on methods for prolonging the effects. Repeated infusions on a specific dosing schedule are being explored, however, the early identification of treatment responders and non-responders would be beneficial for optimized treatment selection within this population.

The mechanisms underlying ketamine's rapid effects conceivably involve the regulation of altered glutamatergic signaling in MDD, though this is not yet completely understood. Understanding of the central mechanisms mediating ketamine's rapid antidepressant effects may be increased through the use of non-invasive electroencephalographic measures, including resting electroencephalography (EEG) and the mismatch negativity $(\mathrm{MMN})$ event-related potential. These measures have been shown to be altered in depressed individuals and are sensitive to ketamine administration.

The primary objectives of this study were to 1) examine acute changes in EEG- and MMN-derived indices, immediately post- and two hours postinfusion, with a sub-anesthetic ketamine dose in comparison to an active placebo (midazolam), and 2) to examine their relationships with early and sustained antidepressant treatment response to ketamine within an eight week clinical trial involving three study phases. 
Ketamine decreased measures of scalp-level alpha and theta resting activity, immediately postinfusion, and increased gamma immediately and two hours postinfusion. An increase in source-localized anterior cingulate activity two hours postinfusion was also observed. Regarding the MMN, ketamine reduced frontal amplitudes as well as theta event-related oscillations and source-localized peak frontal generator activity. Measures of resting theta and change in gamma, as well as left frontal MMN amplitude, theta event-related oscillations, baseline left phase locking factor, and baseline right inferior temporal lobe activity were predictive of decreases in depressive symptoms at both early and sustained treatment time points. Alpha power was predictive of decrease in suicidal ideation, though the relationship with baseline and early change in symptoms was stronger.

These findings contribute to our understanding of the role of baseline and ketamineinduced changes in both resting and task-evoked electrophysiological measures, and may have the potential to act as non-invasive biomarkers of antidepressant response prediction to glutamatergic agents. 


\section{PREFACE}

This thesis is comprised of manuscripts (Chapters 2-3) which are in preparation for submission for publication in peer-reviewed journals, as well as a General Introduction (Chapter 1) and Discussion (Chapter 4). Certain modifications have been made to the manuscripts in order to avoid redundancy. The authorship of both manuscripts (Chapters 2-3) are: Sara de la Salle, Jennifer Phillips, Pierre Blier, and Verner Knott.

Sara de la Salle (B.Sc.) completed all electrophysiological recordings and data analyses, and prepared the manuscripts.

Jennifer Phillips (Ph. D.) was a research associate of the primary clinical trial and provided clinical outcome data for participants in the add-on electrophysiological study. She also provided feedback on manuscript drafts.

Pierre Blier (M.D., Ph.D.) was the principal investigator of the primary clinical trial, as well as one of the clinicians involved in patient assessment and treatment. He also provided feedback on manuscript drafts.

Verner Knott (Ph.D., C.Psych.) was the principal investigator of the electrophysiological work. He was involved in the electrophysiological study conceptualization and analyses, and assisted with editing the present work. 


\section{ACKNOWLEDGEMENTS}

First and foremost, I must give all of my gratitude to my thesis supervisor, Dr. Verner Knott, for his years of mentorship, support, encouragement, and for nurturing my scientific curiosity. His ability to create a fun and supportive environment was equaled by the exciting and innovative projects that we undertook throughout my time in the lab.

The heart of the lab was truly the colleagues-turned-friends. I'd like to thank Dr. Natalia Jaworska for all of her guidance, especially in her role as lab director, as well as everyone that assisted me with my EEG recordings, participant sessions, data analysis questions, and so much

more: Dylan, Danielle, Joëlle, Crystal, Dhrasti, Hayley, and Ashley. Special thanks to Molly and Emma for their friendship and emotional support in more recent times.

Thank you to my wonderful thesis committee, Dr. Andra Smith, Dr. Ken Campbell, and Dr. Charles Collin, who I've had the pleasure of knowing and interacting with as a student over the years.

This study would not have been possible without the dedication to depression research and collaborative spirit of Dr. Pierre Blier and his team (Dr. Jennifer Phillips, Wendy Fusee, Holly English, Maria Da Silva). I would like to sincerely thank my study participants, who volunteered for this EEG component during an undoubtedly difficult period of time in their lives.

Even though school and research monopolized much of my time, I've been lucky to have so many great friends who have encouraged me non-stop and who have provided necessary diversions when needed. Special thanks to Meagan, Greg, Camille, and Jon, for their curiosity and enthusiasm about my research. I'm also very glad to have met Melissa on the first day of our program orientation, and for the enduring friendship that resulted. 
Finally, I'd like to thank my parents, for their unwavering support. Most importantly, I'd like to thank Joel, Lianne, and Nick for their patience and encouragement, especially over the past few months. They were the guiding light for me at the end of this long journey.

\section{DEDICATION}

I would like to dedicate this thesis to the memory of my grandpa, a hard worker and lifelong learner. 


\section{LIST OF PUBLICATIONS}

List publications completed during my Ph.D. program.

Knott V, Wright N, Shah D, Baddeley A, Bowers H, de la Salle S, Labelle A. (2020). Change in the Neural Response to Auditory Deviance Following Cognitive Therapy for Hallucinations in Patients With Schizophrenia. Front Psychiatry, 11, 555.

de la Salle S, Jaworska N, Blier P, Smith D, Knott V. (2020). Using prefrontal and midline right frontal EEG-derived theta cordance and depressive symptoms to predict the differential response or remission to antidepressant treatment in major depressive disorder. Psychiatry Res Neuroimaging, 302, 111109.

Wolff A, de la Salle S, Sorgini A, Lynn E, Blier P, Knott V, Northoff G. (2019). Atypical Temporal Dynamics of Resting State Shapes Stimulus-Evoked Activity in Depression-An EEG Study on Rest-Stimulus Interaction. Front Psychiatry, 10, 719.

de la Salle S, Inyang L, Impey D, Smith D, Choueiry J, Nelson R, Heera J, Baddeley A, Ilivitsky V, Knott V. (2019). Acute separate and combined effects of cannabinoid and nicotinic receptor agonists on MMN-indexed auditory deviance detection in healthy humans. Pharmacol Biochem Behav, 184, 172739.

de la Salle S, Shah D, Choueiry J, Bowers H, McIntosh J, Ilivitsky V, Knott V. (2019). NMDA Receptor Antagonist Effects on Speech-Related Mismatch Negativity and Its Underlying Oscillatory and Source Activity in Healthy Humans. Front Pharmacol, 10, 455.

Jaworska N, de la Salle S, Ibrahim MH, Blier P, Knott V. Leveraging Machine Learning Approaches for Predicting Antidepressant Treatment Response Using Electroencephalography (EEG) and Clinical Data. Front Psychiatry, 9, 768.

Hadjis E, Hyde M, Choueiry J, Jaworska N, Nelson R, de la Salle S, Smith D, Aidelbaum $\mathrm{R}$, Knott V. Effect of GAD1 genotype status on auditory attention and acute nicotine administration in healthy volunteers. Hum Psychopharmacol, 34(1), e2684.

de la Salle S, Choueiry J, Shah D, Bowers H, McIntosh J, Ilivitsky V, Knott V. Effects of Ketamine on Resting-State EEG Activity and Their Relationship to Perceptual/Dissociative Symptoms in Healthy Humans. Front Pharmacol, 7, 348.

Impey D, de la Salle S, Baddeley A, Knott V. (2017). Effects of an NMDA antagonist on the auditory mismatch negativity response to transcranial direct current stimulation. $J$ Psychopharmacol, 31(5), 614-624.

Hyde M, Choueiry J, Smith D, de la Salle S, Nelson R, Impey D, Baddeley A, Aidelbaum R, Millar A, Knott V. (2016). Cholinergic modulation of auditory P3 event- related potentials as indexed by CHRNA4 and CHRNA7 genotype variation in healthy volunteers. Neurosci Lett, $623,36-41$.

Impey D, de la Salle S, Knott V. (2016). Assessment of anodal and cathodal transcranial direct current stimulation (tDCS) on MMN-indexed auditory sensory processing. Brain Cogn, $105,46-54$. 
Mohammadi M, Al-Azab F, Raahemi B, Richards G, Jaworska N, Smith D, de la Salle S, Blier P, Knott V. (2015). Data mining EEG signals in depression for their diagnostic value. $B M C$ Med Inform Decis Mak, 15, 108.

Bowers H, Smith D, de la Salle S, Choueiry J, Impey D, Philippe T, Dort H, Millar A, Daigle M, Albert PR, Beaudoin A, Knott V. (2015). COMT polymorphism modulates the resting-state EEG alpha oscillatory response to acute nicotine in male non- smokers. Genes Brain Behav, 14(6), 466-76.

Knott V, de la Salle S, Smith D, Choueiry J, Impey D, Smith M, Beaudry E, Saghir S, Ilivitsky V, Labelle A. (2015). Effects of acute CDP-choline treatment on resting state brain oscillations in healthy volunteers. Neurosci Lett, 591, 121-5.

Knott V, de la Salle S, Choueiry J, Impey D, Smith D, Smith M, Beaudry E, Saghir S, Ilivitsky V, Labelle A. (2015). Neurocognitive effects of acute choline supplementation in low, medium and high performer healthy volunteers. Pharmacol Biochem Behav, 131, 119-29.

Knott V, Smith D, de la Salle S, Impey D, Choueiry J, Beaudry E, Smith M, Saghir S, Ilivitsky V, Labelle A. (2014). CDP-choline: effects of the procholine supplement on sensory gating and executive function in healthy volunteers stratified for low, medium and high P50 suppression. J Psychopharmacol, 28(12), 1095-108.

Knott V, Impey D, Philippe T, Smith D, Choueiry J, de la Salle S, Dort H. (2014). Modulation of auditory deviance detection by acute nicotine is baseline and deviant dependent in healthy nonsmokers: a mismatch negativity study. Hum Psychopharmacol, 29(5), 446-58.

Knott V, Choueiry J, Dort H, Smith D, Impey D, de la Salle S, Philippe T. (2014). Baseline-dependent modulating effects of nicotine on voluntary and involuntary attention measured with brain event-related P3 potentials. Pharmacol Biochem Behav, 122, 107-17.

Knott V, de la Salle S, Smith D, Phillipe T, Dort H, Choueiry J, Impey D. (2013). Baseline dependency of nicotine's sensory gating actions: similarities and differences in low, medium and high P50 suppressors. J Psychopharmacol, 27(9), 790-800.

de la Salle S, Smith D, Choueiry J, Impey D, Philippe T, Dort H, Millar A, Albert P, Knott V. (2013). Effects of COMT genotype on sensory gating and its modulation by nicotine: Differences in low and high P50 suppressors. Neuroscience, 241, 147-56. 


\section{TABLE OF CONTENTS}

ABSTRACT

PREFACE IV IV

ACKNOWLEDGEMENTS V V V V V

LIST OF PUBLICATIONS VII

TABLE OF CONTENTS IX

LIST OF TABLES $\quad$ XII

LIST OF FIGURES

ABBREVIATIONS $\quad$ XV

1. CHAPTER 1: GENERAL INTRODUCTION 1

1.1 Overview of Thesis Objectives

1.2 Major Depression Disorder (MDD), Treatment-Resistance, and Suicidality 1

1.3 Treatment-Resistance and Current Pharmacological Treatments for MDD 3

1.4 Role of the Glutamate System in MDD

1.5 Ketamine as an Antidepressant Agent

1.6. Electroencephalography (EEG) and Event-related Potentials (ERPs) 8

1.7 The Use of EEG/ERPs in Clinical Populations 9

1.8. Prospective Neuroelectrophysiological Predictors of Ketamine's Antidepressant 10 Effects

1.8.1 Alpha Power and Asymmetry

1.8.2 Frontal Theta Power and Source-Localized Theta 11

$\begin{array}{ll}\text { 1.8.3 Gamma Power } & 13\end{array}$

$\begin{array}{ll}\text { 1.8.4 Delta and Beta Power } & 14\end{array}$

1.8.5 Mismatch Negativity (MMN) 14

1.9. Ketamine-Induced Effects in MDD and their Relationship to Symptom Changes 18

1.10 Objectives and Hypotheses

2. CHAPTER 2: Scalp- and Source-Localized Electrophysiological Correlates and 21

Predictors of the Antidepressant Response to Repeated Ketamine Infusions in

Treatment-Resistant Depression

2.1 Abstract

2.2 Introduction 23

2.2.1 Growing Burden of Major Depressive Disorder (MDD) 23

2.2.2 Rising Use of Ketamine as an Antidepressant Agent 23

2.2.3 Ketamine-Induced Electrophysiological Changes 25

2.2.4 Prospective EEG Predictors of Ketamine's Antidepressant Effects 27

$\begin{array}{ll}2.2 .5 \text { The Current Study } & 28\end{array}$

2.3 Methodology 29

2.3.1 Participants 29

2.3.2 Study Design 30

2.3.3 Drug Administrations 31

2.3.4 EEG Acquisition $\quad 32$

2.3.5 EEG Analysis 33

2.3.5.1 Scalp Surface-Level Power and Derived Measures 33

2.3.5.2 Theta Source-Localized ACC Activity 34

2.3.6 Clinical Outcomes and Dissociative Symptom Assessment 34

2.3.7 Statistical Analysis 36 
2.4 Results 37

2.4.1 Demographics, Clinical Outcome, and Dissociative Symptom Measures 37

2.4.2 Regional- and Frequency-specific Changes with Ketamine and 39 Midazolam

$\begin{array}{ll}\text { 2.4.2.1 Delta } & 39\end{array}$

2.4.2.2 Theta 40

2.4.2.2.1 Theta1 40

2.4.2.2.2 Theta2 40

2.4.2.2.3 Theta Total 41

2.4.2.2.4 Frontal Midline Theta 42

2.4.2.2.5 Theta Cordance (PF, MRF) 43

2.4.2.2.6 Theta ACC 44

2.4.2.3 Alpha 44

2.4.2.3.1 Alpha1 44

2.4.2.3.2 Alpha2 44

2.4.2.3.3 Alpha Total $\quad 45$

2.4.2.3.4 Alpha Asymmetry $\quad 45$

2.4.2.4 Beta 46

$\begin{array}{ll}\text { 2.4.2.5 Gamma } & 47\end{array}$

2.4.3 Relationship between Baseline and Ketamine-induced Changes in EEG $\quad 48$

Measures with Acute and Sustained Change in MADRS/SI

2.4.3.1 Baseline EEG and Changes in MADRS/SI 48

2.4.3.2 Ketamine-induced Changes in EEG and in MADRS/SI 59

2.4.3.3 Relationship between Ketamine-induced Changes in EEG and 52 in BPRS-P

2.4.4 Prediction of Acute and Sustained Response to Ketamine 52

2.4.5 Baseline EEG Differences and Ketamine-Induced Changes between $\quad 55$

Early (1d) and Sustained (end of Ph2) Treatment Responders

2.5 Discussion

2.6 Limitations

2.7 Conclusion

3. CHAPTER 3: Acute Subanesthetic Ketamine-Induced Effects on the Mismatch Negativity (MMN) and their Relationship to Early and Sustained Treatment Response in Major Depressive Disorder

3.1 Abstract

3.2 Introduction 70

3.2.1 Role of Glutamate in Treatment-Resistant Major Depressive Disorder $\quad 70$ (MDD)

3.2.2 Mismatch Negativity (MMN), N-Methyl-D-Aspartate (NMDA) 71

Receptor Function, and MDD

3.2.3 Use of MMN Measures to Examine Ketamine's Antidepressant Effects 73

3.2.4 The Current Study

$\begin{array}{ll}3.3 \text { Methodology } & 75\end{array}$

3.3.1 Participants $\quad 75$

3.3.2 Study Design $\quad 75$

$\begin{array}{ll}\text { 3.3.3 Drug Administrations } & 75\end{array}$ 
$\begin{array}{ll}\text { 3.3.4 EEG Acquisition } & 75\end{array}$

$\begin{array}{ll}3.3 .5 \text { Paradigm } & 76\end{array}$

3.3.6 MMN Analysis 77

3.3.7 Source-Localized Activity for MMN Generator Regions of Interest $\quad 78$ (ROIs)

3.3.8 Clinical Outcomes and Dissociative Symptom Assessment 80

3.3.9 Statistical Analysis $\quad 80$

3.4 Results 81

3.4.1 Demographics, Clinical Outcome, and Dissociative Symptom Measures $\quad 81$

3.4.2 Changes in MMN Measures with Ketamine and Midazolam $\quad 82$

3.4.2.1 Frontal Amplitude and Latency $\quad 82$

3.4.2.2 Temporal Amplitude and Latency $\quad 83$

3.4.2.3 N1 Amplitude and Latency $\quad 86$

3.4.2.4 Theta Event-Related Oscillations (ERO) and Phase Locking 87

Factor (PLF)

3.4.2.5 Source-localized MMN Peak ROIs $\quad 89$

3.4.3 Relationship between Ketamine-induced Changes in MMN and in $\quad 90$

BPRS-P

3.4.4 Relationship between Baseline and Ketamine-induced Changes and $\quad 90$

MADRS $\Delta 2 \mathrm{hrs}, \Delta 1 \mathrm{~d}, \Delta \mathrm{Ph} 2(\mathrm{~N}=23)$ and $\Delta \mathrm{Ph} 3(\mathrm{~N}=13)$ MADRS

3.4.5 Prediction of Acute and Sustained Response to Ketamine 92

3.5 Discussion

92

3.6 Limitations $\quad 95$

3.7 Conclusion $\quad 96$

4. CHAPTER 4: GENERAL DISCUSSION

4.1 Summary $\quad 97$

4.2. Ketamine's Effects on EEG-derived Measures $\quad 98$

4.3. Ketamine's Effects on MMN Indices 98

4.4. Use of EEG/MMN Indices for Treatment Prediction 99

4.5. Conclusion and Future Directions 103

REFERENCES

$\begin{array}{lr}\text { APPENDICES } & 127\end{array}$

Appendix I: NMDA Receptor Antagonist Effects on Speech-Related Mismatch 127

Negativity and its Underlying Oscillatory and Source Activity in Healthy Humans (publication)

Appendix II: Effects of Ketamine on Resting-State EEG activity and Their

Relationship to Perceptual/Dissociative Symptoms in Healthy Humans (publication)

Appendix III: Mini-International Neuropsychiatric Interview (MINI) 162

Appendix IV: Antidepressant Treatment History Form (ATHF) 189

Appendix V: Montgomery-Åsberg Depression Rating Scale (MADRS) 193

Appendix VI: Brief Psychiatric Rating Scale-Positive Symptoms subscale (BPRS-P) 194 


\section{LIST OF TABLES}

Table 2.1 Demographic features and clinical characteristics of participants $(\mathrm{N}=24)$. Means and standard deviations are presented.

Table 2.2 a. Changes in Montgomery-Åsberg Depression Rating Scale (MADRS), means \pm S.D.; b. Changes in Brief Psychiatric Rating Scale- Positive Symptoms (BPRS$\mathrm{P})$, means \pm S.D.

Table 2.3 Model parameters for regression analyses.

Table 2.4 Means ( \pm S.E.) for baseline variables and Mann-Whitney U-test and exact $p$ values for responders and non-responders at one day and Phase 2.

Table 3.1 Mean $( \pm$ S.E.) peak MMN frontal and temporal subcomponent amplitudes $(\mu \mathrm{V})$ and latencies (ms) at each time point and drug condition.

Table 3.2 Mean $( \pm$ S.E. $)$ peak N1 amplitudes $(\mu \mathrm{V})$ and latencies $(\mathrm{ms})$ at each time point and drug condition.

Table 3.3 Mean $( \pm$ S.E.) peak MMN frontal event-related oscillations $(\mu \mathrm{V})$ and phase locking factor (PLF) values at each time point and drug condition.

Table 3.4 Mean ( \pm S.E. $)$ ln-transformed source-localized CSD values $\left(\mathrm{A} / \mathrm{m}^{2}\right)$ of peak MMN generator activity.

Table 3.5 Significant correlations $(r, p)$ between baseline MMN measures with change in Montgomery-Åsberg Depression Rating Scale (MADRS) at 2 hours ( $\Delta 2 \mathrm{hrs})$ and one day $(\Delta 1 \mathrm{~d})$ postinfusion, and change from baseline to end of phase $2(\Delta \mathrm{Ph} 2)$ and end of phase $3(\Delta \mathrm{Ph} 3)$.

Table 3.6 Model parameters for regression analyses. 


\section{LIST OF FIGURES}

Figure 2.1 a. Timeline of three study phases and $\mathbf{b}$. timeline of EEG recording sessions of Phase 1.

Figure 2.2 Electrode recording montage and electrode groupings for analysis.

Figure 2.3 Anterior cingulate cortex (ACC) regions of interest (ROIs): sgACC (BA25), top; rACC (BA24), middle; dACC (BA32), bottom.

Figure 2.4 Average delta power $\left(\mu \mathrm{V}^{2}\right) \pm$ S.E. and topographical headmaps at each time point and drug condition. ${ }^{*}=$ time comparison, $\$=$ drug condition comparison, $\mathrm{p}<.05$.

Figure 2.5 Average theta power $\left(\mu \mathrm{V}^{2}\right) \pm$ S.E. and topographical headmaps at each time point and drug condition. ${ }^{*}=$ time comparison, $\$=$ drug condition comparison, $\mathrm{p}<.05$.

Figure 2.6 Average alpha power $\left(\mu \mathrm{V}^{2}\right) \pm$ S.E. and topographical headmaps at each time point and drug condition. ${ }^{*}=$ time comparison, $\$=$ drug condition comparison, $\mathrm{p}<.05$.

Figure 2.7 a. Average central-parietal beta power $\left(\mu \mathrm{V}^{2}\right)$ and topographical headmaps at each time point and drug condition and $\mathbf{b}$. Frontal (F3, F4) gamma spectral plots $\left(\mu \mathrm{V}^{2}\right) \pm$ S.E. ${ }^{*}=$ time comparison, $\$=$ drug condition comparison, $\mathrm{p}<.05$.

Figure 2.8 Scatterplots of significant correlations between baseline current source density (CSD) anterior cingulate cortex (ACC)-localized activity, frontal alpha asymmetry, and alpha total (AlphaT) parieto-occipital power $\left(\mu \mathrm{V}^{2}\right)$ with change in Montgomery-Åsberg Depression Rating Scale (MADRS) and MADRS item 10 (suicidal ideation, SI) at 2 hours ( $\Delta 2 \mathrm{hrs})$ and one day $(\Delta 1 \mathrm{~d})$ postinfusion, and change from baseline to end of phase $2(\Delta \mathrm{Ph} 2)$, and end of phase $3(\Delta \mathrm{Ph} 3)$.

Figure 2.9 Scatterplots of significant correlations between change in prefrontal (PF) theta cordance, frontal midline theta 1 (FMT1) power $\left(\mu \mathrm{V}^{2}\right)$, and frontal gamma power with change in Montgomery-Åsberg Depression Rating Scale (MADRS) and MADRS item 10 (suicidal ideation, SI) at 2 hours $(\Delta 2 \mathrm{hrs}$ ) and one day $(\Delta 1 \mathrm{~d})$ postinfusion, and change from baseline to end of phase $3(\Delta \mathrm{Ph} 3)$.

Figure 2.10 Scatterplots of significant correlations between change in alpha total (AlphaT) power $\left(\mu \mathrm{V}^{2}\right)$ with change in Montgomery-Åsberg Depression Rating Scale (MADRS) item 10 (suicidal ideation, SI) at 2 hours $(\Delta 2 \mathrm{hrs})$ and one day $(\Delta 1 \mathrm{~d})$ postinfusion, and change from baseline to end of phase $2(\Delta \mathrm{Ph} 2)$, and end of phase $3(\Delta \mathrm{Ph} 3)$.

Figure 2.11 Mean ( \pm S.E.) baseline EEG features that differed in responders and nonresponders at one day postinfusion and at the end of phase 2 . 
Figure 3.1 Electrode recording montage and electrode groupings (frontal=grey, mastoids=black) for analysis.

Figure 3.2 MMN temporal and frontal generator regions of interest (ROI) defined by (Waberski et al., 2001).

Figure 3.3 Peak MMN topographic maps at each time point and drug condition.

Figure 3.4 Grand averaged frontal (F3, left; Fz, midline; F4, right) and temporal (TP9, left; TP10, right) MMN difference waveforms at each time point and drug condition. $*=$ time comparison, $\mathrm{p}<.05$.

Figure 3.5 Frontal midline $(\mathrm{Fz})$ grand averaged N1 waveforms at each time point and drug condition. ${ }^{*}=$ time comparison, $\mathrm{p}<.05$.

Figure 3.6 Grand averaged time frequency plots showing theta (a) event-related oscillations $(\mu \mathrm{V})$ and $(\mathrm{b})$ phase-locking factor at the frontal midline $(\mathrm{Fz})$ site at each time point and drug condition.

Figure 3.7 Scatterplots of significant correlations between baseline and ketamine-induced changes in MMN measures with change in Montgomery-Åsberg Depression Rating Scale (MADRS) at 2 hours ( $\triangle 2 \mathrm{hrs}$ ) and one day $(\Delta 1 \mathrm{~d})$ postinfusion, and change from baseline to end of phase $2(\Delta \mathrm{Ph} 2)$ and end of phase $3(\Delta \mathrm{Ph} 3)$.

Figure 4.1 Summary of most predictive variables at the four time points ( $2 \mathrm{hrs}, 1 \mathrm{~d}, \mathrm{Ph} 2$, Ph3) for depressive and suicidal symptoms. $\Delta 2 \mathrm{hrs}=$ change from pre- to 2 hours postinfusion; $\Delta 1 \mathrm{~d}=$ change from pre- to 1 day postinfusion; $\Delta \mathrm{Ph} 2=$ change from baseline to end of phase $2 ; \Delta \mathrm{Ph} 3=$ change from baseline to end of phase 3; CSD = current source density; $\mathrm{ERO}=$ event-related oscillations; ITL = inferior temporal lobe; MADRS = Montgomery-Åsberg Depression Rating Scale; MADRS SI = Montgomery-Åsberg Depression Rating Scale item 10; $\mathrm{MMN}=$ mismatch negativity; $\mathrm{PF}=$ prefrontal; $\mathrm{PLF}=$ phase-locking factor; $\mathrm{r} / \mathrm{sgACC}=\mathrm{rostral} /$ subgenual anterior cingulate cortex .

Figure 4.2 Optimal use of EEG/MMN measures in the prediction of antidepressant treatment response. $\mathrm{r} / \mathrm{sgACC}=$ rostral/subgenual anterior cingulate cortex; $\mathrm{MMN}=$ mismatch negativity; PLF $=$ phase-locking factor; ITL = inferior temporal lobe; $\mathrm{CSD}=$ current source density; $\Delta 2 \mathrm{hrs}=$ change from pre- to 2 hours postinfusion. 


\begin{tabular}{|c|c|}
\hline \multicolumn{2}{|c|}{ ABBREVIATIONS } \\
\hline $\mathrm{ACC}$ & anterior cingulate cortex \\
\hline alphaT & alpha total \\
\hline AMPA & $\alpha$-amino-3-hydroxy-5-methyl-4-isoxazolepropionic acid \\
\hline ANOVA & analysis of variance \\
\hline BA & brodmann area \\
\hline BDNF & brain-derived neurotrophic factor \\
\hline BOLD & blood-oxygen-level-dependent \\
\hline CEN & central executive network \\
\hline CSD & current source density \\
\hline CNS & central nervous system \\
\hline $\mathrm{dACC}$ & dorsal anterior cingulate cortex \\
\hline DBS & deep brain stimulation \\
\hline $\mathrm{DMN}$ & default mode network \\
\hline DSM & diagnostic and statistical manual of mental disorders \\
\hline $\mathrm{ECT}$ & electroconvulsive therapy \\
\hline EEG & electroencephalography \\
\hline EOG & electrooculography \\
\hline ERO & event-related oscillations \\
\hline ERN & error-related negativity \\
\hline ERP & event-related potential \\
\hline eLORETA & exact low-resolution electromagnetic tomographic analysis \\
\hline FFT & fast fourier transform \\
\hline fMRI & functional magnetic resonance imaging \\
\hline FMT & frontal midline theta \\
\hline GABA & gamma-Aminobutyric acid \\
\hline HAMD & Hamilton depression rating scale \\
\hline HEOG & horizontal electrooculography \\
\hline${ }^{1} \mathrm{H}-\mathrm{MRS}$ & proton magnetic resonance spectroscopy \\
\hline HNK & (2R,6R)-hydroxynorketamine \\
\hline
\end{tabular}




\begin{tabular}{|c|c|}
\hline iSPOT-D & international Study to Predict Optimized Treatment in Depression \\
\hline i.v. & intravenous \\
\hline KET & ketamine \\
\hline LDAEP & loudness dependence of the auditory evoked potential \\
\hline 1STL & left superior temporal lobe \\
\hline LPP & late positive potential \\
\hline MADRS & Montgomery-Åsberg depression rating scale \\
\hline MDD & major depressive disorder \\
\hline MEG & magnetoencephalography \\
\hline MID & midazolam \\
\hline MINI & Mini International Neuropsychiatric Inventory \\
\hline MMN & mismatch negativity \\
\hline MNI & Montreal neurological institute \\
\hline MRF & midline right frontal \\
\hline MRI & magnetic resonance imaging \\
\hline $\mathrm{ms}$ & milliseconds \\
\hline mTOR & mammalian target of rapamycin \\
\hline NMDAR & N-Methyl-d-aspartate receptor \\
\hline PET & positron emission tomography \\
\hline $\mathrm{PF}$ & prefrontal \\
\hline PLF & phase-locking factor \\
\hline qEEG & quantitative electroencephalography \\
\hline $\mathrm{rACC}$ & rostral anterior cingulate cortex \\
\hline rITL & right inferior temporal lobe \\
\hline rSTL & right superior temporal lobe \\
\hline rmANOVA & repeated-measures analysis of variance \\
\hline ROI & region of interest \\
\hline rTMS & repetitive transcranial magnetic stimulation \\
\hline SD & standard deviation \\
\hline SE & standard error of the mean \\
\hline
\end{tabular}


sgACC subgenual anterior cingulate cortex

SI

suicidal ideation

eLORETA exact low resolution brain electromagnetic tomography

SNRI serotonin and norepinephrine reuptake inhibitors

SN

salience network

SSRI

selective serotonin re-uptake inhibitor

STAR*D

Sequenced Treatment Alternatives to Relieve Depression

TCA

tricyclic antidepressant

ThetaT

theta Total

TR

treatment resistant

VEOG

vertical electrooculography

VPP

vertex positive potential 


\section{CHAPTER 1: GENERAL INTRODUCTION}

\subsection{Overview of Thesis Objectives}

The aims of this work touch on three specific areas: 1) What neural changes occur with ketamine (vs. midazolam, an active placebo) immediately and two hours postinfusion in patients with treatment-resistant (TR) major depressive disorder (MDD), 2) how do these changes relate to decreases in depressive symptoms and suicidal ideation, and 3) are these acute changes predictive of both early and sustained response to ketamine as a treatment? These questions were explored within a multi-phase clinical ketamine trial examining acute and repeated subanesthetic ketamine infusions for TR MDD. Electrophysiological recordings were performed prior to, immediately post- and two hours postinfusion within the first randomized, placebocontrolled crossover phase of the trial. The acute ketamine-induced electroencephalography (EEG) and event-related potential (ERP) changes were quantified and examined in relation to changes in early and sustained treatment response.

\subsection{Major Depression Disorder (MDD), Treatment-Resistance, and Suicidality}

Major Depressive Disorder (MDD) is a serious health condition which is estimated to affect more than 264 million people throughout the world, and is the largest contributor to the global burden of disease (Smith, 2014; World Health Organization, 2017). It is generally more prevalent among females ( $\sim 5.1 \%$ vs. $\sim 3.6 \%$ in males) and tends to peak in older adulthood (World Health Organization, 2017). MDD can severely impair functioning (Hammer-Helmich et al., 2018) and can reduce an individual's lifespan (Cuijpers \& Schoevers, 2004).

A diagnosis of MDD is most commonly based on the Diagnostic and Statistical Manual of Mental Disorders, Fifth Edition (DSM-5), which requires the presence of depressed mood 
and/or a loss of interest or pleasure in nearly all activities for a period of at least two weeks, as well as another four of the following symptoms: changes in appetite or weight, sleep, and psychomotor activity (either increase or decrease), fatigue or decreased energy, feeling of worthlessness or guilt, decreased ability to concentrate or make decisions, and recurrent thoughts of death or suicidal ideation (American Psychiatric Association, 2013). MDD symptom presentations are extremely variable, and two patients with a DSM-5 diagnosis could not share a single symptom and receive the same diagnosis (Fried, 2017; Rush, 2007). A recent examining of 3703 depressed outpatients in the "Sequenced Treatment Alternatives to Relieve Depression" $\left(\mathrm{STAR}^{*} \mathrm{D}\right)$ study identified 1030 unique symptom profiles (Fried \& Nesse, 2015). This supports the heterogeneity of MDD and suggests the need for individualized approaches to diagnosis and treatment.

MDD is also highly recurrent (Burcusa \& Iacono, 2007). Following a first major depressive episode, there is a $60 \%$ lifetime risk of recurrence; this increases to $70 \%$ for those with a history of two episodes, and $90 \%$ for three or more episodes. Additionally, between one third and half of individuals with MDD relapse within a year following the end of treatment (Monroe \& Harkness, 2011). With each subsequent recurrence, the individual has an increased risk of developing treatment-resistance, functional impairment, comorbid health problems, and potential suicidality (Lye et al., 2020).

MDD is often comorbid with suicidal ideation (Nock et al., 2010), with a rate of suicide completion ranging from $2-10 \%$ in outpatient studies and $15 \%$ in inpatient studies (Angst et al., 1999; Nierenberg et al., 2001). However, a meta-analysis of longitudinal research found that current risk factors of suicidal thoughts and behaviours (ideation, attempts, suicide death) such as prior suicide ideation/attempts, psychiatric hospitalization, non-suicidal self-injury, hopelessness, 
depression and anxiety, were only slightly better than chance at predicting risk (Franklin et al., 2017). There is also an overall lack of research for short-term risk prediction (Glenn \& Nock, 2014; Klonsky et al., 2016). The current method of suicide risk assessment relies almost exclusively on self-report, which can be biased due to motivations to conceal intentions or feelings of shame and fear (Glenn \& Nock, 2014). Additionally, little is known regarding when and why suicidal ideation progresses to a suicide attempt (Klonsky et al., 2016).

\subsection{Treatment-Resistance and Current Pharmacological Treatments for MDD}

Most pharmacological treatments for MDD are rooted in the monoamine hypothesis of depression, which suggest that certain neurotransmitter deficits (i.e. serotonin, norepinephrine, and dopamine) are responsible for inducing the core features of depression (Hirschfeld, 2000). As such, the major pharmacological drug classes used in the treatment of MDD include selective serotonin reuptake inhibitors (SSRIs), serotonin-norepinephrine reuptake inhibitors (SNRIs), monoamine oxidase inhibitors, tricyclic antidepressants (TCA), and atypical antidepressants. These drugs are administered in order to increase monoamine neurotransmitter levels in the synaptic space in order to activate postsynaptic receptors (Hillhouse \& Porter, 2015). Many people $(\sim 1 / 3)$ who seek treatment for MDD often do not tolerate or respond to traditional pharmacotherapeutic treatments, and some $(\sim 1 / 3)$ only achieve a partial response (Kennedy et al., 2001; Rush et al., 2006). These individuals are considered to be "treatment-resistant" (TR). Although there is no universal definition of the term "treatment-resistant depression", it is most often used to describe an inadequate or lack of response to treatment (Kasper, 2014). There is also a lag time between treatment initiation and therapeutic response for antidepressant medications (Porcelli et al., 2011). Despite the fact that pharmacological effects begin within hours of drug administration, therapeutic effects only begin to manifest within two to four weeks 
from treatment initiation. This delay in depressive symptom reduction can be critical for the individual due to the possibility of symptom worsening and suicide risk (Ferrari \& Villa, 2017). The monoamine hypothesis lacks adequate explanations of this delayed onset of action of antidepressant medications and the lack of effect in this large subgroup of TR MDD (Sanacora et al., 2012).

\subsection{Role of the Glutamate System in MDD}

A growing body of research has suggested that abnormalities in the glutamate system may be implicated in the pathophysiology and treatment of depression, which has led to the formulation of the glutamate hypothesis of MDD (Sanacora et al., 2012). This hypothesis emerged from the observation that glutamate N-methyl-D-aspartate (NMDA) receptor antagonists exerted antidepressant-like effects (Trullas \& Skolnick, 1990).

Comparative studies of MDD vs. healthy controls have found abnormalities in glutamate and glutamine levels, including increased concentrations of glutamate in plasma, increased concentration of glutamine in cerebrospinal fluid, and higher post-mortem levels of glutamate in the frontal cortex of people with MDD (Hashimoto et al., 2007; Hillhouse \& Porter, 2015). Additionally, proton magnetic resonance spectroscopy $\left({ }^{1} \mathrm{H}-\mathrm{MRS}\right)$ studies have found both reduced glutamate/glutamine exchange in subcortical and cortical brain regions of MDD patients, as well as elevated concentrations of glutamine in the occipital cortex (Sanacora et al., 2014). Finally, there is also evidence for region-specific alterations in the expression of NMDA receptor subunits in MDD patients (Hillhouse \& Porter, 2015). These studies provide support for the investigation of the glutamate system as a target for treatment response in MDD. 


\subsection{Ketamine as an Antidepressant Agent}

Building on accumulating pre-clinical evidence suggesting the involvement of glutamate and NMDA receptors in the pathophysiology of MDD, a clinical study by Berman et al. (2000) involving ketamine, a potent non-competitive NMDA receptor antagonist, found confirmatory results in a small crossover study of sub-anesthetic ketamine versus saline in eight individuals with MDD. Ketamine, a phencyclidine derivative, has been used as an anesthetic agent in veterinary and human medicine since 1970 (Abdallah et al., 2015). While the exact mechanism through which ketamine exerts its antidepressant response remains unknown, the current hypothesis is that it increases glutamate neurotransmission in the prefrontal cortex, which leads to increased synaptic plasticity and neurotrophic signaling through downstream molecular cascades (Lener et al., 2017). Ketamine antagonizes NMDA receptors on inhibitory GABAergic interneurons, which disinhibits cortical glutamatergic neurons, thereby increasing glutamatergic signalling. It also antagonizes the post-synaptic NMDA receptors, which is thought to enhance glutamatergic signaling through $\alpha$-amino-3-hydroxy-5-methyl-4-isoxazolepropionic acid (AMPA) receptors. These effects are thought to increase the synthesis of brain-derived neurotrophic factor (BDNF) and the activity of the mammalian target of rapamycin (mTOR), leading to neuroplasticity and synaptogenesis (Feifel, 2016). Recent work has also pointed to one of ketamine's active metabolites (2R,6R)-hydroxynorketamine (HNK), which is able to produce antidepressant effects within animal studies, but is not an NMDA antagonist (Zanos et al., 2016).

In the first randomized, placebo-controlled study of ketamine's antidepressant effects, the antidepressant response manifested within a few hours, but was generally maximal after 24 hours. In addition to a rapid response (after 24 hours, $71 \%$ response), 35\% of individuals with treatment-resistant MDD maintained their clinical response for at least one week (Zarate et al., 
2006). In this example, as in most studies assessing an antidepressant treatment response, a "responder" is defined as an individual who exhibits a $50 \%$ or greater decrease in their depressive symptoms as measured by a depression rating scale such as the Hamilton Depression Rating Scale (HAMD) or the Montgomery-Åsberg Depression Rating Scale (MADRS). Since this first study, a single sub-anesthetic dose of ketamine has repeatedly been shown to exert rapid antidepressant effects in individuals with unipolar and bipolar depression (Bobo et al., 2016; Caddy et al., 2015). Depressive symptom reductions typically manifest within a few hours, with the effect peaking at 24 hours, and symptoms returning within one week postinfusion (Kishimoto et al., 2016). A recent meta-analysis also found that a single dose of ketamine was able to rapidly reduce suicidal ideation, and that these effects were partially independent of the antidepressant effect (Wilkinson et al., 2018a).

A limitation of earlier placebo-controlled ketamine trials was their lack of an effective placebo. Even low doses of ketamine $(0.2-0.5 \mathrm{mg} / \mathrm{kg})$ produce mild derealization and therefore the use of saline is inadequate as a control (Blier et al., 2012). A recent comparison with studies utilizing the benzodiazepine midazolam as an "active" placebo (Grunebaum et al., 2018; Murrough et al., 2015; Murrough et al., 2013) suggested that midazolam was superior to saline for blind integrity (Wilkinson et al., 2019). Remifentanil has also been used as an active placebo as it induces physiological and psychological side effects, produces no antidepressant effect, and has a short half-life ( $\sim 3-4$ mins; Sumner et al., 2020). Additionally, most studies have used a racemic mix of the $R$ - and $S$ - enantiomers, though recent studies have suggested that esketamine ( $S$-ketamine) is more potent and may be less likely to cause psychotomimetic or other adverse effects. An esketamine nasal spray was found to be effective as a rapid antidepressant treatment for MDD patients with active suicidal ideation with intent (Fu et al., 2020). 
As ketamine's rapid antidepressant properties have been confirmed numerous times since the early 2000s, improvements in blinding integrities have been developed, and new research avenues with esketamine and HNK are being pursued, the next frontier involves the prolonging on the antidepressant effect. Recent work has focused on specific dosing schedules (twiceweekly, thrice-weekly) aimed at prolonging the antidepressant effects (aan het Rot et al., 2010; Cusin et al., 2017; Murrough et al., 2013b; Phillips et al., 2019; Shiroma et al., 2014; Singh et al., 2016; Vande Voort et al., 2016; Wilkinson et al., 2018b).

Despite the fact that ketamine has rapid antidepressant properties, has a short half-life, and has relatively few side effects when administered sub-anesthetically without a bolus dose, being able to identify responders vs. non-responders with a high degree of certainty is important because some individuals may not initially respond to a single ketamine dose. Phillips et al. (2019) examined single, repeated (thrice-weekly for 2 weeks), and maintenance (once weekly, for a month) infusions, and found that repeated infusions led to cumulative and sustained reductions in depressive symptoms that were maintained in responders. In addition, nonresponders to single infusions responded to repeated infusions. This finding is extremely important in the context of TR MDD, as a potentially beneficial treatment may not be administered if an individual is not an early responder. If it can be known prior to treatment initiation or very early on that a certain individual is not likely to respond, they can immediately be offered an alternative treatment. The ultimate goal is to reduce prolonged periods of suffering on the part of each individual. To date, there have been no biological or genetic predictors of sufficient clinical utility to inform such a process (Labermaier et al., 2013). Consequently, much work remains to be carried out in order to better understand which individual will respond to ketamine, both initially and in a sustained manner. 


\subsection{Electroencephalography (EEG) and Event-related Potentials (ERPs)}

Increasing our understanding of the underlying neural alterations associated with ketamine's antidepressant effects can be achieved through the use of non-invasive measures of resting brain activity and early sensory processes, using electroencephalography (EEG) and event-related potentials (ERPs). Measured using electrodes placed on the scalp, EEG records the brain's spontaneous electrical activity (inhibitory or excitatory postsynaptic potentials from thousands of pyramidal cells) as the difference in voltage between two sites (Light et al., 2010). These signals are subject to modification by electrical conductive properties of brain tissues, of the electrode itself, and the position of the underlying cortical generator in relation to the recording electrode (Olejniczak, 2006). Spontaneous oscillations have been described in terms of their frequency: delta $(\sim 1-4 \mathrm{~Hz})$, theta $(\sim 4-8 \mathrm{~Hz})$, alpha $(\sim 8-13 \mathrm{~Hz})$, beta $(\sim 13-30 \mathrm{~Hz})$, and gamma (>30Hz; Alhaj et al., 2011; Schomer \& da Silva, 2010).

ERPs are small electrical potentials generated by the brain and time-locked in response to specific internal or external events (e.g., stimuli, responses, decisions). In a laboratory setting, multiple presentations of a stimulus are necessary to create an averaged signal for the quantification of amplitude $(\mu \mathrm{V})$ and latency (milliseconds, ms), which are purported to represent the extent of neural resource allocation and the time course of processing. This averaged ERP waveform consists of a series of positive and negative voltage components reflecting early automatic sensory processing, largely dependent on physical stimulus parameters, and later higher-order cognitive processing (Luck, 2012). The ability to assess both early and later sensory and cognitive processes with millisecond precision makes ERPs one of the most informative and dynamic methods for studying human sensory processing (Duncan et al., 2009). 


\subsection{The Use of EEG/ERPs in Clinical Populations}

As EEG and ERPs have been well-characterized in healthy human populations, specific deviations have become the focus of study in many clinical populations. These alterations have led researchers to make interferences regarding the nature and locus of brain dysfunction (Duncan et al., 2009) and have been increasingly examined for potential diagnostic and prognostic purposes (Hajcak et al., 2019; Popa et al., 2020). EEG and ERPs are also particularly well-suited for the examination of the effects of pharmacological substances on the central nervous system (CNS), a sub-discipline known as "pharmaco-EEG". Pharmaco-EEG is primarily used for the purposes of indicating CNS penetration/absorption and dynamic activity and monitoring potential drug toxicity (e.g. excessive sedation, induction of epileptiform activity), but it can also be used in the study of psychotropic treatment response prediction (Mucci et al., 2006).

The use of quantitative EEG (qEEG) and ERPs have shown promise in detecting dysfunction in MDD. These measures, obtained either prior to or soon after treatment initiation, have been found to predict subsequent individual clinical response to antidepressants (Alhaj et al., 2011). As such, the use of these direct brain-based methods of assessing underlying depressive pathology and its response to treatment could prove to be a valuable tool in depression pharmacotherapy. In clinical practice, an effective biomarker must be reliable, reproducible, cost effective, and non-invasive (Holsboer, 2008). EEG is relatively inexpensive, completely non-invasive, and has been shown to demonstrate high levels of split-half and test-

retest reliability (Light et al., 2010; Thatcher, 2010). Therefore, the continued study of its use for treatment response prediction in MDD is imperative. 


\subsection{Prospective Neuroelectrophysiological Predictors of Ketamine's Antidepressant Effects}

Several measures derived from resting (i.e. participant sitting still with their eyes closed in a relaxed but wakeful state) EEG recordings have been described in the MDD literature. The most predictive of the proposed measures involve the theta (power, cordance, source-localized anterior cingulate $[\mathrm{ACC}]$ activity) and alpha (power, asymmetry) frequency bands (Iosifescu, 2011; Schiller, 2019).

\subsubsection{Alpha Power and Asymmetry}

Alpha $(\sim 8-13 \mathrm{~Hz})$ is the dominant oscillation in the human brain during wake, most evident during a relaxed but wakeful state (Zoon et al., 2013), though it has also been implicated in various cognitive processes, including attention, perception, and working memory, and may prioritize relevant sensory input (Klimesch, 1999, 2012; Van Diepen et al., 2019). It is both heritable (Smit et al., 2005) and temporally stable over the course of months and years (Näpflin et al., 2007; Tenke et al., 2018). It has been found to be inversely related to the brain's state of activation and arousal, and has also been suggested as an index of relative cortical inhibition/deactivation (Klimesch, 2012; Klimesch et al., 2007; Neuper \& Pfurtscheller, 2001), as it is inversely related to blood-oxygen-level dependent (BOLD) signals (Laufs et al., 2003) and cortical excitability (Lange et al., 2013).

Individuals with MDD tend to exhibit greater alpha power (i.e. less activation) over right parietotemporal regions, potentially reflecting diminished emotional arousal (Heller \& Nitscke, 1997). They have also been found to exhibit higher left frontal alpha activity (i.e. left frontal hypoactivation) compared to healthy controls (Thibodeau et al., 2006). This frontal alpha 'asymmetry' has been framed within the approach-withdrawal hypothesis, in which higher left 
activity is related to approach motivation and higher right activity is related to avoidance/ withdrawal motivation (Harmon-Jones \& Gable, 2018). Alpha asymmetry has been proposed as a state-invariant prognostic biomarker (van der Vinne et al., 2017). Recent work has also indicated a relationship between suicidal ideation and alpha asymmetry (Park et al., 2019; Roh et al., 2020).

In terms of treatment prediction, elevated pre-treatment alpha power, most consistently in the posterior regions, as well as left alpha lateralization, have been shown to differentiate responders and non-responders to TCAs and SSRIs (Bruder et al., 2001, 2008). Healthy control studies of sub-anesthetic doses of ketamine have consistently shown reductions in alpha power (de la Salle et al., 2016 [Appendix I]; Forsyth et al., 2018; Kochs et al., 1996; McMillan et al., 2019; Muthukumaraswamy et al., 2015; Vlisides et al., 2017, 2018), indicating an overall decrease in arousal and possible disruption in processing of salient information.

\subsubsection{Frontal Theta Power and Source-Localized Theta}

Theta $(4-8 \mathrm{~Hz})$ power is highest over the frontal midline region and has been associated with emotional and cognitive processes (attention, working memory, executive function) (Mitchell et al., 2008). Frontal theta band power is altered in MDD, with patients showing higher activity in the anterior and right hemisphere (Kwon et al., 1996; Ricardo-Garcell et al., 2009). This activity has been suggested to reflect altered activity in the anterior cingulate cortex (ACC), which has been implicated in affective processing (Jaworska et al., 2012). The direction of findings relating to pre- and early treatment prediction have produced inconsistent results, with studies showing both decreased and increased pre-treatment theta in responders, as well as reduced relative theta power at one week post-treatment (Iosifescu, 2011). In response to these mixed results, more sensitive measures of theta activity have been suggested. 
Using EEG source-localization methods, higher pre-treatment theta ACC activity was found to predict antidepressant treatment response (Pizzagalli, 2011; Pizzagalli et al., 2018). Theta cordance is a quantitative EEG measure which combines information derived from absolute $\left(\mu \mathrm{V}^{2}\right)$ and relative (\%) spectral power (Leuchter et al., 1999) and is derived from two main regions of interest: prefrontal (PF) and midline and right frontal (MRF). Decrease in theta cordance has been found in antidepressant treatment responders after one week and has consistently been found to be predictive of response to multiple treatment modalities (Bares et al., 2007, 2008; Broadway et al., 2012; Hunter et al., 2018). The MRF region was previously identified through a hierarchical cluster analysis demonstrating medication-specific changes (Leuchter et al., 2008). The PF region has been examined in more studies, though neither region has been found to be superior (de la Salle et al., 2020).

Theta power has shown differential ketamine-induced based on the administration of a bolus (i.e. a dose given over a short period of time) dose. A bolus dose is typically administered prior to a continuous infusion in order to induce psychotomimetic effects as a model for including schizophrenia-like symptoms in healthy controls (Beck et al., 2020). Increases in frontal theta $(4-8 \mathrm{~Hz})$ have been found in studies that employed a bolus dose (Forsyth et al., 2018; McMillan et al., 2019; Muthukumaraswamy et al., 2015), while those that did not have found decreases in theta power (de la Salle et al., 2016 [Appendix I]; Knott et al., 2006; Vlisides et al., 2017, 2018). PF theta cordance also decreases following ketamine infusion in healthy controls (Horacek et al., 2010; Sanacora et al., 2014). Horacek et al. (2010) proposed that acute ketamineinduced neurophysiological changes were similar to the gradual monoaminergic-based antidepressant changes, and that this change could serve as a marker and a predictor of the rapid antidepressant effect. 


\subsubsection{Gamma Power}

Though not completely elucidated, higher frequency $(>30 \mathrm{~Hz})$ oscillations are thought to be involved in the coordination of activity of local neuronal populations (Buzśaki \& Wang, 2012), as well as higher-order cognitive functions (Jensen et al., 2007). Gamma activity has not typically been examined in the context of MDD. However, recent findings of ketamine's antidepressant effects have brought it into the spotlight, and suggest that gamma oscillations may serve as a diagnostic biomarker for MDD and could index treatment response (Fitzgerald \& Watson, 2018). Gamma oscillations are found throughout the brain, and are related to the cortical excitation/inhibition balance, involving both glutamatergic and GABAergic mechanisms. As such, an imbalance in this system has been suggested to underly MDD (Gilbert \& Zarate, 2020).

Increases in resting gamma power following acute ketamine administration have been consistently found in healthy control (de la Salle et al., 2016 [Appendix I]; Forsyth et al., 2018; McMillan et al., 2019; Muthukumaraswamy et al., 2015; Rivolta et al., 2015; Sanacora et al., 2014; Vlisides et al., 2017, 2018) and in MDD samples (Cornwell et al., 2012; Nugent et al., 2019a, 2019b). However, the MDD studies assessed delayed gamma power changes ( 6-9 hours postinfusion), therefore additional acute ketamine administration studies in MDD are required to examine the time course of these increases. Ketamine may increase gamma power by reducing NMDA receptor-mediated input to fast-spiking parvalbumin-expressing GABAergic interneurons and through the disinhibition of excitatory pyramidal neurons (Gilbert \& Zarate, 2020). However, the association with the antidepressant response is likely more complex (Nugent et al., 2019a). 


\subsubsection{Delta and Beta Power}

Also less studied in the context of MDD, elevated delta (1-4Hz; Morgan et al., 2005; Nystrom et al., 1986) and beta (12-30Hz; Grin-Yatsenko et al., 2009; Knott et al., 2001) power have been found in MDD patients as compared to healthy controls, though little evidence has been found regarding their treatment prediction utility. Certain healthy control studies have shown that sub-anesthetic ketamine doses decrease delta (de la Salle et al., 2016 [Appendix I]; Forsyth et al., 2018; Knott et al., 2006; McMillan et al., 2019; Shaw et al., 2015; Vlisides et al., 2017, 2018), and beta (Forsyth et al., 2018; Knott et al., 2006; McMillan et al., 2019; Muthukumaraswamy et al., 2015; Rivolta et al., 2015; Vlisides et al., 2018) power. However, as there is little research involving these bands within a TR MDD population, they may still provide useful information or generate additional hypotheses for future studies.

\subsubsection{Mismatch Negativity (MMN)}

In addition to qEEG activity, ERPs have also been employed in MDD research. These have generally involved the P300, an assessment of novelty and target detection, error-related negativity (ERN) derived from performance-monitoring tasks, visual/emotional tasks producing the late positive potential (LPP) and the vertex positive potential (VPP), or the loudness dependence of the auditory evoked potential (LDAEP), purported to index serotonergic neurotransmission (Bruder et al., 2012). However, for the study of ketamine, a task which can be administered without the need for attention or task performance, and which indexes NMDAR function, was required.

The auditory mismatch negativity (MMN) event-related potential is a commonly studied early electrophysiological measure. It is a frontocentral negative deflection that is observed 120- 
$250 \mathrm{~ms}$ post-stimulus, elicited within paradigms of infrequent (deviant) and repeated (standard) stimuli and quantified by subtracting the standard from the deviant response (Näätänen et al., 2007). The original theory regarding MMN generation suggested that it was the result of auditory "echoic" sensory memory (Näätänen, 1990). A more recent theory has suggested that the MMN reflects a prediction-error based on regularity violation, with the resulting MMN occurring only when a stimulus does not match the prediction based on regularity representations (i.e. the expectation of a standard stimuli following another standard stimuli) encoded from preceding auditory stimuli (Winkler, 2007). The standard stimuli elicits the N1 ERP, also a frontocentral negative deflection observed approximately $90-120 \mathrm{~ms}$ post-stimulus, which represents the encoding of the simple physical features of the auditory stimuli. In a standard oddball paradigm, the repeated administration of the standard stimuli leads to habituation and decreased N1 amplitudes (Näätänen \& Picton, 1987).

Though the MMN is frequently examined within the schizophrenia literature, it is also well-suited for the study of ketamine administration, as it is modulated primarily by NMDA receptor signalling (Rosburg \& Kreitschmann-Andermahr, 2016), and has been suggested as a neurophysiological proxy of target engagement for NMDAR-associated cortical plasticity (Kantrowitz et al., 2018). Additionally, it can be recorded without conscious attention (e.g. during sleep, sedation, and disorders of consciousness; Chennu \& Bekinschtein, 2012). Underlying the scalp-level MMN amplitudes are generators which include the bilateral superior temporal lobe, anterior cingulate gyrus, and right inferior temporal lobe (Waberski et al., 2001). The temporal MMN subcomponent is thought to be generated by pre-perceptual deviance detection (i.e. the processing of simple deviant features), followed by involuntary switching of attention to the auditory deviance by the more frontal regions (i.e. regularity violation of 
complex patterns/unexpected inputs), producing the frontal MMN subcomponent (HofmannShen et al., 2020; Rinne et al., 2000). However, cortical generators have been found to vary according to the paradigm administered, with simpler and more complex stimuli being associated with greater temporal and frontal generator activity, respectively (MacLean et al., 2015).

Certain studies in MDD populations have found reduced (i.e. decreased amplitude, Qiao et al., 2013; Takei et al., 2009) as well as increased (Bissonnette et al., 2020; He et al., 2010; Kähkönen et al., 2007; Restuccia et al., 2016) MMN amplitudes, and longer latencies (Bissonnette et al., 2020; Qiao et al., 2013). A recent study also identified a potential link between MMN amplitude and functional outcomes in MDD patients (Kim et al., 2020). As such, it is possible that reduced amplitudes in MDD may reflect pathological NMDAR functioning, which subsequently leads to impaired functioning.

Spectral decompositions can also be performed on ERP waveforms in order to quantify oscillations underlying amplitude (microvolts $[\mu \mathrm{V}]$ ) and latency (ms). This is accomplished through the conversion from the time domain to the time-frequency domain. Event-related oscillations (ERO) are time-locked to the stimulus and include only measures of evoked (vs. induced) activity, while measures of phase locking (phase locking factor, PLF) are examined via single trial analysis. Specifically, the frontal MMN has been found to be associated with an increase in theta power for deviant stimuli, as well as by theta phase alignment (Fuentemilla et al., 2008). Theta ERO have also been associated with attention and cognitive processes, and may serve an orienting function (Aftanas et al., 2001). The temporal MMN has been found to be associated with theta rhythm phase resetting with no power modulation. Previous studies have found that employing time-frequency analysis, can provide a more sensitive and detailed picture of brain dynamics underlying the MMN (Kaser et al., 2013). 
The association between NMDA antagonism and MMN alterations in amplitude decreases and/or increases in latency have been observed in both human (Rosburg \& Kreitschmann-Andermahr, 2016) and animal studies (Gil-Da-Costa et al., 2013). The focus of many healthy control electrophysiological studies has been on the induction of psychotomimetic effects mimicking positive symptoms of schizophrenia, therefore a bolus dose (ranging from $0.15-30 \mathrm{mg} / \mathrm{kg}$ ) has typically been administered. Oranje et al. (2000) found no reductions in MMN with a comparatively lower dose, however, a recent meta-analysis found that most studies have shown robust decreases in MMN amplitudes using frequency, duration, and intensity deviants, as well as increases in latency, though with a lower effect size (Rosburg \& Kreitschmann-Andermahr, 2016). Studies of the effect of ketamine on the auditory N1 within MMN paradigms have found either no change (de la Salle et al., 2019 [Appendix II]; Heekeren et al., 2008; Kreitschmann-Andermahr et al., 2001) or increased amplitudes (Umbricht et al., 2000). However, this is an important area of investigation, as the lack of change in the N1 with ketamine signals that the reductions observed in MMN amplitude are due primarily to specific neuronal processes which generate the MMN. Finally, ketamine has also been found to reduce auditory evoked theta activity which has been implicated in MMN generation (de la Salle et al., 2019 [Appendix II]; Lee et al., 2018), though additional work is needed.

The administration of the MMN requires no behavioural response or attention (a cognitive domain that is markedly altered with ketamine) as it is capturing pre-attentive detection processes. Combined with its reliance on NMDAR functioning and its purported relation to synaptic plasticity, it could serve as a feasible, non-invasive neurobiomarker for the antidepressant response prediction to glutamatergic agents. 


\subsection{Ketamine-Induced Effects in MDD and their Relationship to Symptom Changes}

Few studies have examined these effects in MDD or related the changes in activity to the antidepressant response (Cao et al., 2019; McMillan et al., 2020; Nugent et al., 2019a). Using a wearable forehead device, treatment responders exhibited increases in low prefrontal alpha, high right prefrontal alpha, decreases in low prefrontal alpha asymmetry, and a decrease in PF theta cordance four hours post-ketamine infusion (Cao et al., 2019). McMillan et al. (2020) observed increases in theta, high beta, low and high gamma power. Beta and gamma power remained elevated throughout the infusion, and theta power returned to baseline four minutes postinfusion initiation. Decreases in delta, alpha, and low beta power were observed, and remained low throughout the infusion. When examining the relationship between change in power and change in antidepressant response, a trend $(\mathrm{p}<.08)$ was observed between increased frontal theta power and antidepressant response (i.e. increased frontal theta power correlated with a larger decrease in MADRS at one-day postinfusion) (McMillan et al., 2020). A MEG study (Nugent et al., 2019a) found that the relationship between ketamine-induced increased gamma power (6-9 hours postinfusion) within multiple brain networks associated with MDD pathophysiology and treatment response were moderated by baseline gamma power levels, i.e. lower baseline $=$ higher post-ketamine gamma power associated with greater decreases in symptoms, and an inverted relationship for higher baseline gamma. These findings indicate that ketamine's mechanism of action may depend on homeostatic dysregulation, and points to the important of inter-individual differences in treatment response.

A MMN paradigm was recently examined in a sample of MDD patients receiving a subanesthetic dose of ketamine $(0.44 \mathrm{mg} / \mathrm{kg})$ in comparison to an active placebo (remifentanil; Sumner et al., 2020). The recordings were carried out approximately 3-4 hours postinfusion, 
when improvements in depressive symptoms are typically observed. The authors found that ketamine significantly increased the $\mathrm{MMN}$ at this time point. As well, a significant relationship between greater forward connectivity modulation between the right primary auditory cortex and right inferior temporal cortex in response to deviant tones with greater decreases in symptoms one day postinfusion was observed, though no relationship with the MMN itself was found. These findings were interpreted as a restoration of sensitivity to prediction errors found in MDD.

\subsection{Objectives and Hypotheses}

The current electrophysiological study was a component of a larger clinical trial (NCT01945047) aimed at enhancing and prolonging the antidepressant effect of ketamine through repeated infusions (Phillips et al., 2019). The objectives were 1) to assess the pharmacodynamic actions of ketamine in patients with TR MDD using resting EEG measures (power, alpha asymmetry, theta cordance, theta ACC-indexed activity) and MMN indices (amplitude, latency, theta ERO, theta PLF, peak MMN generator activity) , 2) to assess how these acute changes, in combination with baseline EEG measures, correlated with acute changes in depressive symptom changes, and 3) to assess the utility of these EEG measures (baseline and early changes) and depressive (baseline and early changes) in predicting initial and prolonged treatment response to repeated ketamine doses. Additionally, EEG measures were examined in relation to decreases in suicidal ideation, as this is an important feature of ketamine's effects and research in this area is lacking.

There have been few studies examining EEG/ERP changes with sub-anesthetic doses of ketamine in TR MDD, and none examining acute electrophysiological changes in relation to early and sustained treatment response. However, we expected to observe similar findings as in previous healthy control studies (non-bolus, continuous sub-anesthetic dose), at least during the 
immediate postinfusion recording indexing acute physiological alterations. It was unknown whether these measures would increase, decrease, or remain unchanged at the two-hour recording time point. Changes with midazolam were expected to be similar to pharmaco-EEG studies of benzodiazepines, including increases in beta, and decreases in alpha power (Breimer et al., 1990).

Similarly, there have been few studies examining EEG/ERP features (baseline and treatment-induced) in the response prediction to ketamine in TR MDD. In prediction studies of response to other therapies for MDD (pharmacotherapy, brain stimulation), no one predictor has been found to be superior, therefore all frequency bands from the resting qEEG recording in addition to MMN-derived measures were examined. Only known baseline EEG predictors and ketamine-induced changes were subsequently related to early and sustained changes in depressive symptoms.

It was expected that those showing greater decreases in depressive symptoms (i.e. responders), would show elevated pre-treatment alpha power and left alpha lateralization, as well as higher pre-treatment theta ACC activity, and would show greater ketamine-induced changes in alpha, theta, and gamma power, theta cordance, theta ACC activity, and MMN indices. Regarding the MMN, an early initial decrease followed by an increase above preinfusion levels in responders could index early increases in synaptic plasticity and/or normalization of prediction error sensitivity deficits. How these acute and two hours postinfusion changes would specifically relate to decreases in immediate vs. sustained depressive symptoms, remained to be explored. Significant predictive power would show support for the use of these non-invasive measures of brain activity as a method of early prediction of response to single and repeated sub-anesthetic doses of ketamine in TR MDD. 
CHAPTER 2: Scalp- and Source-Localized Electrophysiological Correlates and Predictors of the Antidepressant Response to Repeated Ketamine Infusions in Treatment-Resistant Depression

\author{
Sara de la Salle ${ }^{* 1}$, Jennifer L. Phillips ${ }^{2}$, Pierre Blier ${ }^{2}$, Verner Knott ${ }^{1}$ \\ ${ }^{1}$ Clinical Electrophysiology and Cognitive Research Laboratory, University of Ottawa Institute \\ of Mental Health Research, Ottawa, ON, Canada \\ ${ }^{2}$ Mood Disorders Research Unit, University of Ottawa Institute of Mental Health Research, \\ Ottawa, ON, Canada
}

\title{
2.1 Abstract
}

Background: Sub-anaesthetic ketamine doses rapidly reduce depressive symptoms, though additional investigations of the underlying neural mechanisms and the prediction of response outcomes are needed. Electroencephalographic (EEG)-derived measures have shown promise in predicting response to a variety of treatments, and are sensitive to ketamine administration. This study examined their utility in characterizing changes in depressive symptoms with acute and repeated ketamine infusions.

Methods: Recordings were obtained from patients with treatment-resistant major depressive disorder (MDD; $\mathrm{N}=24$ ), enrolled in a multi-phase ketamine trial. During the randomized, doubleblind, crossover phase (Phase 1$)$, patients received ketamine $(0.5 \mathrm{mg} / \mathrm{kg})$ or midazolam (30 $\mu \mathrm{g} / \mathrm{kg}$ ), $\sim 1$ week apart. Three resting, eyes-closed recordings were carried out per session (pre-, post-, $2 \mathrm{hrs}$ postinfusion), and changes in EEG power (delta, theta1/2/total, alpha1/2/total, beta, gamma), alpha asymmetry, theta cordance, and theta source-localized anterior cingulate cortex activity were quantified. The relationships between ketamine-induced changes with early (Phase 1) and sustained (Phases 2,3) decreases in depressive symptoms (Montgomery-Åsberg Depression Rating Score, MADRS) and suicidal ideation (MADRS item 10) were examined. Results: Both substances decreased alpha and theta immediately postinfusion, however, only midazolam increased delta (postinfusion), while only ketamine increased gamma (immediately 
post- and 2 hrs postinfusion). Source-localized ACC activity increased two hours postinfusion. Regional- and frequency-specific ketamine-induced EEG changes were related to and predictive of decreases in depressive symptoms (theta, gamma) and suicidal ideation (alpha). Early and sustained treatment responders differed at baseline in scalp- and source-localized theta.

Conclusions: Ketamine exerts frequency-specific changes on EEG-derived measures, which are related to depressive symptom decreases in treatment-resistant MDD, and provides information regarding early and sustained individual response to ketamine.

Keywords: alpha, antidepressants; cordance; depression; EEG; gamma; ketamine; midazolam; response prediction; theta; treatment-resistance; treatment response. 


\subsection{Introduction}

\subsubsection{Growing Burden of Major Depressive Disorder (MDD)}

Major Depressive Disorder (MDD) is a prevalent (World Health Organization, 2017) and costly (König et al., 2019) illness, with varying individual symptom presentations (Rush, 2007), which can have widespread effects on an individual's daily functioning (Hammer-Helmich et al., 2018) and lifespan (Cuijpers \& Schoevers, 2004). Tragically, it is often comorbid with suicidal ideation (SI, (Nock et al., 2010). MDD is also often highly recurrent (Burcusa \& Iacono, 2007), and while there are many accepted treatments (e.g. pharmacotherapy, psychotherapy, and brain stimulation), their effectiveness is limited (Penn \& Tracy, 2012). Approximately one third of people who seek treatment for MDD do not tolerate or respond to available pharmacotherapy and another third show only a partial response (Kennedy et al., 2001; Rush et al., 2006). These individuals are considered to be "treatment-resistant", i.e. exhibiting a lack of or an inadequate response (Kasper, 2014). There is also a lag time between treatment initiation and therapeutic response for antidepressant medications (Porcelli et al., 2011). Despite the fact that pharmacological effects begin within hours of drug administration, therapeutic effects only begin to manifest within two to four weeks from treatment initiation. This delay in depressive symptom reduction can be critical for the individual due to the possibility of symptom worsening and suicide risk (Ferrari \& Villa, 2017).

\subsubsection{Rising Use of Ketamine as an Antidepressant Agent}

A growing body of research has suggested that abnormalities in the glutamate system may be implicated in the pathophysiology and treatment of depression (Mathews et al., 2012). Ketamine, a dissociative anesthetic, is an N-methyl-D-aspartate receptor (NMDAR) antagonist that has been investigated extensively in rodents and humans within the past two decades (Wei et 
al., 2020). While there are still unknown mechanisms through which ketamine exerts its rapid antidepressant response, the current hypothesis is that it increases glutamate neurotransmission in the prefrontal cortex, which leads to increased synaptic plasticity and neurotrophic signaling through downstream molecular cascades (Feifel, 2016; Lener et al., 2017).

A single sub-anesthetic dose of ketamine has repeatedly been shown to exert rapid antidepressant effects in individuals with unipolar and bipolar depression (Bobo et al., 2016; Caddy et al., 2015). Depressive symptom reductions typically manifest within a few hours, with the effect peaking at 24 hours, and symptoms returning within one week postinfusion (Kishimoto et al., 2016). A recent meta-analysis also found that a single dose of ketamine was able to rapidly reduce SI, and that these effects were partially independent of the antidepressant effect (Wilkinson et al., 2018a). Many early trials used saline as a placebo condition, though a recent comparison with studies utilizing the benzodiazepine midazolam as an "active" placebo (Grunebaum et al., 2018; Murrough et al., 2013a, 2015) suggested that midazolam was superior to saline for blinding integrity (Wilkinson et al., 2019).

While ketamine is effective for the rapid reduction of depressive symptoms, including SI, its limitation lies in its transitory nature. Recent work has focused on specific dosing schedules (twice-weekly, thrice-weekly) aimed at prolonging the antidepressant effects (aan het Rot et al., 2010; Cusin et al., 2017; Murrough et al., 2013b; Shiroma et al., 2014; Singh et al., 2016; Vande Voort et al., 2016; Wilkinson et al., 2018b). Phillips et al. (2019) examined single, repeated (thrice-weekly for 2 weeks), and maintenance (once weekly, for a month) infusions, and found that repeated infusions led to cumulative and sustained reductions in depressive symptoms that were maintained in responders. Importantly, non-responders to single infusions responded to repeated infusions (Phillips et al., 2019). 


\subsubsection{Ketamine-Induced Electrophysiological Changes}

There have been many investigations of the acute electrophysiological effects of subanesthetic doses of ketamine in healthy human participants (McMillan \& Muthukumaraswamy, 2020), with consistent findings of decreases in electroencephalography (EEG)-derived alpha (8.5-12Hz; de la Salle et al., 2016 [Appendix I]; Forsyth et al., 2018; Kochs et al., 1996; McMillan et al., 2019; Muthukumaraswamy et al., 2015; Vlisides et al., 2017, 2018) and increases in gamma $(30-50 \mathrm{~Hz})$ power (de la Salle et al., 2016 [Appendix I]; Forsyth et al., 2018; McMillan et al., 2019; Muthukumaraswamy et al., 2015; Rivolta et al., 2015; Sanacora et al., 2014; Vlisides et al., 2017, 2018). Alpha activity is inversely related to the brain's state of activation and arousal, and has also been suggested as an index of relative cortical inhibition/deactivation (Klimesch, 2012; Klimesch et al., 2007; Neuper \& Pfurtscheller, 2001), while resting gamma activity is related to the cortical excitation (glutamatergic)/inhibition (GABAergic) balance (Gilbert \& Zarate, 2020).

While the data is less consistent with the lower frequency bands, certain studies have shown that sub-anesthetic doses decrease delta (1-4Hz) power (de la Salle et al., 2016 [Appendix I]; Forsyth et al., 2018; Knott et al., 2006; McMillan et al., 2019; Shaw et al., 2015; Vlisides et al., 2017, 2018), while increases in frontal theta $(4-8 \mathrm{~Hz})$ have been found in studies that employed a bolus dose (Forsyth et al., 2018; McMillan et al., 2019; Muthukumaraswamy et al., 2015). Studies with a slow sub-anesthetic infusion method only, however, have found decreases in theta power (de la Salle et al., 2016 [Appendix I]; Knott et al., 2006; Vlisides et al., 2017, 2018). Prefrontal theta cordance, a combination of absolute and relative power and a proposed measure of cerebral perfusion, has been found to decrease following ketamine infusion in healthy controls (Horacek et al., 2010; Sanacora et al., 2014). Horacek et al. (2010) proposed that acute 
ketamine-induced neurophysiological changes were similar to the gradual monoaminergic-based antidepressant changes, and that this change could serve as a marker and a predictor of the rapid antidepressant effect. Changes in beta power have been less clear, but have generally shown decreases (Forsyth et al., 2018; Knott et al., 2006; McMillan et al., 2019; Muthukumaraswamy et al., 2015; Rivolta et al., 2015; Vlisides et al., 2018).

Only a handful of studies have examined these effects in MDD samples, as well as the relationship between changes in power and the antidepressant response (Cao et al., 2019; McMillan et al., 2020; Nugent et al., 2019a). Using a wearable forehead device, treatment responders exhibited increases in low prefrontal alpha, high right prefrontal alpha, decreases in low prefrontal alpha asymmetry, and a decrease in PF theta cordance four hours post-ketamine infusion (Cao et al., 2019). In another study, gamma power assessed via magnetoencephalography (MEG) was found to increase $\sim 6-9$ hours postinfusion (Nugent et al., 2019a). The antidepressant response was moderated by baseline gamma power, in that higher postketamine power was related to greater decreases in depressive symptoms in individuals with lower baseline gamma; the opposite pattern was found for those with higher baseline gamma. Finally, McMillan et al. (2020), examining frequency-specific changes over the course of a 16min recording, found increases in theta, high beta, low and high gamma power, with beta and gamma power remaining elevated throughout the infusion, and theta power returning to baseline 4mins postinfusion initiation. Decreases in delta, alpha, and low beta power were observed, and remained low throughout the infusion. When examining the relationship between change in power and change in antidepressant response, a trend $(\mathrm{p}<.08)$ existed between increased frontal theta power and antidepressant response (i.e. increased frontal theta power correlated with a 
larger decrease in Montgomery-Åsberg Depression Rating Score [MADRS] at one-day postinfusion) (McMillan et al., 2020).

\subsubsection{Prospective EEG Predictors of Ketamine's Antidepressant Effects}

The potential for early (i.e. before treatment [baseline] or early within treatment [one-two weeks]) identification of treatment responders is crucial; despite the fact that ketamine's antidepressant effects are rapid, certain patients may not respond until multiple repeated infusions, or may not respond at all. As with most treatments for MDD, it is difficult to predict who will benefit from a given treatment. However, baseline and early changes in electrophysiological measures have shown promise in predicting antidepressant treatment response to a variety of therapies (Iosifescu, 2011; Lai, 2019; Olbrich \& Arns, 2013); though more work is required in order to improve its clinical reliability, due, in part, to under-powered studies and depression heterogeneity (Widge et al., 2019). Nevertheless, EEG is a strong candidate for response prediction as it is non-invasive, widely available, has a relatively low cost, and provides continuous millisecond-by-millisecond information regarding cortical electrical activity (Olbrich \& Arns, 2013). The simplest and most versatile method is eyes-closed resting EEG activity, which allows for the offline quantification and analysis of slow (delta, theta) and fast (alpha, beta, gamma) frequencies in terms of power $\left(\mu \mathrm{V}^{2}\right)$ and source-localized current source density $\left(\mathrm{A} / \mathrm{m}^{2}\right)$. The most predictive of the proposed measures are the theta and alpha bands (Schiller, 2019).

Elevated pre-treatment alpha power, most consistently in the posterior regions, has been shown to differentiate responders and non-responders to TCAs and SSRIs (Bruder et al., 2001). Pre-treatment left alpha lateralization has also shown predictive ability to treatment response with TCAs and SSRIs (Bruder et al., 2001, 2008). Frontal theta band power, presumably 
reflecting altered activity in the anterior cingulate cortex (ACC) and with implications for affective processing (Jaworska et al., 2012), have indicated both reduced and increased pretreatment theta in responders, as well as reduced relative theta power at 1 week post-treatment (Iosifescu, 2011). However, the use of EEG source-localization methods have consistently shown higher pre-treatment theta ACC activity to be predictive of antidepressant treatment response (Pizzagalli, 2011; Pizzagalli et al., 2018). Another predictive measure, early decreased cordance (after 1 week of treatment) derived from prefrontal (PF) and/or midline and right frontal (MRF) regions, has been found to predict treatment response, to multiple treatment modalities (Bares et al., 2007, 2008; Broadway et al., 2012; Hunter et al., 2018). The predictive ability of delta and beta power for the purposes of antidepressant treatment response have not been extensively examined.

\subsubsection{The Current Study}

This electrophysiological study was a component of a larger clinical trial (NCT01945047) aimed at enhancing and prolonging the antidepressant effect of ketamine through repeated infusions (Phillips et al., 2019). The primary objectives were: 1) to assess the electrophysiological actions of ketamine in TR MDD using resting EEG measures (power, alpha asymmetry, theta cordance, theta ACC-indexed activity), 2) to assess how acute changes in these measures, in combination with baseline features, correlate with changes in depressive/SI symptom changes, and 3) to assess the utility of these EEG measures (baseline and early changes) and depressive/SI symptoms (baseline and early changes) in predicting initial and prolonged treatment response to repeated ketamine doses.

Consistent with previous work, we expected that alpha power would decrease and gamma would increase with acute ketamine administration. Further, we expected that baseline 
electrophysiological measures (power, alpha asymmetry, theta cordance, ACC activity) and acute changes in these measures, in combination with baseline and acute changes in depressive symptoms, would be useful in characterizing and predicting the early and sustained antidepressant response to ketamine. As there has been few studies examining these response predictors with ketamine in patients with TR MDD, we had no specific hypotheses regarding which predictors (or their combination) would be most salient.

\subsection{Methodology}

\subsubsection{Participants}

24 outpatient participants (males and females, aged 18-65) with treatment-resistant depression who were enrolled in larger single-center randomized controlled trial (Phillips et al., 2019) participated in this add-on electrophysiological arm. Participant recruitment was from physician referrals and media advertisements. Assessments were conducted in the Mood Disorders Research Unit of the Royal's Institute of Mental Health Research in Ottawa, Canada.

For inclusion, patients attended a screening session and were required to (i) have a primary Axis I diagnosis of MDD, single or recurrent, and without psychotic features (confirmed using the Mini-International Neuropsychiatric Interview, Appendix III, Sheehan, 1998), as assessed using criteria from the Diagnostic and Statistical Manual of Mental Disorders, 4th edition (DSM-IV-TR) (American Psychiatric Association, 2000), and (ii) have treatmentresistant MDD, defined as a failure to respond adequately to at least two antidepressant medication trials (of different pharmacological classes) and two augmentation strategies for a minimum of six weeks during the present depressive episode (defined using the Antidepressant Treatment History Form, Appendix IV, Sackeim, 2001). Participants were permitted to maintain stable dosages (i.e. $>6$ weeks) of concomitant psychotropic medications, with no changes 
throughout the trial. A MADRS (Appendix V, Montgomery \& Asberg, 1979) score of $\geq 25$ was required at screening and randomization, with no more than $20 \%$ improvement between visits. Exclusion criteria were: (i) current or past substance abuse or dependence (DSM-IV-TR-defined or positive urine screen), (ii) psychotic symptoms, (iii) a history of mania or hypomania, (iv) a body mass index of $\geq 35$, and (v) any other unstable medical conditions identified during the physical examination (i.e. vital signs, weight, electrocardiogram, blood tests, and urinalysis); pregnancy was also exclusionary.

All participants provided informed consent for both the clinical trial and add-on EEG arm. The study was approved by the Research Ethics Boards of the Royal Ottawa Health Care Group. This study was conducted in accordance with the Tri-Council Policy Statement on Ethical Conduct for Research Involving Humans.

\subsubsection{Study Design}

The clinical study involved three distinct phases. Phase 1 followed a randomized, doubleblind cross-over design, with participants randomly assigned to receive either ketamine or an active placebo (midazolam, Wilkinson et al., 2019). The participants and the study staff in immediate contact with the participants were blind to the treatment. Participants were required to return to $80 \%$ of their baseline MADRS scores in order to receive the second infusion ( $>7$ days between sessions). The same criteria were used to determine their progression to Phase 2. During each of the two sessions, EEG recordings were performed before, immediately following, and two hours postinfusion (Figure 2.1). 
a.

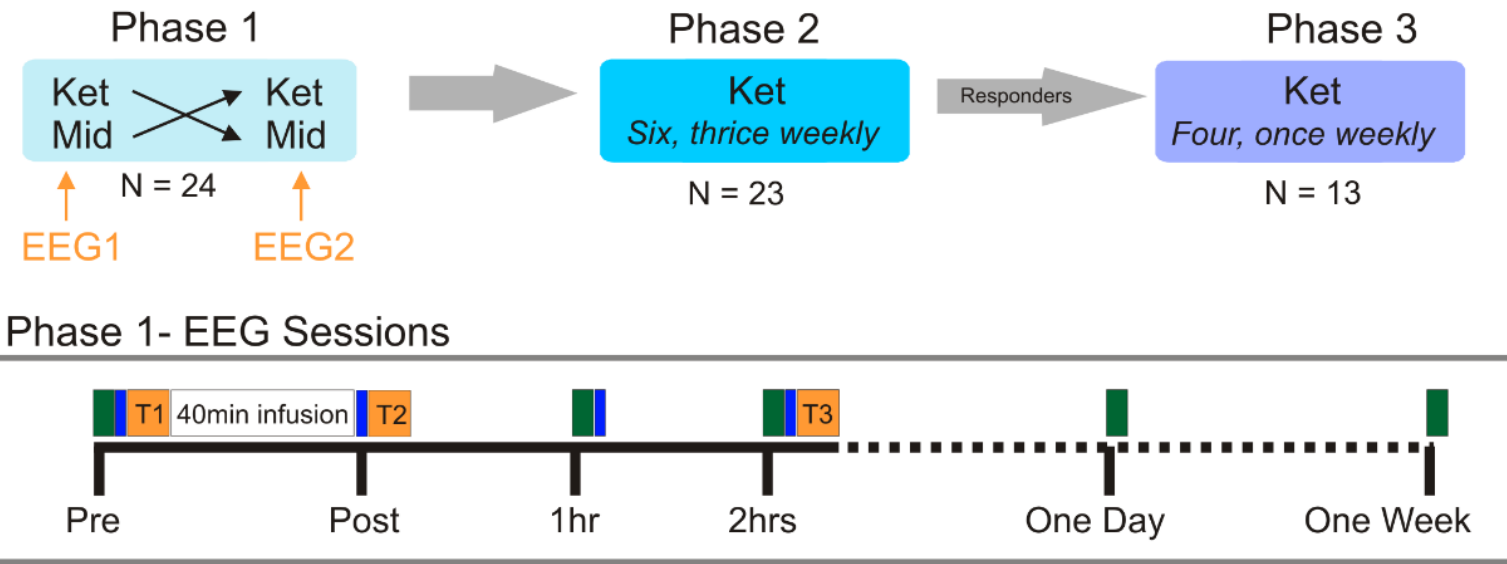

MADRS

CADSS/BPRS

EEG Recording

Figure 2.1. Timeline of three study phases (a) and timeline of EEG recording sessions of Phase 1 (b).

All participants entered Phase 2, which involved the acute repeated administration of 6 ketamine infusions (open-label), thrice weekly over a period of 2 weeks. Only responders (i.e. $\geq$ $50 \%$ decrease in MADRS total score from baseline [prior to first infusion in Phase 1] to the end of Phase 2) were enrolled in Phase 3, the maintenance phase, which involved the administration of 4 ketamine infusions (open-label), once weekly over a period of 4 weeks.

\subsubsection{Drug Administrations}

Ketamine hydrochloride (Ketalar®, ERFA Canada Inc., Montreal, QC; $0.5 \mathrm{mg} / \mathrm{kg}$, diluted in $0.9 \%$ saline) was given throughout the three phases, and midazolam $(30 \mu \mathrm{g} / \mathrm{kg}$ diluted in saline) was administered once during Phase 1. Both were administered over 40 minutes by IV pump by a study physician and research nurse within an outpatient setting. Vitals (blood pressure, pulse, oxygen saturation) were monitored throughout the infusion and postinfusion to ensure a return to preinfusion levels. Participants were also required to abstain from 
benzodiazepines (from the preceding day (Frye et al., 2015) and grapefruit juice (day of infusion (Peltoniemi et al., 2012).

\subsubsection{EEG Acquisition}

The participant was asked to sit still with their eyes closed for three minutes. EEG activity was acquired according to standard pharmaco-EEG procedures (Jobert et al., 2012). The montage included $32 \mathrm{Ag}^{+} / \mathrm{Ag}^{+} \mathrm{Cl}^{-}$passive electrodes (placed according to the $10-20$ international EEG system; Figure 2.2); an electrode placed on the nose served as a reference and a midforehead electrode (AFz) served as the ground. Additional electrodes were placed on the supraand sub-orbital ridges of the right eye and on the external canthus of both eyes to record vertical (VEOG) and horizontal (HEOG) electro-oculographic activity. Recordings were performed using a Brain Vision ${ }^{\circledR}$ Quickamp amplifier and Brain Vision Recorder ${ }^{\circledR}$ (Brain Products, Germany), with amplifier bandpass filters and sampling rate set to $0.1-100 \mathrm{~Hz}$ and $500 \mathrm{~Hz}$, respectively. Electrode impedances were maintained $<5 \mathrm{k} \Omega$.

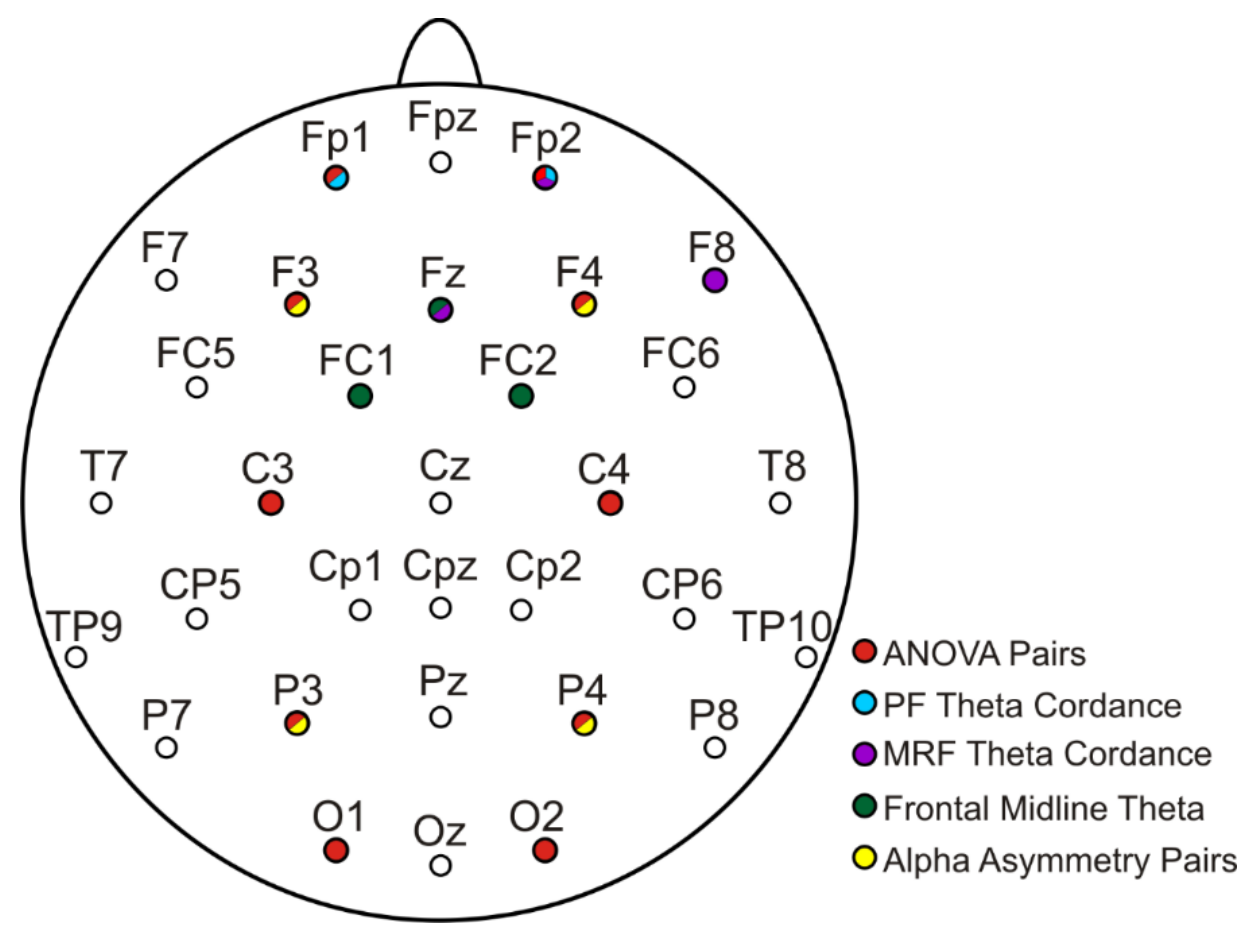

Figure 2.2. Electrode recording montage and electrode groupings for analysis 


\subsubsection{EEG Analysis}

\subsubsection{Scalp Surface-Level Power and Derived Measures}

Off-line processing was performed using Brain Vision ${ }^{\circledR}$ Analyzer Version 2.1 software (Brain Products, Munich, Germany). First, raw data was visually inspected for prominent ocular/muscle/cardiac contamination; any contaminated segments were removed. Data was then re-referenced to linked mastoids, bandpass filtered from $0.1-70 \mathrm{~Hz}(24 \mathrm{~dB} /$ oct; $60 \mathrm{~Hz}$ notch filter), ocular-corrected (Gratton et al., 1983), segmented (2.048ms) and inspected for artifacts (voltages $\pm 75 \mu \mathrm{V}$, faulty channels, drift). The resulting corrected, non-overlapping epochs were subjected to a Fast Fourier Transform algorithm (Hanning window with $10 \%$ cosine taper) for computation of absolute $\left(\mu \mathrm{V}^{2}\right)$ spectral power of delta $(1-4 \mathrm{~Hz})$, theta1 $(4-6 \mathrm{~Hz})$, theta2 $(6-8 \mathrm{~Hz})$, theta total (4-8 Hz), alpha1 $(8.5-10.5 \mathrm{~Hz})$, alpha2 $(10.5-12.5 \mathrm{~Hz})$, alpha total $(8.5-12.5 \mathrm{~Hz})$, beta $(12.5-30$ $\mathrm{Hz}$ ), and gamma (30-50 Hz). Values were then ln-transformed (Gasser et al., 1982) for statistical analyses. Alpha (alpha1, alpha2, and alphaT) power asymmetry was calculated from lntransformed data from frontal electrodes $(\mathrm{F} 4-\mathrm{F} 3 / \mathrm{F} 4+\mathrm{F} 3)$ and posterior electrodes $(\mathrm{P} 4-\mathrm{P} 3 / \mathrm{P} 4+\mathrm{P} 3$; Arns et al., 2016). A positive score indicates greater left hemisphere activation (higher left alpha). Theta cordance values were computed via a custom Matlab script of the cordance algorithm provided by the developers, as defined in Leuchter et al. (1999). This algorithm involves the computation of a re-attributed montage (30 pairs, 19 electrodes) in which absolute and relative power are calculated for each bipolar pair of neighbouring electrodes, followed by a square-root and a z-transformation. The normalized absolute and relative values are then summed for each electrode and for each frequency band. Average cordance values from the two prefrontal electrodes (Fp1, Fp2) as well as the MRF region (Fz, Fp2, F4, F8) were calculated for the theta band only. 


\subsubsection{Theta Source-Localized ACC Activity}

In order to derive ACC theta activity, EEG data was referenced to the average (Jaworska et al., 2012) and computed using exact low-resolution electromagnetic tomography software (eLORETA; version 2081104; 89). eLORETA is a weighted minimum non-linear inverse solution method applied to EEG recordings for computation of three-dimensional distribution of electric cortical activity with zero location error (Pascual-Marqui et al., 2011). Current density $\left(\mathrm{A} / \mathrm{m}^{2}\right)$ was estimated as at three regions of interest: rostral ACC (BA24, 32 voxels), dorsal ACC (BA32, 91 voxels) and the subgenual ACC (BA25, 12 voxels); ROIs are displayed in Figure 2.3. These regions were defined based on the Montreal Neurologic Institute average magnetic resonance imaging (MRI) brain (MNI 152) (Mazziotta et al., 2001) consisting of 6239 voxels (5 $\times 5 \times 5 \mathrm{~mm}^{3} /$ voxel) and restricted to cortical gray matter/hippocampus. This method has been cross-validated with functional and structural MRI, positron emission tomography (PET) and intracranial recordings (Mulert et al., 2004; Pizzagalli et al., 2004; Seeck et al., 1998; Vitacco et al., 2002; Worrell et al., 2000).

\subsubsection{Clinical Outcomes and Dissociative Symptom Assessment}

Clinical outcome measures for the primary study and specific outcomes related to SI are presented in Philips et al. (2019) and Philips et al. (2020), respectively. For this smaller sample of patients who completed the EEG component of Phase 1, mean ( \pm S.D.) MADRS total scores and item 10 (to assess SI) are presented. MADRS item 10 has been found to be a reliable assessment of rapid decrease in SI with ketamine (Ballard et al., 2015). Baseline measures were derived from the first session (regardless of drug). Comorbid disorders were assessed using the MINI at screening. Responders were patients who demonstrated $\geq 50 \%$ decrease in MADRS total score. During Phase 1, the Brief Psychiatric Rating Scale-Positive Symptoms subscale (BPRS-P; 
Appendix VI; Grunebaum et al., 2018; Overall \& Gorham, 1962), which assesses conceptual disorganization, mannerisms and posturing, grandiosity, hostility, suspiciousness, hallucinatory behaviour, unusual thought content, and excitement, was administered at preinfusion, immediately postinfusion, 1 and $2 \mathrm{hrs}$ postinfusion.
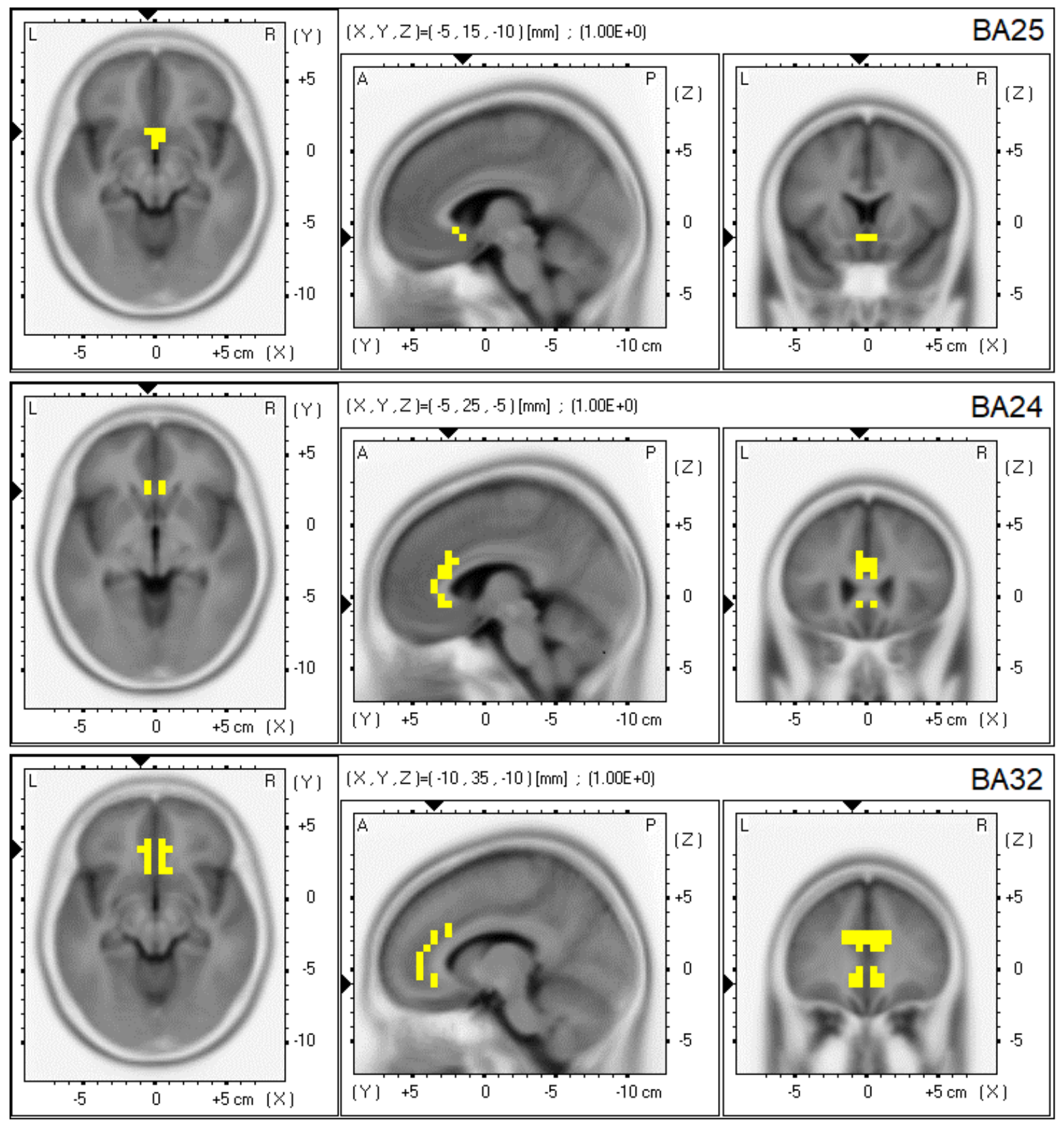

Figure 2.3. Anterior cingulate cortex (ACC) regions of interest (ROIs): $\operatorname{sg} A C C$ (BA25), top; rACC (BA24), middle; dACC (BA32), bottom. 


\subsubsection{Statistical Analysis}

Statistical analyses were carried out with the Statistical Package for Social Sciences (IBM, 2016). To assess the acute regional and frequency specific changes, power values for each frequency band were analyzed with separate repeated measures analysis of variance (ANOVA) involving drug condition (KET, ketamine; MID, midazolam), time (preinfusion, immediately postinfusion, 2 hours postinfusion), region (prefrontal [Fp1, Fp2], frontal [F3, F4], central [C3, $\mathrm{C} 4]$, posterior [P3, P4], occipital [O1, O2]) and laterality (left, right) factors. The main effects and interactions of interest were drug condition and time. A priori planned comparisons for drug condition $\mathrm{x}$ time interactions are reported, unless there was significant 'drug condition $\mathrm{x}$ time $\mathrm{x}$ region' or 'drug condition $\mathrm{x}$ time $\mathrm{x}$ hemisphere' interaction. Similar ANOVAs (with drug condition and time) were carried out for alpha asymmetry (pair: F4-F3, P4-P3), theta cordance (separately for PF, MRF), frontal midline theta power, and source-localized ACC power (BA). Significant $(\mathrm{p}<0.05)$ Greenhouse-Geisser estimates were followed up with Bonferroni adjusted pairwise comparisons. Partial eta-squared $\left(\eta p^{2}\right)$ effect sizes are reported.

Relationships between baseline electrophysiological measures known to be related to treatment outcome (theta, alpha power, alpha asymmetry) as well as absolute change in electrophysiological measures (i.e. postinfusion - preinfusion, $\Delta$ postinfusion and $2 \mathrm{hrs}$ postinfusion - preinfusion, $\Delta 2 \mathrm{hrs}$ postinfusion) were examined in relation to absolute change in MADRS and MADRS SI assessments (early change scores [i.e. 2hrs postinfusion - preinfusion, ' $\Delta 2$ hrs'; one day - preinfusion, ' $\Delta 1 \mathrm{~d}$ '), sustained change scores [i.e. Post-Phase 2 - Baseline, ' $\Delta \mathrm{Ph} 2$ '; Post-Phase 3 - Baseline, ' $\Delta \mathrm{Ph} 3$ ']) using Pearson correlations. Only variables that exhibited ketamine-induced changes were examined. Correlations were also carried out for 
change in BPRS-P symptoms ( $\triangle \mathrm{BPRS}-\mathrm{P})$ with alpha power, as this band has been found to be related to perceptual/dissociative symptoms with ketamine (de la Salle et al., 2016 [Appendix I]).

Only electrophysiological variables (baseline and ketamine-induced changes) that were correlated with early and sustained change in MADRS were examined in terms of their predictive ability. Separate stepwise multiple regressions (Criteria: Probability-of-F-to-enter $<=$ .05 , Probability-of-F-to-remove $>=.10$ ) were performed with $\Delta 2 \mathrm{hrs}, \Delta 1 \mathrm{~d}, \Delta \mathrm{Ph} 2$, and $\Delta \mathrm{Ph} 3$ for MADRS total score and MADRS SI as dependent variables. As baseline and early change in depressive symptoms can be predictive of later response, we included baseline MADRS scores for all models, and early change in MADRS in the regressions for later response times (i.e. $\Delta 2 \mathrm{hrs}$ within the model predicting $\Delta 1 \mathrm{~d}$, and $\Delta 2 \mathrm{hrs}$ and $\Delta 1 \mathrm{~d}$ within models predicting $\Delta \mathrm{Ph} 2, \Delta \mathrm{Ph} 3)$. As an exploratory measure, baseline variables were compared with Mann-Whitney U-tests in responders and non-responders at $1 \mathrm{~d}$ and end of $\mathrm{Ph} 2$.

To explore the previous finding of baseline influence on change in gamma power and depressive symptoms, separate linear fixed effects models with immediately post- and $2 \mathrm{hrs-}$ postinfusion gamma power as the dependent variable, $\Delta 2 \mathrm{hrs}$ MADRS and $\triangle 1 \mathrm{~d}$ MADRS as main effects, and baseline gamma power as a covariate, were conducted.

\subsection{Results}

\subsubsection{Demographics, clinical outcome, and dissociative symptom measures}

The outcomes of the primary trial sample are presented in Philips et al. (2019). 24 patients were included in the EEG portion of the study, and 23 completed both EEG sessions of Phase 1. One patient dropped out during Phase 2 after three infusions. None of the patients had mania, hypomania, post-traumatic stress disorder, alcohol or substance abuse or dependence, 
psychotic features, anorexia nervosa, bulimia nervosa, or antisocial personality disorder.

Demographic features and clinical characteristics of the participants are listed in Table 2.1.

Table 2.1 Demographic features and clinical characteristics of participants $(\mathrm{N}=24)$. Means and standard deviations are presented.

\begin{tabular}{|l|l|}
\hline Variable & Means $( \pm$ S.D. $)$, Ns \\
\hline Sex & $14 \mathrm{~F} / 10 \mathrm{M}$ \\
\hline Age (years) & $41.7 \pm 12.3$ \\
\hline Weight & $81.1(17.7)$ \\
\hline Body mass index & $27.1(4.8)$ \\
\hline Length of current episodes (years) & $5.7(4.4)$ \\
\hline Major Depressive Episodes, Single/Recurrent & $12 / 12$ \\
\hline Failed Antidepressant Trials ${ }^{\mathrm{a}}$, Mean (SD) & $3.1(1.5)$ \\
\hline Failed Antidepressant Augmentations ${ }^{\mathrm{a}}$, Mean $(\mathrm{SD})$ & $2.8(1.0)$ \\
\hline ECT Nonresponder in Current Episode, $n(\%)$ & $5,20.8 \%$ \\
\hline rTMS Nonresponder in Current Episode, $n(\%)$ & $1,4.2 \%$ \\
\hline Lifetime History of Suicide Attempt, $n(\%)$ & $6,25.0 \%$ \\
\hline Comorbid Panic Disorder ${ }^{\mathrm{b}}, n \%$ & $2,8.3 \%$ \\
\hline Comorbid Agoraphobia ${ }^{\mathrm{b}}, n \%$ & $6,25.0 \%$ \\
\hline Comorbid Social Phobia ${ }^{\mathrm{b}}, n \%$ & $6,25.0 \%$ \\
\hline Comorbid Obsessive Compulsive Disorder $^{\mathrm{b}}, n \%$ & $2,8.3 \%$ \\
\hline Comorbid Generalized Anxiety Disorder,$n \% \%$ & $5,20.8 \%$ \\
\hline
\end{tabular}

ECT, Electroconvulsive therapy; F, female; M, male; MADRS, Montgomery-Åsberg Depression Rating Scale (Montgomery \& Asberg, 1979); rTMS, repetitive Transcranial Magnetic Stimulation.

${ }^{a}$ Number of failed antidepressant trials and augmentations during current episode according to the Antidepressant Treatment History Form (Sackeim, 2001)

${ }^{\mathrm{b}}$ Assessed with the Mini-International Neuropsychiatric Interview (Sheehan, 1998)

The response rate for ketamine at 2 hours was $16.7 \%$ and $25 \%$ at one day. The average number of days between the two sessions of Phase 1 was 9.7 ( \pm 4.6 S.D., range 7-22 days). The response rate at the end of Phase 2 was 56.5\%. 13 patients were entered into Phase 3, and the response rate was $76.9 \%$ (Table 2.2). The remission rate at the end of Phase 2 was $21.7 \%$ (5 responders, 18 non-responders) was 53.8\% at the end of Phase 3 (7 responders, 6 nonresponders). 
Table 2.2 a. Montgomery-Åsberg Depression Rating Scale (MADRS) means \pm S.D.; b. Changes in Brief Psychiatric Rating Scale - Positive Symptoms (BPRS-P), means \pm S.D.

\begin{tabular}{|c|c|c|c|c|c|c|c|c|}
\hline a. & & Baseline & $\begin{array}{c}\text { Pre } \\
\text { infusion }\end{array}$ & $\begin{array}{l}2 \text { Hours } \\
\text { Post }\end{array}$ & $\begin{array}{c}\text { One Day } \\
\text { Post }\end{array}$ & $\begin{array}{c}\text { One Week } \\
\text { Post }\end{array}$ & $\begin{array}{c}\text { End of } \\
\text { Phase } 2\end{array}$ & $\begin{array}{c}\text { End of } \\
\text { Phase } 3\end{array}$ \\
\hline \multirow{2}{*}{$\begin{array}{l}\text { MADRS } \\
\text { Total } \\
\text { Score }\end{array}$} & KET & \multirow{2}{*}{$\begin{array}{l}34.7 \\
(4.1)\end{array}$} & $34.3(4.5)$ & $25.1(8.5)$ & $23.1(8.8)$ & $28.7(9.3)$ & \multirow{2}{*}{$\begin{array}{c}20.4 \\
(13.1)\end{array}$} & \multirow{2}{*}{$\begin{array}{l}11.2 \\
(6.7)\end{array}$} \\
\hline & MID & & $34.1(5.3)$ & $30.5(6.4)$ & $31.0(5.3)$ & $33.0(6.6)$ & & \\
\hline \multirow{2}{*}{$\begin{array}{l}\text { MADRS } \\
\text { SI }\end{array}$} & KET & \multirow{2}{*}{$\begin{array}{l}2.96 \\
(1.4)\end{array}$} & $2.75(1.6)$ & $1.2(1.5)$ & $1.2(1.5)$ & $1.8(1.8)$ & \multirow{2}{*}{$\begin{array}{c}1.1 \\
(1.6)\end{array}$} & \multirow{2}{*}{$0.4(0.9)$} \\
\hline & MID & & $2.6(1.6)$ & $2.2(1.7)$ & $1.8(1.7)$ & $2.5(1.7)$ & & \\
\hline \multirow{2}{*}{ Response } & KET & - & - & $\begin{array}{c}16.7 \% \\
(4 \mathrm{R} / 20 \mathrm{NR})\end{array}$ & $\begin{array}{c}25.0 \% \\
(6 \mathrm{R} / 18 \mathrm{NR})\end{array}$ & $\begin{array}{c}8.3 \% \\
(2 \mathrm{R} / 22 \mathrm{NR})\end{array}$ & \multirow{2}{*}{$\begin{array}{c}56.5 \% \\
(13 \mathrm{R} / \\
10 \mathrm{NR})\end{array}$} & \multirow{2}{*}{$\begin{array}{l}76.9 \% \\
(10 \mathrm{R} / \\
3 \mathrm{NR})\end{array}$} \\
\hline & MID & & & $\begin{array}{c}0.0 \% \\
(24 \mathrm{NR}) \\
\end{array}$ & $\begin{array}{c}0.0 \% \\
(23 \mathrm{NR}) \\
\end{array}$ & $\begin{array}{c}0.0 \% \\
(23 \mathrm{NR}) \\
\end{array}$ & & \\
\hline
\end{tabular}

\begin{tabular}{|l|l|c|c|c|c|}
\hline b. & & Preinfusion & Postinfusion & 1hr postinfusion & 2hrs postinfusion \\
\hline BPRS-P & KET & $8.2(0.4)$ & $10.7(3.5)$ & $8.0(0.0)$ & $8.0(0.0)$ \\
\cline { 2 - 6 } Score & MID & $8.0(0.0)$ & $8.0(0.2)$ & $8.0(0.0)$ & $8.0(0.2)$ \\
\hline
\end{tabular}

BPRS-P, Brief Psychiatric Rating Scale - Positive Symptoms; KET, ketamine; MADRS, Montgomery-Åsberg Depression Rating Scale (Montgomery \& Asberg, 1979); MID, midazolam; NR, non-responder; R, responder; SI, suicidal ideation

\subsubsection{Regional- and Frequency-specific Changes with Ketamine and Midazolam}

\subsubsection{Delta}

rmANOVAs indicated main effects of drug condition $\left(\mathrm{F}[1,22]=7.09, p=.01, \eta \mathrm{p}^{2}=.24\right.$;

midazolam $>$ ketamine $[\mathrm{p}=.01])$, but not time $\left(\mathrm{F}[2,44]=1.94, p=.16, \eta \mathrm{p}^{2}=.08\right)$. Within the drug $\mathrm{x}$ time $\left(\mathrm{F}[2,44]=2.03, p=.14, \eta \mathrm{p}^{2}=.09\right)$ interaction, delta power was increased from preto postinfusion $(p=.04)$. No changes were observed in the ketamine condition. At postinfusion, there was a significant difference between drug conditions (MID $>$ KET, $p=.009$ ). Average delta power and topographical headmaps at each time point and drug condition are displayed in

\section{Figure 2.4.}



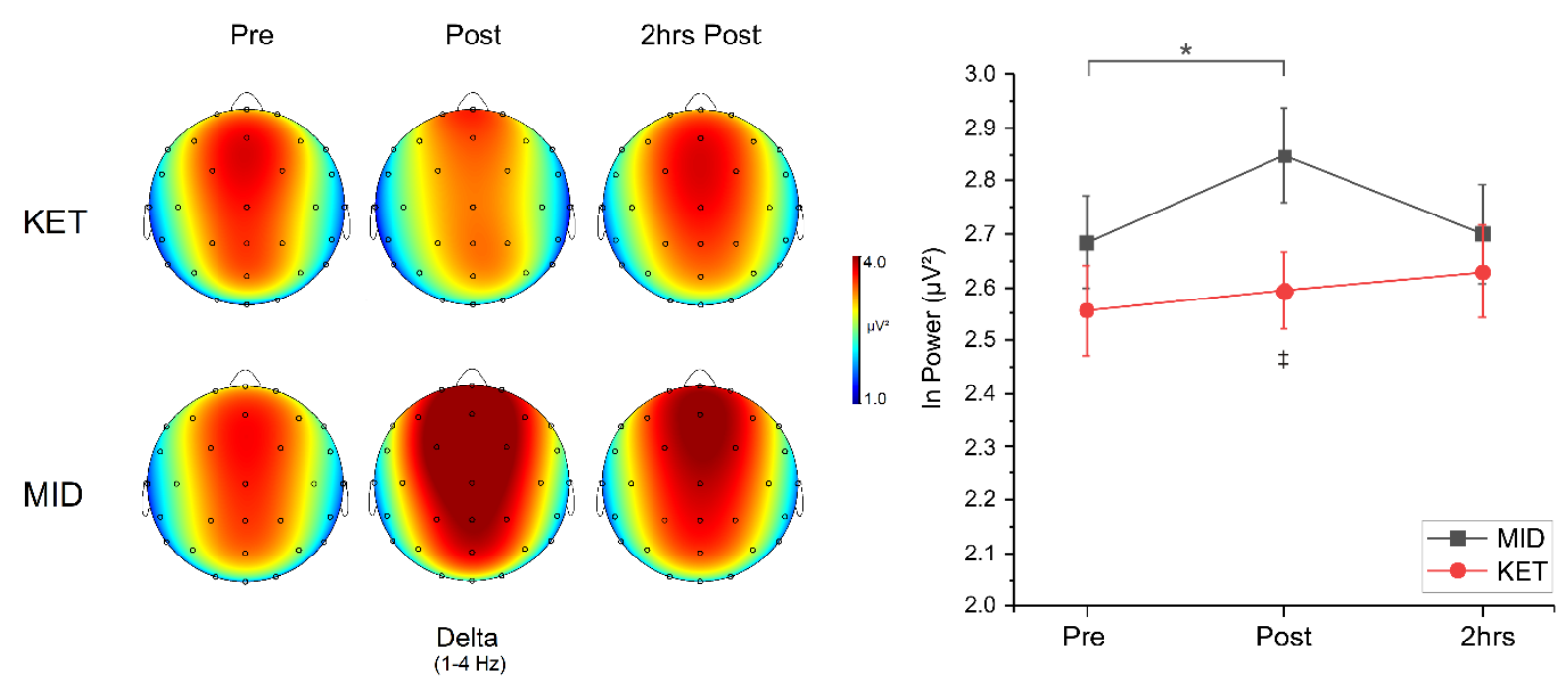

Figure 2.4. Average delta power $\left(\mu \mathrm{V}^{2}\right) \pm$ S.E. and topographical headmaps at each time point and drug condition. $*=$ time comparison, $\$=$ drug condition comparison, $\mathrm{p}<.05$

\subsubsection{Theta}

\subsection{Theta1}

Main effects of drug condition $\left(\mathrm{F}[1,22]=8.50, p=.008, \eta \mathrm{p}^{2}=.29\right)$ and time $(\mathrm{F}[2,44]=$ $\left.5.69, p=.006, \eta p^{2}=.21\right)$ were found for theta1. Power in the midazolam session was greater than with ketamine $(p=.008)$; thetal power postinfusion was reduced as compared to pre- $(p=$ $.02)$ and $2 \mathrm{hrs}$ postinfusion $(p=0.04)$. This finding was mirrored in the planned drug $\mathrm{x}$ time $\left(\mathrm{F}[2,44]=2.34, p=.11, \eta \mathrm{p}^{2}=.01\right)$ interaction, but only for ketamine (postinfusion $<$ preinfusion $[p=.02]$ and $<2 \mathrm{hrs}$ post infusion $[p=.02])$, as well as midazolam being greater than ketamine (i.e. reducing theta1 power to a greater degree) at postinfusion $(p=.003)$.

\subsection{Theta2}

Main effects of drug condition $\left(\mathrm{F}[1,22]=4.59, p=.04, \eta \mathrm{p}^{2}=.17\right)$, time $(\mathrm{F}[2,44]=12.79$, $\left.p=.0001, \eta p^{2}=.39\right)$, and a drug $\mathrm{x}$ time $\mathrm{x}$ region $\left(\mathrm{F}[2.5,54.9]=3.13, p=.04, \eta p^{2}=.12\right)$ interaction were found for theta2. Theta2 power in the midazolam session was greater than with 
ketamine $(p=.008)$; power postinfusion was reduced as compared to pre- $(\mathrm{p}=.0001)$ and $2 \mathrm{hrs}$ postinfusion $(p=0.004)$. Within a planned drug $\mathrm{x}$ time $\left(\mathrm{F}[2,44]=1.72, p=.19, \eta \mathrm{p}^{2}=.07\right)$ interaction, power was reduced from pre- to postinfusion $(p=.02)$ for midazolam, while for ketamine power was reduced at postinfusion $(p=.002)$ as compared to pre- and $2 \mathrm{hrs}$ postinfusion $(p=.001)$. Midazolam was also greater than ketamine at postinfusion $(p=.02)$. Within the drug $\mathrm{x}$ time $\mathrm{x}$ region $\left(\mathrm{F}[2,44]=1.72, p=.19, \eta \mathrm{p}^{2}=.07\right)$ interaction, power was reduced from pre- to postinfusion for midazolam at prefrontal $(p=.002)$, frontal $(p=.0001)$, and central $(p=.0001)$ regions, while for ketamine, power was reduced at postinfusion as compared to preinfusion for prefrontal $(p=.03)$, frontal $(p=.005)$, central $(p=.001)$, parietal $(p=.0001)$, and occipital $(p=.02)$ and $2 \mathrm{hrs}$ postinfusion for prefrontal $(p=.003)$, frontal $(p=.001)$, central $(p=.001)$, and parietal $(p=.001)$. Midazolam was also greater than ketamine at postinfusion for central $(p=.03)$ and parietal $(p=.02)$ sites.

\subsection{Theta Total}

Main effects of drug condition $\left(\mathrm{F}[1,22]=7.73, p=.01, \eta \mathrm{p}^{2}=.26\right)$ and time $(\mathrm{F}[2,44]=$ $\left.11.21, p=.0001, \eta p^{2}=.34\right)$ were found for thetaT. Power in the midazolam session was greater than with ketamine $(p=.01)$; thetaT power postinfusion was reduced as compared to pre- $(p=$ $.0001)$ and $2 \mathrm{hrs}$ postinfusion $(p=.006)$. Within the planned drug $\mathrm{x}$ time $(\mathrm{F}[2,44]=2.40, p=.10$, $\left.\eta p^{2}=.01\right)$ interaction, power was reduced from pre- to postinfusion for midazolam $(p=.04)$, while for ketamine postinfusion power was reduced as compared to pre- $(p=.003)$ and $2 \mathrm{hrs}$ postinfusion $(\mathrm{p}=.0001)$. Midazolam was also greater than ketamine at postinfusion $(p=.004)$. Average thetaT power and topographical headmaps at each time point and drug condition are displayed in Figure 2.5. 

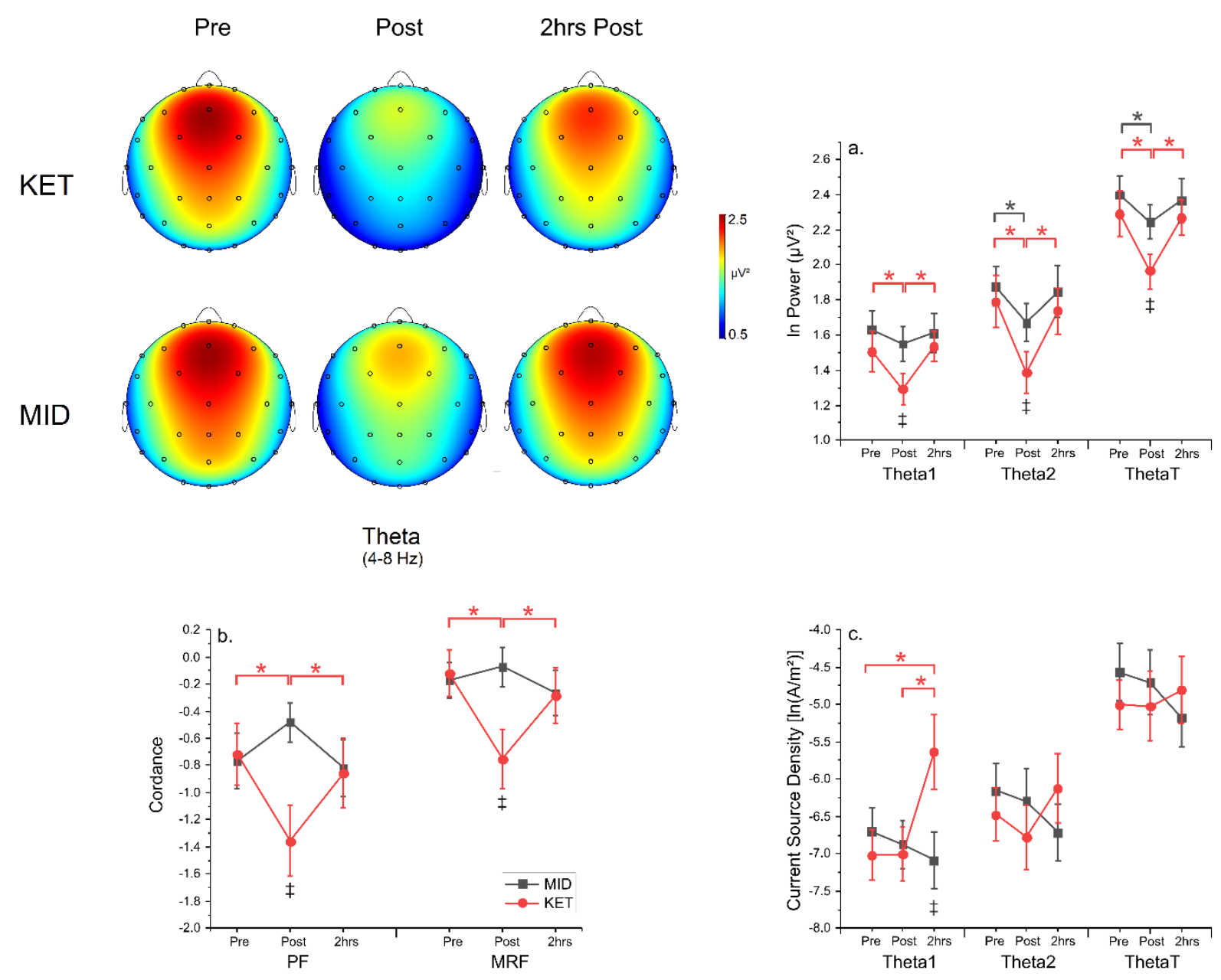

Figure 2.5. Average theta power $\left(\mu \mathrm{V}^{2}\right) \pm$ S.E. and topographical headmaps at each time point and drug condition. $*=$ time comparison, $\ddagger=$ drug condition comparison, $\mathrm{p}<.05$

\subsection{FMT theta}

Main effects of drug condition $\left(\mathrm{F}[1,22]=8.38, p=.008, \eta \mathrm{p}^{2}=.28\right)$ and time $(\mathrm{F}[2,44]=$ 7.53, $\left.p=.002, \eta p^{2}=.26\right)$ were found for FMT1 power. Power in the midazolam session was greater than with ketamine $(p=.008)$ FMT1 power postinfusion was reduced as compared to pre- $(\mathrm{p}=.006)$ and $2 \mathrm{hrs}$ postinfusion $(p=.02)$. Within the planned drug $\mathrm{x}$ time $(\mathrm{F}[2,44]=1.93, p$ $\left.=.16, \eta \mathrm{p}^{2}=.08\right)$ interaction, power was reduced from preinfusion as compared to postinfusion $(p$ 
$=.03)$ and $2 \mathrm{hrs}$ postinfusion $(p=.0001)$ for ketamine. Midazolam was also greater than ketamine at postinfusion $(p=.008)$.

Main effects of drug condition $\left(\mathrm{F}[1,22]=6.57, p=.02, \eta \mathrm{p}^{2}=.23\right)$ and time $(\mathrm{F}[2,44]=$ 22.84, $p=.0001, \eta p^{2}=.51$ ) were found for FMT2 power. Power in the midazolam session was greater than with ketamine $(p=.02)$; FMT2 power postinfusion was reduced as compared to pre$(p=.0001)$ and $2 \mathrm{hrs}$ postinfusion $(p=.0001)$. Within the planned drug $\mathrm{x}$ time $(\mathrm{F}(1.6,34.8]=$ $\left.1.76, p=.19, \eta p^{2}=.07\right)$ interaction, power was reduced from pre- to postinfusion for midazolam $(p=.001)$ and at postinfusion as compared to pre- $(p=.001)$ and $2 \mathrm{hrs}$ postinfusion $(p=.001)$ for ketamine. Midazolam was also greater than ketamine at postinfusion $(p=.02)$.

Main effects of drug condition $\left(\mathrm{F}[1,22]=9.01, p=.007, \eta \mathrm{p}^{2}=.29\right)$ and time $(\mathrm{F}[2,44]=$ $16.51, p=.0001, \eta p^{2}=.43$ ) were found for FMTT power. Power in the midazolam session was greater than with ketamine $(p=.007)$; FMTT power postinfusion was reduced as compared to pre- $(p=.0001)$ and $2 \mathrm{hrs}$ postinfusion $(p=.001)$. Within the planned drug $\mathrm{x}$ time $(\mathrm{F}[1.5,33.8]=$ $\left.2.10, p=.15, \eta p^{2}=.09\right)$ interaction, power was reduced from pre- to postinfusion for midazolam $(p=.008)$ and at postinfusion as compared to pre- $(p=.004)$ and $2 \mathrm{hrs}$ postinfusion $(p=.0001)$ for ketamine. Midazolam was also greater than ketamine at postinfusion $(p=.007)$.

\subsection{Theta Cordance (PF, MRF)}

A main interaction effect of drug $\mathrm{x}$ time $\left(\mathrm{F}[1.6,35.2]=6.25, p=.008, \eta \mathrm{p}^{2}=.22\right)$ was found for PF theta cordance. PF cordance values were reduced from pre- to postinfusion $(p=$ $.005)$ in the ketamine session only, and midazolam was greater than ketamine at postinfusion ( $p$ $=.003)$. A similar main interaction effect of drug $\mathrm{x}$ time $\left(\mathrm{F}[1.5,32.2]=6.53, p=.008, \eta \mathrm{p}^{2}=.23\right)$ was found for MRF theta cordance. MRF cordance values were reduced from pre- to postinfusion $(p=.002)$ in the ketamine session only, and midazolam was greater than ketamine 
at postinfusion $(p=.005)$. Average theta cordance values at each time point and drug condition are displayed in Figure 2.5.

\subsection{Theta ACC}

A main interaction effect of drug $\mathrm{x}$ time $\mathrm{x} \mathrm{BA}\left(\mathrm{F}[4,88]=2.62, p=.04, \eta p^{2}=.11\right)$ was found for theta 1 ACC current density. Current density values were lower $2 \mathrm{hrs}$ postinfusion as compared to pre- $(p=.02)$ and postinfusion $(p=.03)$ in the ketamine session for BA25 (sgACC) only. Midazolam was also greater than ketamine at $2 \mathrm{hrs}$ postinfusion $(p=.01)$. No main effects of drug, time, or drug $\mathrm{x}$ time interactions were found for theta 2 and thetaT current density. Average ln-transformed ACC values at each time point and drug condition are displayed in

\section{Figure 2.5.}

\subsubsection{Alpha}

\subsection{Alpha1}

A main effect of time $\left(\mathrm{F}[2,44]=19.76, p=.0001, \eta p^{2}=.47\right)$ was found for alpha1 power, with postinfusion values reduced as compared to pre- $(p=.0001)$ and $2 \mathrm{hrs}$ postinfusion $(p=$ 0.006). Within a planned drug $\mathrm{x}$ time $\left(\mathrm{F}[1.4,30.7]=19.76, p=.65, \eta \mathrm{p}^{2}=.01\right)$ interaction, alpha1 was reduced from pre- to postinfusion $(p=.008)$ in the midazolam session, while power was reduced postinfusion as compared to pre- $(p=.001)$ and $2 \mathrm{hrs}$ postinfusion $(p=.007)$.

\subsection{Alpha2}

A main effect of time $\left(\mathrm{F}[2,44]=11.60, p=.0001, \eta p^{2}=.35\right)$ was found for alpha2 power, with postinfusion values reduced as compared to pre- $(p=.0001)$ and $2 \mathrm{hrs}$ postinfusion $(p=$ 0.02). Within a planned drug $\mathrm{x}$ time $\left(\mathrm{F}[1.5,33.6]=.23, p=.74, \eta \mathrm{p}^{2}=.01\right)$ interaction, power was reduced from pre- to postinfusion $(p=.0001)$ and $2 \mathrm{hrs}$ postinfusion $(p=.04)$ for midazolam. The same differences were found in the ketamine session, with power reduced from pre- to 
postinfusion $(p=.02)$ and $2 \mathrm{hrs}$ postinfusion $(p=.04)$. Within the drug $\mathrm{x}$ time $\mathrm{x}$ region $\left(\mathrm{F}[2.9,62.8]=4.13, p=.01, \eta \mathrm{p}^{2}=.16\right)$ interaction, power was reduced from pre- to postinfusion for midazolam for all regions (prefrontal $[p=.0001]$, frontal $[p=.0001]$, central $[p=.0001]$, parietal $[p=.0001]$, occipital $[p=.001])$ and from pre- to $2 \mathrm{hrs}$ postinfusion at prefrontal $(p=$ $.02)$, frontal $(p=.04)$, and central $(p=.04)$ regions. For ketamine, power was reduced from preto postinfusion for prefrontal $(p=.006)$, frontal $(p=.003)$, central $(p=.02)$, and parietal $(p=$ $.02)$ regions, and from pre- to $2 \mathrm{hrs}$ postinfusion for prefrontal $(p=.03)$, frontal $(p=.02)$. Midazolam was also greater than ketamine at postinfusion for prefrontal $(p=.02)$ and frontal $(p$ $=.02)$ sites.

\subsection{Alpha Total}

A main effect of time $\left(\mathrm{F}[2,44]=17.48, p=.0001, \eta \mathrm{p}^{2}=.44\right)$ was found for alphaT power, with postinfusion values reduced as compared to pre- $(p=.0001)$ and $2 \mathrm{hrs}$ postinfusion $(p=0.03)$. Within a planned drug $\mathrm{x}$ time $\left(\mathrm{F}[1.5,33.4]=.30, p=.68, \eta \mathrm{p}^{2}=.01\right)$ interaction, alphaT was reduced from pre- to postinfusion $(p=.0001)$ in the midazolam session, while power was reduced postinfusion as compared to pre- $(p=.001)$ and $2 \mathrm{hrs}$ postinfusion $(p=.02)$. Average AlphaT power and topographical headmaps at each time point and drug condition are displayed in Figure 2.6.

\subsection{Alpha Asymmetry}

No main effects of drug condition or time were observed for alpha1, alpha2, or alphaT asymmetry. There were also no significant effects within a planned drug $\mathrm{x}$ time comparison. Average alpha asymmetry values at each time point and drug condition are displayed in Figure

\section{6.}



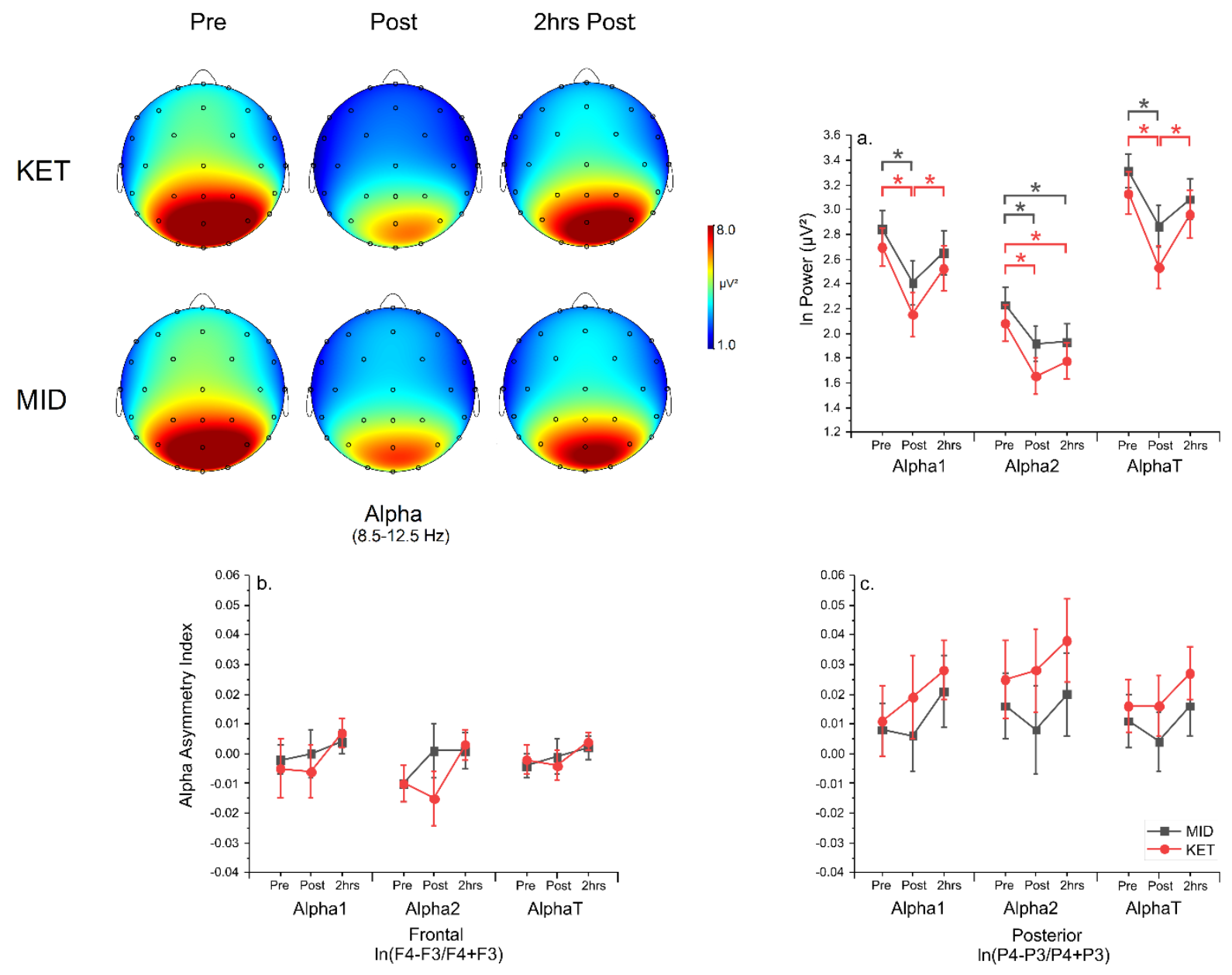

Figure 6. Average alpha power $\left(\mu \mathrm{V}^{2}\right) \pm$ S.E. and topographical headmaps at each time point and drug condition. $*=$ time comparison, $\$=$ drug condition comparison, $\mathrm{p}<.05$

\subsubsection{Beta}

A main effect of drug $\left(\mathrm{F}[1,22]=4.90, p=.04, \eta \mathrm{p}^{2}=.18\right)$ was found for betaT power, with greater power in the midazolam than in the ketamine session $(p=.04)$. Within the drug $\mathrm{x}$ time $\mathrm{x}$ region $\left(\mathrm{F}[35,76.5]=4.77, p=.003, \eta \mathrm{p}^{2}=.18\right)$ interaction, power was reduced postinfusion in two regions as compared to pre- (central $[p=.003]$, parietal $[p=.005])$ and $2 \mathrm{hrs}$ postinfusion (central $[p=.003]$, parietal $[p=.04])$. Midazolam was also greater than ketamine at 
postinfusion for central $(p=.03)$ regions. Average betaT and topographical headmaps at each time point and drug condition are displayed in Figure 2.7.

a.
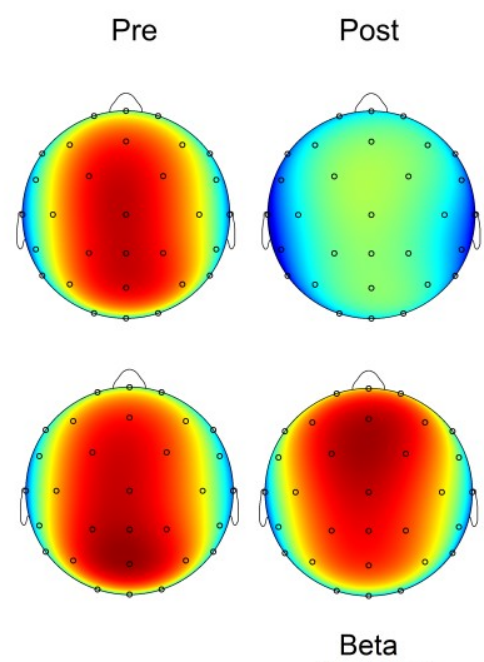
$(12.5-30 \mathrm{~Hz})$

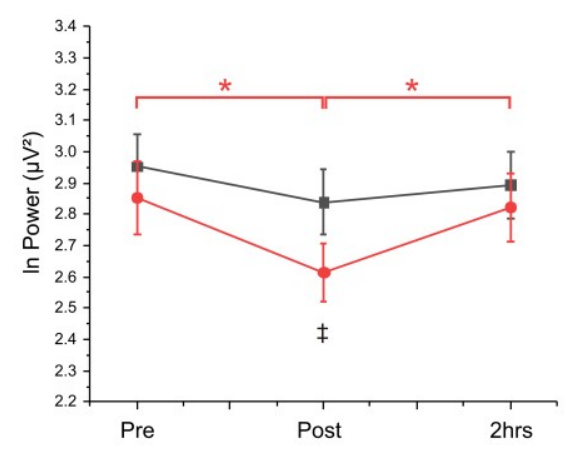

b.
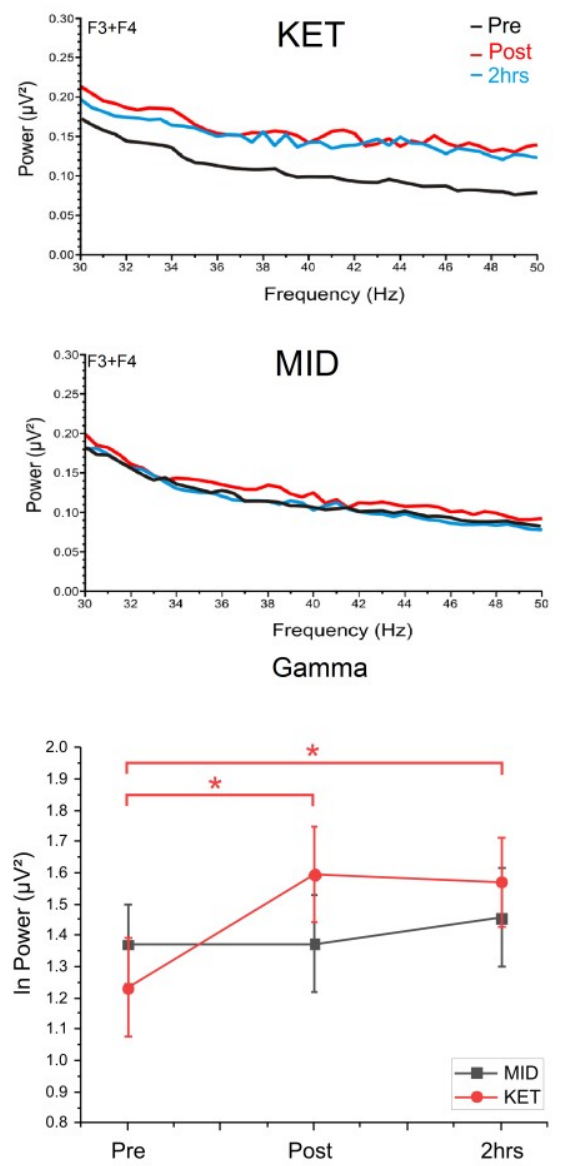

Figure 2.7. a. Average central-parietal beta power $\left(\mu \mathrm{V}^{2}\right)$ and topographical headmaps at each time point and drug condition and $\mathbf{b}$. Frontal $(\mathrm{F} 3, \mathrm{~F} 4)$ gamma spectral plots $\left(\mu \mathrm{V}^{2}\right) \pm$ S.E. $*=$ time comparison, $\$=$ drug condition comparison, $\mathrm{p}<.05$

\subsubsection{Gamma}

Main interaction effects of drug $\mathrm{x}$ time $\left(\mathrm{F}[2,44]=5.23, p=.009, \eta \mathrm{p}^{2}=.19\right)$ and drug $\mathrm{x}$ time $\mathrm{x}$ region $\left(\mathrm{F}[8,176]=2.08, p=.04, \eta p^{2}=.09\right)$ were found for gamma power. Within the drug $\mathrm{x}$ time interaction, power was increased postinfusion as compared to pre- $(p=.02)$ and $2 \mathrm{hrs}$ 
postinfusion $(p=.03)$. Ketamine was also greater than midazolam at postinfusion $(p=.01)$.

Within the drug $\mathrm{x}$ time $\mathrm{x}$ region interaction, gamma power was significantly increased postinfusion as compared to pre- $(p=.02)$ and $2 \mathrm{hrs}$ postinfusion $(p=.01)$ in the frontal region only. Average gamma and spectral plots for frontal sites at each time point and drug condition are displayed in Figure 2.7.

The fixed effects models did not result in significant interactions between baseline frontal gamma power and $\Delta 2 \mathrm{hrs}$ or $\Delta 1 \mathrm{~d}$ postinfusion.

\subsubsection{Relationship between Baseline and Ketamine-induced Changes in EEG Measures} with Acute and Sustained Change in MADRS/SI

\subsubsection{Baseline EEG and Changes in MADRS/SI}

Early (2hrs, 1 d post). Correlations indicated a negative relationship between baseline rACC theta1 $(r=-.43, p=.04, \mathrm{~N}=23), \operatorname{sgACC}$ theta2 $(r=-.50, p=.02, \mathrm{~N}=23)$, rACC theta2 $(r$ $=-.55, p=.007, \mathrm{~N}=23)$, and $\operatorname{sgACC}$ thetaT $(r=-.43, p=.04, \mathrm{~N}=23)$ with $\Delta 1 \mathrm{~d}$ postinfusion MADRS total score. A negative relationship between baseline $\operatorname{sgACC}$ theta2 $(r=-.47, p=.02, \mathrm{~N}$ $=23)$ and $\mathrm{rACC}$ theta2 $(r=-.51, p=.01, \mathrm{~N}=23)$ with $\Delta 1 \mathrm{~d}$ postinfusion MADRS SI was also observed (Figure 2.8).

Sustained (phases 2,3). Negative relationships were observed between baseline rACC theta1 and $\Delta \mathrm{Ph} 2$ MADRS $(r=-.48, p=.03, \mathrm{~N}=22)$, between alphaT frontal asymmetry Band $\Delta \mathrm{Ph} 3 \mathrm{MADRS}(r=-.58, p=.04, \mathrm{~N}=13)$, and between alphaT parieto-occipital and $\Delta \mathrm{Ph} 3$ MADRS SI $(r=-.58, p=.04, \mathrm{~N}=13)$ (Figure 2.8). 


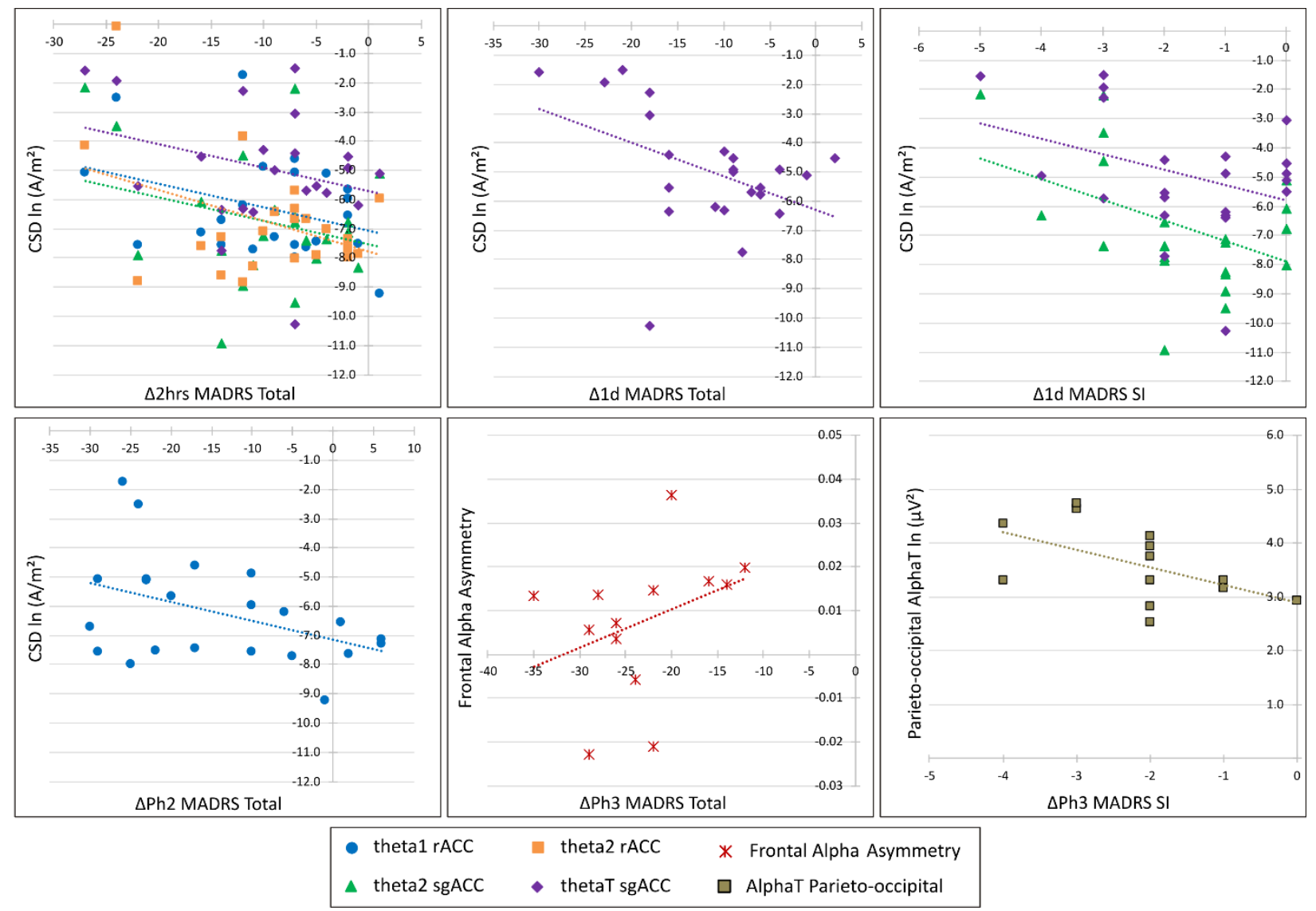

Figure 2.8. Scatterplots of significant correlations between baseline current source density (CSD) anterior cingulate cortex (ACC)-localized activity, frontal alpha asymmetry, and alpha total (AlphaT) parieto-occipital power $\left(\mu \mathrm{V}^{2}\right)$ with change in Montgomery-Åsberg Depression Rating Scale (MADRS) and MADRS item 10 (suicidal ideation, SI) at 2 hours $(\Delta 2 \mathrm{hrs}$ ) and one day $(\Delta 1 \mathrm{~d})$ postinfusion, and change from baseline to end of phase $2(\Delta \mathrm{Ph} 2)$, and end of phase 3 $(\Delta \mathrm{Ph} 3)$.

\subsubsection{Ketamine-induced Changes in EEG and in MADRS/SI}

Early (2hrs, 1 d post). Correlations indicated a positive relationship between $\Delta$ postinfusion FMT1 $(r=.42, p=.04, \mathrm{~N}=23)$ and PF theta cordance $(r=.53, p=.01, \mathrm{~N}=23)$ with $\Delta 2 \mathrm{hrs}$ postinfusion MADRS, while a negative relationship was observed between $\Delta$ postinfusion $(r=-.44, p=.03, \mathrm{~N}=23)$ and $\Delta 2 \mathrm{hrs}$ postinfusion $(r=-.46, p=.03, \mathrm{~N}=23)$ frontal gamma with $\Delta 2 \mathrm{hrs}$ postinfusion MADRS. A positive relationship between $\Delta$ postinfusion FMT1 $(r=.48, p=.02, \mathrm{~N}=23)$ was also found with $\Delta 1 \mathrm{~d}$ postinfusion MADRS (Figure 9). 

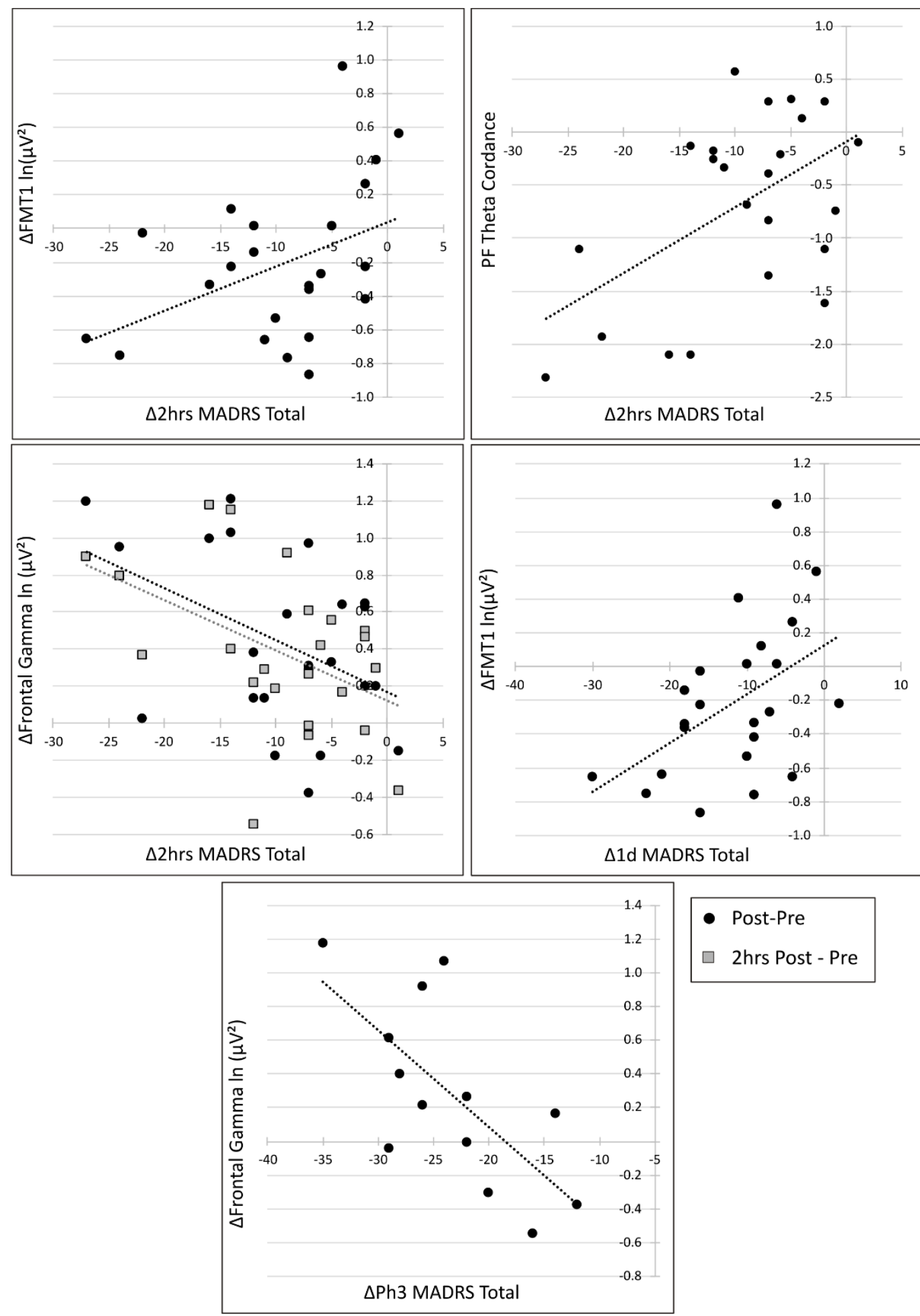

- Post-Pre

$\square \quad$ 2hrs Post - Pre

Figure 2.9. Scatterplots of significant correlations between change in prefrontal (PF) theta cordance, frontal midline theta1 (FMT1) power $\left(\mu \mathrm{V}^{2}\right)$, and frontal gamma power with change in Montgomery-Åsberg Depression Rating Scale (MADRS) and MADRS item 10 (suicidal ideation, SI) at 2 hours ( $\Delta 2 \mathrm{hrs})$ and one day $(\Delta 1 \mathrm{~d})$ postinfusion, and change from baseline to end of phase $3(\Delta \mathrm{Ph} 3)$. 
Negative relationships between $\Delta$ postinfusion alphal $(r=-.42, p=.04, \mathrm{~N}=23)$ and $\Delta$ postinfusion alphaT power $(r=-.44, p=.03, \mathrm{~N}=23)$ with $\Delta 2 \mathrm{hrs}$ postinfusion MADRS SI, as well as between $\Delta$ postinfusion alpha1 $(r=-.49, p=.01, \mathrm{~N}=23)$ and $\Delta$ postinfusion alphaT $(r=$ $-.48, p=.02, \mathrm{~N}=23$ ) with $\Delta 1 \mathrm{~d}$ MADRS SI were observed (Figure 2.10).

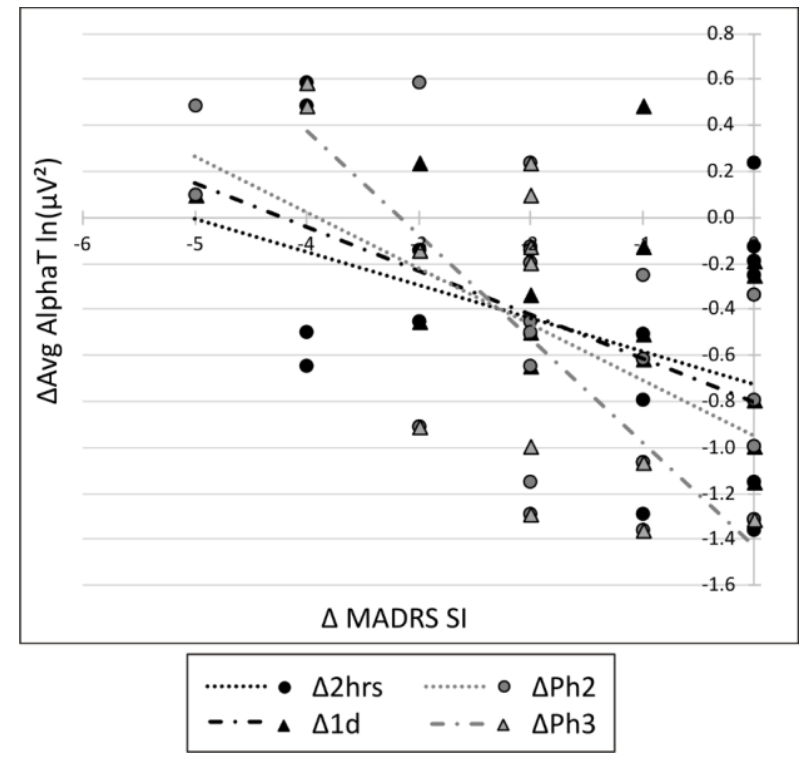

Figure 2.10. Scatterplots of significant correlations between change in alpha total (AlphaT) power $\left(\mu \mathrm{V}^{2}\right)$ with change in Montgomery-Åsberg Depression Rating Scale (MADRS) item 10 (suicidal ideation, SI) at 2 hours $(\Delta 2 \mathrm{hrs})$ and one day $(\Delta 1 \mathrm{~d})$ postinfusion, and change from baseline to end of phase $2(\Delta \mathrm{Ph} 2)$, and end of phase $3(\Delta \mathrm{Ph} 3)$. 
Sustained (phase 2, phase 3). Negative relationships between $\Delta$ postinfusion alpha1 ( $r=$ $-.57, p=.006, \mathrm{~N}=23)$ and $\Delta$ postinfusion alphaT power $(r=-.58, p=.004, \mathrm{~N}=23)$ with $\Delta \mathrm{Ph} 2$ MADRS SI, and between alpha1 $(r=-.72, p=.006, \mathrm{~N}=13)$ and $\Delta$ postinfusion alphaT power $(r$ $=-.72, p=.006, \mathrm{~N}=13$ ) with $\triangle \mathrm{Ph} 3$ MADRS SI, were observed (Figure 2.10). A negative relationship between $\Delta 2 \mathrm{hrs}$ postinfusion frontal gamma change $(r=-.69, p=.01, \mathrm{~N}=13)$ with $\triangle \mathrm{Ph} 3 \mathrm{MADRS}$ was also found (Figure 2.9).

\subsubsection{Ketamine-induced Changes in EEG and in BPRS-P}

A negative correlation was found between $\Delta$ postinfusion parietal alphaT and $\triangle \mathrm{BPRS}-\mathrm{P}$ symptoms $(r=-.43, p=.04, \mathrm{~N}=22)$.

\subsubsection{Prediction of Acute and Sustained Response to Ketamine}

Models containing only EEG measures as well as models containing EEG and MADRS predictors were examined. The model parameters (unstandardized Beta coefficient $[B]$, standard error $[$ S.E.)], t-statistic $[t]$ and significance $[p])$ for all regression analyses are reported in Table

\section{3.}

MADRS Total. Significant stepwise regression models containing only EEG measures were found for the prediction of $\Delta 2 \mathrm{hrs}, \Delta 1 \mathrm{~d}, \Delta \mathrm{Ph} 2$, and $\Delta \mathrm{Ph} 3$. Regarding early response, a prediction of $\Delta 2 \mathrm{hrs}$ MADRS from $\Delta$ postinfusion FMT1, $\Delta$ postinfusion PF theta cordance, $\Delta$ and $\Delta 2 \mathrm{hrs}$ postinfusion frontal gamma resulted in one model $\left(\mathrm{F}[1,22]=8.03, p=.01, \mathrm{R}^{2}=.28\right)$ containing $\Delta$ postinfusion PF theta cordance. A prediction of $\triangle 1 \mathrm{~d}$ MADRS from baseline rACC theta1, sgACC theta2, rACC theta2, sgACC thetaT, and $\triangle$ postinfusion FMT1 also resulted in one model $\left(\mathrm{F}[1,22]=9.03, p=.007, \mathrm{R}^{2}=.30\right)$ containing baseline sgACC theta2. Regarding sustained response, a prediction of $\triangle \mathrm{Ph} 2 \mathrm{MADRS}$ from baseline rACC theta1, $\Delta 2 \mathrm{hrs}$ postinfusion FMT1, $\Delta 2 \mathrm{hrs} \mathrm{MADRS,} \mathrm{and} \Delta 1 \mathrm{~d}$ MADRS resulted in one model $(\mathrm{F}[1,21]=5.83, p$ 
$=.03, \mathrm{R}^{2}=.23$ ) containing rACC theta1, while for a prediction of $\triangle \mathrm{Ph} 3$ MADRS from baseline alphaT frontal asymmetry, $\Delta 2 \mathrm{hrs}$ postinfusion frontal gamma, $\Delta 2 \mathrm{hrs}$ MADRS, and $\Delta 1 \mathrm{~d}$ MADRS resulted in one model $\left(\mathrm{F}[1,12]=6.22, p=.03, \mathrm{R}^{2}=.36\right)$ containing $\Delta 2 \mathrm{hrs}$ frontal gamma power. With the addition of baseline total MADRS score (to all) and $\Delta 2 \mathrm{hrs}$ (to $\Delta 1 \mathrm{~d}, \Delta \mathrm{Ph} 2, \Delta \mathrm{Ph} 3$ ) and $\Delta 1 \mathrm{~d}$ MADRS (to $\Delta \mathrm{Ph} 2, \Delta \mathrm{Ph} 3$ ), the model predicting $\Delta 1 \mathrm{~d}$ was altered: the analysis produced two models, containing 1) $\Delta 2 \mathrm{hrs}$ MADRS $\left(\mathrm{F}[1,22]=17.03, p=.0001, \mathrm{R}^{2}=.45\right)$, and 2) $\Delta 2 \mathrm{hrs}$ MADRS $+\operatorname{sgACC}$ theta2 $\left(\mathrm{F}[2,22]=12.41, p=.0001, \mathrm{R}^{2}=.55\right)$. The other models remained unchanged.

MADRS SI. Significant stepwise regression models containing only EEG measures were found for the prediction of $\Delta 2 \mathrm{hrs}, \Delta 1 \mathrm{~d}$, and $\Delta \mathrm{Ph} 3$. Regarding early response, a prediction of $\Delta 2 \mathrm{hrs}$ MADRS SI from $\Delta$ postinfusion Alpha1 power and $\Delta$ postinfusion average alphaT power resulted in one model $\left(\mathrm{F}[1,22]=5.13, p=.03, \mathrm{R}^{2}=.20\right)$ containing $\Delta$ postinfusion average alphaT power. The prediction of $\triangle 1 \mathrm{~d}$ MADRS SI from baseline $\mathrm{rACC}$ and $\mathrm{sgACC}$ theta2, $\Delta$ postinfusion average Alpha1, and average alphaT resulted in two models: $(\mathrm{F}[1,22]=7.46, p=$ $\left..007, \mathrm{R}^{2}=.26\right)$ with $\mathrm{rACC}$ theta2, and $\left(\mathrm{F}[1,22]=9.46, p=.001, \mathrm{R}^{2}=.49\right)$ with $\Delta$ postinfusion average alphaT and rACC theta2. Regarding sustained response, a prediction of $\triangle \mathrm{Ph} 3 \mathrm{MADRS}$ SI from baseline alphaT parieto-occipital, $\Delta$ postinfusion alpha1, $\Delta$ postinfusion alphaT power resulted in one model $\left(\mathrm{F}[1,21]=10.36, p=.004, \mathrm{R}^{2}=.34\right)$ containing $\Delta$ postinfusion alphaT power. The prediction of $\Delta \mathrm{Ph} 2 \mathrm{MADRS}$ SI from $\Delta$ postinfusion alpha1, $\Delta$ postinfusion alphaT did not yield a significant model.

With the addition of baseline MADRS SI (to all) and $\Delta 2 \mathrm{hrs}$ (to $\Delta 1, \Delta \mathrm{Ph} 2, \Delta \mathrm{Ph} 3$ ) and $\Delta 1 \mathrm{~d}$ MADRS SI (to $\Delta \mathrm{Ph} 2, \Delta \mathrm{Ph} 3)$, all models were altered. The models predicting $\Delta 2 \mathrm{hrs}(\mathrm{F}[1,22]=$ $\left.17.03, p=.0001, \mathrm{R}^{2}=.45\right)$ and $\Delta \mathrm{Ph} 3\left(\mathrm{~F}[1,12]=20.57, p=.001, \mathrm{R}^{2}=.65\right)$ had baseline MADRS 
SI entered as their only predictor. The model predicting $\Delta \mathrm{Ph} 2\left(\mathrm{~F}[1,21]=24.17, p=.0001, \mathrm{R}^{2}=\right.$ .55 ), was significant with $\Delta 2 \mathrm{hrs}$ MADRS SI as a predictor. The model predicting $\Delta 1 \mathrm{~d}$ was altered: the analysis produced two models, containing 1) $\Delta 2 \mathrm{hrs}$ MADRS SI $(\mathrm{F}[1,22]=20.45, p=$ $\left..0001, \mathrm{R}^{2}=.49\right)$, and 2) $\Delta 2 \mathrm{hrs}$ MADRS $+\mathrm{rACC}$ theta2 $\left(\mathrm{F}[2,22]=15.99, p=.0001, \mathrm{R}^{2}=.62\right)$.

Table 2.3. Model parameters for regression analyses

\begin{tabular}{|c|c|c|c|c|c|c|}
\hline \multicolumn{7}{|c|}{ MADRS Total } \\
\hline Model & & Independent variables & $\boldsymbol{B}$ & S.E. & $\mathbf{t}$ & $p$ \\
\hline \multirow{2}{*}{$\begin{array}{l}\Delta 2 \mathrm{hrs} \\
\text { MADRS }\end{array}$} & EEG & $\Delta$ post PF theta cordance & 4.49 & 1.58 & 2.83 & .010 \\
\hline & EEG + MADRS & $\Delta$ post PF theta cordance & 4.49 & 1.58 & 2.83 & .010 \\
\hline \multirow{2}{*}{$\begin{array}{l}\Delta 1 \mathrm{~d} \\
\text { MADRS }\end{array}$} & EEG & Baseline sgACC theta2 & -2.13 & .71 & -3.01 & .007 \\
\hline & EEG + MADRS & $\begin{array}{l}\triangle 2 \mathrm{hrs} \text { MADRS }+ \\
\text { Baseline sgACC theta2 }\end{array}$ & $\begin{array}{c}.59 \\
-1.21\end{array}$ & $\begin{array}{l}.16 \\
.55\end{array}$ & $\begin{array}{r}3.69 \\
-2.18\end{array}$ & $\begin{array}{l}.001 \\
.04\end{array}$ \\
\hline \multirow{2}{*}{$\begin{array}{l}\Delta P 2 \\
\text { MADRS }\end{array}$} & EEG & Baseline rACC theta1 & -3.08 & 1.27 & -2.42 & .03 \\
\hline & $\mathrm{EEG}+\mathrm{MADRS}$ & Baseline rACC theta1 & -3.08 & 1.27 & -2.42 & .03 \\
\hline \multirow{2}{*}{$\begin{array}{l}\Delta P h 3 \\
\text { MADRS }\end{array}$} & EEG & $\Delta 2 \mathrm{hrs}$ frontal gamma & -6.29 & 2.52 & -2.49 & .03 \\
\hline & EEG + MADRS & $\Delta 2 \mathrm{hrs}$ frontal gamma & -6.29 & 2.52 & -2.49 & .03 \\
\hline \multicolumn{7}{|c|}{ MADRS SI } \\
\hline Model & & Independent variables & $\boldsymbol{B}$ & SE & $\mathbf{t}$ & $p$ \\
\hline \multirow{2}{*}{$\begin{array}{l}\Delta 2 \mathrm{hrs} \\
\text { MADRS SI }\end{array}$} & EEG & $\Delta$ post avg AlphaT & -1.36 & .60 & -2.27 & .03 \\
\hline & $\mathrm{EEG}+\mathrm{MADRS}$ & Baseline MADRS SI & -.76 & .22 & -3.42 & .003 \\
\hline \multirow[t]{2}{*}{$\begin{array}{l}\Delta 1 \mathrm{~d} \\
\text { MADRS SI }\end{array}$} & EEG & $\begin{array}{l}\Delta \text { post avg AlphaT }+ \\
\text { Baseline rACC theta2 }\end{array}$ & $\begin{array}{l}-1.19 \\
-.36\end{array}$ & $\begin{array}{l}.40 \\
.12\end{array}$ & $\begin{array}{l}-2.95 \\
-3.16\end{array}$ & $\begin{array}{l}.008 \\
.005\end{array}$ \\
\hline & EEG + MADRS & $\begin{array}{l}\triangle 2 \mathrm{hrs} \text { MADRS SI }+ \\
\text { rACC theta } 2 \text { baseline }\end{array}$ & $\begin{array}{l}.50 \\
-.26\end{array}$ & $\begin{array}{l}.12 \\
.10\end{array}$ & $\begin{array}{r}4.29 \\
-2.52\end{array}$ & $\begin{array}{c}.0001 \\
.02\end{array}$ \\
\hline \multirow{2}{*}{$\begin{array}{l}\Delta \text { Ph2 } \\
\text { MADRS SI }\end{array}$} & EEG & - & - & - & - & - \\
\hline & EEG + MADRS & $\Delta 2 \mathrm{hrs}$ MADRS SI & .59 & .12 & 4.92 & .0001 \\
\hline \multirow[t]{2}{*}{$\begin{array}{l}\Delta \text { Ph3 } \\
\text { MADRS SI }\end{array}$} & EEG & $\Delta$ post avg AlphaT & $\begin{array}{l}-1.03 \\
-.67\end{array}$ & $\begin{array}{l}.29 \\
.29\end{array}$ & $\begin{array}{l}-3.57 \\
-2.28\end{array}$ & $\begin{array}{l}.005 \\
.04\end{array}$ \\
\hline & EEG + MADRS & Baseline MADRS SI & -.78 & .17 & -4.54 & .001 \\
\hline
\end{tabular}

B, Beta coefficient; S.E., standard error 


\subsubsection{Baseline EEG Differences and Ketamine-Induced Changes between Early (1d) and}

\section{Sustained (end of Ph2) Treatment Responders}

Despite a small sample size, as most EEG prediction studies report on responders vs. non-responders, we aimed to examine baseline and ketamine-induced change variables in these groups at an early (1d) and sustained $(\mathrm{Ph} 2)$ time points, in order to compare our findings to previous work. Early responders had significantly larger theta activity at both the scalp and source level, as well as a greater ketamine-induced decrease. Sustained responders had significantly larger theta source activity and a greater decrease with ketamine (Table 2.4).

Table 2.4. Means ( \pm S.E.) for baseline variables and Mann-Whitney U-test and exact $p$ values for responders and non-responders at One Day and Phase 2.

\begin{tabular}{|l|c|c|c|c|}
\hline \multicolumn{5}{|c|}{ One Day } \\
\hline Baseline EEG Feature & Responders & Non-Responders & M-W (U) & p (exact) \\
\hline Theta2 sgACC & $-4.83(1.02)$ & $-7.44(.38)$ & $20(-2.17)$ & .03 \\
\hline Theta2 rACC & $-5.02(1.08)$ & $-7.40(.29)$ & $13(-2.66)$ & .006 \\
\hline ThetaT sgACC & $-3.14(.78)$ & $-5.59(.41)$ & $17(-2.38)$ & .02 \\
\hline ThetaT rACC & $-3.07(.86)$ & $-5.08(.38)$ & $15(-2.52)$ & .01 \\
\hline FMT2 & $2.89(.30)$ & $2.20(.13)$ & $19(-2.25)$ & .02 \\
\hline FMTT & $3.37(.25)$ & $2.69(.13)$ & $19(-2.24)$ & .02 \\
\hline \multicolumn{5}{|l|}{ One Day } \\
\hline EEG Feature & Responders & Non-Responders & M-W (U) & p (exact) \\
\hline Theta1 rACC & $-5.49(.50)$ & $-7.35(.38)$ & $21(-2.50)$ & .01 \\
\hline
\end{tabular}

ACC, anterior cingulate cortex; FMT, Frontal midline theta

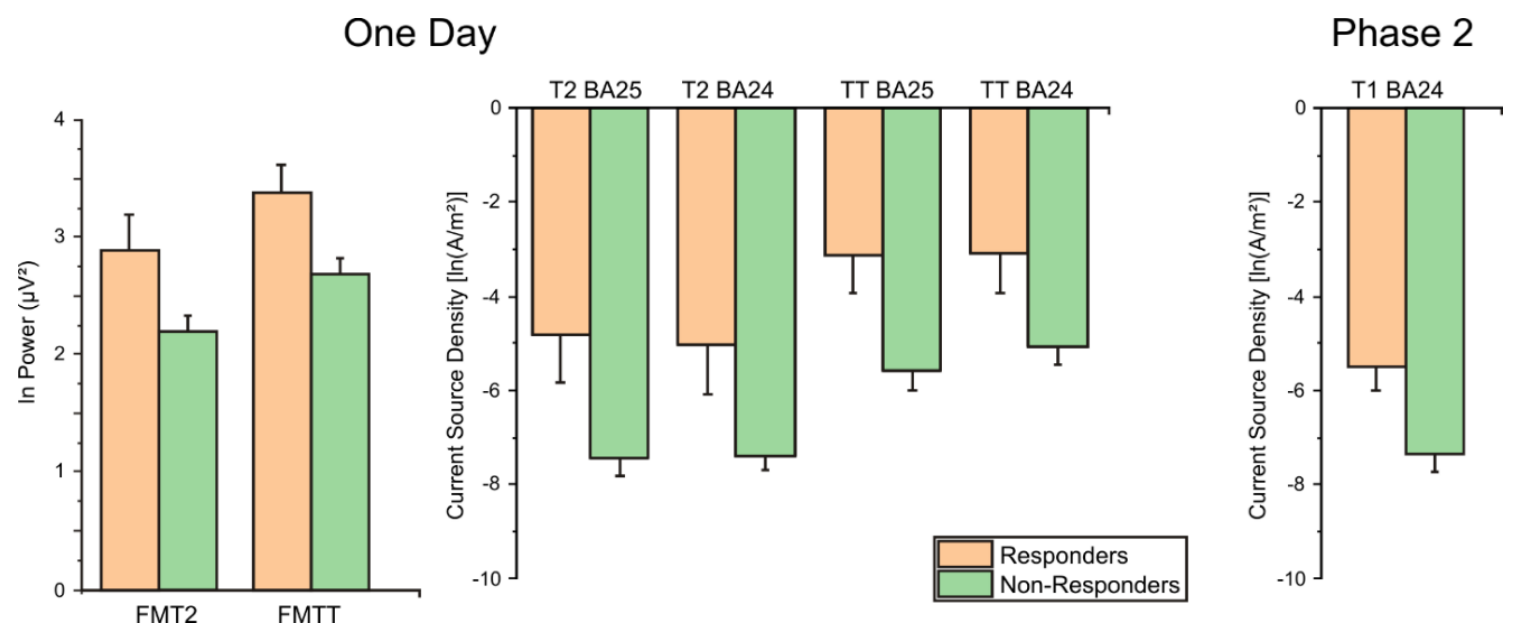

Figure 2.11. Mean ( \pm S.E.) baseline EEG features that differed in responders and non-responders at one day postinfusion and at the end of phase 2 . 


\subsection{Discussion}

This electrophysiological study aimed to examine the acute effects of sub-anesthetic ketamine (as compared to the active placebo midazolam) within the randomized, double-blind phase of a multi-phase trial examining single and repeated ketamine infusions in MDD patients with TRD. It also aimed to examine the relationship between baseline and acute ketamineinduced changes in electrophysiological measures with acute and sustained change in depressive and SI symptoms. Additionally, differences in baseline were compared within responders and non-responders at one day postinfusion (early response) and at the end of Phase 2 (sustained response).

Regional- and frequency-specific ketamine-induced changes in electrophysiological measures were observed. In a recent healthy control study examining sub-anesthetic ketamine $(0.25 \mathrm{mg} / \mathrm{kg}$ bolus dose, $0.25 \mathrm{mg} / \mathrm{kg} / \mathrm{h}$ infusion $)$, midazolam $(0.03 \mathrm{mg} / \mathrm{kg}$ bolus dose, followed by a $0.03 \mathrm{mg} / \mathrm{kg} / \mathrm{h}$ infusion), and placebo (saline), less complex changes occurred with midazolam, only finding increases in frontal delta, low beta, and fronto-central high beta activity, as well as decreases in occipital alpha and fronto-central theta power. This same study found decreases in occipital alpha, delta, and low beta and increases in frontal theta, high beta, low gamma, and whole-brain high gamma (Forsyth et al., 2018). While few studies have examined these electrophysiological changes in MDD, this same research group found ketamine-induced increases in theta, high beta, low and high gamma power, with beta and gamma power remaining elevated throughout the infusion, and theta power returning to baseline four minutes postinfusion initiation. Decreases in delta, alpha, and low beta power were observed, and remained low throughout the infusion (McMillan et al., 2020). 
Within our current study, delta band increases were observed from pre to postinfusion in the midazolam condition. This change is in line with previous work demonstrating increases in delta power during sedation (Hering et al., 1994; Numan et al., 2019) and with lower subanesthetic doses (Forsyth et al., 2018). Originating from medial cortical regions, delta power is often assessed during slow-wave sleep states, however, its activity during wake (at rest) has been suggested to function as a synchronization of cerebral activity with autonomic and homeostatic processes. This function is activated during sleep but remains suppressed when more advanced and operationally flexible processes related to higher frequencies dominate during the waking state (Knyazev, 2012). When these oscillations are disrupted (for example, with the administration of a benzodiazepine), delta power increases. Though previous studies have found decreases in delta power with sub-anesthetic doses of ketamine (de la Salle et al., 2016 [Appendix I]; Forsyth et al., 2018; Knott et al., 2006; McMillan et al., 2019; Shaw et al., 2015; Vlisides et al., 2017, 2018), none were observed in this study sample.

The measures of theta band activity (power, cordance, source-localized ACC activity) showed different patterns of changes with both ketamine and midazolam. While functional differences between resting theta 1 and theta 2 activity have not been elaborated, studies have shown sub-band specific alterations in MDD (Jaworska et al., 2012; Narushima et al., 2010; Pizzagalli et al., 2001). In the current study, midazolam did not alter theta1, but decreased theta2 (prefrontal, frontal, central) and thetaT postinfusion, while ketamine decreased theta1, theta2 (all regions), and thetaT postinfusion. At the postinfusion time point, ketamine had also decreased power to a greater degree than midazolam (all sites for theta1, thetaT, central and parietal for theta2). While there are no comparable studies of changes in theta power with low-dose midazolam administration in MDD, we can hypothesize that theta power would be decreased, 
similar to the effect of other low doses of benzodiazepines (Sampaio et al., 2008). As this study did not employ a bolus dose, our finding of decreased theta activity with ketamine is in line with previous work (de la Salle et al., 2016 [Appendix I]; Knott et al., 2006; Vlisides et al., 2017, 2018). Similarly, acute decreases in prefrontal theta cordance were observed with ketamine only; this was also observed in healthy controls receiving a sub-anesthetic infusion without a bolus (Horacek et al., 2010; Sanacora et al., 2014). A similar ketamine-induced decrease was observed in the MRF region. No changes with midazolam were found. This suggests a unique effect of ketamine in MDD that is independent of the general decrease in theta cordance observed with power values. Finally, sgACC theta1 source-localized CSD activity was found to increase with ketamine $2 \mathrm{hrs}$ postinfusion, with no significant changes observed with midazolam. Previous studies have found decreases in the $\operatorname{sgACC}$ with acute ketamine administration in healthy controls (Deakin et al., 2008; Stone et al., 2015; Wong et al., 2016). However, an increase with ketamine was observed with BOLD signal in the sgACC during an hour-long scanning session, with the activation predicting depression improvements at 24 hours and 1 week post-ketamine (though the study did not observe changes in depression symptoms immediately postinfusion, Downey et al., 2016). The authors suggest that the previously reported deactivation of sgACC after ketamine probably reflects the rapid and pronounced subjective effects evoked by the bolus-infusion method used in the previous study. Additionally, activity within the sgACC activity increased over the whole recording, so it is possible that the decrease in other studies is related to time of measurement.

Alpha power was reduced with both ketamine and midazolam, reflecting an overall decrease in arousal in both drug conditions. This is consistent with previous healthy control studies with midazolam (Berchou et al., 1986; Hotz et al., 2000; Kuizenga et al., 2001; Veselis et 
al., 1991) and sub-anesthetic ketamine (de la Salle et al., 2016 [Appendix I]; Forsyth et al., 2018; Kochs et al., 1996; McMillan et al., 2019; Muthukumaraswamy et al., 2015; Vlisides et al., 2017, 2018). Alpha power during eyes-closed resting conditions is thought to reflect a state of relaxed alertness (Zoon et al., 2013), though it has also been implicated in various cognitive processes, including attention, perception, and working memory, and may prioritize relevant sensory input (Klimesch, 1999, 2012; Van Diepen et al., 2019). In MDD, alpha power is typically increased (Bruder et al., 2008; Grin-Yatsenko et al., 2009; Jaworska et al., 2012), suggesting an overall pattern of cortical hypoactivity. We observed decreases in parietal alpha power which were correlated with greater increases in BPRS-P symptoms, suggesting that the specific positive symptoms induced by ketamine are disrupting alpha oscillations, perhaps representing a decrease in sensory input filtering. Alpha asymmetry was not significantly altered within either drug condition. This is in line with the results of the international Study to Predict Optimized Treatment in Depression (iSPOT-D), which found that frontal alpha asymmetry is a stable trait which remains unaffected following treatment with SSRI (van der Vinne et al., 2019).

Beta oscillations have traditionally been associated with the sensorimotor cortex (Kilavik et al., 2013), though recent work has also suggested a role in large-scale neural integration (Donner \& Siegel, 2011) and temporal perception (Arnal, 2012). Central-parietal beta power was reduced following acute ketamine infusion, which is consistent with previous human trials (Forsyth et al., 2018; Knott et al., 2006; McMillan et al., 2019; Muthukumaraswamy et al., 2015; Rivolta et al., 2015; Vlisides et al., 2018). While increases in beta power are typically found with midazolam administration, none were found in the current study. These increases are dosedependent, therefore it is possible that the dose employed in the current study was too low to observe any acute changes (Forsyth et al., 2018). Additionally, benzodiazepines typically alter 
lower beta range frequencies $(\sim 13.5-15.5 \mathrm{~Hz})$, while in the current study total beta power (12.5$30 \mathrm{~Hz}$ ) was examined (Breimer et al., 1990; Hotz et al., 2000).

Gamma oscillations resulting from emotional stimuli have been found to be altered in MDD (Bi et al., 2018; Lee et al., 2010; Liu et al., 2012, 2014), possibly reflecting disruptions within GABAergic inhibition (Fee et al., 2017). Ketamine-induced increases in gamma power are thought to occur through pyramidal cell disinhibition downstream of NMDA receptor antagonism, and that this increase in gamma may be involved in the reduction of depression symptoms via cortical excitation increase (Gilbert \& Zarate, 2020). We observed increases in frontal gamma power both immediately and 2 hours postinfusion. Increased resting gamma power is a consistent finding with acute sub-anesthetic ketamine administration in healthy controls (de la Salle et al., 2016 [Appendix I]; Forsyth et al., 2018; McMillan et al., 2019; Muthukumaraswamy et al., 2015; Rivolta et al., 2015; Sanacora et al., 2014; Vlisides et al., 2017, 2018) and has recently been found in MDD patients as a delayed effect with increases 6-9hrs postinfusion (Nugent et al., 2019a). Our findings of acute increases in gamma power both immediately and 2 hours postinfusion are the first to demonstrate this increase in TRD MDD patients, and complement findings of sustained gamma increases beyond ketamine's acute physiological and dissociative effects.

Certain ketamine-induced changes in EEG measures were found to be related to, and in certain cases predictive of, decreases in depressive symptoms and SI. Notably, theta, alpha, and gamma activity demonstrated the most relation to decreases in depressive symptoms, though at differing points of symptom assessments. Early decreases in depressive symptoms $2 \mathrm{hrs}$ postinfusion were associated with acute change in FMT1, PF cordance, and frontal gamma power, while at one day postinfusion, the strongest relationships were with baseline source- 
localized theta ACC activity (theta1/2 rACC and theta2/T sgACC) and decrease in FMT1 activity. At the end of phase 2, baseline theta1 (rACC) and frontal alpha asymmetry were associated with decrease in symptoms, while increase in frontal gamma was related to decrease in symptoms at the end of Phase 3. Decreases in SI symptoms were most associated with measures of alpha1 and alphaT power.

Findings with baseline and change in theta oscillations have been observed with traditional antidepressants, though conflicting results have been found (Baskaran et al., 2018; Iosifescu et al., 2009; Knott et al., 2000, 1996; Spronk et al., 2011; Tenke et al., 2011). With ketamine, the findings of McMillan et al. (2020) were an increase in frontal theta power, with an observation of a trend towards increased frontal theta power relating to a larger decrease in MADRS score at one-day postinfusion. In the current study, we found decreases in measures of theta oscillations, as well as a relationship between decreases in theta and decreases in depressive symptoms. Cao et al. (2019) found that treatment responders exhibited decreases in prefrontal theta cordance 240 minutes ketamine postinfusion. Within our study, we observed an immediate decrease in theta cordance which was related to decreases in depressive symptoms at $2 \mathrm{hrs,}$ though no differences in cordance values at $2 \mathrm{hrs}$. Given the differing recording time points, it is difficult to parse apart the time course of change in theta cordance and more work is required.

Correlations were observed with source-localized baseline ACC activity at both early (one day postinfusion) and sustained response time points (end of Phase 2). As well, early and sustained responders had higher levels of theta as compared to non-responders. Increased resting activity in the rACC has been consistently shown to be related to treatment response to many antidepressant treatments (pharmacotherapy, transcranial magnetic stimulation, sleep deprivation, (Fu et al., 2013; Pizzagalli, 2011), and that rACC theta activity is related to 
metabolism (Pizzagalli et al., 2003). Changes within the sgACC have been associated with treatment response to CBT and pharmacotherapy (Kennedy et al., 2007). In the current study, increases in sgACC thetal activity were found $2 \mathrm{hrs}$ postinfusion; however, there was no relationship to decrease in depressive symptoms. It is possible that further increases may have been observed with a later recording (one day postinfusion, end of phase 2) which may then have elucidated a relationship with decrease in symptoms.

Regarding gamma power changes, Nugent et al. (2019a) observed that baseline gamma levels within widespread regions, which included the central executive (CEN), salience (SN), and default mode (DMN) networks, were found to moderate the relationship between change in gamma power post-ketamine ( $\sim-9 \mathrm{hrs})$ and antidepressant response, with patients who had lower baseline levels exhibiting greater increases in gamma and greater decreases in depressive symptoms (from -60 to 40 mins postinfusion). Patients with higher baseline levels and large increases in gamma power exhibited smaller decreases in depressive symptoms (Nugent et al., 2019a). We did not observe baseline moderation effects in our analysis - this may be related to the difference in time of recording (immediately and $2 \mathrm{hrs}$ postinfusion vs. $6-9 \mathrm{hrs}$ postinfusion) as well as the time of recording for baseline values (first recording, same day as infusion vs. 1-2 days preinfusion). One possible interpretation is that baseline influences the sustained vs. immediate increase in gamma power. Additionally, we were looking at one scalp-level region (frontal) vs. activity within larger-scale networks. In the current study, patients with greater acute change in gamma power exhibited greater early decrease in depressive symptoms. While the early treatment response rate was low, certain patients did exhibit large decreases in symptoms. Within the sustained treatment responders (i.e. those entering into Phase 3), patients with greater 
increases in gamma $2 \mathrm{hrs}$ postinfusion had greater sustained decreases in depressive symptoms, and this increase was predictive of symptom decrease.

Higher baseline alpha power has been found to be associated with positive response to pharmacotherapy (Bruder et al., 2008; Tenke et al., 2011). In our study, higher baseline parietooccipital power was related to greater sustained (Phase 3) decrease in SI, possibly reflecting an association with vigilance regulation in MDD (Hegerl et al., 2012; Olbrich et al., 2016). In addition, greater ketamine-induced decreases in SI were found to be related to smaller decreases in alpha power. This was observed at early ( $2 \mathrm{hrs}$ and one day postinfusion) as well as sustained (Phase 2 and 3) time points. These findings may indicate that stable alpha power (i.e. less subject to alterations with ketamine or other pharmacotherapies) is related to greater decreases in SI. A recent study examining EEG changes in female MDD patients (suicide attempters, ideators, and non-suicidal) found a significant relationship between higher alpha power in ideators (vs. nonsuicidal, (Benschop et al., 2019), suggesting a relationship between alpha power and SI. Greater decreases in SI symptoms one day postinfusion were also related to higher baseline theta2 sgACC and rACC activity. Lee et al. (2017) found significantly higher theta power, with a strong relation between SI and fronto-central power, in a group of non-depressed individuals with high SSI. However, there is a general lack of research in this area, particularly with depressed participants.

Certain EEG measures were particularly predictive of the decrease in total MADRS and SI. Using only EEG measures, change in PF cordance was predictive of depressive symptom change at $2 \mathrm{hrs}$, and baseline theta $2 \mathrm{sgACC}$ was predictive of depressive symptom change at one day postinfusion. Sustained decrease in depressive symptoms were predicted by baseline rACC theta1 (Phase 2) and increase in frontal gamma power (Phase 3). With the addition of baseline 
MADRS and early changes in MADRS scores, only the model prediction change at one day postinfusion was altered to include both change at 2 hours postinfusion in addition to baseline theta 2 sgACC. These findings are particularly salient, as they suggest that baseline and early change in depressive symptoms alone are not as useful as baseline and early changes in EEG alone or in combination with early decreases in depressive symptoms with ketamine. This has been shown previously with various pharmacotherapies (Bares et al., 2015, 2017; de la Salle et al., 2020; Jaworska et al., 2019), and adds to the growing body of research suggesting that antidepressant treatment response outcomes may be optimally predicted by the combination of measures (e.g. clinical measures, EEG-derived, brain-derived neurotrophic factor (BDNF) serum and plasma levels, genetic polymorphisms). In many previous EEG-prediction studies, theta cordance and theta ACC power have shown the strongest relation to successful treatment prediction with varying treatments and study designs (Widge et al., 2019). The current study adds to and extends this finding, in that many of the strongest predictors of both early and sustained response to ketamine involved the theta band.

Using only EEG measures, decrease in SI at $2 \mathrm{hrs}$ post was predicted by $\Delta$ post alphaT power, and by baseline rACC theta 2 with $\Delta$ post alphaT power at one day postinfusion. Sustained decrease in SI (Phase 3) was most predicted by $\Delta$ post alphaT. However, with the addition of baseline and early change in MADRS SI, the EEG variables were replaced by the SI symptoms for most models; with the exception of $\Delta 1 \mathrm{~d}$ postinfusion, which resulted in a model combining change in SI symptoms at $2 \mathrm{hrs}$ with baseline rACC theta2. Baseline and early change in electrophysiological measures were not as reliable predictors as baseline and early change in SI symptoms. This is consistent with the current state of prediction of SI and attempts, with a metaanalysis of longitudinal research concluding that current predictors are only slightly better than 
chance (Franklin et al., 2017), as well as a lack of research for short-term risk prediction (Glenn \& Nock, 2014). Prediction of decreases in SI with ketamine or other antidepressant treatments may prove to be even more complex than the prediction of decreases in overall depressive symptoms, though neuroimaging-based predictors are currently being investigated, with some recent positive findings (Benschop et al., 2019; Just et al., 2017).

\subsection{Limitations}

Despites the strengths and novelties of this study, there are several limitations that warrant discussion. Regarding the clinical component of the study, there are limitations regarding the effectiveness of midazolam as a blinding agent (lack of dissociative side effects) and the open-label nature of the sustained treatment phases (may influence response rates). Additionally, the patient sample exhibited a high level of illness severity and treatment resistance as well as demonstrating a low antidepressant response to the first single infusion (27\% response in primary clinical study, $25 \%$ in this sub-sample), which may limit generalizability to other TRD samples.

Regarding electrophysiological limitations, there were no EEG recordings for one day postinfusion, which is typically when most patients show the greatest response, or at any points in Phase 2 and 3. Therefore, sustained changes in electrophysiological measures could not be examined. We also did not have a separate baseline session, which would be beneficial to have as a separate time point (aside from the testing days). Additionally, our resting EEG recordings were 3 minutes in duration, which does not allow for the assessment of vigilance. This may influence midline theta band activity (and consequently theta cordance and ACC-localized activity) and has also been shown to differ in antidepressant treatment responders, who display higher baseline brain arousal levels and greater decreases in vigilance with treatment (Schmidt et 
al., 2017). The measure of cordance has also been questioned in terms of its physiological interpretation (Bares et al., 2019). Finally, while EEG has excellent temporal resolution, it has inherent spatial resolution limitations, which is particularly important for our source-localized ACC data.

Finally, the overall sample size was limited to patients who were willing and able to participate in this electrophysiological component (over 5 hours in the EEG research lab) of a larger clinical trial. This did not allow for a binomial analysis of responders vs. non-responders and remitters vs. non-remitters at each time point, or the calculation of prediction measures such as sensitivity, specificity, positive and negative predictive values, or the identification of cut-off scores for future studies to form a priori hypotheses. While we did examine responders vs. nonresponders at one day and end of phase 2 in an exploratory manner, they must be interpreted with caution, though they are in line with previous findings with other antidepressant treatments. Despite the relatively small sample, it is a starting point for future larger trials seeking to determine electrophysiological based correlates and predictors of the antidepressant response to ketamine.

Future studies should aim to collect EEG measures at acute time points, as well as at key response time points (one day post) and throughout sustained treatment phases, including a final examination of sustained treatment responders to assess longer term electrophysiological changes in responders and non-responders. Gathering additional biomarkers (such as BDNF and blood-based metabolites) to be used in future prediction analyses would also be beneficial. Finally, the current work only examined frequency and regional activity changes, though measures such as coherence and connectivity would allow for the investigation of networks and 
potential cross-frequency changes with ketamine and their potential relationship to decrease in depressive symptoms.

\subsection{Conclusion}

This study examined acute ketamine-induces changes in electrophysiological measures and their relationship to early and sustained treatment response to sub-anesthetic ketamine in TRD MDD. To our knowledge, it is the first study to examine acute ketamine-induced changes in relation to repeated ketamine infusions. Overall, this study found regional- and frequencyspecific ketamine-induced changes in electrophysiological measures which were related to and predictive of decreases in depressive symptoms and SI. Early and sustained treatment responders also differed at baseline in surface level and source-localized theta. As certain patients may not respond immediately, being able to predict sustained response is of great importance, as the nonresponders may benefit from an alternative treatment regime (e.g. electroconvulsive therapy [ECT], repetitive transcranial magnetic stimulation [rTMS], deep brain stimulation [DBS]) offered prior to engaging in multiple sessions with ketamine. 
CHAPTER 3: Acute Subanesthetic Ketamine-Induced Effects on the Mismatch Negativity (MMN) and their Relationship to Early and Sustained Treatment Response in Major Depressive Disorder

\author{
Sara de la Salle ${ }^{* 1}$, Jennifer L. Phillips ${ }^{2}$, Pierre Blier ${ }^{2}$, Verner Knott ${ }^{1}$ \\ ${ }^{1}$ Clinical Electrophysiology and Cognitive Research Laboratory, University of Ottawa Institute \\ of Mental Health Research, Ottawa, ON, Canada \\ ${ }^{2}$ Mood Disorders Research Unit, University of Ottawa Institute of Mental Health Research, \\ Ottawa, ON, Canada
}

\title{
3.1 Abstract
}

Background: A sub-anesthetic dose of ketamine, a glutamate N-methyl-D-aspartate receptor (NMDA-R) antagonist, has been found to produce robust antidepressant effects in treatmentresistant major depressive disorder (MDD). The auditory mismatch negativity (MMN), a preattentive event-related potential, is strongly dependent on glutamatergic neurotransmission and is diminished by NMDA-R antagonists, and may be useful for characterizing neural mechanisms mediating the antidepressant response. This study examined the acute effects of ketamine and midazolam on the MMN and its relationship to early and sustained decreases in depressive symptoms.

Methods: Patients with treatment-resistant MDD $(\mathrm{N}=24)$, enrolled in a multi-phase clinical ketamine trial, received two infusions within the first double-blind crossover phase. Patients underwent two infusions (ketamine: $0.5 \mathrm{mg} / \mathrm{kg}$ or midazolam: $30 \mu \mathrm{g} / \mathrm{kg}$ ) approximately $1 \mathrm{week}$ apart. Three recordings were carried out per session (pre-, immediately post- and $2 \mathrm{hr}$ postinfusion). Peak MMN amplitude $(\mu \mathrm{V})$, latency (ms), theta event-related oscillations (ERO), theta phase locking factor (PLF), and source-localized MMN generator activity were assessed. The relationships between changes in MMN indices and early (Phase 1) and sustained (Phase 2,3) changes in depressive symptoms (Montgomery-Asberg Depression Rating Scale (MADRS) total score) were examined. 
Results: Ketamine reduced frontal MMN amplitudes, theta ERO immediately post- and $2 \mathrm{hr}$ postinfusion, and source-localized peak MMN frontal generator activity. Select baseline and ketamine-induced MMN decreases correlated and predicted greater early and sustained symptom decreases.

Conclusions: Acute pharmacological blockade of NMDARs reduced frontal MMN measures in patients with treatment-resistant MDD. Greater MMN reductions were related to and predictive of greater decreases in depressive symptoms. As the MMN does not require active attention, it may have the potential to act as a non-invasive biomarker for antidepressant response prediction to glutamatergic agents.

Keywords: Event-related oscillations; ketamine; Major depressive disorder; midazolam; mismatch negativity, phase-locking; theta; treatment response 


\subsection{Introduction}

\subsubsection{Role of Glutamate in Treatment-Resistant Major Depressive Disorder (MDD)}

Despite decades of research and a multitude of pharmacotherapeutic treatment options, questions regarding the pathophysiology of MDD remain. Many current treatments target the monoamine systems (serotonin, noradrenaline, dopamine), whose purported underactivity is responsible for depressive symptoms (Boku et al., 2018). Pharmacological agents which increase monoamines (i.e. tricyclic antidepressants [TCA], selective serotonin reuptake inhibitors [SSRI], serotonin-norepinephrine reuptake inhibitor [SNRI]) are primarily used for the treatment of major depressive disorder (MDD), though up to two thirds of treatment-seeking MDD patients do not achieve symptom relief and are considered to be treatment-resistant (Kennedy et al., 2001; Rush et al., 2006).

One avenue of investigation has been the glutamate system (Murrough et al., 2017). Alterations in concentrations and activity of glutamate, the primary excitatory neurotransmitter, and gamma-aminobutyric acid (GABA), the primary inhibitory neurotransmitter, have been observed within MDD populations, suggesting that excitatory/inhibitory signalling dysfunction may be related to MDD (Lener et al., 2017). Glutamate is also involved in synaptic plasticity regulation and plays a major role in mood, cognition, learning, and reward (Murrough et al., 2017). The N-methyl-D-aspartate receptor (NMDAR) antagonist ketamine has received considerable focus within human and animal research in the past two decades (Wei et al., 2020). While the exact mechanism had not been completely elucidated, the current state of knowledge has suggested that ketamine exerts its antidepressant effects through increased glutamate neurotransmission in the prefrontal cortex via NMDAR blockade and AMPA activation, leading 
to an increase in synaptic plasticity and neurotrophic signaling through downstream molecular cascades (Lener et al., 2017; Murrough et al., 2017).

Findings from independent research groups as well as multiple meta-analyses has consistently shown that a single sub-anesthetic dose of ketamine is able to produce rapid antidepressant effects in individuals with MDD (Caddy et al., 2015; Coyle \& Laws, 2015; Fond et al., 2014). However, these effects are transitory and typically peak within 24 hours, and depressive symptoms return within approximately one-week postinfusion (Kishimoto et al., 2016). Most placebo-controlled trials have used saline, however, the benzodiazepine midazolam used as an "active" placebo was found to be superior in terms of blind integrity (Wilkinson et al., 2019). Recent studies have shown that repeated ketamine infusions are able to prolong the effects (aan het Rot et al., 2010; Cusin et al., 2017; Murrough et al., 2013b; Shiroma et al., 2014; Singh et al., 2016; Vande Voort et al., 2016; Wilkinson et al., 2018b). Phillips et al. (2019) found that repeated infusions (thrice-weekly for 2 weeks) led to cumulative and sustained responses which were maintained in responders during maintenance infusions (once weekly, for a month).

\subsubsection{Mismatch Negativity (MMN), N-Methyl-D-Aspartate (NMDA) Receptor Function, and MDD}

Elucidating the manner in which ketamine alters underlying neural functioning may prove useful for understanding its antidepressant effects. The auditory mismatch negativity $(\mathrm{MMN})$ event-related potential is a commonly-studied early electrophysiological measure, which is modulated primarily by NMDA receptor signalling (Rosburg \& Kreitschmann-Andermahr, 2016), and has been suggested as a neurophysiological proxy of target engagement for NMDARassociated cortical plasticity (Kantrowitz et al., 2018). Recent work has suggested that the MMN, a frontocentral negative deflection peaking approximately 120-250 milliseconds (ms) post- 
stimulus, reflects a prediction-error based on regularity violation (as opposed to reflecting solely auditory "echoic" sensory memory, Näätänen, 1990), with the resulting MMN occurring only when a stimulus does not match the prediction based on the last stimulus that was presented (Winkler, 2007). The MMN is commonly elicited within paradigms of infrequent (deviant) and repeated (standard) stimuli and is quantified using a deviant-minus-standard difference calculation (Näätänen et al., 2007). Underlying the scalp-level MMN amplitudes are generators which include the bilateral superior temporal lobe, anterior cingulate gyrus, and right inferior temporal lobe (Waberski et al., 2001). The temporal MMN subcomponent is thought to be generated by pre-perceptual deviance detection, followed by involuntary switching of attention to the auditory deviance by the more frontal regions, producing the frontal MMN subcomponent (Rinne et al., 2000).

While not as frequently examined in MDD research, certain studies have found reduced MMNs (i.e. decreased amplitude, Qiao et al., 2013; Takei et al., 2009) as well as MMN increases (Bissonnette et al., 2020; He et al., 2010; Kähkönen et al., 2007; Restuccia et al., 2016), and longer latencies (Bissonnette et al., 2020; Qiao et al., 2013) in MDD. A recent study found a relationship between MMN amplitude and functional outcomes in MDD patients (Kim et al., 2020), suggesting that findings of reduced amplitudes may reflect pathological NMDAR functioning, which subsequently leads to impaired functioning. However, many factors may influence these findings (e.g. comorbid illnesses, illness severity, deviant type), and additional work is required.

MMN analyses primarily examine peak amplitude (microvolts $[\mu \mathrm{V}]$ ) and latency $(\mathrm{ms})$ in the time domain, however, the underlying phase-locked frequency-specific event-related oscillations (ERO) and measures of time-varying phase locking (phase locking factor, PLF) have 
been found to influence these measures. Measured in the time-frequency domain, ERO and PLF can provide sensitive and detailed descriptions of brain dynamics which underlie the MMN (Kaser et al., 2013). Increases in theta ERO and phase alignment for deviant stimuli are related to the frontal MMN subcomponent, while theta phase resetting without ERO modulation have been observed with the temporal component (Fuentemilla et al., 2008). Theta ERO have also been associated with attention and cognitive processes, and may serve an orienting function (Aftanas et al., 2001).

\subsubsection{Use of MMN Measures to Examine Ketamine's Antidepressant Effects}

NMDA antagonists have been shown to alter the MMN (decreased amplitude and increased latency) in both human (Rosburg \& Kreitschmann-Andermahr, 2016) and animal studies (Gil-Da-Costa et al., 2013). Within healthy human electroencephalography (EEG) and magnetoencephalography (MEG) studies, a bolus dose ranging from $0.15-30 \mathrm{mg} / \mathrm{kg}$ is typically administered prior to the continuous infusion, as the primary focus is the induction of psychotomimetic effects as a model for schizophrenia. One study used a comparatively lower dose $(0.30 \mathrm{mg} / \mathrm{kg}$ bolus with $0.213 \mathrm{mg} / \mathrm{kg})$ and found minimal psychotomimetic effects combined with no reductions in MMN (Oranje et al., 2000). As theta and PLF underlie MMN generation, NMDAR antagonists have also been found to alter both spontaneous (Hunt \& Kasicki, 2013) and auditory evoked theta activity (de la Salle et al., 2019 [Appendix II]; Lee et al., 2018).

The administration of the MMN requires no behavioural response or attention (a cognitive domain that is markedly altered with ketamine) as it is capturing pre-attentive detection processes. Combined with its reliance on NMDAR functioning and its purported relation to synaptic plasticity, it could serve as a feasible, non-invasive neurobiomarker for the 
antidepressant response prediction to glutamatergic agents. A recent examination within a sample of MDD patients receiving a sub-anesthetic dose of ketamine $(0.44 \mathrm{mg} / \mathrm{kg})$ found MMN increases 3-4 hours postinfusion (Sumner et al., 2020). The recording time was selected in order to assess improvements in depressive symptoms. The authors also found a significant relationship between greater forward connectivity modulation between the right primary auditory cortex and right inferior temporal cortex in response to deviant tones with greater decreases in symptoms one day postinfusion. This observed increase in MMN, though contrary to most human studies that have observed MMN reductions, was interpreted as a restoration of sensitivity to prediction errors found in MDD.

\subsubsection{The Current Study}

This ERP study was a component of a larger clinical trial (NCT01945047) aimed at enhancing and prolonging the antidepressant effect of ketamine through repeated infusions (Phillips et al., 2019). The objective was to examine the utility of MMN measures (amplitude, latency, ERO, PLF, source-localized peak MMN generator activity) in characterizing the acute electrocortical effects of midazolam and a sub-anesthetic dose of ketamine in TR MDD and to examine their relationship to changes in early and sustained depressive symptoms with repeated ketamine treatments.

A single deviant type (longer duration) that has been consistently found to be responsive to ketamine administration was employed (de la Salle et al., 2019 [Appendix II]; Gunduz-Bruce et al., 2012; Heekeren et al., 2008; Kreitschmann-Andermahr et al., 2001; Mathalon et al., 2014; Roser et al., 2011; Umbricht et al., 2000). Consistent with previous healthy control studies measuring acute changes in MMN, we expect to observe initial acute decreases in MMN, 
measures of theta (ERO and PLF), and frontal MMN generators, followed by a potential increase at $2 \mathrm{hrs}$ postinfusion with relations to early and sustained antidepressant treatment response.

\subsection{Methodology}

\subsubsection{Participants}

Refer to section 2.3.1 Participants

\subsubsection{Study Design}

Refer to section 2.3.2 Study Design

\subsubsection{Drug Administrations}

Refer to section 2.3.3 Drug Administrations

\subsubsection{EEG Acquisition}

Electrophysiological recordings were performed according to standard pharmaco-EEG procedures (Jobert et al., 2012) using a Brain Vision ${ }^{\circledR}$ Quickamp amplifier and Brain Vision Recorder® (Brain Products, Germany), with amplifier bandpass filters and sampling rate set to 0.1-100 Hz and $500 \mathrm{~Hz}$, respectively. Electrode impedances were maintained below $5 \mathrm{k} \Omega$. The montage included $32 \mathrm{Ag}^{+} / \mathrm{Ag}^{+} \mathrm{Cl}^{-}$passive electrodes (placed according to the $10-20$ international EEG system; Figure 3.1) and an electrode placed on the nose served as a reference. A midforehead electrode (AFz) served as the ground. Electro-oculographic activity was recorded from bipolar electrodes placed on the supra- and sub-orbital ridges of the right eye and on the external canthus of both eyes to record vertical (VEOG) and horizontal (HEOG). 


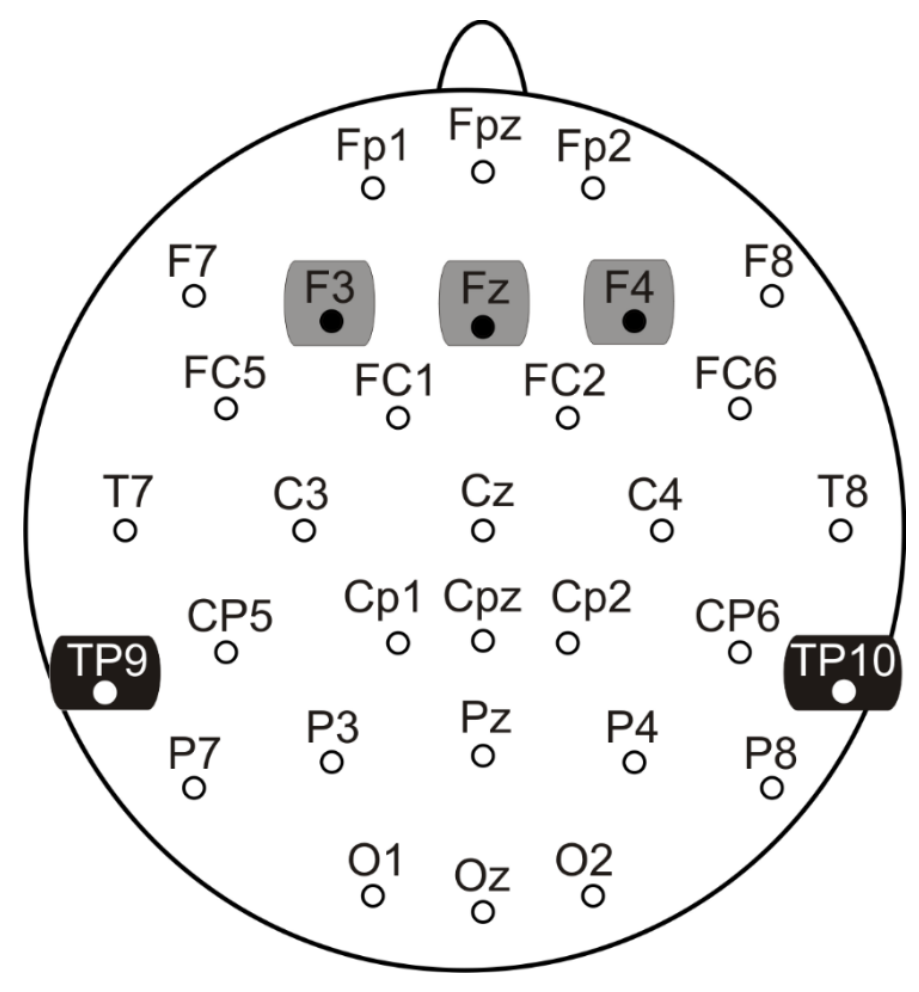

Figure 3.1. Electrode recording montage and electrode groupings (frontal=grey, mastoids=black) for analysis.

\subsubsection{Paradigm}

The MMN paradigm was administered following 3 minutes of eyes closed resting EEG.

Participants viewed a silent subtitled nature film (Madagascar, BBC, 2011). Auditory tonal stimuli (70 $\mathrm{dB}$ sound pressure level) were presented binaurally through noise-cancelling headphones. The sequence consisted of 1350 high probability $(p=0.9)$ standard stimuli (1000 $\mathrm{Hz}, 50 \mathrm{~ms}$ ), beginning with a series of 15 standard stimuli, and randomly intermixed with 150 low probability $(\mathrm{p}=0.1)$ longer duration deviant stimuli $(1000 \mathrm{~Hz}, 100 \mathrm{~ms})$, with a $500 \mathrm{~ms}$ fixed stimulus onset asynchrony. 


\subsubsection{MMN Analysis}

Brain Vision ${ }^{\circledR}$ Analyzer Version 2.2 software (Brain Products, Munich, Germany) was used for off-line ERP and wavelet processing. Raw data was re-referenced to the nose, bandpass filtered from 1-20Hz (24dB/oct; Duncan et al., 2009), ocular-corrected (Gratton et al., 1983), segmented (-100 to $400 \mathrm{~ms})$ separately for the standard and deviant stimuli, inspected for artifacts (voltages $\pm 75 \mu \mathrm{V}$, faulty channels, drift), and baseline corrected (relative to the prestimulus segment). Difference waveforms, derived by digital point-by-point subtraction of the standard stimuli from the deviant stimuli, were computed for each electrode site. The MMN was quantified $(\mu \mathrm{V})$ as the most negative peak ( $\pm 5 \mathrm{~ms})$ within $120-250 \mathrm{~ms}$ at the frontal electrodes (F3, Fz, F4) and as the most positive peak at the mastoid sites (TP9, TP10) in order to assess both MMN subcomponents. The obligatory N1 ERP elicited by the standard stimuli was defined as the most negative peak between $90-120 \mathrm{~ms}$ at the frontal midline electrode (Fz). Latencies (ms) for the MMN and the N1 were derived from the frontal midline site (Fz).

Event-related oscillations (EROs, $\mu \mathrm{V}$ ) were computed for averaged time-frequency epochs using a complex Morlet wavelet with a constant $\left(c=\sigma_{\mathrm{f}} / \mathrm{f}\right.$, where $\sigma_{\mathrm{f}}=$ the standard deviation of the centre frequency [f]) value of 5 over the range of $1-20 \mathrm{~Hz}, 1 \mathrm{~Hz}$ per frequency step (Hall et al., 2011). Phase-locking factor (PLF) values were computed using the Phase Locking Factor solution following wavelet transformations for each epoch, and subtracting the standard epochs from the deviants. PLF was calculated as 1 minus the circular variance of phases, with ranges from 0 (random distribution of phases) to 1 (perfect phase locking, TallonBaudry et al., 1996). Frontal (F3, F4, Fz) theta (4-8 Hz) ERO and PLF were exported for $\pm 50 \mathrm{~ms}$ around peak MMN amplitude. ERO values then ln-transformed to ensure that the data were normally distributed for statistical analysis. 


\subsubsection{Source-Localized Activity for MMN Generator Regions of Interest (ROIs)}

Source-localized current density $\left(\mathrm{A} / \mathrm{m}^{2}\right)$ was measured at peak MMN activity (based on ERP grand averages) from four previously defined ROIs representing four generators of the MMN (Heekeren et al., 2008; Waberski et al., 2001). This was performed with exact low resolution electromagnetic tomography (version 2081104; Pascual-Marqui et al., 2011). eLORETA is a weighted minimum non-linear inverse solution method applied to EEG recordings for computation of three dimensional distribution of electric cortical activity (PascualMarqui et al., 2011). The ROIs (Figure 3.2) were based on eLORETA-defined Brodmann Areas (BA) and included the four generators defined by Waberski et al. (2001): right superior temporal lobe (rSTL; BA38), left superior temporal lobe (ISTL; BA41), right inferior temporal lobe (rITL; BA37), and the anterior cingulate gyrus (ACC; BA32). Current density data from a single centroid representative voxel of each BA (the voxel closest to the center of the BA mass, which is an excellent representation of the corresponding BA) were extracted for further analysis. These regions were defined based on the Montreal Neurologic Institute average magnetic resonance imaging (MRI) brain (MNI 152) (Mazziotta et al., 2001) consisting of 6239 voxels $(5 \times 5 \times 5$ $\mathrm{mm}^{3} / \mathrm{voxel}$ ) and restricted to cortical gray matter/hippocampus. This method has been crossvalidated with functional and structural MRI, positron emission tomography (PET) and intracranial recordings (Mulert et al., 2004; Pizzagalli et al., 2004; Seeck et al., 1998; Vitacco et al., 2002; Worrell et al., 2000). 

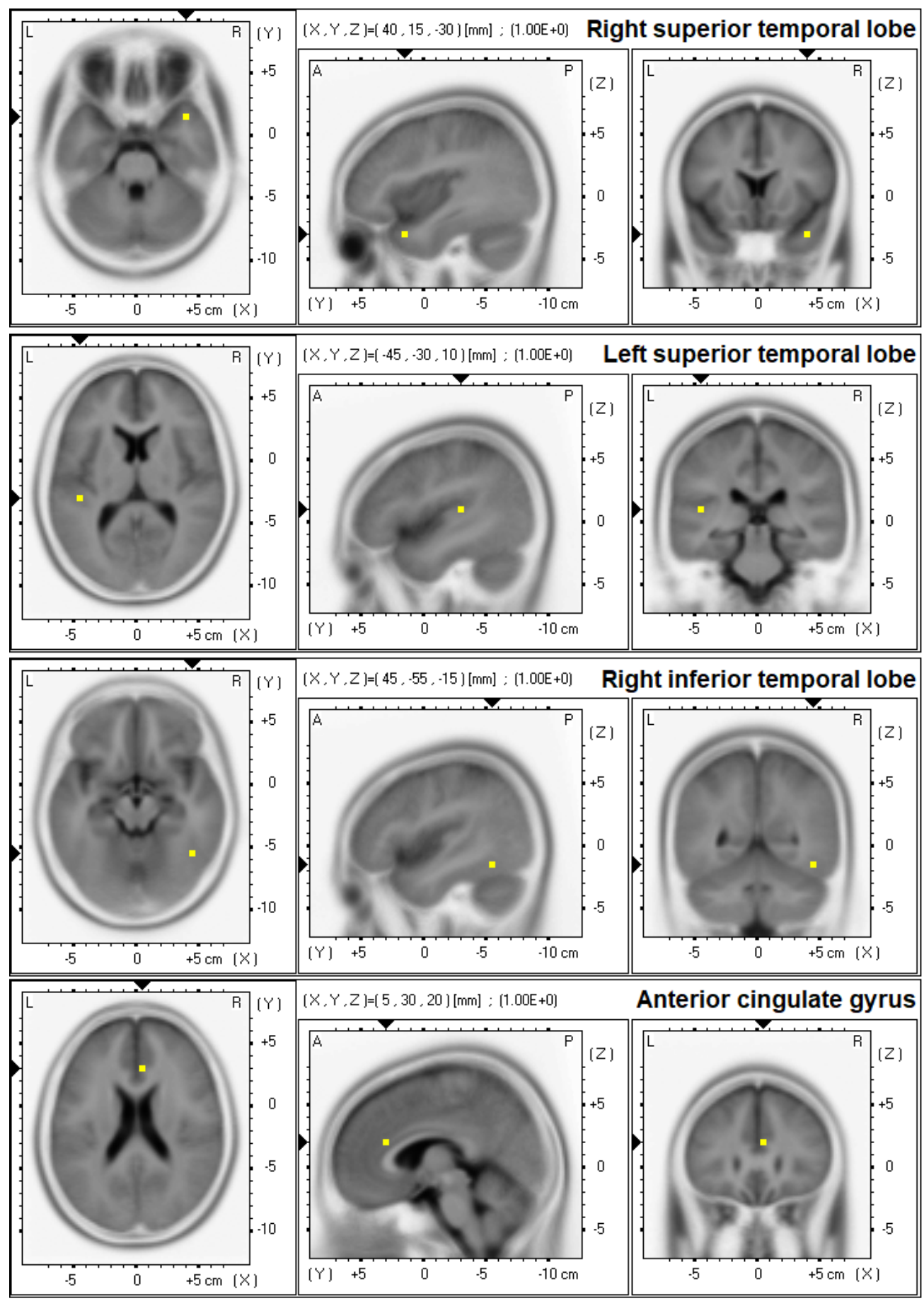

Figure 3.2. MMN temporal and frontal generator regions of interest (ROI) defined by (Waberski et al., 2001). 


\subsubsection{Clinical Outcomes and Dissociative Symptom Assessment}

Clinical outcome measures for the primary study are presented in Philips et al. (2019). For this smaller sample of patients who completed the EEG component of Phase 1, mean $( \pm$ S.D.) MADRS total scores are presented. Baseline measures were derived from the first session (regardless of drug). Comorbid disorders were assessed using the MINI at screening.

During Phase 1, the Brief Psychiatric Rating Scale-Positive Symptoms subscale (BPRSP, Grunebaum et al., 2018; Overall \& Gorham, 1962), which assesses conceptual disorganization, mannerisms and posturing, grandiosity, hostility, suspiciousness, hallucinatory behaviour, unusual thought content, and excitement, was administered at preinfusion, immediately postinfusion, $1 \mathrm{hr}$ and $2 \mathrm{hrs}$ postinfusion.

\subsubsection{Statistical Analysis}

Statistical analyses were carried out with the Statistical Package for Social Sciences (version 24, SPSS Inc., IBM, Armonk, N.Y.). Separate repeated measures analyses of variances (ANOVA) were used to assess changes in MMN indices of amplitude ( $\mu \mathrm{V})$, latency (ms), theta (4-8Hz) event-related oscillations (ERO, $\mu \mathrm{V})$, and theta phase locking factor (PLF).

For frontal MMN amplitude, theta ERO, and theta PLF analyses, within-group factors were drug condition (KET, ketamine; MID, midazolam), time (preinfusion, postinfusion, 2 hours postinfusion), and electrode site (F3 [left], F4 [right], Fz [midline]). ANOVAs for mastoid (temporal) MMNs were similar except for the electrode site factor (TP9 [left] and TP10 [right]). N1 amplitude and latency, as well as MMN latency did not contain a site factor as they were analyzed at Fz only; latency for temporal sites included TP9 and TP10.

The main effects and interactions of interest were drug condition and time; a priori drug condition $\mathrm{x}$ time interactions were performed for all measures. Natural logged (ln) source- 
localized CSD $\left(\mathrm{A} / \mathrm{m}^{2}\right)$ for each generator were analyzed separately with drug condition and time within-group factors. For all analyses, significant $(\mathrm{p}<0.05)$ Greenhouse-Geisser estimates were followed up with Bonferroni adjusted pairwise comparisons. Partial eta-squared $\left(\eta p^{2}\right)$ effect sizes are reported.

Relationships between MMN indices at baseline and their acute ketamine-induced changes (i.e. postinfusion - preinfusion, $\Delta$ postinfusion and $2 \mathrm{hrs}$ postinfusion - preinfusion, $\Delta 2 \mathrm{hrs}$ postinfusion) and early and sustained absolute change in MADRS (early change scores [i.e. $2 \mathrm{hrs}$ postinfusion - preinfusion, $\Delta 2 \mathrm{hrs}$; one day - preinfusion, $\Delta 1 \mathrm{~d}$ ], sustained change scores [i.e. Post-Phase 2 - Baseline, $\Delta \mathrm{Ph} 2$; Post-Phase 3 - Baseline, $\Delta \mathrm{Ph} 3$ ]) using Pearson correlations. Correlations between MMN indices and acute changes in BPRS-P ( $\triangle$ BPRS-P) symptoms were also performed.

Only electrophysiological variables (baseline and ketamine-induced changes) that were correlated with early and sustained change in MADRS were examined in terms of their predictive ability. Separate stepwise multiple regressions (Criteria: Probability-of-F-to-enter $<=$ .050 , Probability-of-F-to-remove $>=.100$ ) were performed with $\Delta 2 \mathrm{hrs}, \Delta 1 \mathrm{~d}, \Delta \mathrm{Ph} 2$, and $\Delta \mathrm{Ph} 3$ for MADRS total score as dependent variables. As early change in depressive symptoms can be predictive of later response, we also included early change in MADRS (i.e. $\Delta 2 \mathrm{hrs}$ and $\Delta 1 \mathrm{~d}$ ) in the regressions for sustained response $(\Delta \mathrm{Ph} 2, \Delta \mathrm{Ph} 3)$.

\subsection{Results}

\subsubsection{Demographics, Clinical outcome, and Dissociative Symptom Measures}

Refer to section 2.4.1 Demographics, clinical outcome, and dissociative symptom measures. 


\subsubsection{Changes in MMN Measures with Ketamine and Midazolam}

Frontal and temporal MMN amplitudes and latencies are shown in Table 3.1.

Topographical headmaps at peak MMN latencies are shown in Figure 3.3. Grand average

difference waveforms at the frontal electrode sites (F3, Fz, F4) and mastoid sites (TP9, TP10)

during each drug condition (KET, MID) and recording time (pre, post, 2hrs post) are displayed in

Figure 3.4.

Table 3.1. Mean $( \pm$ S.E.) peak MMN frontal and temporal subcomponent amplitudes $(\mu \mathrm{V})$ and latencies (ms) at each time point and drug condition.

\begin{tabular}{|c|c|c|c|c|c|c|c|c|c|}
\hline & \multicolumn{5}{|c|}{ Amplitudes $(\mu \mathrm{V})$} & \multicolumn{3}{|c|}{ Latency (ms) } \\
\hline & & F3 & F4 & $\mathbf{F z}$ & TP9 & TP10 & $\mathbf{F z}$ & TP9 & TP10 \\
\hline \multirow{3}{*}{ KET } & Pre & $\begin{array}{c}-1.89 \\
(.18)\end{array}$ & $\begin{array}{c}-2.14 \\
(.20)\end{array}$ & $\begin{array}{c}-2.22 \\
(.21)\end{array}$ & $\begin{array}{l}2.61 \\
(.29)\end{array}$ & $\begin{array}{l}2.15 \\
(.26)\end{array}$ & $\begin{array}{c}187.9 \\
(3.4)\end{array}$ & $\begin{array}{l}160.4 \\
(5.5)\end{array}$ & $\begin{array}{c}164.0 \\
(5.1)\end{array}$ \\
\hline & Post & $\begin{array}{c}-1.46 \\
(.13)\end{array}$ & $\begin{array}{c}-1.64 \\
(.14)\end{array}$ & $\begin{array}{c}-1.66 \\
(.14)\end{array}$ & $\begin{array}{l}2.04 \\
(.27)\end{array}$ & $\begin{array}{l}1.95 \\
(.23)\end{array}$ & $\begin{array}{c}190.2 \\
(5.0)\end{array}$ & $\begin{array}{l}165.2 \\
(3.8)\end{array}$ & $\begin{array}{c}168.3 \\
(4.7)\end{array}$ \\
\hline & $\begin{array}{l}\text { 2hrs } \\
\text { Post }\end{array}$ & $\begin{array}{c}-1.68 \\
(.15)\end{array}$ & $\begin{array}{r}-1.61 \\
(.17)\end{array}$ & $\begin{array}{r}-1.78 \\
(.18)\end{array}$ & $\begin{array}{l}2.13 \\
(.21)\end{array}$ & $\begin{array}{l}2.04 \\
(.18)\end{array}$ & $\begin{array}{c}185.6 \\
(3.9)\end{array}$ & $\begin{array}{l}165.3 \\
(4.1)\end{array}$ & $\begin{array}{l}165.6 \\
(4.8)\end{array}$ \\
\hline \multirow{3}{*}{ MID } & Pre & $\begin{array}{l}-1.77 \\
(.17)\end{array}$ & $\begin{array}{l}-1.87 \\
(.19)\end{array}$ & $\begin{array}{c}-1.94 \\
(.20)\end{array}$ & $\begin{array}{l}2.75 \\
(.26)\end{array}$ & $\begin{array}{l}2.34 \\
(.25)\end{array}$ & $\begin{array}{c}186.8 \\
(4.3)\end{array}$ & $\begin{array}{l}163.3 \\
(5.4)\end{array}$ & $\begin{array}{c}164.9 \\
(5.4)\end{array}$ \\
\hline & Post & $\begin{array}{l}-1.58 \\
(.17)\end{array}$ & $\begin{array}{c}-1.74 \\
(.19)\end{array}$ & $\begin{array}{l}-1.87 \\
(.19)\end{array}$ & $\begin{array}{l}1.96 \\
(.24)\end{array}$ & $\begin{array}{l}1.78 \\
(.23)\end{array}$ & $\begin{array}{c}186.2 \\
(5.6)\end{array}$ & $\begin{array}{l}160.0 \\
(4.7)\end{array}$ & $\begin{array}{l}157.4 \\
(4.6)\end{array}$ \\
\hline & $\begin{array}{l}\text { 2hrs } \\
\text { Post }\end{array}$ & $\begin{array}{l}-1.61 \\
(.19)\end{array}$ & $\begin{array}{c}-1.79 \\
(.18)\end{array}$ & $\begin{array}{l}-1.82 \\
(.19)\end{array}$ & $\begin{array}{l}1.97 \\
(.26)\end{array}$ & $\begin{array}{l}2.02 \\
(.19)\end{array}$ & $\begin{array}{c}184.9 \\
(5.1)\end{array}$ & $\begin{array}{l}164.5 \\
(4.9)\end{array}$ & $\begin{array}{l}167.9 \\
(4.7)\end{array}$ \\
\hline
\end{tabular}

KET, Ketamine; MID, Midazolam; Post, immediately postinfusion; Pre, preinfusion; 2hrs post, $2 \mathrm{hrs}$ postinfusion

\subsubsection{Frontal Amplitude and Latency}

rmANOVAs indicated main effects of time $\left(\mathrm{F}[2,44]=7.29, p=.002, \eta \mathrm{p}^{2}=.25\right)$,

electrode $\mathrm{F}\left([1.6,34.4]=6.01, p=.01, \eta \mathrm{p}^{2}=.22\right)$, and main interaction effect drug $\mathrm{x}$ time $\mathrm{x}$ electrode $\mathrm{F}\left([4,88]=2.55, p=.04, \eta \mathrm{p}^{2}=.10\right)$. MMN amplitudes were reduced from pre- to postinfusion $(p=.007)$ and were larger at Fz compared to F3 $(p=.0001)$. Within the drug $\mathrm{x}$ time $\mathrm{x}$ electrode interaction, MMN amplitudes were reduced from pre- to postinfusion for $\mathrm{F} 3$ ( $p=$ $.003), \mathrm{F} 4(p=.01)$, and $\mathrm{Fz}(p=.003)$ and from pre- to $2 \mathrm{hrs}$ postinfusion for $\mathrm{F} 4(p=.03)$ and $\mathrm{Fz}$ 
$(p=.04)$ in the ketamine condition. No significant changes were observed in the midazolam condition.

No main effects for drug condition $\left(\mathrm{F}[1,22]=.38, p=.54, \eta \mathrm{p}^{2}=.02\right)$, time $(\mathrm{F}[2,44]=.92$, $\left.p=.41, \eta \mathrm{p}^{2}=.04\right)$, or drug $\mathrm{x}$ time $\left(\mathrm{F}[2,44]=.16, p=.85, \eta \mathrm{p}^{2}=.007\right)$ were observed for the analysis of frontal midline latency.

\subsubsection{Temporal Amplitude and Latency}

rmANOVAs indicated main effects of time $\left(\mathrm{F}[2,44]=5.23, p=.009, \eta p^{2}=.19\right)$ and electrode $\mathrm{F}\left([1,22]=5.57, p=.03, \eta p^{2}=.20\right)$, and main interaction effect of time $\mathrm{x}$ electrode $\mathrm{F}\left([2,44]=8.03, p=.001, \eta \mathrm{p}^{2}=.27\right)$. Temporal MMN amplitudes were reduced from pre- to postinfusion $(p=.03)$ and were larger at TP9 compared to TP10 $(p=.03)$. Within the time $\mathrm{x}$ electrode interaction, amplitudes were larger at TP9 compared to TP10 at preinfusion $(p=.001)$ and were reduced immediately post- $(p=.008)$ and $2 \mathrm{hrs-post} \mathrm{infusion}(p=.01)$ as compared to preinfusion for TP9 only. Within a planned drug $\mathrm{x}$ time $\mathrm{x}$ electrode interaction $\mathrm{F}([2,44]=.46, p$ $\left.=.64, \eta \mathrm{p}^{2}=.02\right)$, amplitudes were reduced from pre- to postinfusion for TP9 $(p=.003)$ and TP10 $(p=.005)$, and from pre- to 2 hrs-postinfusion $(p=.0001)$ for TP9 only in the midazolam condition. No significant decreases were found in the ketamine drug condition.

No main effects for drug condition $\left(\mathrm{F}[1,22]=.22, p=.64, \eta \mathrm{p}^{2}=.01\right)$, time $(\mathrm{F}[2,44]=.61$, $\left.p=.41, \eta \mathrm{p}^{2}=.02\right)$, or drug $\mathrm{x}$ time $\left(\mathrm{F}[2,44]=.81, p=.45, \eta \mathrm{p}^{2}=.04\right)$ were observed for the analysis of temporal latency. 


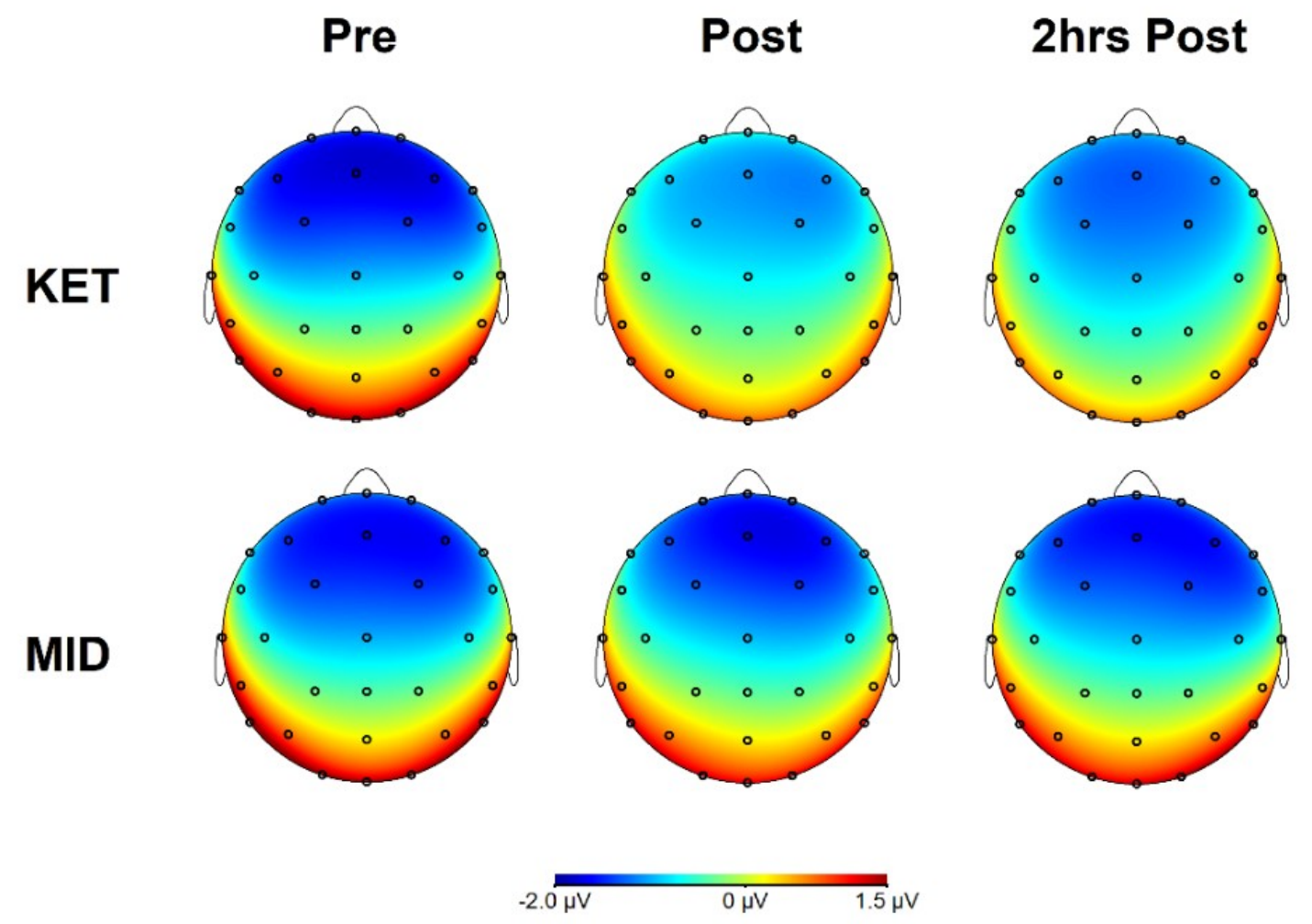

Figure 3.3. Peak MMN topographic maps at each time point and drug condition. 
KET

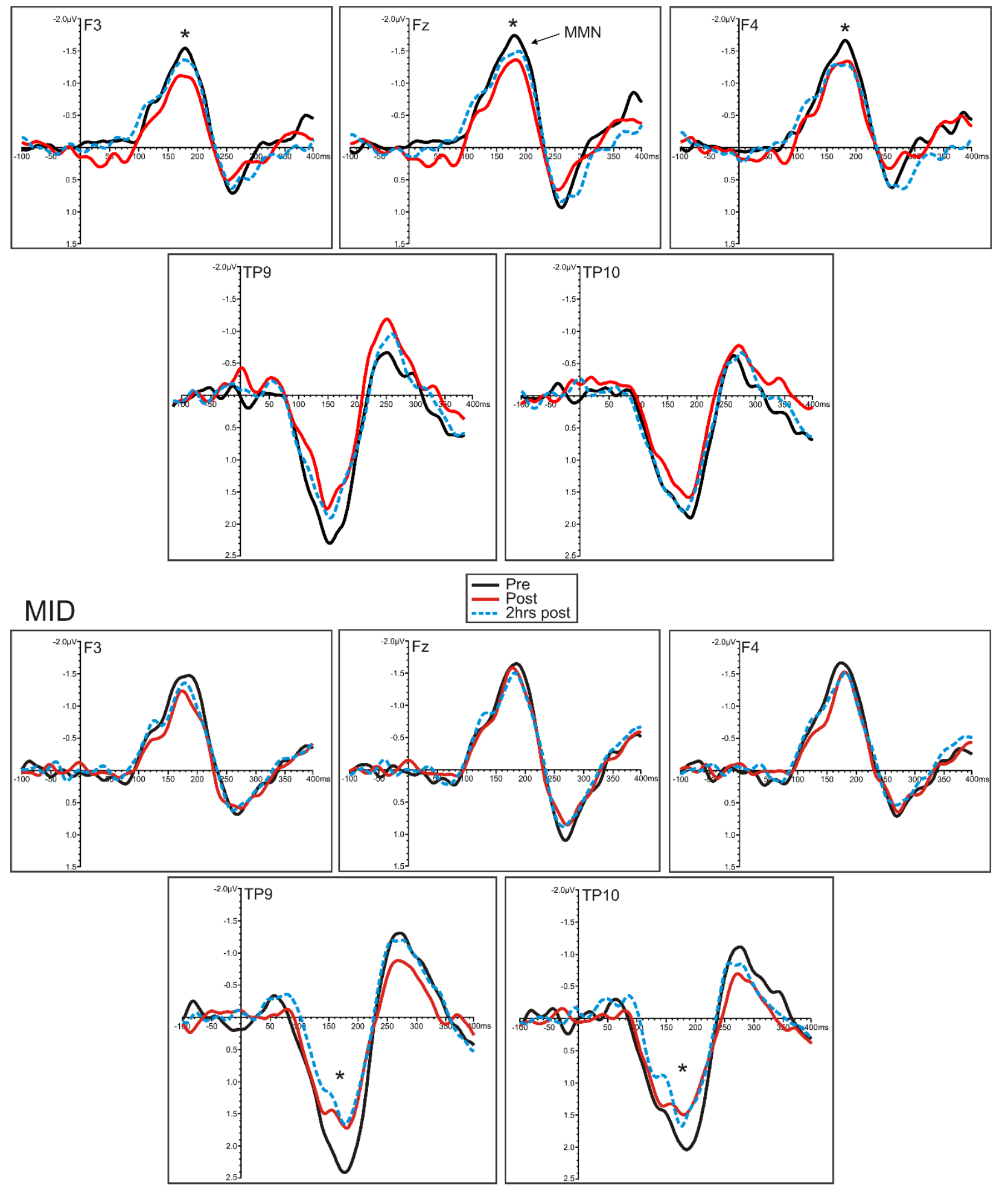

Figure 3.4. Grand averaged frontal (F3, left; Fz, midline; F4, right) and temporal (TP9, left; TP10, right) MMN difference waveforms at each time point and drug condition. $*=$ time comparison, $\mathrm{p}<.05$ 


\subsubsection{N1 Amplitude and Latency}

\section{N1 amplitude}

No main effects for drug condition $\left(\mathrm{F}[1,22]=3.46, p=.08, \eta p^{2}=.14\right)$, time $(\mathrm{F}[2,44]=$ $\left.2.23, p=.12, \eta p^{2}=.09\right)$, or drug $\mathrm{x}$ time $\left(\mathrm{F}[2,44]=.59, p=.56, \eta \mathrm{p}^{2}=.03\right)$ were observed for the analysis of N1 amplitude.

\section{N1 latency}

A main effects of time $\left(\mathrm{F}[2,44]=4.04, p=.03, \eta \mathrm{p}^{2}=.16\right)$ and drug $\mathrm{x}$ time $(\mathrm{F}[2,44]=$ $3.46, p=.04, \eta p^{2}=.14$ ) were found for $\mathrm{N} 1$ latency. Longer latencies were found at postinfusion as compared to pre- $(p=.005)$ and 2 hrs-postinfusion $(p=.01)$ within the midazolam condition only.

N1 amplitudes and latencies are shown in Table 3.2, and topographical headmaps are shown in Figure 5.

Table 3.2. Mean $( \pm$ S.E. $)$ peak $N 1$ amplitudes $(\mu \mathrm{V})$ and latencies $(\mathrm{ms})$ at each time point and drug condition.

\begin{tabular}{|c|c|c|c|}
\hline & & Amplitude $(\mu V)$ & Latency (ms) \\
\hline \multirow{3}{*}{ KET } & Pre & $-.23(.16)$ & $104.0(2.7)$ \\
\hline & Post & $-.27(.13)$ & $104.9(2.9)$ \\
\hline & 2hrs Post & $-.30(.13)$ & $104.7(3.1)$ \\
\hline \multirow{3}{*}{ MID } & Pre & $-.18(.14)$ & $103.0(2.5)$ \\
\hline & Post & $-.20(.14)$ & $113.3(3.4)$ \\
\hline & 2hrs Post & $-.24(.14)$ & $107.4(2.8)$ \\
\hline
\end{tabular}

KET, Ketamine; MID, Midazolam; Post, immediately postinfusion; Pre, preinfusion; $2 \mathrm{hrs}$ post, $2 \mathrm{hrs}$ postinfusion 

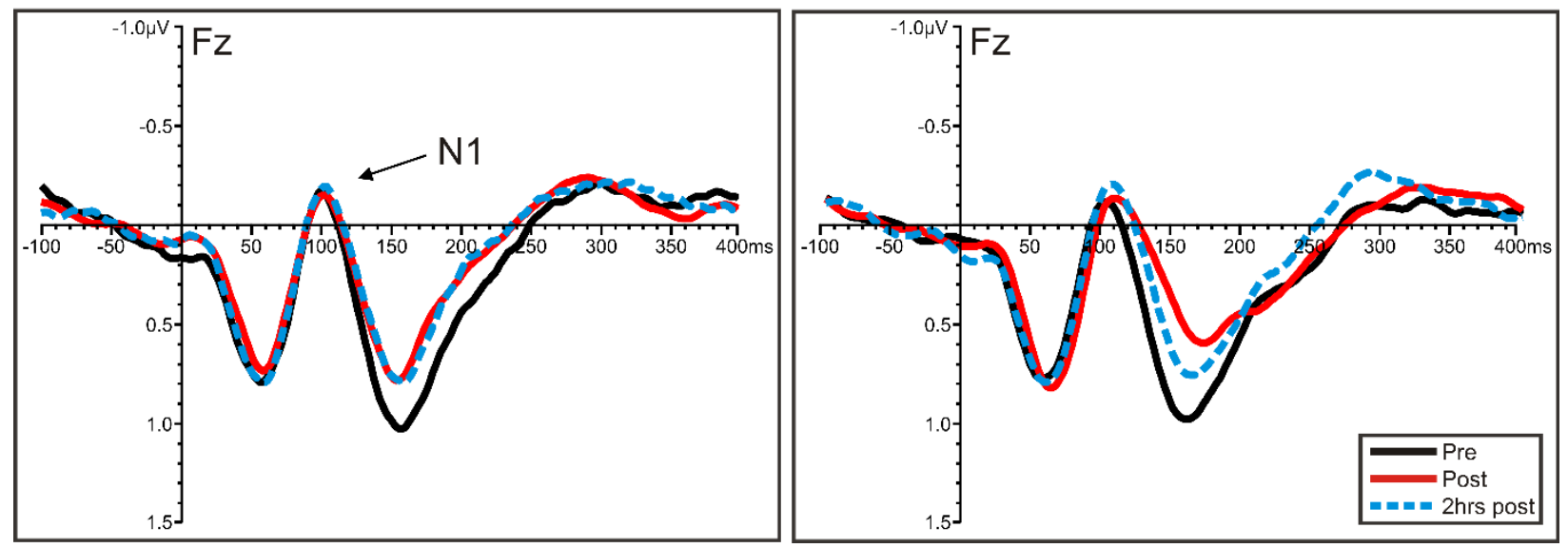

Figure 2.5. Frontal midline $(\mathrm{Fz})$ grand averaged $\mathrm{N} 1$ waveforms at each time point and drug condition. $*=$ time comparison, $\mathrm{p}<.05$

\subsubsection{Theta Event-Related Oscillations (ERO) and Phase Locking Factor (PLF)}

rmANOVAs indicated main effects of time $\left(\mathrm{F}[2,44]=6.58, p=.003, \eta \mathrm{p}^{2}=.23\right)$,

electrode $\left.\mathrm{F}[1.5,33.3]=19.08, p=.0001, \eta p^{2}=.46\right)$, and main interaction effect of drug $\mathrm{x}$ time $\left(\mathrm{F}(2,44]=3.47, p=.04, \eta p^{2}=.14\right)$. Theta ERO were reduced from pre- to postinfusion $(p=$ $.004)$ and 2 hrs postinfusion $(p=.02)$, and ERO values were larger for Fz vs. F3 ( $p=.0001)$. Within the drug $\mathrm{x}$ time interaction, ERO values were reduced from pre- to postinfusion $(p=$ $.006)$ and $2 \mathrm{hrs}$ postinfusion $(p=.01)$ in the ketamine drug condition only. No significant changes were observed in the midazolam condition.

No main effects for drug condition $\left(\mathrm{F}[1,22]=.75, p=.40, \eta \mathrm{p}^{2}=.03\right)$, time $(\mathrm{F}[2,44]=$ $\left.1.93, p=.16, \eta p^{2}=.08\right)$, or drug x time $\left(\mathrm{F}[2,44]=.58, p=.56, \eta p^{2}=.03\right)$ were observed for the analysis of theta PLF.

Theta ERO and PLF time-frequency plots are shown in Figure 3.6 and values are shown

\section{in Table 3.3.}




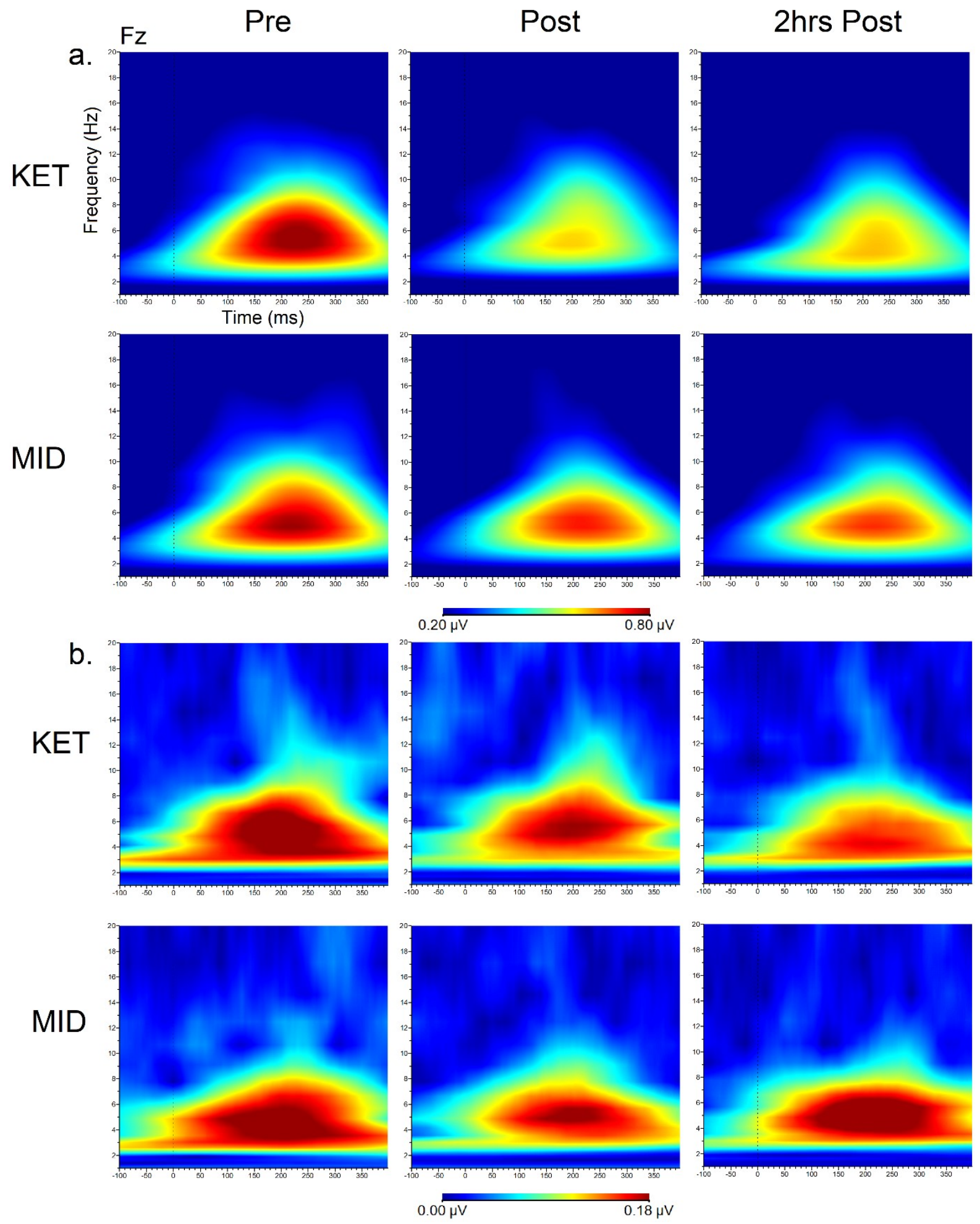

Figure 3.6. Grand averaged time frequency plots showing theta (a) event-related oscillations $(\mu \mathrm{V})$ and (b) phase-locking factor at the frontal midline (Fz) site at each time point and drug condition. 
Table 3.3. Mean ( \pm S.E.) peak MMN frontal event-related oscillations $(\mu \mathrm{V})$ and phase locking factor (PLF) values at each time point and drug condition.

\begin{tabular}{|l|l|c|c|c|}
\cline { 3 - 5 } \multicolumn{2}{c|}{} & \multicolumn{3}{|c|}{ ERO $(\boldsymbol{\mu} V)$} \\
\cline { 3 - 5 } \multicolumn{1}{c|}{} & F3 & F4 & Fz \\
\hline \multirow{3}{*}{ KET } & Pre & $.58(.06)$ & $.62(.06)$ & $.74(.08)$ \\
\cline { 2 - 5 } & Post & $.49(.06)$ & $.52(.06)$ & $.63(.09)$ \\
\cline { 2 - 5 } & 2hrs Post & $.50(.06)$ & $.52(.05)$ & $.62(.07)$ \\
\hline \multirow{3}{*}{ MID } & Pre & $.58(.08)$ & $.60(.06)$ & $.71(.09)$ \\
\cline { 2 - 5 } & Post & $.49(.05)$ & $.49(.05)$ & $.61(.06)$ \\
\cline { 2 - 5 } & 2hrs Post & $.48(.06)$ & $.52(.06)$ & $.60(.07)$ \\
\hline
\end{tabular}

\begin{tabular}{|c|c|c|}
\hline \multicolumn{3}{|c|}{ PLF } \\
\hline F3 & F4 & Fz \\
\hline $.17(.02)$ & $.18(.02)$ & $.18(.02)$ \\
\hline $.16(.01)$ & $.16(.02)$ & $.18(.02)$ \\
\hline $.15(.02)$ & $.18(.02)$ & $.17(.02)$ \\
\hline $.17(.02)$ & $.18(.02)$ & $.18(.02)$ \\
\hline $.14(.02)$ & $.15(.02)$ & $.15(.02)$ \\
\hline $.16(.02)$ & $.16(.02)$ & $.17(.02)$ \\
\hline
\end{tabular}

KET, Ketamine; ERO, event-related oscillations; MID, Midazolam; PLF, phase-locking factor; Post, immediately postinfusion; Pre, preinfusion; $2 \mathrm{hrs}$ post, $2 \mathrm{hrs}$ postinfusion

\subsubsection{Source-localized MMN Peak ROIs}

No main effects for drug condition, time, or drug $\mathrm{x}$ time were observed for the three temporal generators (rSTL, 1STL, rITL). rmANOVAs indicated a main interaction effect of drug $\mathrm{x}$ time $\left(\mathrm{F}(2,44]=5.30, p=.009, \eta \mathrm{p}^{2}=.19\right)$ for the ACC. CSD was reduced from pre- to postinfusion $(p=.02)$ in the ketamine condition only. Ln-logged CSD values are shown in Table 3.4.

Table 3.4. Mean ( \pm S.E.) ln-transformed source-localized CSD values $\left(\mathrm{A} / \mathrm{m}^{2}\right)$ of peak $\mathrm{MMN}$ generator activity

\begin{tabular}{|c|c|c|c|c|c|}
\hline & & \multicolumn{4}{|c|}{ Peak MMN Generator CSD } \\
\hline & & rSTL & ISTL & rITL & $\mathbf{A C C}$ \\
\hline \multirow{3}{*}{ KET } & Pre & $-7.84(.22)$ & $-9.54(.14)$ & $-8.96(.13)$ & $-9.26(.20)$ \\
\hline & Post & $-7.95(.23)$ & $-9.34(.19)$ & $-9.24(.18)$ & $-9.80(.17)$ \\
\hline & 2hrs Post & $-8.10(.23)$ & $-9.40(.16)$ & $-9.26(.19)$ & $-9.77(.18)$ \\
\hline \multirow{3}{*}{ MID } & Pre & $-8.00(.18)$ & $-9.53(.16)$ & $-9.14(.16)$ & $-9.47(.18)$ \\
\hline & Post & $-8.06(.21)$ & $-9.26(.13)$ & $-9.23(.18)$ & $-9.36(.17)$ \\
\hline & 2hrs Post & $-8.22(.21)$ & $-9.55(.18)$ & $-9.46(.19)$ & $-9.59(.20)$ \\
\hline
\end{tabular}

ACC, anterior cingulate cortex; CSD, current source density; KET, Ketamine; ISTL, left superior temporal lobe; MID, Midazolam; Post, immediately postinfusion; Pre, preinfusion; $2 \mathrm{hrs} \mathrm{post,} 2 \mathrm{hrs}$ postinfusion; rITL, right inferior temporal lobe; rSTL, right superior temporal lobe 


\subsubsection{Relationship between Ketamine-induced Changes in MMN and in BPRS-P}

Positive correlations between $\triangle \mathrm{BPRS}$ and baseline theta Fz ERO $(\mathrm{r}=.44, p=.04, \mathrm{~N}=$ 23), and baseline F4 theta PLF $(r=.44, p=.04, \mathrm{~N}=23)$ were found.

\subsubsection{Relationship between Baseline and Ketamine-induced Changes and MADRS $\Delta 2 \mathrm{hrs}$,} $\Delta 1 \mathrm{~d}, \Delta \mathrm{Ph} 2(\mathrm{~N}=23)$ and $\Delta \mathrm{Ph} 3(\mathrm{~N}=13)$ MADRS

Significant correlations are listed in Table 3.5 and scatterplots are displayed in Figure 3.7. Relationships were found between baseline and ketamine-induced MMN changes with both early and sustained treatment response.

Table 3.5. Significant correlations $(r, p)$ between baseline $M M N$ measures with change in Montgomery-Åsberg Depression Rating Scale (MADRS) at 2 hours ( $\Delta 2 \mathrm{hrs})$ and one day ( $\Delta 1 \mathrm{~d}$ ) postinfusion, and change from baseline to end of phase $2(\Delta \mathrm{Ph} 2)$ and end of phase $3(\Delta \mathrm{Ph} 3)$.

\begin{tabular}{|c|c|c|c|c|c|}
\hline & & \multicolumn{4}{|c|}{ MADRS } \\
\hline \multicolumn{2}{|c|}{ MMN Measure } & $\Delta 2 \mathrm{hrs}(\mathrm{N}=23)$ & $\Delta 1 d(N=23)$ & $\Delta \mathrm{Ph} 2(\mathrm{~N}=23)$ & $\Delta \operatorname{Ph} 3(N=13)$ \\
\hline \multirow{2}{*}{ Baseline } & rITL CSD & $\mathrm{r}=.59, \mathrm{p}=.003$ & & & $\mathrm{r}=-.81, \mathrm{p}=.001$ \\
\hline & F3 PLF & & $\mathrm{r}=.53, \mathrm{p}=.009$ & $r=.49, p=.02$ & \\
\hline \multirow{3}{*}{$\Delta$ postinfusion } & $\mathrm{F} 3 \mu \mathrm{V}$ & $\mathrm{r}=-.57, \mathrm{p}=.005$ & $\mathrm{r}=-.59, \mathrm{p}=.003$ & $\mathrm{r}=-.57, \mathrm{p}=.006$ & \\
\hline & $\mathrm{F} 4 \mu \mathrm{V}$ & $\mathrm{r}=-.55, \mathrm{p}=.007$ & $\mathrm{r}=-.55, \mathrm{p}=.007$ & & \\
\hline & $\mathrm{Fz} \mu \mathrm{V}$ & $r=-.56, p=.006$ & $\mathrm{r}=-.50, \mathrm{p}=.02$ & & \\
\hline \multirow{3}{*}{$\Delta 2 \mathrm{hrs}$ post } & $\mathrm{Fz} \mu \mathrm{V}$ & $\mathrm{r}=-.47, \mathrm{p}=.03$ & $\mathrm{r}=-.45, \mathrm{p}=.03$ & & \\
\hline & $\mathrm{F} 4 \mu \mathrm{V}$ & & $\mathrm{r}=-.44, \mathrm{p}=.04$ & & \\
\hline & Theta ERO & $\mathrm{r}=.51, \mathrm{p}=.01$ & $\mathrm{r}=.51, \mathrm{p}=.01$ & & \\
\hline
\end{tabular}

ACC, anterior cingulate cortex; CSD, current source density; ERO, event-related oscillations; PLF, phase-locking factor 

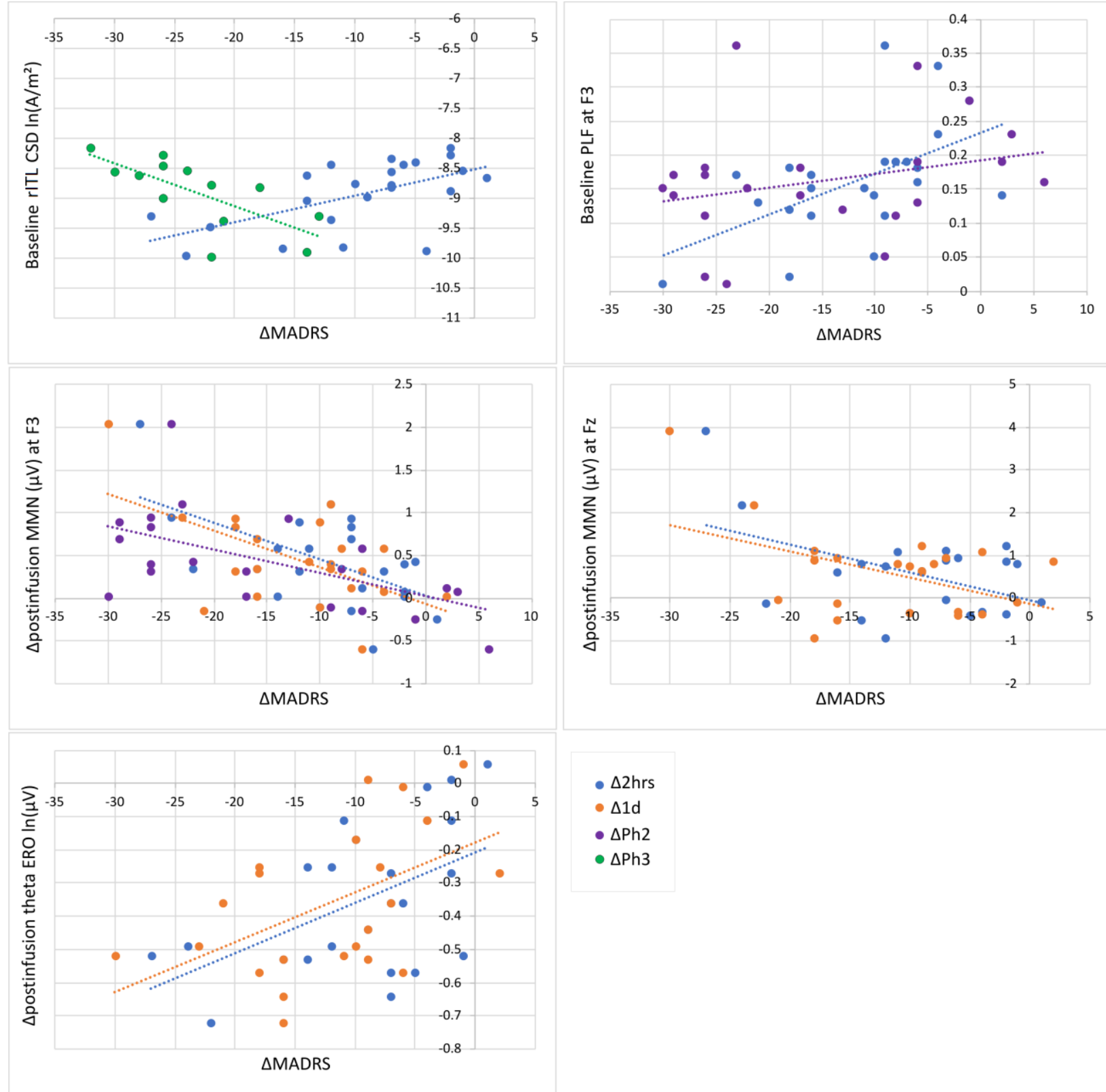

Figure 3.7. Scatterplots of significant correlations between baseline and ketamine-induced changes in MMN measures with change in Montgomery-Åsberg Depression Rating Scale (MADRS) at 2 hours ( $\triangle 2 \mathrm{hrs}$ ) and one day ( $\Delta$ 1d) postinfusion, and change from baseline to end of phase $2(\Delta \mathrm{Ph} 2)$ and end of phase $3(\Delta \mathrm{Ph} 3)$. 


\subsubsection{Prediction of Acute and Sustained Response to Ketamine}

Significant stepwise multiple regressions predicting change in MADRS at each time point were found. The model parameters (unstandardized Beta coefficient $[B]$, standard error [S.E.)], t-statistic $[t]$ and significance $[p]$ ) for all regression analyses are reported in Table 3.6.

With the addition of baseline and early change in MADRS, the only model that was changed was for $\Delta 1 \mathrm{~d}$ MADRS $\left(\mathrm{F}[2,22]=14.25, p=.0001, \mathrm{R}^{2}=.59\right)$, which included $\Delta 2 \mathrm{hrs}$ MADRS $(\mathrm{B}=.59$ $\pm .15, \mathrm{t}=3.84, p=.001)$ and F3 PLF baseline $(\mathrm{B}=35.2 \pm .13 .5, \mathrm{t}=2.60, p=.02)$

Table 3.6. Model parameters for regression analyses

\begin{tabular}{|c|c|c|c|c|c|c|}
\hline & Model & Independent variables & $B$ & S.E. & $\mathbf{t}$ & $p$ \\
\hline$\Delta 2 \mathrm{hrs}$ & $\mathrm{F}[2,22]=8.31$ & $\Delta$ postinfusion MMN (F3) $\mu \mathrm{V}$ & -6.13 & 2.30 & -2.66 & .02 \\
\hline & $p=.02, \mathrm{R}^{2}=.4 \mathrm{~J}$ & $\Delta$ postinfusion ERO & 12.67 & 5.77 & 2.20 & .04 \\
\hline$\Delta 1 \mathrm{~d}$ & $\mathrm{~F}[2,22]=11.58$ & $\Delta$ postinfusion MMN (F3) $\mu \mathrm{V}$ & -6.99 & 2.12 & -3.30 & .004 \\
\hline & & Baseline left PLF & 40.60 & 14.04 & 2.89 & .009 \\
\hline$\Delta \mathrm{Ph} 2$ & $\begin{array}{l}\mathrm{F}[1,21]=6.17 \\
p=.02, \mathrm{R}^{2}=.49\end{array}$ & Baseline left PLF & 67.93 & 27.36 & 2.48 & .02 \\
\hline$\Delta \mathbf{P h 3}$ & $\begin{array}{c}\mathrm{F}[1,12]=21.58 \\
p=.001, \mathrm{R}^{2}=.66\end{array}$ & Baseline rITL CSD & -9.27 & 1.99 & -4.65 & .001 \\
\hline
\end{tabular}

B, Beta coefficient; S.E., standard error

\subsection{Discussion}

This ERP study examined acute changes in MMN-derived measures (amplitude, latency, ERO, PLF, peak MMN generator activity) with a sub-anesthetic dose of ketamine in comparison to the active placebo, midazolam. Baseline and ketamine-induced MMN changes were then examined in relation to early (Phase 1) and sustained (Phases 2,3) decreases in depressive symptoms with ketamine. 
Within the randomized, double-blind, crossover phase of the study, decreases in MMN amplitude, theta ERO, and the ACC frontal MMN generator were observed with ketamine, while midazolam decreased temporal MMN amplitudes and increased postinfusion N1 latency. These findings are line with previous human studies that have observed acute decreases in MMN measures with ketamine (Rosburg \& Kreitschmann-Andermahr, 2016), as well as decreases in the temporal MMN subcomponent and increases N1 latency with benzodiazepines (Rosburg et al., 2004). Neither the N1 amplitude nor the temporal MMN amplitudes were reduced with ketamine, however, decreases in theta ERO ( $\Delta$ postinfusion and $\Delta 2 \mathrm{hrs})$ and the frontal MMN generator ( $\Delta$ postinfusion only) were observed. Together, these findings suggest that the decreased in scalp-level frontal MMN amplitude values, occurring with decreases in the frontal generator and theta oscillations, are due to ketamine-specific alterations the neuronal processes which underlie the auditory MMN. There were no drug-induced changes in PLF, which may be due to the latency range extracted (based on frontal midline), as theta PLF measures have a stronger relation to the temporal subcomponent.

Contrary to our hypothesis and to Sumner et al. (2020), we did not observe increases in MMN measures at our later recording time (2hrs postinfusion). Major differences that could account for these findings are the time of recording, MMN task, the participant sample, and response rates. The time of recording was 3-4 hours postinfusion, vs. immediately postinfusion and 2 hours postinfusion recorded in the current study. It is possible that MMN increases above the initial preinfusion level following the initial physiological decrease with NMDAR antagonism. The authors employed a roving auditory oddball task, which may capture different aspects of the MMN-indexed ketamine response. Additionally, the participants had less severe treatment-resistance and exhibited much greater antidepressant responses at both $3 \mathrm{hrs}$ and one 
day post as compared to our study. This may indicate a greater responsiveness for restoration of sensitivity to prediction errors impaired in MDD. With larger samples, comparisons could be made regarding depression severity level and subsequent increases vs. sustained decreases following sub-anesthetic ketamine infusions.

Greater increase in BPRS-P symptoms from pre- to immediately postinfusion was associated with higher baseline frontal midline theta ERO and right frontal PLF. In our previous related work in healthy controls (de la Salle et al., 2019 [Appendix II]), a similar relationship was found between increase in derealization symptoms and baseline midline frontal PLF. These findings suggest that individuals with higher event-related theta oscillations and phase synchronization are more susceptible to the 'positive' symptoms induced by ketamine. As theta ERO have been associated with passive attention and stimulus orientation (Karakaş, 2020), these individuals may be more sensitive to the psychotomimetic effects of ketamine.

Early decreases in depressive symptoms at $2 \mathrm{hrs}$ and one day postinfusion were related to decreases in MMN amplitudes and theta ERO, as well as lower baseline rITL peak MMN generator activity ( $\Delta 2 \mathrm{hrs})$ and higher left frontal baseline PLF values $(\Delta 1 \mathrm{~d})$. Sustained decreases in depressive symptoms were related to decreases in left frontal MMN amplitude and lower left frontal baseline PLF $(\triangle \mathrm{Ph} 2)$, and higher baseline rITL peak MMN generator activity $(\Delta \mathrm{Ph} 3)$. These findings indicate that a better early response to a single sub-anesthetic ketamine administration is related to greater decreases in MMN measures (amplitude, theta ERO), with frontal left decreases (at F3) continuing to be related to larger MADRS decreases within Phase 2, in combination with less baseline left frontal PLF.

Several of these ketamine-induced changes were found to be predictive of decreases in early (left frontal MMN decreases in amplitude and theta ERO, baseline left PLF) and sustained 
(baseline left PLF, right inferior temporal activity) depressive symptoms, and were able to explain $0.45-.66$ of the variance. Together, these findings suggest that early and sustained ketamine responders may be characterized by less synchronized left phase-locking, more responsive NMDAR system indexed by acute decreases in MMN amplitude and theta ERO, as well as differentially characterize by activation in the right inferior temporal lobe.

In Sumner et al., (2020), ketamine induced higher forward connectivity modulation from the right primary auditory cortex to the right inferior temporal cortex (for deviant stimuli), which was correlated with greater decreases in depressive symptoms. As well, ketamine increased the strength of activation of the inferior temporal cortex. In the current study, less peak activity in $r$ rITL was related to change in depressive symptoms $2 \mathrm{hrs}$ postinfusion, while more peak activity in this region was related to change in depressive symptoms at the end of Phase 3 . This can be explained when considering that most patients were non-responders at $2 \mathrm{hrs}$ and most were responders at the end of Phase 3. As such, higher peak right interior temporal lobe activity was related to a greater antidepressant response.

\subsection{Limitations}

Several limitations should be considered. There were no MMN recordings at one day postinfusion, which is when the greatest response to ketamine are observed, or during the sustained treatment phases to assess longer-term changes in MMN measures following repeated and maintenance ketamine infusions. There was no separate baseline session outside the testing (preinfusion) days. Only one deviant was employed, therefore it cannot be determined whether differing patterns of ketamine-induced changes would have been observed with multiple deviant types. Additionally, the inherent spatial resolution limitations may have influenced the sourcelocalized MMN generator values. 
The participants in this study were limited to individuals enrolled in a larger clinical ketamine trial who were willing and able to participate in this electrophysiological component. Therefore, our sample was not large enough for a binomial analysis of responders vs. nonresponders and remitters vs. non-remitters at each time point. While midazolam is superior to saline as a placebo for ketamine, its lack of dissociative side effects may have influenced the blind within the first phase of the study. The patients in this study had a high level of illness severity and treatment-resistance, therefore the generalizability to other MDD populations may be limited.

Future studies would benefit from additional MMN recording time points which both acute and later response times. This would allow for more detailed assessments of the potential transition from an acute decreased state indexing NMDAR antagonism, to an increase representing enhanced synaptic plasticity. Analyses of ketamine-induced event-related synchronization/desynchronization, connectivity between MMN generators and/or between the default mode network and the salience network, as well as measures of cross-frequency coupling and their relation to the rapid and sustained antidepressant response would also be valuable.

\subsection{Conclusion}

To our knowledge, this is the first study to examine acute ketamine-induced MMN changes in relation to repeated ketamine infusions. Baseline and ketamine-induced decreases in multiple MMN-derived measures were related to and predictive of early and sustained decreases in depressive symptoms. Measures of MMN activity are thus feasible measures for investigating brain dynamics of NMDAR antagonism and synaptic plasticity resulting from sub-anesthetic doses of ketamine in TR MDD. 


\section{CHAPTER 4: GENERAL DISCUSSION}

\subsection{Summary}

This work sought to examine the electrophysiological effects of ketamine as compared to an active placebo (midazolam) in individuals with treatment-resistant MDD, as well as the relationship between these changes and decreases in depressive symptoms and suicidal ideation. Another goal was to expand on the existing literature on the use of baseline and early treatmentinduced changes in EEG measures in the prediction of treatment response and the characterization of treatment responders and non-responders. While most studies have examined early change within one to two weeks post-treatment initiation, ours examined these changes within an acute time window, given the rapid antidepressant effects associated with subanesthetic ketamine treatments.

Previous studies have explored ketamine's clinical effects, however, there is a paucity of work regarding acute and sustained alterations in brain electrical activity. The few electrophysiological studies that have been carried out with ketamine in MDD patients have recorded brain activity multiple hours postinfusion (Cornwell et al., 2012; Gilbert et al., 2018; Nugent et al., 2019a, 2019b). While this is closer to the one day posinfusion maximal clinical response time, the immediate effects on neurotransmission are critical for our understanding of treatment response characteristics. A novel component of the present work was the inclusion of two postinfusion recordings, immediately at the end of the infusion to capture acute physiological effects and a later recording, closer to the initiation of the rapid antidepressant effect, which has been found to begin as early as 2 hours postinfusion. These serve to fill the gaps in the literature of a lack of acute EEG recording in MDD samples. 
Few studies have examined repeated infusions for sustaining the antidepressant response. As such, there are no studies on the use of EEG biomarkers for sustained treatment response. This is critical for the potential to identify treatment responders before the administration of multiple infusions over the course of a few months.

\subsection{Ketamine's Effects on EEG-derived Measures}

Regional and frequency-specific changes were observed in both the ketamine and midazolam conditions. Both substances decreased alpha and theta immediately postinfusion. However, only midazolam increased delta immediately postinfusion, reflecting a sedative effect, and only ketamine increased gamma immediately post- and 2 hours postinfusion, reflecting increase cortical excitability. These findings were generally consistent with previous work. A strength of this work was the inclusion of all frequency bands (delta - gamma, sub-band divisions of theta and alpha), in addition to specific depression-related measures of alpha asymmetry, PF and MRF theta cordance, as well as source-localized theta current density at three ACC regional divisions (dorsal, rostral, subgenual). This allowed for the direct comparison of predictive measures within a TR MDD sample receiving multiple ketamine infusions.

\subsection{Ketamine's Effects on MMN Indices}

Ketamine reduced frontal MMN amplitudes; this is in line with previous human studies (Rosburg \& Kreitschmann-Andermahr, 2016). Decreases in theta ERO ( $\Delta$ postinfusion and $\Delta 2 \mathrm{hrs}$ ) and the frontal MMN generator ( $\Delta$ postinfusion only) were also found in the ketamine condition, potentially underlying the changes observed at the scalp-level. Contrary to our hypothesis, we did not observe increases in MMN measures at our later recording time ( $2 \mathrm{hrs}$ postinfusion), which may be due to our recording time point and/or our participant sample. At two hours postinfusion, the MMN may still be indexing a sustained reduction in amplitude due to 
NMDAR antagonism. The participants in our study also had severe TR MDD who mostly did not respond to the single infusion of Phase 1 (16.7\% responders in our subsample), and therefore may have a different neural response pattern as compared to less severely depressed individuals. With larger samples, comparisons could be made regarding depression severity level and subsequent increases vs. sustained decreases following sub-anesthetic ketamine infusions.

\subsection{Use of EEG/MMN Indices for Treatment Prediction}

A focus of extensive research has been the use of EEG and ERPs for antidepressant treatment response prediction. While our sample was too small to perform dichotomous analyses of treatment responders vs. non-responders and remitters vs. non-remitters, the examination of our EEG and MMN indices at baseline and their acute alterations with ketamine provide a preliminary exploration into possible candidate predictors for future trials.

Within the resting EEG study, alphal and alpha total power were found to be predictive of decrease in SI symptoms. However, when combined with baseline and early change in SI symptoms, these findings lost their significance. This supports the general findings of difficulty in predicting SI in TR MDD (Franklin et al., 2017). In the prediction of decrease in overall depressive symptoms, many of the strongest measures found in the current work (PF theta cordance, baseline rACC theta activity) have also been found previously with traditional pharmacotherapies (Iosifescu, 2011) and rTMS (Brunovsky et al., 2019; Hunter et al., 2018). In addition, potential ketamine-specific markers such as change in frontal gamma power, were also identified and should be examined in future trials.

As an exploratory examination of response at one day and at the end of Phase 2, baseline EEG measures were compared within responders and non-responders at these two critical time points indexing early response (when response to a single infusion is maximal) and sustained 
response (following 2 weeks of 3 infusions per week), respectively. Theta2 and total power at FMT as well as sgACC and rACC differed in early treatment responders, while sustained treatment responders differed in theta1 rACC activity. These findings support the use of theta sub-bands in electrophysiological prediction measures. However, additional work is needed in larger samples to confirm these differences.

Several baseline and ketamine-induced changes in MMN indices were found to be predictive of decreases in early (left frontal MMN decreases in amplitude and theta ERO, baseline left PLF) and sustained (baseline left PLF, right inferior temporal activity) depressive symptoms. Early and sustained ketamine responders may be characterized by less synchronized left phase-locking, more responsive NMDAR system indexed by acute decreases in MMN amplitude and theta ERO, as well as differentially characterize by activation in the right inferior temporal lobe. In addition, a better early response to a single sub-anesthetic ketamine administration was related to greater decreases in MMN measures (amplitude, theta ERO), with decreases in left frontal MMN amplitude continuing to be related to larger MADRS decreases within Phase 2, in combination with less baseline left frontal PLF. Higher baseline rITL peak MMN generator activity was predictive of sustained treatment response within repeated infusion responders (Phase 3).

These electrophysiological measures were, in many cases, more robust predictors of decreases in depressive symptoms than baseline and early change in MADRS scores (Figure 4.1). In the case of predicting response at one day postinfusion, the combination of electrophysiological measures (EEG: baseline theta2 sgACC and MMN: baseline left PLF) and MADRS measures ( $\triangle 2 \mathrm{hrs}$ MADRS) were more predictive together. This is in line with recent findings that a combination of different prediction measures is optimal for response prediction 
(Bares et al., 2015, 2017). Both EEG and MADRS scores are relatively easy to acquire (as compared to blood-based [invasive] or functional MRI [costly] biomarkers). Previous findings have shown that EEG-derived predictors alone (Wade \& Iosifescu, 2016) or depressive symptom predictors alone (Wagner et al., 2017) tend to yield modest predictive metrics.

\begin{tabular}{|c|c|c|c|}
\hline $\begin{array}{l}\text { Prediction } \\
\text { time point }\end{array}$ & EEG + MADRS & EEG + MADRS SI & MMN + MADRS \\
\hline$\Delta 2 \mathrm{hrs}$ & $\Delta$ post PF theta cordance & Baseline MADRS SI & $\begin{array}{c}\Delta \text { postinfusion MMN (F3) } \mu \mathrm{V}+ \\
\Delta \text { postinfusion ERO }\end{array}$ \\
\hline$\Delta 1 \mathrm{~d}$ & $\begin{array}{c}\triangle 2 \mathrm{hrs} \text { MADRS }+ \\
\text { Baseline sgACC theta2 }\end{array}$ & $\begin{array}{c}\triangle 2 \mathrm{hrs} \text { MADRS SI + } \\
\text { rACC theta2 baseline }\end{array}$ & $\begin{array}{l}\Delta 2 \mathrm{hrs} \text { MADRS + } \\
\text { Baseline left PLF } \\
\end{array}$ \\
\hline$\Delta \mathrm{P2}$ & Baseline rACC theta1 & $\Delta 2 \mathrm{hrs} \mathrm{MADRS} \mathrm{SI}$ & Baseline left PLF \\
\hline$\Delta \mathrm{Ph} 3$ & $\Delta 2 \mathrm{hrs}$ frontal gamma & Baseline MADRS SI & Baseline rITL CSD \\
\hline \multicolumn{4}{|c|}{ EEG/MMN only } \\
\hline \multicolumn{4}{|c|}{ MADRS only } \\
\hline \multicolumn{4}{|c|}{ MADRS+EEG/MMN } \\
\hline
\end{tabular}

Figure 4.1: Summary of most predictive variables at the four time points ( $2 \mathrm{hrs}, 1 \mathrm{~d}, \mathrm{Ph} 2, \mathrm{Ph} 3)$ for depressive and suicidal symptoms. $\Delta 2 \mathrm{hrs}=$ change from pre- to 2 hours postinfusion; $\Delta 1 \mathrm{~d}=$ change from pre- to 1 day postinfusion; $\Delta \mathrm{Ph} 2=$ change from baseline to end of phase $2 ; \Delta \mathrm{Ph} 3=$ change from baseline to end of phase 3; $\mathrm{CSD}=$ current source density; $\mathrm{ERO}=$ event-related oscillations; ITL = inferior temporal lobe; MADRS = Montgomery-Åsberg Depression Rating Scale; MADRS SI = Montgomery-Åsberg Depression Rating Scale item 10; MMN = mismatch negativity; $\mathrm{PF}=$ prefrontal; $\mathrm{PLF}=$ phase-locking factor; $\mathrm{r} / \mathrm{sgACC}=$ rostral/subgenual anterior cingulate cortex.

On a practical level, baseline EEG/MMN measures would be more useful for the prediction of early response ( $2 \mathrm{hrs}$ and $1 \mathrm{~d}$ post), as baseline measures can be performed without having to administer a ketamine infusion in order to calculate treatment-emergent change score. Additionally, once a single infusion has been administered in order to achieve a predictive change score, the patient and clinician will know within 24 hours whether there was a positive response or not. Both baseline and ketamine-induced changes could be useful in the prediction of sustained response (end of Phase 2/3), depending on the cost-benefit of administering a single 
infusion in order to acquire the change measure. In the current study, baseline theta $2 \mathrm{sgACC}$ and left MMN PLF would be most suitable for the prediction of early response, while theta1 rACC, left MMN PLF, right ITL activity, and change in frontal gamma power 2 hours postinfusion may be suitable for the prediction of sustained response (Figure 4.2). With larger studies and more consistent methodologies, predictive measures such as sensitivity, specificity, positive and negative predictive values, and overall accuracy may be calculated and an optimal cut-off point may be determined (Trevethan, 2017) for the potential routine differentiation of early and sustained treatment responders to sub-anesthetic ketamine.

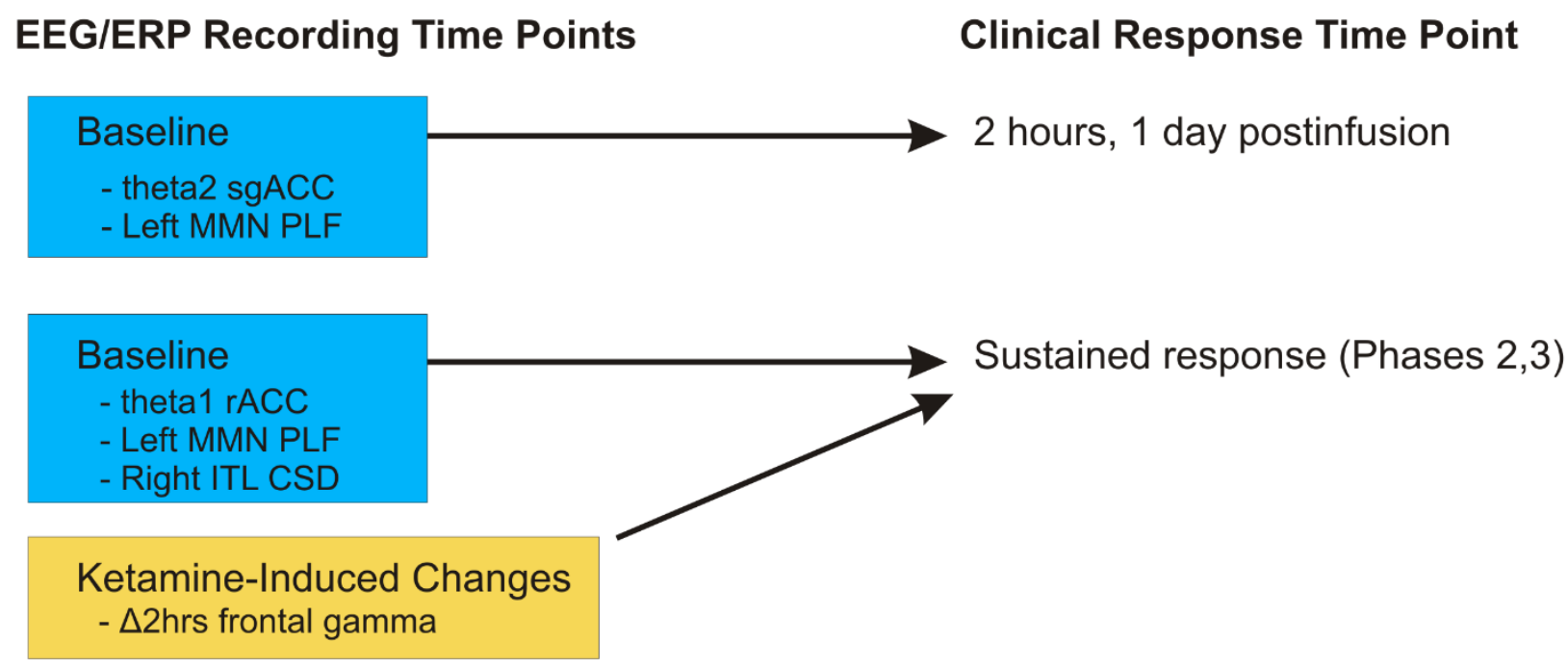

Figure 4.2: Optimal use of EEG/MMN measures in the prediction of antidepressant treatment response. $\mathrm{r} / \mathrm{sgACC}=$ rostral/subgenual anterior cingulate cortex; $\mathrm{MMN}=$ mismatch negativity; $\mathrm{PLF}=$ phase-locking factor; ITL $=$ inferior temporal lobe; $\mathrm{CSD}=$ current source density; $\Delta 2 \mathrm{hrs}=$ change from pre- to 2 hours postinfusion.

\subsection{Conclusion and Future Directions}

An increasing number of studies are being carried out globally to optimize the use of ketamine for TR MDD as well as many other conditions (bipolar disorder, social anxiety, post- 
traumatic stress disorder, etc.). Concurrently, research into the use of brain imaging for antidepressant treatment prediction has been steadily expanding and developing methodologically. These two fields are essential for understanding ketamine's clinical as well as neurophysiological actions. Many outstanding questions remain regarding ketamine's use as an antidepressant agent. Optimal enantiomer $(S, R$, racemic mixture) section, the role of active metabolites (e.g. HNK), method of delivery (i.v., intranasal, sublingual), patient demographics (sex, age, weight, race/ethnicity), and methods to sustain the antidepressant response (number of infusions, dose), are only a few potential areas of research. Future neuroimaging studies examining the antidepressant response to ketamine would benefit from multiple recording time points: immediately postinfusion to capture acute physiological effects, 2 hours postinfusion, when clinical effects begin, and one day postinfusion, when clinical effects are maximal. If examining sustained responses to ketamine, post-treatment recording times would be useful in quantifying EEG/ERP measure changes in responders and non-responders, and potentially assess remission and non-remission. Employing a three treatment group design involving ketamine, midazolam (or other active placebo substances), and saline may elucidate additional druginduced changes compared to ketamine vs. midazolam alone (Forsyth et al., 2018). In additional to measures of activity $\left(\mu \mathrm{V}, \mu \mathrm{V}^{2}, \mathrm{~A} / \mathrm{m}^{2}\right)$, assessing connectivity within and between key brain regions, in particular the regions of the triple network (CEN, SN, DMN), could contribute significantly to our understanding of how patterns of scalp- and surface-level oscillations are interlinked throughout the brain. Finally, the combination of multiple imaging modalities (e.g. EEG + fMRI) would allow for optimal temporal and spatial resolution.

In conclusion, this study provided novel electrophysiological information regarding acute effects of sub-anesthetic ketamine administered clinically in a sample of TR MDD, and their 
relation to depressive and SI symptoms. Given the increase in research of the clinical use of ketamine and the use of pharmaco-EEG for treatment response prediction, the findings of this work are extremely relevant and may aid future researchers in these fields. 


\section{REFERENCES}

aan het Rot, M., Collins, K. A., Murrough, J. W., Perez, A. M., Reich, D. L., Charney, D. S., \& Mathew, S. J. (2010). Safety and Efficacy of Repeated-Dose Intravenous Ketamine for Treatment-Resistant Depression. Biological Psychiatry, 67(2), 139-145. https://doi.org/10.1016/j.biopsych.2009.08.038

Abdallah, C. G., Sanacora, G., Duman, R. S., \& Krystal, J. H. (2015). Ketamine and rapid-acting antidepressants: A window into a new neurobiology for mood disorder therapeutics. Annual Review of Medicine, 66, 509-523. https://doi.org/10.1146/annurev-med-053013-062946

Aftanas, L. I., Varlamov, A. A., Pavlov, S. V., Makhnev, V. P., \& Reva, N. V. (2001). Affective picture processing: Event-related synchronization within individually defined human theta band is modulated by valence dimension. Neuroscience Letters, 303(2), 115-118. https://doi.org/10.1016/S0304-3940(01)01703-7

Alhaj, H., Wisniewski, G., \& McAllister-Williams, R. H. (2011). The use of the EEG in measuring therapeutic drug action: Focus on depression and antidepressants. Journal of Psychopharmacology, 25(9), 1175-1191. https://doi.org/10.1177/0269881110388323

American Psychiatric Association. (2000). 4th ed, text revision (DSM IV-TR). In Diagnostic and Statistical Manual of Mental Disorders (4th ed, te). American Psychiatric Publishing.

American Psychiatric Association. (2013). Diagnostic and Statistical Manual of Mental Disorders, 5th Edition. https://doi.org/10.1176/appi.books.9780890425596.dsm04

Angst, J., Angst, F., \& Stassen, H. H. (1999). Suicide risk in patients with major depressive disorder. The Journal of Clinical Psychiatry, 60 Suppl 2, 56-57,113-116.

Arnal, L. H. (2012). Predicting “when” using the motor system's beta-band oscillations. Frontiers in Human Neuroscience, 6. https://doi.org/10.3389/fnhum.2012.00225

Arns, M., Bruder, G., Hegerl, U., Spooner, C., Palmer, D. M., Etkin, A., Fallahpour, K., Gatt, J. M., Hirshberg, L., \& Gordon, E. (2016). EEG alpha asymmetry as a gender-specific predictor of outcome to acute treatment with different antidepressant medications in the randomized iSPOT-D study. Clinical Neurophysiology, 127(1), 509-519.

https://doi.org/10.1016/j.clinph.2015.05.032

Ballard, E. D., Luckenbaugh, D. A., Richards, E. M., Walls, T. L., Brutsché, N. E., Ameli, R., Niciu, M. J., Vande Voort, J. L., \& Zarate, C. A. (2015). Assessing measures of suicidal ideation in clinical trials with a rapid-acting antidepressant. Journal of Psychiatric Research, 68, 68-73. https://doi.org/10.1016/j.jpsychires.2015.06.003

Bares, M., Brunovsky, M., Kopecek, M., Novak, T., Stopkova, P., Kozeny, J., Sos, P., Krajca, V., \& Höschl, C. (2008). Early reduction in prefrontal theta QEEG cordance value predicts response to venlafaxine treatment in patients with resistant depressive disorder. European Psychiatry. https://doi.org/10.1016/j.eurpsy.2008.03.001

Bares, M., Brunovsky, M., Kopecek, M., Stopkova, P., Novak, T., Kozeny, J., \& Höschl, C. (2007). Changes in QEEG prefrontal cordance as a predictor of response to antidepressants in patients with treatment resistant depressive disorder: A pilot study. Journal of Psychiatric Research. https://doi.org/10.1016/j.jpsychires.2006.06.005 
Bares, M., Novak, T., Brunovsky, M., Kopecek, M., \& Höschl, C. (2017). The Comparison of Effectiveness of Various Potential Predictors of Response to Treatment with SSRIs in Patients with Depressive Disorder. Journal of Nervous and Mental Disease, 205(8), 618626. https://doi.org/10.1097/NMD.0000000000000574

Bares, M., Novak, T., Kopecek, M., Brunovsky, M., Stopkova, P., \& Höschl, C. (2015). The effectiveness of prefrontal theta cordance and early reduction of depressive symptoms in the prediction of antidepressant treatment outcome in patients with resistant depression: analysis of naturalistic data. European Archives of Psychiatry and Clinical Neuroscience, 265(1), 73-82. https://doi.org/10.1007/s00406-014-0506-8

Bares, M., Novak, T., Vlcek, P., Hejzlar, M., \& Brunovsky, M. (2019). Early change of prefrontal theta cordance and occipital alpha asymmetry in the prediction of responses to antidepressants. International Journal of Psychophysiology, 143, 1-8. https://doi.org/10.1016/j.ijpsycho.2019.06.006

Baskaran, A., Farzan, F., Milev, R., Brenner, C. A., Alturi, S., Pat McAndrews, M., Blier, P., Evans, K., Foster, J. A., Frey, B. N., Giacobbe, P., Lam, R. W., Leri, F., MacQueen, G. M., Müller, D. J., Parikh, S. V., Rotzinger, S., Soares, C. N., Strother, S. C., ... Kennedy, S. H. (2018). The comparative effectiveness of electroencephalographic indices in predicting response to escitalopram therapy in depression: A pilot study. Journal of Affective Disorders, 227, 542-549. https://doi.org/10.1016/j.jad.2017.10.028

BBC. (2011). Madagascar.

Beck, K., Hindley, G., Borgan, F., Ginestet, C., McCutcheon, R., Brugger, S., Driesen, N., Ranganathan, M., D’Souza, D. C., Taylor, M., Krystal, J. H., \& Howes, O. D. (2020). Association of Ketamine With Psychiatric Symptoms and Implications for Its Therapeutic Use and for Understanding Schizophrenia: A Systematic Review and Meta-analysis. JAMA Network Open, 3(5), e204693. https://doi.org/10.1001/jamanetworkopen.2020.4693

Benschop, L., Baeken, C., Vanderhasselt, M. A., van de Steen, F., van Heeringen, K., \& Arns, M. (2019). Electroencephalogram resting state frequency power characteristics of suicidal behavior in female patients with major depressive disorder. Journal of Clinical Psychiatry, 80(6). https://doi.org/10.4088/JCP.18m12661

Berchou, R., Chayasirisobhon, S., Green, V., \& Mason, K. (1986). The pharmacodynamic properties of lorazepam and methylphenidate drugs on event-related potentials and power spectral analysis in normal subjects. Clinical EEG Electroencephalography, 17(4), 176180. https://europepmc.org/article/med/3791644

Berman, R. M., Cappiello, A., Anand, A., Oren, D. A., Heninger, G. R., Charney, D. S., \& Krystal, J. H. (2000). Antidepressant effects of ketamine in depressed patients. Biological Psychiatry, 47(4), 351-354. https://doi.org/10.1016/S0006-3223(99)00230-9

Bi, K., Chattun, M. R., Liu, X., Wang, Q., Tian, S., Zhang, S., Lu, Q., \& Yao, Z. (2018). Abnormal early dynamic individual patterns of functional networks in low gamma band for depression recognition. Journal of Affective Disorders, 238, 366-374. https://doi.org/10.1016/j.jad.2018.05.078

Bissonnette, J. N., Francis, A. M., Hull, K. M., Leckey, J., Pimer, L., Berrigan, L. I., \& Fisher, D. 
J. (2020). MMN-Indexed Auditory Change Detection in Major Depressive Disorder. Clinical EEG and Neuroscience. https://doi.org/10.1177/1550059420914200

Blier, P., Zigman, D., \& Blier, J. (2012). On the safety and benefits of repeated intravenous injections of ketamine for depression. In Biological Psychiatry (Vol. 72, Issue 4). Biol Psychiatry. https://doi.org/10.1016/j.biopsych.2012.02.039

Bobo, W. V., Voort, J. L. V., Croarkin, P. E., Leung, J. G., Tye, S. J., \& Frye, M. A. (2016). Ketamine for treatment-resistant unipolar and bipolar major depression: critical review and implications for clinical practice. Depression and Anxiety, 33(8), 698-710. https://doi.org/10.1002/da.22505

Boku, S., Nakagawa, S., Toda, H., \& Hishimoto, A. (2018). Neural basis of major depressive disorder: Beyond monoamine hypothesis. Computer Graphics Forum, 37(2), 3-12. https://doi.org/10.1111/pcn.12604

Breimer, L. T. M., Hennis, P. J., Burm, A. G. L., Danhof, M., Bovill, J. G., Spierdijk, J., \& Vletter, A. A. (1990). Quantification of the EEG Effect of Midazolam by Aperiodic Analysis in Volunteers: Pharmacokinetic/Pharmacodynamic Modelling. Clinical Pharmacokinetics, 18(3), 245-253. https://doi.org/10.2165/00003088-199018030-00006

Broadway, J. M., Holtzheimer, P. E., Hilimire, M. R., Parks, N. A., Devylder, J. E., Mayberg, H. S., \& Corballis, P. M. (2012). Frontal theta cordance predicts 6-month antidepressant response to subcallosal cingulate deep brain stimulation for treatment-resistant depression: A pilot study. Neuropsychopharmacology. https://doi.org/10.1038/npp.2012.23

Bruder, G. E., Kayser, J., \& Tenke, C. E. (2012). Event-related brain potentials in depression: Clinical, cognitive, and neurophysiological implications. In The Oxford handbook of eventrelated potential components. (pp. 563-592). Oxford University Press.

Bruder, G. E., Sedoruk, J. P., Stewart, J. W., McGrath, P. J., Quitkin, F. M., \& Tenke, C. E. (2008). Electroencephalographic Alpha Measures Predict Therapeutic Response to a Selective Serotonin Reuptake Inhibitor Antidepressant: Pre- and Post-Treatment Findings. Biological Psychiatry, 63(12), 1171-1177. https://doi.org/10.1016/j.biopsych.2007.10.009

Bruder, G. E., Stewart, J. W., Tenke, C. E., McGrath, P. J., Leite, P., Bhattacharya, N., \& Quitkin, F. M. (2001). Electroencephalographic and perceptual asymmetry differences between responders and nonresponders to an SSRI antidepressant. Biological Psychiatry, 49(5), 416-425. https://doi.org/10.1016/S0006-3223(00)01016-7

Brunovsky, M., Bares, M., Novak, T., Kopecek, M., Horacek, J., Stopkova, P., \& Höschl, C. (2019). Comparable efficacy of prefrontal theta cordance in the prediction of response to antidepressants and rTMS. L'Encéphale, 45, S70-S71. https://doi.org/10.1016/j.encep.2019.04.020

Burcusa, S. L., \& Iacono, W. G. (2007). Risk for recurrence in depression. Clinical Psychology Review, 27(8), 959-985. https://doi.org/10.1016/j.cpr.2007.02.005

Buzśaki, G., \& Wang, X. J. (2012). Mechanisms of gamma oscillations. In Annual Review of Neuroscience (Vol. 35, pp. 203-225). NIH Public Access. https://doi.org/10.1146/annurevneuro-062111-150444 
Caddy, C., Amit, B. H., Mccloud, T. L., Rendell, J. M., Furukawa, T. A., Mcshane, R., Hawton, K., \& Cipriani, A. (2015). Ketamine and other glutamate receptor modulators for depression in adults. Cochrane Database of Systematic Reviews, 2015(9). https://doi.org/10.1002/14651858.CD011612.pub2

Cao, Z., Lin, C. T., Ding, W., Chen, M. H., Li, C. T., \& Su, T. P. (2019). Identifying Ketamine Responses in Treatment-Resistant Depression Using a Wearable Forehead EEG. IEEE Transactions on Biomedical Engineering, 66(6), 1668-1679. https://doi.org/10.1109/TBME.2018.2877651

Chennu, S., \& Bekinschtein, T. A. (2012). Arousal modulates auditory attention and awareness: Insights from sleep, sedation, and disorders of consciousness. In Frontiers in Psychology (Vol. 3, Issue MAR, p. 65). Frontiers. https://doi.org/10.3389/fpsyg.2012.00065

Cornwell, B. R., Salvadore, G., Furey, M., Marquardt, C. A., Brutsche, N. E., Grillon, C., \& Zarate, C. A. (2012). Synaptic potentiation is critical for rapid antidepressant response to ketamine in treatment-resistant major depression. Biological Psychiatry, 72(7), 555-561. https://doi.org/10.1016/j.biopsych.2012.03.029

Coyle, C. M., \& Laws, K. R. (2015). The use of ketamine as an antidepressant: A systematic review and meta-analysis. In Human Psychopharmacology (Vol. 30, Issue 3, pp. 152-163). John Wiley and Sons Ltd. https://doi.org/10.1002/hup.2475

Cuijpers, P., \& Schoevers, R. A. (2004). Increased mortality in depressive disorders: A review. Current Psychiatry Reports, 6(6), 430-437. https://doi.org/10.1007/s11920-004-0007-y

Cusin, C., Ionescu, D. F., Pavone, K. J., Akeju, O., Cassano, P., Taylor, N., Eikermann, M., Durham, K., Swee, M. B., Chang, T., Dording, C., Soskin, D., Kelley, J., Mischoulon, D., Brown, E. N., \& Fava, M. (2017). Ketamine augmentation for outpatients with treatmentresistant depression: Preliminary evidence for two-step intravenous dose escalation. Australian and New Zealand Journal of Psychiatry, 51(1), 55-64. https://doi.org/10.1177/0004867416631828

de la Salle, S., Choueiry, J., Shah, D., Bowers, H., McIntosh, J., Ilivitsky, V., \& Knott, V. (2016). Effects of ketamine on resting-state EEG activity and their relationship to perceptual/dissociative symptoms in healthy humans. Frontiers in Pharmacology, 7(SEP). https://doi.org/10.3389/fphar.2016.00348

de la Salle, S., Jaworska, N., Blier, P., Smith, D., \& Knott, V. (2020). Using prefrontal and midline right frontal EEG-derived theta cordance and depressive symptoms to predict the differential response or remission to antidepressant treatment in major depressive disorder. Psychiatry Research - Neuroimaging, 302. https://doi.org/10.1016/j.pscychresns.2020.111109

de la Salle, S., Shah, D., Choueiry, J., Bowers, H., McIntosh, J., Ilivitsky, V., \& Knott, V. (2019). NMDA receptor antagonist effects on speech-related mismatch negativity and its underlying oscillatory and source activity in healthy humans. Frontiers in Pharmacology, 10(MAY). https://doi.org/10.3389/fphar.2019.00455

De La Salle, S., Shah, D., Choueiry, J., Bowers, H., McIntosh, J., Ilivitsky, V., \& Knott, V. (2019). NMDA receptor antagonist effects on speech-related mismatch negativity and its 
underlying oscillatory and source activity in healthy humans. Frontiers in Pharmacology, 10(MAY). https://doi.org/10.3389/fphar.2019.00455

Deakin, J. F. W., Lees, J., McKie, S., Hallak, J. E. C., Williams, S. R., \& Dursun, S. M. (2008). Glutamate and the neural basis of the subjective effects of ketamine: A pharmaco-magnetic resonance imaging study. Archives of General Psychiatry, 65(2), 154-164. https://doi.org/10.1001/archgenpsychiatry.2007.37

Donner, T. H., \& Siegel, M. (2011). A framework for local cortical oscillation patterns. Trends in Cognitive Sciences, 15(5), 191-199. https://doi.org/10.1016/j.tics.2011.03.007

Downey, D., Dutta, A., McKie, S., Dawson, G. R., Dourish, C. T., Craig, K., Smith, M. A., McCarthy, D. J., Harmer, C. J., Goodwin, G. M., Williams, S., \& Deakin, J. F. W. (2016). Comparing the actions of lanicemine and ketamine in depression: Key role of the anterior cingulate. European Neuropsychopharmacology, 26(6), 994-1003. https://doi.org/10.1016/j.euroneuro.2016.03.006

Duncan, C. C., Barry, R. J., Connolly, J. F., Fischer, C., Michie, P. T., Näätänen, R., Polich, J., Reinvang, I., \& Van Petten, C. (2009). Event-related potentials in clinical research: Guidelines for eliciting, recording, and quantifying mismatch negativity, P300, and N400. Clinical Neurophysiology, 120(11), 1883-1908. https://doi.org/10.1016/j.clinph.2009.07.045

Fee, C., Banasr, M., \& Sibille, E. (2017). Somatostatin-Positive Gamma-Aminobutyric Acid Interneuron Deficits in Depression: Cortical Microcircuit and Therapeutic Perspectives. Biological Psychiatry, 82(8), 549-559. https://doi.org/10.1016/j.biopsych.2017.05.024

Feifel, D. (2016). Breaking Sad: Unleashing the Breakthrough Potential of Ketamine's Rapid Antidepressant Effects. Drug Development Research, 77(8), 489-494. https://doi.org/10.1002/ddr.21347

Ferrari, F., \& Villa, R. F. (2017). The Neurobiology of Depression: an Integrated Overview from Biological Theories to Clinical Evidence. Molecular Neurobiology, 54(7), 4847-4865. https://doi.org/10.1007/s12035-016-0032-y

Fitzgerald, P. J., \& Watson, B. O. (2018). Gamma oscillations as a biomarker for major depression: an emerging topic. Translational Psychiatry, 8(1), 177.

https://doi.org/10.1038/s41398-018-0239-y

Fond, G., Loundou, A., Rabu, C., Macgregor, A., Lançon, C., Brittner, M., Micoulaud-Franchi, J. A., Richieri, R., Courtet, P., Abbar, M., Roger, M., Leboyer, M., \& Boyer, L. (2014).

Ketamine administration in depressive disorders: A systematic review and meta-analysis. In Psychopharmacology (Vol. 231, Issue 18, pp. 3663-3676). Springer Verlag. https://doi.org/10.1007/s00213-014-3664-5

Forsyth, A., McMillan, R., Campbell, D., Malpas, G., Maxwell, E., Sleigh, J., Dukart, J., Hipp, J. F., \& Muthukumaraswamy, S. D. (2018). Comparison of local spectral modulation, and temporal correlation, of simultaneously recorded EEG/fMRI signals during ketamine and midazolam sedation. Psychopharmacology, 235(12), 3479-3493. https://doi.org/10.1007/s00213-018-5064-8 
Franklin, J. C., Ribeiro, J. D., Fox, K. R., Bentley, K. H., Kleiman, E. M., Huang, X., Musacchio, K. M., Jaroszewski, A. C., Chang, B. P., \& Nock, M. K. (2017). Risk factors for suicidal thoughts and behaviors: A meta-analysis of 50 years of research. Psychological Bulletin, 143(2), 187-232. https://doi.org/10.1037/bul0000084

Fried, E. (2017). Moving forward: how depression heterogeneity hinders progress in treatment and research. In Expert Review of Neurotherapeutics (Vol. 17, Issue 5, pp. 423-425). Taylor and Francis Ltd. https://doi.org/10.1080/14737175.2017.1307737

Fried, E., \& Nesse, R. (2015). Depression is not a consistent syndrome: An investigation of unique symptom patterns in the STAR $* \mathrm{D}$ study. Journal of Affective Disorders, 172, 96102. https://doi.org/10.1016/j.jad.2014.10.010

Frye, M. A., Blier, P., \& Tye, S. J. (2015). Implications for large scale study design and clinical development. Journal of Clinical Psychopharmacology, 35(3), 334-336. https://doi.org/10.1097/JCP.0000000000000316

Fu, C. H. Y., Steiner, H., \& Costafreda, S. G. (2013). Predictive neural biomarkers of clinical response in depression: A meta-analysis of functional and structural neuroimaging studies of pharmacological and psychological therapies. Neurobiology of Disease, 52, 75-83. https://doi.org/10.1016/j.nbd.2012.05.008

Fu, D. J., Ionescu, D. F., Li, X., Lane, R., Lim, P., Sanacora, G., Hough, D., Manji, H., Drevets, W. C., \& Canuso, C. M. (2020). Esketamine nasal spray for rapid reduction of major depressive disorder symptoms in patients who have active suicidal ideation with intent: Double-blind, randomized study (ASPIRE I). Journal of Clinical Psychiatry, 81(3). https://doi.org/10.4088/JCP.19m13191

Fuentemilla, L., Marco-Pallarés, J., Münte, T. F., \& Grau, C. (2008). Theta EEG oscillatory activity and auditory change detection. Brain Research, 1220, 93-101. https://doi.org/10.1016/j.brainres.2007.07.079

Gasser, T., Bächer, P., \& Möcks, J. (1982). Transformations towards the normal distribution of broad band spectral parameters of the EEG. Electroencephalography and Clinical Neurophysiology, 53(1), 119-124. https://doi.org/10.1016/0013-4694(82)90112-2

Gil-Da-Costa, R., Stoner, G. R., Fung, R., \& Albright, T. D. (2013). Nonhuman primate model of schizophrenia using a noninvasive EEG method. Proceedings of the National Academy of Sciences of the United States of America, 110(38), 15425-15430. https://doi.org/10.1073/pnas.1312264110

Gilbert, J. R., Yarrington, J. S., Wills, K. E., Nugent, A. C., \& Zarate, C. A. (2018). Glutamatergic signaling drives ketamine-mediated response in depression: Evidence from dynamic causal modeling. International Journal of Neuropsychopharmacology, 21(8), 740747. https://doi.org/10.1093/ijnp/pyy041

Gilbert, J. R., \& Zarate, C. A. (2020). Electrophysiological biomarkers of antidepressant response to ketamine in treatment-resistant depression: Gamma power and long-term potentiation. Pharmacology Biochemistry and Behavior, 189. https://doi.org/10.1016/j.pbb.2020.172856 
Glenn, C. R., \& Nock, M. K. (2014). Improving the short-term prediction of suicidal behavior. American Journal of Preventive Medicine, 47(3 SUPPL. 2). https://doi.org/10.1016/j.amepre.2014.06.004

Gratton, G., Coles, M. G. H., \& Donchin, E. (1983). A new method for off-line removal of ocular artifact. Electroencephalography and Clinical Neurophysiology, 55(4), 468-484. https://doi.org/10.1016/0013-4694(83)90135-9

Grin-Yatsenko, V. A., Baas, I., Ponomarev, V. A., \& Kropotov, J. D. (2009). EEG power spectra at early stages of depressive disorders. Journal of Clinical Neurophysiology, 26(6), 401406. https://doi.org/10.1097/WNP.0b013e3181c298fe

Grunebaum, M. F., Galfalvy, H. C., Choo, T. H., Keilp, J. G., Moitra, V. K., Parris, M. S., Marver, J. E., Burke, A. K., Milak, M. S., Sublette, M. E., Oquendo, M. A., \& Mann, J. J. (2018). Ketamine for rapid reduction of suicidal thoughts in major depression: A midazolam-controlled randomized clinical trial. American Journal of Psychiatry, 175(4), 327-335. https://doi.org/10.1176/appi.ajp.2017.17060647

Gunduz-Bruce, H., Reinhart, R. M. G., Roach, B. J., Gueorguieva, R., Oliver, S., D’Souza, D. C., Ford, J. M., Krystal, J. H., \& Mathalon, D. H. (2012). Glutamatergic modulation of auditory information processing in the human brain. Biological Psychiatry, 71(11), 969977. https://doi.org/10.1016/j.biopsych.2011.09.031

Hajcak, G., Klawohn, J., \& Meyer, A. (2019). The Utility of Event-Related Potentials in Clinical Psychology. In Annual Review of Clinical Psychology (Vol. 15, pp. 71-95). Annual Reviews Inc. https://doi.org/10.1146/annurev-clinpsy-050718-095457

Hall, M. H., Taylor, G., Salisbury, D. F., \& Levy, D. L. (2011). Sensory gating event-related potentials and oscillations in schizophrenia patients and their unaffected relatives. Schizophrenia Bulletin, 37(6), 1187-1199. https://doi.org/10.1093/schbul/sbq027

Hammer-Helmich, L., Haro, J. M., Jönsson, B., Melac, A. T., Di Nicola, S., Chollet, J., Milea, D., Rive, B., \& Saragoussi, D. (2018). Functional impairment in patients with major depressive disorder: The 2-year PERFORM study. Neuropsychiatric Disease and Treatment, 14, 239-249. https://doi.org/10.2147/NDT.S146098

Harmon-Jones, E., \& Gable, P. A. (2018). On the role of asymmetric frontal cortical activity in approach and withdrawal motivation: An updated review of the evidence. Psychophysiology, 55(1). https://doi.org/10.1111/psyp.12879

Hashimoto, K., Sawa, A., \& Iyo, M. (2007). Increased Levels of Glutamate in Brains from Patients with Mood Disorders. Biological Psychiatry, 62(11), 1310-1316. https://doi.org/10.1016/j.biopsych.2007.03.017

He, W., Chai, H., Zheng, L., Yu, W., Chen, W., Li, J., Chen, W., \& Wang, W. (2010). Mismatch negativity in treatment-resistant depression and borderline personality disorder. Progress in Neuro-Psychopharmacology and Biological Psychiatry, 34(2), 366-371. https://doi.org/10.1016/j.pnpbp.2009.12.021

Heekeren, K., Daumann, J., Neukirch, A., Stock, C., Kawohl, W., Norra, C., Waberski, T. D., \& Gouzoulis-Mayfrank, E. (2008). Mismatch negativity generation in the human 5HT2A 
agonist and NMDA antagonist model of psychosis. Psychopharmacology, 199(1), 77-88. https://doi.org/10.1007/s00213-008-1129-4

Hegerl, U., Wilk, K., Olbrich, S., Schoenknecht, P., \& Sander, C. (2012). Hyperstable regulation of vigilance in patients with major depressive disorder. World Journal of Biological Psychiatry, 13(6), 436-446. https://doi.org/10.3109/15622975.2011.579164

Heller, W., \& Nitscke, J. B. (1997). regional Brain Activity in Emotion: A Framework for Understanding Cognition in Depresion. Cognition and Emotion, 11(5-6), 637-661. https://doi.org/10.1080/026999397379845a

Hering, W., Geisslinger, G., Kamp, H. D., Dinkel, M., Tschaikowsky, K., Rugheimer, E., \& Brune, K. (1994). Changes in the EEG power spectrum after midazolam anaesthesia combined with racemic or S - (+) ketamine. Acta Anaesthesiologica Scandinavica, 38(7), 719-723. https://doi.org/10.1111/j.1399-6576.1994.tb03984.x

Hillhouse, T. M., \& Porter, J. H. (2015). A brief history of the development of antidepressant drugs: From monoamines to glutamate. Experimental and Clinical Psychopharmacology, 23(1), 1-21. https://doi.org/10.1037/a0038550

Hirschfeld, R. M. (2000). History and evolution of the monoamine hypothesis of depression. The Journal of Clinical Psychiatry, 61 Suppl 6, 4-6.

Hofmann-Shen, C., Vogel, B. O., Kaffes, M., Rudolph, A., Brown, E. C., Tas, C., Brüne, M., \& Neuhaus, A. H. (2020). Mapping adaptation, deviance detection, and prediction error in auditory processing. NeuroImage, 207, 116432.

https://doi.org/10.1016/j.neuroimage.2019.116432

Holsboer, F. (2008). How can we realize the promise of personalized antidepressant medicines? In Nature Reviews Neuroscience (Vol. 9, Issue 8, pp. 638-646). Nat Rev Neurosci. https://doi.org/10.1038/nrn2453

Horacek, J., Brunovsky, M., Novak, T., Tislerova, B., Palenicek, T., Bubenikova-Valesova, V., Spaniel, F., Koprivova, J., Mohr, P., Balikova, M., \& Hoschl, C. (2010). Subanesthetic dose of ketamine decreases prefrontal theta cordance in healthy volunteers: Implications for antidepressant effect. Psychological Medicine, 40(9), 1443-1451. https://doi.org/10.1017/S0033291709991619

Hotz, M. A., Ritz, R., Linder, L., Scollo-Lavizzari, G., \& Haefeli, W. E. (2000). Auditory and electroencephalographic effects of midazolam and $\alpha$-hydroxy-midazolam in healthy subjects. British Journal of Clinical Pharmacology, 49(1), 72-79.

https://doi.org/10.1046/j.1365-2125.2000.00104.x

Hunt, M. J., \& Kasicki, S. (2013). A systematic review of the effects of NMDA receptor antagonists on oscillatory activity recorded in vivo. Journal of Psychopharmacology, 27(11), 972-986. https://doi.org/10.1177/0269881113495117

Hunter, A. M., Nghiem, T. X., Cook, I. A., Krantz, D. E., Minzenberg, M. J., \& Leuchter, A. F. (2018). Change in Quantitative EEG Theta Cordance as a Potential Predictor of Repetitive Transcranial Magnetic Stimulation Clinical Outcome in Major Depressive Disorder. Clinical EEG and Neuroscience, 49(5), 306-315. 
https://doi.org/10.1177/1550059417746212

IBM. (2016). IBM SPSS Statistics for Windows, Version 24.0. IBM Corp.

Iosifescu, D. V. (2011). Electroencephalography-derived biomarkers of antidepressant response. Harvard Review of Psychiatry, 19(3), 144-154. https://doi.org/10.3109/10673229.2011.586549

Iosifescu, D. V., Greenwald, S., Devlin, P., Mischoulon, D., Denninger, J. W., Alpert, J. E., \& Fava, M. (2009). Frontal EEG predictors of treatment outcome in major depressive disorder. European Neuropsychopharmacology, 19(11), 772-777. https://doi.org/10.1016/j.euroneuro.2009.06.001

Jaworska, N., Blier, P., Fusee, W., \& Knott, V . (2012). Alpha power, alpha asymmetry and anterior cingulate cortex activity in depressed males and females. Journal of Psychiatric Research, 46(11), 1483-1491. https://doi.org/10.1016/j.jpsychires.2012.08.003

Jaworska, N., De La Salle, S., Ibrahim, M.-H., Blier, P., \& Knott, V. (2019). Leveraging machine learning approaches for predicting antidepressant treatment response using electroencephalography (EEG) and clinical data. Frontiers in Psychiatry, 10(JAN). https://doi.org/10.3389/fpsyt.2018.00768

Jensen, O., Kaiser, J., \& Lachaux, J. P. (2007). Human gamma-frequency oscillations associated with attention and memory. Trends in Neurosciences, 30(7), 317-324. https://doi.org/10.1016/j.tins.2007.05.001

Jobert, M., Wilson, F. J., Ruigt, G. S. F., Brunovsky, M., Prichep, L. S., \& Drinkenburg, W. H. I. M. (2012). Guidelines for the recording and evaluation of pharmaco-eeg data in man: The international pharmaco-EEG society (IPEG): The IPEG pharmaco-EEG guideline committee. Neuropsychobiology, 66(4), 201-220. https://doi.org/10.1159/000343478

Just, M. A., Pan, L., Cherkassky, V. L., McMakin, D. L., Cha, C., Nock, M. K., \& Brent, D. (2017). Machine learning of neural representations of suicide and emotion concepts identifies suicidal youth. Nature Human Behaviour, 1(12), 911-919. https://doi.org/10.1038/s41562-017-0234-y

Kähkönen, S., Yamashita, H., Rytsälä, H., Suominen, K., Ahveninen, J., \& Isometsä, E. (2007). Dysfunction in early auditory processing in major depressive disorder revealed by combined MEG and EEG. Journal of Psychiatry \& Neuroscience : JPN, 32(5), 316-322.

Kantrowitz, J. T., Swerdlow, N. R., Dunn, W., \& Vinogradov, S. (2018). Auditory System Target Engagement During Plasticity-Based Interventions in Schizophrenia: A Focus on Modulation of N-Methyl-D-Aspartate-Type Glutamate Receptor Function. In Biological Psychiatry: Cognitive Neuroscience and Neuroimaging (Vol. 3, Issue 7, pp. 581-590). Elsevier Inc. https://doi.org/10.1016/j.bpsc.2018.02.002

Karakaş, S. (2020). A review of theta oscillation and its functional correlates. International Journal of Psychophysiology. https://doi.org/10.1016/j.ijpsycho.2020.04.008

Kaser, M., Soltesz, F., Lawrence, P., Miller, S., Dodds, C., Croft, R., Dudas, R. B., Zaman, R., Fernandez-Egea, E., Müller, U., Dean, A., Bullmore, E. T., \& Nathan, P. J. (2013). Oscillatory underpinnings of mismatch negativity and their relationship with cognitive 
function in patients with schizophrenia. PLOS ONE.

https://doi.org/10.1371/journal.pone.0083255

Kasper, S. (2014). Treatment-resistant depression: A challenge for future research. Acta Neuropsychiatrica, 26(3), 131-133. https://doi.org/10.1017/neu.2014.8

Kennedy, S. H., Eisfeld, B. S., Meyer, J. H., \& Bagby, R. M. (2001). Antidepressants in clinical practice: limitations of assessment methods and drug response. Human Psychopharmacology, 16(1), 105-114. https://doi.org/10.1002/hup.189

Kennedy, S. H., Konarski, J. Z., Segal, Z. V., Lau, M. A., Bieling, P. J., McIntyre, R. S., \& Mayberg, H. S. (2007). Differences in brain glucose metabolism between responders to CBT and venlafaxine in a 16-week randomized controlled trial. American Journal of Psychiatry, 164(5), 778-788. https://doi.org/10.1176/ajp.2007.164.5.778

Kilavik, B. E., Zaepffel, M., Brovelli, A., MacKay, W. A., \& Riehle, A. (2013). The ups and downs of beta oscillations in sensorimotor cortex. Experimental Neurology, 245, 15-26. https://doi.org/10.1016/j.expneurol.2012.09.014

Kim, S., Baek, J. H., Shim, S. hoon, Kwon, Y. J., Lee, H. Y., Yoo, J. H., \& Kim, J. S. (2020). Mismatch negativity indices and functional outcomes in unipolar and bipolar depression. Scientific Reports, 10(1). https://doi.org/10.1038/s41598-020-69776-4

Kishimoto, T., Chawla, J. M., Hagi, K., Zarate, C. A., Kane, J. M., Bauer, M., \& Correll, C. U. (2016). Single-dose infusion ketamine and non-ketamine N-methyl-d-aspartate receptor antagonists for unipolar and bipolar depression: A meta-analysis of efficacy, safety and time trajectories. Psychological Medicine, 46(7), 1459-1472. https://doi.org/10.1017/S0033291716000064

Klimesch, W. (1999). EEG alpha and theta oscillations reflect cognitive and memory performance: A review and analysis. Brain Research Reviews, 29(2-3), 169-195. https://doi.org/10.1016/S0165-0173(98)00056-3

Klimesch, W. (2012). Alpha-band oscillations, attention, and controlled access to stored information. Trends in Cognitive Sciences, 16(12), 606-617. https://doi.org/10.1016/j.tics.2012.10.007

Klimesch, W., Sauseng, P., \& Hanslmayr, S. (2007). EEG alpha oscillations: The inhibitiontiming hypothesis. Brain Research Reviews, 53(1), 63-88. https://doi.org/10.1016/j.brainresrev.2006.06.003

Klonsky, E. D., May, A. M., \& Saffer, B. Y. (2016). Suicide, Suicide Attempts, and Suicidal Ideation. Annual Review of Clinical Psychology, 12(1), 307-330. https://doi.org/10.1146/annurev-clinpsy-021815-093204

Knott, V. J., Telner, J. I., Lapierre, Y. D., Browne, M., \& Horn, E. R. (1996). Quantitative EEG in the prediction of antidepressant response to imipramine. Journal of Affective Disorders, 39(3), 175-184. https://doi.org/10.1016/0165-0327(96)00003-1

Knott, V., Mahoney, C., Kennedy, S., \& Evans, K. (2000). Pre-treatment EEG and it's relationship to depression severity and paroxetine treatment outcome. Pharmacopsychiatry, 33(6), 201-205. https://doi.org/10.1055/s-2000-8356 
Knott, V., Mahoney, C., Kennedy, S., \& Evans, K. (2001). EEG power, frequency, asymmetry and coherence in male depression. Psychiatry Research - Neuroimaging, 106(2), 123-140. https://doi.org/10.1016/S0925-4927(00)00080-9

Knott, V., McIntosh, J., Millar, A., Fisher, D., Villeneuve, C., Ilivitsky, V., \& Horn, E. (2006). Nicotine and smoker status moderate brain electric and mood activation induced by ketamine, an N-methyl-d-aspartate (NMDA) receptor antagonist. Pharmacology Biochemistry and Behavior, 85(1), 228-242. https://doi.org/10.1016/j.pbb.2006.08.005

Knyazev, G. G. (2012). EEG delta oscillations as a correlate of basic homeostatic and motivational processes. Neuroscience and Biobehavioral Reviews, 36(1), 677-695. https://doi.org/10.1016/j.neubiorev.2011.10.002

Kochs, E., Scharein, E., Möllenberg, O., Bromm, B., \& Schulte am Esch, J. (1996). Analgesic efficacy of low-dose ketamine: Somatosensory-evoked responses in relation to subjective pain ratings. Anesthesiology, 85(2), 304-314. https://doi.org/10.1097/00000542199608000-00012

König, H., König, H. H., \& Konnopka, A. (2019). The excess costs of depression: A systematic review and meta-analysis. In Epidemiology and Psychiatric Sciences (Vol. 29). Cambridge University Press. https://doi.org/10.1017/S2045796019000180

Kreitschmann-Andermahr, I., Rosburg, T., Demme, U., Gaser, E., Nowak, H., \& Sauer, H. (2001). Effect of ketamine on the neuromagnetic mismatch field in healthy humans. Cognitive Brain Research, 12(1), 109-116. https://doi.org/10.1016/S0926-6410(01)00043$\mathrm{X}$

Kuizenga, K., Wierda, J. M. K. H., \& Kalkman, C. J. (2001). Biphasic EEG changes in relation to loss of consciousness during induction with thiopental, propofol, etomidate, midazolam or sevoflurane. British Journal of Anaesthesia, 86(3), 354-360. https://doi.org/10.1093/bja/86.3.354

Kwon, J. S., Youn, T., \& Jung, H. Y. (1996). Right hemisphere abnormalities in major depression: Quantitative electroencephalographic findings before and after treatment. Journal of Affective Disorders, 40(3), 169-173. https://doi.org/10.1016/01650327(96)00057-2

Labermaier, C., Masana, M., \& Müller, M. B. (2013). Biomarkers predicting antidepressant treatment response: How can we advance the field? In Disease Markers (Vol. 35, Issue 1, pp. 23-31). Hindawi Limited. https://doi.org/10.1155/2013/984845

Lai, C. H. (2019). Promising neuroimaging biomarkers in depression. Psychiatry Investigation, 16(9), 662-670. https://doi.org/10.30773/pi.2019.07.25.2

Lange, J., Oostenveld, R., \& Fries, P. (2013). Reduced occipital alpha power indexes enhanced excitability rather than improved visual perception. Journal of Neuroscience, 33(7), 32123220. https://doi.org/10.1523/JNEUROSCI.3755-12.2013

Laufs, H., Kleinschmidt, A., Beyerle, A., Eger, E., Salek-Haddadi, A., Preibisch, C., \& Krakow, K. (2003). EEG-correlated fMRI of human alpha activity. NeuroImage, 19(4), 1463-1476. https://doi.org/10.1016/S1053-8119(03)00286-6 
Lee, M., Balla, A., Sershen, H., Sehatpour, P., Lakatos, P., \& Javitt, D. C. (2018). Rodent Mismatch Negativity/theta Neuro-Oscillatory Response as a Translational Neurophysiological Biomarker for N-Methyl-D-Aspartate Receptor-Based New Treatment Development in Schizophrenia. Neuropsychopharmacology, 43(3), 571-582. https://doi.org/10.1038/npp.2017.176

Lee, P. S., Chen, Y. S., Hsieh, J. C., Su, T. P., \& Chen, L. F. (2010). Distinct neuronal oscillatory responses between patients with bipolar and unipolar disorders: A magnetoencephalographic study. Journal of Affective Disorders, 123(1-3), 270-275. https://doi.org/10.1016/j.jad.2009.08.020

Lee, S. M., Jang, K. I., \& Chae, J. H. (2017). Electroencephalographic Correlates of Suicidal Ideation in the Theta Band. Clinical EEG and Neuroscience, 48(5), 316-321. https://doi.org/10.1177/1550059417692083

Lener, M. S., Niciu, M. J., Ballard, E. D., Park, M., Park, L. T., Nugent, A. C., \& Zarate, C. A. (2017). Glutamate and Gamma-Aminobutyric Acid Systems in the Pathophysiology of Major Depression and Antidepressant Response to Ketamine. Biological Psychiatry, 81(10), 886-897. https://doi.org/10.1016/j.biopsych.2016.05.005

Leuchter, A. F., Cook, I. A., DeBrota, D. J., Hunter, A. M., Potter, W. Z., McGrouther, C. C., Morgan, M. L., Abrams, M., \& Siegman, B. (2008). Changes in brain function during administration of venlafaxine or placebo to normal subjects. Clinical EEG and Neuroscience, 39(4), 175-181. https://doi.org/10.1177/155005940803900405

Leuchter, A. F., Uijtdehaage, S. H. J., Cook, I. A., O’Hara, R., \& Mandelkern, M. (1999). Relationship between brain electrical activity and cortical perfusion in normal subjects. Psychiatry Research - Neuroimaging, 90(2), 125-140. https://doi.org/10.1016/S09254927(99)00006-2

Light, G. A., Williams, L. E., Minow, F., Sprock, J., Rissling, A., Sharp, R., Swerdlow, N. R., \& Braff, D. L. (2010). Electroencephalography (EEG) and event-related potentials (ERPs) with human participants. In Current Protocols in Neuroscience: Vol. Chapter 6 (Issue SUPPL. 52). Curr Protoc Neurosci. https://doi.org/10.1002/0471142301.ns0625s52

Liu, T. Y., Chen, Y. S., Su, T. P., Hsieh, J. C., \& Chen, L. F. (2014). Abnormal early gamma responses to emotional faces differentiate unipolar from bipolar disorder patients. BioMed Research International, 2014. https://doi.org/10.1155/2014/906104

Liu, T. Y., Hsieh, J. C., Chen, Y. S., Tu, P. C., Su, T. P., \& Chen, L. F. (2012). Different patterns of abnormal gamma oscillatory activity in unipolar and bipolar disorder patients during an implicit emotion task. Neuropsychologia, 50(7), 1514-1520. https://doi.org/10.1016/j.neuropsychologia.2012.03.004

Luck, S. J. (2012). Event-related potentials. In APA handbook of research methods in psychology, Vol 1: Foundations, planning, measures, and psychometrics. (pp. 523-546). American Psychological Association. https://doi.org/10.1037/13619-028

Lye, M. S., Tey, Y. Y., Tor, Y. S., Shahabudin, A. F., Ibrahimid, N., Ling, K. H., Stanslas, J., Loh, S. P., Rosli, R., Lokman, K. A., Badamasi, I. M., Faris-Aldoghachi, A., \& Razak, N. A. A. (2020). Predictors of recurrence of major depressive disorder. PLoS ONE, 15(3). 
https://doi.org/10.1371/journal.pone.0230363

MacLean, S. E., Blundon, E. G., \& Ward, L. M. (2015). Brain regional networks active during the mismatch negativity vary with paradigm. Neuropsychologia, 75, 242-251. https://doi.org/10.1016/j.neuropsychologia.2015.06.019

Mathalon, D. H., Ahn, K. H., Perry, E. B., Cho, H. S., Roach, B. J., Blais, R. K., Bhakta, S., Ranganathan, M., Ford, J. M., \& D'Souza, D. C. (2014). Effects of nicotine on the neurophysiological and behavioral effects of ketamine in humans. Frontiers in Psychiatry, 5(JAN). https://doi.org/10.3389/fpsyt.2014.00003

Mathews, D. C., Henter, I. D., \& Zarate, C. A. (2012). Targeting the glutamatergic system to treat major depressive disorder: Rationale and progress to date. Drugs, 72(10), 1313-1333. https://doi.org/10.2165/11633130-000000000-00000

Mazziotta, J., Toga, A., Evans, A., Fox, P., Lancaster, J., Zilles, K., Woods, R., Paus, T., Simpson, G., Pike, B., Holmes, C., Collins, L., Thompson, P., MacDonald, D., Iacoboni, M., Schormann, T., Amunts, K., Palomero-Gallagher, N., Geyer, S., ... Mazoyer, B. (2001). A probabilistic atlas and reference system for the human brain: International Consortium for Brain Mapping (ICBM). Philosophical Transactions of the Royal Society B: Biological Sciences, 356(1412), 1293-1322. https://doi.org/10.1098/rstb.2001.0915

McMillan, R., Forsyth, A., Campbell, D., Malpas, G., Maxwell, E., Dukart, J., Hipp, J. F., \& Muthukumaraswamy, S. (2019). Temporal dynamics of the pharmacological MRI response to subanaesthetic ketamine in healthy volunteers: A simultaneous EEG/fMRI study. Journal of Psychopharmacology, 33(2), 219-229. https://doi.org/10.1177/0269881118822263

McMillan, R., \& Muthukumaraswamy, S. D. (2020). The neurophysiology of ketamine: An integrative review. Reviews in the Neurosciences, 31(5), 457-503. https://doi.org/10.1515/revneuro-2019-0090

McMillan, R., Sumner, R., Forsyth, A., Campbell, D., Malpas, G., Maxwell, E., Deng, C., Hay, J., Ponton, R., Sundram, F., \& Muthukumaraswamy, S. (2020). Simultaneous EEG/fMRI recorded during ketamine infusion in patients with major depressive disorder. Progress in Neuro-Psychopharmacology and Biological Psychiatry, 99. https://doi.org/10.1016/j.pnpbp.2019.109838

Mitchell, D. J., McNaughton, N., Flanagan, D., \& Kirk, I. J. (2008). Frontal-midline theta from the perspective of hippocampal "theta." In Progress in Neurobiology (Vol. 86, Issue 3, pp. 156-185). Pergamon. https://doi.org/10.1016/j.pneurobio.2008.09.005

Monroe, S. M., \& Harkness, K. L. (2011). Recurrence in Major Depression: A Conceptual Analysis. Psychological Review, 118(4), 655-674. https://doi.org/10.1037/a0025190

Montgomery, S. A., \& Asberg, M. (1979). A new depression scale designed to be sensitive to change. British Journal of Psychiatry, 134(4), 382-389.

https://doi.org/10.1192/bjp.134.4.382

Morgan, M. L., Witte, E. A., Cook, I. A., Leuchter, A. F., Abrams, M., \& Siegman, B. (2005). Influence of age, gender, health status, and depression on quantitative EEG.

Neuropsychobiology, 52(2), 71-76. https://doi.org/10.1159/000086608 
Mucci, A., Volpe, U., Merlotti, E., Bucci, P., \& Galderisi, S. (2006). Pharmaco-EEG in psychiatry. In Clinical EEG and Neuroscience (Vol. 37, Issue 2, pp. 81-98). EEG and Clinical Neuroscience Society (ECNS). https://doi.org/10.1177/155005940603700206

Mulert, C., Jäger, L., Schmitt, R., Bussfeld, P., Pogarell, O., Möller, H. J., Juckel, G., \& Hegerl, U. (2004). Integration of fMRI and simultaneous EEG: Towards a comprehensive understanding of localization and time-course of brain activity in target detection. NeuroImage, 22(1), 83-94. https://doi.org/10.1016/j.neuroimage.2003.10.051

Murrough, J. W., Abdallah, C. G., \& Mathew, S. J. (2017). Targeting glutamate signalling in depression: Progress and prospects. In Nature Reviews Drug Discovery (Vol. 16, Issue 7, pp. 472-486). Nature Publishing Group. https://doi.org/10.1038/nrd.2017.16

Murrough, J. W., Iosifescu, D. V., Chang, L. C., Al Jurdi, R. K., Green, C. E., Perez, A. M., Iqbal, S., Pillemer, S., Foulkes, A., Shah, A., Charney, D. S., \& Mathew, S. J. (2013a). Antidepressant efficacy of ketamine in treatment-resistant major depression: A two-site randomized controlled trial. American Journal of Psychiatry, 170(10), 1134-1142. https://doi.org/10.1176/appi.ajp.2013.13030392

Murrough, J. W., Perez, A. M., Pillemer, S., Stern, J., Parides, M. K., Aan Het Rot, M., Collins, K. A., Mathew, S. J., Charney, D. S., \& Iosifescu, D. V. (2013b). Rapid and longer-term antidepressant effects of repeated ketamine infusions in treatment-resistant major depression. Biological Psychiatry, 74(4), 250-256. https://doi.org/10.1016/j.biopsych.2012.06.022

Murrough, J. W., Soleimani, L., Dewilde, K. E., Collins, K. A., Lapidus, K. A., Iacoviello, B. M., Lener, M., Kautz, M., Kim, J., Stern, J. B., Price, R. B., Perez, A. M., Brallier, J. W., Rodriguez, G. J., Goodman, W. K., Iosifescu, D. V., \& Charney, D. S. (2015). Ketamine for rapid reduction of suicidal ideation: A randomized controlled trial. Psychological Medicine, 45(16), 3571-3580. https://doi.org/10.1017/S0033291715001506

Muthukumaraswamy, S. D., Shaw, A. D., Jackson, L. E., Hall, J., Moran, R., \& Saxena, N. (2015). Evidence that subanesthetic doses of ketamine cause sustained disruptions of NMDA and AMPA-mediated frontoparietal connectivity in humans. Journal of Neuroscience, 35(33), 11694-11706. https://doi.org/10.1523/JNEUROSCI.0903-15.2015

Näätänen, R. (1990). The role of attention in auditory information processing as revealed by event-related potentials and other brain measures of cognitive function. Behavioral and Brain Sciences, 13(2), 201-233. https://doi.org/10.1017/S0140525X00078407

Näätänen, R., Paavilainen, P., Rinne, T., \& Alho, K. (2007). The mismatch negativity (MMN) in basic research of central auditory processing: A review. Clinical Neurophysiology, 118(12), 2544-2590. https://doi.org/10.1016/j.clinph.2007.04.026

Näätänen, R., \& Picton, T. (1987). The N1 Wave of the Human Electric and Magnetic Response to Sound: A Review and an Analysis of the Component Structure. Psychophysiology, 24(4), 375-425. https://doi.org/10.1111/j.1469-8986.1987.tb00311.x

Näpflin, M., Wildi, M., \& Sarnthein, J. (2007). Test-retest reliability of resting EEG spectra validates a statistical signature of persons. Clinical Neurophysiology, 118(11), 2519-2524. https://doi.org/10.1016/j.clinph.2007.07.022 
Narushima, K., McCormick, L. M., Yamada, T., Thatcher, R. W., \& Robinson, R. G. (2010). Subgenual cingulate theta activity predicts treatment response of repetitive transcranial magnetic stimulation in participants with vascular depression. Journal of Neuropsychiatry and Clinical Neurosciences, 22(1), 75-84. https://doi.org/10.1176/jnp.2010.22.1.75

Neuper, C., \& Pfurtscheller, G. (2001). Event-related dynamics of cortical rhythms: frequencyspecific features and functional correlates. International Journal of Psychophysiology: Official Journal of the International Organization of Psychophysiology, 43(1), 41-58. https://doi.org/10.1016/s0167-8760(01)00178-7

Nierenberg, A. A., Gray, S. M., \& Grandin, L. D. (2001). Mood disorders and suicide. The Journal of Clinical Psychiatry, 62 Suppl 2, 27-30.

Nock, M. K., Hwang, I., Sampson, N. A., \& Kessler, R. C. (2010). Mental disorders, comorbidity and suicidal behavior: Results from the national comorbidity survey replication. Molecular Psychiatry, 15(8), 868-876. https://doi.org/10.1038/mp.2009.29

Nugent, A. C., Ballard, E. D., Gould, T. D., Park, L. T., Moaddel, R., Brutsche, N. E., \& Zarate, C. A. (2019a). Ketamine has distinct electrophysiological and behavioral effects in depressed and healthy subjects. Molecular Psychiatry, 24(7), 1040-1052. https://doi.org/10.1038/s41380-018-0028-2

Nugent, A. C., Wills, K. E., Gilbert, J. R., \& Zarate, C. A. (2019b). Synaptic potentiation and rapid antidepressant response to ketamine in treatment-resistant major depression: A replication study. Psychiatry Research - Neuroimaging, 283, 64-66. https://doi.org/10.1016/j.pscychresns.2018.09.001

Numan, T., van Dellen, E., Vleggaar, F. P., van Vlieberghe, P., Stam, C. J., \& Slooter, A. J. C. (2019). Resting State EEG Characteristics During Sedation With Midazolam or Propofol in Older Subjects. Clinical EEG and Neuroscience, 50(6), 436-443. https://doi.org/10.1177/1550059419838938

Nystrom, C., Matousek, M., \& Hallstrom, T. (1986). Relationships between EEG and clinical characteristics in major depressive disorder. Acta Psychiatrica Scandinavica, 73(4), 390 394. https://doi.org/10.1111/j.1600-0447.1986.tb02700.x

Olbrich, S., \& Arns, M. (2013). EEG biomarkers in major depressive disorder: Discriminative power and prediction of treatment response. International Review of Psychiatry, 25(5), 604618. https://doi.org/10.3109/09540261.2013.816269

Olbrich, S., Tränkner, A., Surova, G., Gevirtz, R., Gordon, E., Hegerl, U., \& Arns, M. (2016). CNS- and ANS-arousal predict response to antidepressant medication: Findings from the randomized iSPOT-D study. Journal of Psychiatric Research, 73, 108-115. https://doi.org/10.1016/j.jpsychires.2015.12.001

Olejniczak, P. (2006). Neurophysiologic basis of EEG. Journal of Clinical Neurophysiology, 23(3), 186-189. https://doi.org/10.1097/01.wnp.0000220079.61973.6c

Oranje, B., Van Berckel, B. N. M., Kemner, C., Van Ree, J. M., Kahn, R. S., \& Verbaten, M. N. (2000). The effects of a sub-anaesthetic dose of ketamine on human selective attention. Neuropsychopharmacology, 22(3), 293-302. https://doi.org/10.1016/S0893- 
133X(99)00118-9

Overall, J. E., \& Gorham, D. R. (1962). The Brief Psychiatric Rating Scale. Psychological Reports, 10(3), 799-812. https://doi.org/10.2466/pr0.1962.10.3.799

Park, Y., Jung, W., Kim, S., Jeon, H., \& Lee, S. H. (2019). Frontal alpha asymmetry correlates with suicidal behavior in major depressive disorder. Clinical Psychopharmacology and Neuroscience, 17(3), 377-387. https://doi.org/10.9758/cpn.2019.17.3.377

Pascual-Marqui, R. D., Lehmann, D., Koukkou, M., Kochi, K., Anderer, P., Saletu, B., Tanaka, H., Hirata, K., John, E. R., Prichep, L., Biscay-Lirio, R., \& Kinoshita, T. (2011). Assessing interactions in the brain with exact low-resolution electromagnetic tomography. Philosophical Transactions of the Royal Society A: Mathematical, Physical and Engineering Sciences, 369(1952), 3768-3784. https://doi.org/10.1098/rsta.2011.0081

Peltoniemi, M. A., Saari, T. I., Hagelberg, N. M., Laine, K., Neuvonen, P. J., \& Olkkola, K. T. (2012). S-ketamine concentrations are greatly increased by grapefruit juice. European Journal of Clinical Pharmacology, 68(6), 979-986. https://doi.org/10.1007/s00228-0121214-9

Penn, E., \& Tracy, D. K. (2012). The drugs don't work? antidepressants and the current and future pharmacological management of depression. Therapeutic Advances in Psychopharmacology, 2(5), 179-188. https://doi.org/10.1177/2045125312445469

Phillips, J. L., Norris, S., Talbot, J., Birmingham, M., Hatchard, T., Ortiz, A., Owoeye, O., Batten, L. A., \& Blier, P. (2019). Single, repeated, and maintenance ketamine infusions for treatment-resistant depression: A randomized controlled trial. American Journal of Psychiatry, 176(5), 401-409. https://doi.org/10.1176/appi.ajp.2018.18070834

Pizzagalli, D. A. (2011). Frontocingulate dysfunction in depression: Toward biomarkers of treatment response. Neuropsychopharmacology, 36(1), 183-206. https://doi.org/10.1038/npp.2010.166

Pizzagalli, D. A., Oakes, T. R., \& Davidson, R. J. (2003). Coupling of theta activity and glucose metabolism in the human rostral anterior cingulate cortex: An EEG/PET study of normal and depressed subjects. Psychophysiology, 40(6), 939-949. https://doi.org/10.1111/14698986.00112

Pizzagalli, D. A., Oakes, T. R., Fox, A. S., Chung, M. K., Larson, C. L., Abercrombie, H. C., Schaefer, S. M., Benca, R. M., \& Davidson, R. J. (2004). Functional but not structural subgenual prefrontal cortex abnormalities in melancholia. Molecular Psychiatry, 9(4), 325. https://doi.org/10.1038/sj.mp.4001501

Pizzagalli, D. A., Webb, C. A., Dillon, D. G., Tenke, C. E., Kayser, J., Goer, F., Fava, M., McGrath, P., Weissman, M., Parsey, R., Adams, P., Trombello, J., Cooper, C., Deldin, P., Oquendo, M. A., McInnis, M. G., Carmody, T., Bruder, G., \& Trivedi, M. H. (2018). Pretreatment rostral anterior cingulate cortex theta activity in relation to symptom improvement in depression: A randomized clinical trial. JAMA Psychiatry, 75(6), 547-554. https://doi.org/10.1001/jamapsychiatry.2018.0252

Pizzagalli, D., Pascual-Marqui, R. D., Nitschke, J. B., Oakes, T. R., Larson, C. L., Abercrombie, 
H. C., Schaefer, S. M., Koger, J. V., Benca, R. M., \& Davidson, R. J. (2001). Anterior cingulate activity as a predictor of degree of treatment response in major depression: Evidence from brain electrical tomography analysis. American Journal of Psychiatry, 158(3), 405-415. https://doi.org/10.1176/appi.ajp.158.3.405

Popa, L., Dragos, H., Pantelemon, C., Verisezan Rosu, O., \& Strilciuc, S. (2020). The Role of Quantitative EEG in the Diagnosis of Neuropsychiatric Disorders. In Journal of medicine and life (Vol. 13, Issue 1, pp. 8-15). NLM (Medline). https://doi.org/10.25122/jml-20190085

Porcelli, S., Drago, A., Fabbri, C., Gibiino, S., Calati, R., \& Serretti, A. (2011). Pharmacogenetics of antidepressant response. Journal of Psychiatry and Neuroscience, 36(2), 87-113. https://doi.org/10.1503/jpn.100059

Qiao, Z., Yu, Y., Wang, L., Yang, X., Qiu, X., Zhang, C., Ning, N., Shi, J., Chen, L., Li, Z., Liu, J., Xu, J., Zhao, L., \& Yang, Y. (2013). Impaired pre-attentive change detection in major depressive disorder patients revealed by auditory mismatch negativity. Psychiatry Research - Neuroimaging, 211(1), 78-84. https://doi.org/10.1016/j.pscychresns.2012.07.006

Restuccia, D., Vollono, C., Scaloni, L., Buccelletti, F., \& Camardese, G. (2016). Abnormality of Auditory Mismatch Negativity in Depression and Its Dependence on Stimulus Intensity. Clinical EEG and Neuroscience, 47(2), 105-112. https://doi.org/10.1177/1550059415584704

Ricardo-Garcell, J., González-Olvera, J. J., Miranda, E., Harmony, T., Reyes, E., Almeida, L., Galán, L., Díaz, D., Ramírez, L., Fernández-Bouzas, A., \& Aubert, E. (2009). EEG sources in a group of patients with major depressive disorders. International Journal of Psychophysiology, 71(1), 70-74. https://doi.org/10.1016/j.ijpsycho.2008.07.021

Rinne, T., Alho, K., Ilmoniemi, R. J., Virtanen, J., \& Näätänen, R. (2000). Separate time behaviors of the temporal and frontal mismatch negativity sources. NeuroImage, 12(1), 14 19. https://doi.org/10.1006/nimg.2000.0591

Rivolta, D., Heidegger, T., Scheller, B., Sauer, A., Schaum, M., Birkner, K., Singer, W., Wibral, M., \& Uhlhaas, P. J. (2015). Ketamine dysregulates the amplitude and connectivity of highfrequency oscillations in cortical-subcortical networks in humans: Evidence from restingstate magnetoencephalography-recordings. Schizophrenia Bulletin, 41(5), 1105-1114. https://doi.org/10.1093/schbul/sbv051

Roh, S. C., Kim, J. S., Kim, S., Kim, Y., \& Lee, S. H. (2020). Frontal alpha asymmetry moderated by suicidal ideation in patients with major depressive disorder: A comparison with healthy individuals. Clinical Psychopharmacology and Neuroscience, 18(1), 58-66. https://doi.org/10.9758/CPN.2020.18.1.58

Rosburg, T., \& Kreitschmann-Andermahr, I. (2016). The effects of ketamine on the mismatch negativity (MMN) in humans - A meta-analysis. Clinical Neurophysiology, 127(2), 13871394. https://doi.org/10.1016/j.clinph.2015.10.062

Rosburg, T., Marinou, V., Haueisen, J., Smesny, S., \& Sauer, H. (2004). Effects of lorazepam on the neuromagnetic mismatch negativity $(\mathrm{MMNm})$ and auditory evoked field component N100m. Neuropsychopharmacology, 29(9), 1723-1733. 
https://doi.org/10.1038/sj.npp.1300477

Roser, P., Haussleiter, I. S., Chong, H. J., Maier, C., Kawohl, W., Norra, C., \& Juckel, G. (2011). Inhibition of cerebral type 1 cannabinoid receptors is associated with impaired auditory mismatch negativity generation in the ketamine model of schizophrenia. Psychopharmacology, 218(4), 611-620. https://doi.org/10.1007/s00213-011-2352-y

Rush, A. J. (2007). The varied clinical presentations of major depressive disorder. The Journal of Clinical Psychiatry, 68 Suppl 8, 4-10.

Rush, A. J., Trivedi, M. H., Wisniewski, S. R., Nierenberg, A. A., Stewart, J. W., Warden, D., Niederehe, G., Thase, M. E., Lavori, P. W., Lebowitz, B. D., McGrath, P. J., Rosenbaum, J. F., Sackeim, H. A., Kupfer, D. J., Luther, J., \& Fava, M. (2006). Acute and longer-term outcomes in depressed outpatients requiring one or several treatment steps: A STAR*D report. American Journal of Psychiatry, 163(11), 1905-1917. https://doi.org/10.1176/ajp.2006.163.11.1905

Sackeim, H. A. (2001). The definition and meaning of treatment-resistant depression. The Journal of Clinical Psychiatry, 62 Suppl 1, 10-17.

Sampaio, I., Puga, F., Veiga, H., Cagy, M., Piedade, R., \& Ribeiro, P. (2008). The influence of bromazepam on cortical power distribution. Anais Da Academia Brasileira de Ciencias, 80(2), 335-340. https://doi.org/10.1590/S0001-37652008000200012

Sanacora, G., Smith, M. A., Pathak, S., Su, H. L., Boeijinga, P. H., McCarthy, D. J., \& Quirk, M. C. (2014). Lanicemine: A low-trapping NMDA channel blocker produces sustained antidepressant efficacy with minimal psychotomimetic adverse effects. Molecular Psychiatry, 19(9), 978-985. https://doi.org/10.1038/mp.2013.130

Sanacora, G., Treccani, G., \& Popoli, M. (2012). Towards a glutamate hypothesis of depression: An emerging frontier of neuropsychopharmacology for mood disorders.

Neuropharmacology, 62(1), 63-77. https://doi.org/10.1016/j.neuropharm.2011.07.036

Schiller, M. J. (2019). Quantitative electroencephalography in guiding treatment of major depression. Frontiers in Psychiatry, 10(JAN). https://doi.org/10.3389/fpsyt.2018.00779

Schmidt, F. M., Sander, C., Dietz, M.-E., Nowak, C., Schröder, T., Mergl, R., Schönknecht, P., Himmerich, H., \& Hegerl, U. (2017). Brain arousal regulation as response predictor for antidepressant therapy in major depression. Scientific Reports, 7, 45187.

https://doi.org/10.1038/srep45187

Schomer, D. L., \& da Silva, F. (2010). Niedermeyer's Electroencephalography: Basic Principles, Clinical Applications, and Related Fields. In Niedermeyer's Electroencephalography. Wolters Kluwer Health.

Seeck, M., Lazeyras, F., Michel, C. M., Blanke, O., Gericke, C. A., Ives, J., Delavelle, J., Golay, X., Haenggeli, C. A., De Tribolet, N., \& Landis, T. (1998). Non-invasive epileptic focus localization using EEG-triggered functional MRI and electromagnetic tomography. Electroencephalography and Clinical Neurophysiology, 106(6), 508-512. https://doi.org/10.1016/S0013-4694(98)00017-0

Shaw, A. D., Saxena, N., Jackson, L. E., Hall, J. E., Singh, K. D., \& Muthukumaraswamy, S. D. 
(2015). Ketamine amplifies induced gamma frequency oscillations in the human cerebral cortex. European Neuropsychopharmacology, 25(8), 1136-1146.

https://doi.org/10.1016/j.euroneuro.2015.04.012

Sheehan. (1998). The Mini-International Neuropsychiatric Interview (M.I.N.I.): The

Development and Validation of a Structured Diagnostic Psychiatric Interview for DSM-IV and ICD-10. In J Clin Psychiatry (Vol. 59, Issue suppl 20). Physicians Postgraduate Press, Inc.

Shiroma, P. R., Johns, B., Kuskowski, M., Wels, J., Thuras, P., Albott, C. S., \& Lim, K. O. (2014). Augmentation of response and remission to serial intravenous subanesthetic ketamine in treatment resistant depression. Journal of Affective Disorders, 155(1), 123-129. https://doi.org/10.1016/j.jad.2013.10.036

Singh, J. B., Fedgchin, M., Daly, E. J., De Boer, P., Cooper, K., Lim, P., Pinter, C., Murrough, J. W., Sanacora, G., Shelton, R. C., Kurian, B., Winokur, A., Fava, M., Manji, H., Drevets, W. C., \& Van Nueten, L. (2016). A double-blind, randomized, placebo-controlled, dosefrequency study of intravenous ketamine in patients with treatment-resistant depression. American Journal of Psychiatry, 173(8), 816-826. https://doi.org/10.1176/appi.ajp.2016.16010037

Smit, D. J. A., Posthuma, D., Boomsma, D. I., \& De Geus, E. J. C. (2005). Heritability of background EEG across the power spectrum. Psychophysiology, 42(6), 691-697. https://doi.org/10.1111/j.1469-8986.2005.00352.x

Smith, K. (2014). Mental health: a world of depression. In Nature (Vol. 515, Issue 7526, p. 181). Nature. https://doi.org/10.1038/515180a

Spronk, D., Arns, M., Barnett, K. J., Cooper, N. J., \& Gordon, E. (2011). An investigation of EEG, genetic and cognitive markers of treatment response to antidepressant medication in patients with major depressive disorder: A pilot study. Journal of Affective Disorders, 128(1-2), 41-48. https://doi.org/10.1016/j.jad.2010.06.021

Stone, J., Kotoula, V., Dietrich, C., De Simoni, S., Krystal, J. H., \& Mehta, M. A. (2015). Perceptual distortions and delusional thinking following ketamine administration are related to increased pharmacological MRI signal changes in the parietal lobe. Journal of Psychopharmacology, 29(9), 1025-1028. https://doi.org/10.1177/0269881115592337

Sumner, R. L., McMillan, R., Spriggs, M. J., Campbell, D., Malpas, G., Maxwell, E., Deng, C., Hay, J., Ponton, R., Sundram, F., \& Muthukumaraswamy, S. D. (2020). Ketamine improves short-term plasticity in depression by enhancing sensitivity to prediction errors. European Neuropsychopharmacology, 38. https://doi.org/10.1016/j.euroneuro.2020.07.009

Takei, Y., Kumano, S., Hattori, S., Uehara, T., Kawakubo, Y., Kasai, K., Fukuda, M., \& Mikuni, M. (2009). Preattentive dysfunction in major depression: A magnetoencephalography study using auditory mismatch negativity. Psychophysiology, 46(1), 52-61.

https://doi.org/10.1111/j.1469-8986.2008.00748.x

Tallon-Baudry, C., Bertrand, O., Delpuech, C., \& Pernier, J. (1996). Stimulus specificity of phase-locked and non-phase-locked $40 \mathrm{~Hz}$ visual responses in human. Journal of Neuroscience, 16(13), 4240-4249. https://doi.org/10.1523/jneurosci.16-13-04240.1996 
Tenke, C. E., Kayser, J., Alvarenga, J. E., Abraham, K. S., Warner, V., Talati, A., Weissman, M. M., \& Bruder, G. E. (2018). Temporal stability of posterior EEG alpha over twelve years. Clinical Neurophysiology, 129(7), 1410-1417. https://doi.org/10.1016/j.clinph.2018.03.037

Tenke, C. E., Kayser, J., Manna, C. G., Fekri, S., Kroppmann, C. J., Schaller, J. D., Alschuler, D. M., Stewart, J. W., McGrath, P. J., \& Bruder, G. E. (2011). Current source density measures of electroencephalographic alpha predict antidepressant treatment response. Biological Psychiatry, 70(4), 388-394. https://doi.org/10.1016/j.biopsych.2011.02.016

Thatcher, R. W. (2010). Validity and reliability of quantitative electroencephalography. Journal of Neurotherapy, 14(2), 122-152. https://doi.org/10.1080/10874201003773500

Thibodeau, R., Jorgensen, R. S., \& Kim, S. (2006). Depression, anxiety, and resting frontal EEG asymmetry: A meta-analytic review. Journal of Abnormal Psychology, 115(4), 715-729. https://doi.org/10.1037/0021-843X.115.4.715

Trevethan, R. (2017). Sensitivity, Specificity, and Predictive Values: Foundations, Pliabilities, and Pitfalls in Research and Practice. Frontiers in Public Health, 5, 307. https://doi.org/10.3389/fpubh.2017.00307

Trullas, R., \& Skolnick, P. (1990). Functional antagonists at the NMDA receptor complex exhibit antidepressant actions. European Journal of Pharmacology, 185(1), 1-10. https://doi.org/10.1016/0014-2999(90)90204-J

Umbricht, D., Schmid, L., Koller, R., Vollenweider, F. X., Hell, D., \& Javitt, D. C. (2000). Ketamine-induced deficits in auditory and visual context-dependent processing in healthy volunteers: Implications for models of cognitive deficits in schizophrenia. Archives of General Psychiatry, 57(12), 1139-1147. https://doi.org/10.1001/archpsyc.57.12.1139

van der Vinne, N., Vollebregt, M. A., van Putten, M. J. A. M., \& Arns, M. (2017). Frontal alpha asymmetry as a diagnostic marker in depression: Fact or fiction? A meta-analysis. NeuroImage: Clinical, 16, 79-87. https://doi.org/10.1016/j.nicl.2017.07.006

van der Vinne, N., Vollebregt, M. A., van Putten, M. J. A. M., \& Arns, M. (2019). Stability of frontal alpha asymmetry in depressed patients during antidepressant treatment. NeuroImage: Clinical, 24. https://doi.org/10.1016/j.nicl.2019.102056

Van Diepen, R. M., Foxe, J. J., \& Mazaheri, A. (2019). The functional role of alpha-band activity in attentional processing: the current zeitgeist and future outlook. Current Opinion in Psychology, 29, 229-238. https://doi.org/10.1016/j.copsyc.2019.03.015

Vande Voort, J. L., Morgan, R. J., Kung, S., Rasmussen, K. G., Rico, J., Palmer, B. A., Schak, K. M., Tye, S. J., Ritter, M. J., Frye, M. A., \& Bobo, W. V. (2016). Continuation phase intravenous ketamine in adults with treatment-resistant depression. Journal of Affective Disorders, 206, 300-304. https://doi.org/10.1016/j.jad.2016.09.008

Veselis, R. A., Reinsel, R., Sommer, S., \& Carlon, G. (1991). Use of neural network analysis to classify electroencephalographic patterns against depth of midazolam sedation in intensive care unit patients. Journal of Clinical Monitoring, 7(3), 259-267. https://doi.org/10.1007/BF01619271

Vitacco, D., Brandeis, D., Pascual-Marqui, R., \& Martin, E. (2002). Correspondence of event- 
related potential tomography and functional magnetic resonance imaging during language processing. Human Brain Mapping, 17(1), 4-12. https://doi.org/10.1002/hbm.10038

Vlisides, P. E., Bel-Bahar, T., Lee, U. C., Li, D., Kim, H., Janke, E., Tarnal, V., Pichurko, A. B., McKinney, A. M., Kunkler, B. S., Picton, P., \& Mashour, G. A. (2017). Neurophysiologic Correlates of Ketamine Sedation and Anesthesia: A High-density Electroencephalography Study in Healthy Volunteers. Anesthesiology, 127(1), 58-69. https://doi.org/10.1097/ALN.0000000000001671

Vlisides, P. E., Bel-Bahar, T., Nelson, A., Chilton, K., Smith, E., Janke, E., Tarnal, V., Picton, P., Harris, R. E., \& Mashour, G. A. (2018). Subanaesthetic ketamine and altered states of consciousness in humans. British Journal of Anaesthesia, 121(1), 249-259. https://doi.org/10.1016/j.bja.2018.03.011

Waberski, T. D., Kreitschmann-Andermahr, I., Kawohl, W., Darvas, F., Ryang, Y., Gobbelé, R., \& Buchner, H. (2001). Spatio-temporal source imaging reveals subcomponents of the human auditory mismatch negativity in the cingulum and right inferior temporal gyrus. Neuroscience Letters, 308(2), 107-110. https://doi.org/10.1016/S0304-3940(01)01988-7

Wade, E. C., \& Iosifescu, D. V. (2016). Using Electroencephalography for Treatment Guidance in Major Depressive Disorder. In Biological Psychiatry: Cognitive Neuroscience and Neuroimaging. https://doi.org/10.1016/j.bpsc.2016.06.002

Wagner, S., Engel, A., Engelmann, J., Herzog, D., Dreimüller, N., Müller, M. B., Tadić, A., \& Lieb, K. (2017). Early improvement as a resilience signal predicting later remission to antidepressant treatment in patients with Major Depressive Disorder: Systematic review and meta-analysis. Journal of Psychiatric Research, 94, 96-106. https://doi.org/10.1016/j.jpsychires.2017.07.003

Wei, Y., Chang, L., \& Hashimoto, K. (2020). A historical review of antidepressant effects of ketamine and its enantiomers. Pharmacology Biochemistry and Behavior, 190. https://doi.org/10.1016/j.pbb.2020.172870

Widge, A. S., Bilge, M. T., Montana, R., Chang, W., Rodriguez, C. I., Deckersbach, T., Carpenter, L. L., Kalin, N. H., \& Nemeroff, C. B. (2019). Electroencephalographic biomarkers for treatment response prediction in major depressive illness: A meta-analysis. American Journal of Psychiatry, 176(1), 44-56. https://doi.org/10.1176/appi.ajp.2018.17121358

Wilkinson, S. T., Ballard, E. D., Bloch, M. H., Mathew, S. J., Murrough, J. W., Feder, A., Sos, P., Wang, G., Zarate, C. A., \& Sanacora, G. (2018a). The effect of a single dose of intravenous ketamine on suicidal ideation: A systematic review and individual participant data meta-analysis. American Journal of Psychiatry, 175(2), 150-158. https://doi.org/10.1176/appi.ajp.2017.17040472

Wilkinson, S. T., Farmer, C., Ballard, E. D., Mathew, S. J., Grunebaum, M. F., Murrough, J. W., Sos, P., Wang, G., Gueorguieva, R., \& Zarate, C. A. (2019). Impact of midazolam vs. saline on effect size estimates in controlled trials of ketamine as a rapid-acting antidepressant. Neuropsychopharmacology, 44(7), 1233-1238. https://doi.org/10.1038/s41386-019-0317-8

Wilkinson, S. T., Katz, R. B., Toprak, M., Webler, R., Ostroff, R. B., \& Sanacora, G. (2018b). 
Acute and longer-term outcomes using ketamine as a clinical treatment at the yale psychiatric hospital. Journal of Clinical Psychiatry, 79(4).

https://doi.org/10.4088/JCP.17m11731

Winkler, I. (2007). Interpreting the mismatch negativity. Journal of Psychophysiology, 21(3-4), 147-163. https://doi.org/10.1027/0269-8803.21.34.147

Wong, J. J., O’Daly, O., Mehta, M. A., Young, A. H., \& Stone, J. M. (2016). Ketamine modulates subgenual cingulate connectivity with the memory-related neural circuit-a mechanism of relevance to resistant depression? PeerJ, 2016(2).

https://doi.org/10.7717/peerj.1710

World Health Organization. (2017). Depression and Other Common Mental Disorders Global Health Estimates.

Worrell, G. A., Lagerlund, T. D., Sharbrough, F. W., Brinkmann, B. H., Busacker, N. E., Cicora, K. M., \& O'Brien, T. J. (2000). Localization of the epileptic focus by low-resolution electromagnetic tomography in patients with a lesion demonstrated by MRI. Brain Topography, 12(4), 273-282. https://doi.org/10.1023/A:1023407521772

Zanos, P., Moaddel, R., Morris, P. J., Georgiou, P., Fischell, J., Elmer, G. I., Alkondon, M., Yuan, P., Pribut, H. J., Singh, N. S., Dossou, K. S. S., Fang, Y., Huang, X. P., Mayo, C. L., Wainer, I. W., Albuquerque, E. X., Thompson, S. M., Thomas, C. J., Zarate, C. A., \& Gould, T. D. (2016). NMDAR inhibition-independent antidepressant actions of ketamine metabolites. Nature, 533(7604), 481-486. https://doi.org/10.1038/nature17998

Zarate, C. A., Singh, J. B., Carlson, P. J., Brutsche, N. E., Ameli, R., Luckenbaugh, D. A., Charney, D. S., \& Manji, H. K. (2006). A randomized trial of an N-methyl-D-aspartate antagonist in treatment-resistant major depression. Archives of General Psychiatry, 63(8), 856-864. https://doi.org/10.1001/archpsyc.63.8.856

Zoon, H. F. A., Veth, C. P. M., Arns, M., Drinkenburg, W. H. I. M., Talloen, W., Peeters, P. J., \& Kenemans, J. L. (2013). EEG alpha power as an intermediate measure between brainderived neurotrophic factor Val66Met and depression severity in patients with major depressive disorder. Journal of Clinical Neurophysiology, 30(3), 261-267.

https://doi.org/10.1097/WNP.0b013e3182933d6e 
Appendix I: Effects of Ketamine on Resting-State EEG Activity and Their Relationship to Perceptual/Dissociative Symptoms in Healthy Humans (Published in Frontiers in Pharmacology: Neuropharmacology

\section{OPEN ACCESS}

Edited by:

Andrew C. McCreary,

Janssen Prevention Center,

Netherlands

Reviewed by:

Charles H. Large,

Autifony Therapeutics Limited, Italy

Luigia Trabace,

University of Foggia, Italy

Pim Drinkenburg,

Janssen Pharmaceutica, Belgium

${ }^{*}$ Correspondence:

Verner Knott

verner.knott@theroyal.ca

Specialty section:

This article was submitted to

Neuropharmacology,

a section of the journal

Frontiers in Pharmacology

Received: 24 May 2016

Accepted: 15 September 2016

Published: 27 September 2016

Citation:

de la Salle S, Choueiry J, Shah D, Bowers H, Mclntosh J, llivitsky $V$ and Knott V (2016) Effects of Ketamine on

Resting-State EEG Activity and Their Relationship

to Perceptual/Dissociative Symptoms

in Healthy Humans.

Front. Pharmacol. 7:348

doi: 10.3389/fphar.2016.00348

\section{Effects of Ketamine on Resting-State EEG Activity and Their Relationship to Perceptual/Dissociative Symptoms in Healthy Humans}

\author{
Sara de la Salle1, Joelle Choueiry ${ }^{2}$, Dhrasti Shah ${ }^{1}$, Hayley Bowers ${ }^{3}$, Judy Mclntosh ${ }^{4}$, \\ Vadim Ilivitsky ${ }^{5,6}$ and Verner Knott ${ }^{1,2,4,5 *}$ \\ 'School of Psychology, University of Ottawa, Ottawa, ON, Canada, ${ }^{2}$ Department of Cellular and Molecular Medicine, \\ University of Ottawa, Ottawa, ON, Canada, ${ }^{3}$ Department of Psychology, University of Guelph, Guelph, ON, Canada, \\ ${ }^{4}$ University of Ottawa Institute of Mental Health Research, Ottawa, ON, Canada, ${ }^{5}$ Department of Psychiatry, University of \\ Ottawa, Ottawa, ON, Canada, ${ }^{6}$ Royal Ottawa Mental Health Centre, Ottawa, ON, Canada
}

N-methyl-D-aspartate (NMDA) receptor antagonists administered to healthy humans results in schizophrenia-like symptoms, which preclinical research suggests are due to glutamatergically altered brain oscillations. Here, we examined resting-state electroencephalographic activity in 21 healthy volunteers assessed in a placebocontrolled, double-blind, randomized study involving administration of either a saline infusion or a sub-anesthetic dose of ketamine, an NMDA receptor antagonist. Frequency-specific current source density (CSD) was assessed at sensor-level and source-level using eLORETA within regions of interest of a triple network model of schizophrenia (this model posits a dysfunctional switching between large-scale Default Mode and Central Executive networks by the monitor-controlling Salience Network). These CSDs were measured in each session along with subjective symptoms as indexed with the Clinician Administered Dissociative States Scale. Ketamine-induced CSD reductions in slow (delta/theta and alpha) and increases in fast (gamma) frequencies at scalp electrode sites were paralleled by frequency-specific CSD changes in the Default Mode, Central Executive, and Salience networks. Subjective symptoms scores were increased with ketamine and ratings of depersonalization in particular were associated with alpha CSD reductions in general and in specific regions of interest in each of the three networks. These results tentatively support the hypothesis that pathological brain oscillations associated with hypofunctional NMDA receptor activity may contribute to the emergence of the perceptual/dissociate symptoms of schizophrenia.

Keywords: ketamine, $N$-methyl-D-aspartate, brain oscillations, electroencephalography, psychosis, schizophrenia

\section{INTRODUCTION}

Blockade of glutamate $N$-methyl-D-aspartate receptors (NMDAR) by a subanesthetic dose of ketamine in humans transiently induces negative, positive and cognitive symptoms similar to those found in schizophrenia (SZ). Together with findings in animals of behavioral, biochemical, and electrophysiological alterations with NMDAR antagonists, convergent genetic, molecular and 
neuroimaging evidence points to NMDAR hypofunction in SZ (Javitt, 2012; Javitt et al., 2012). This supports use of the ketamine challenge as a pharmacological approach to generating insights into SZ symptoms and their underlying pathophysiologic processes (Frohlich and Van Horn, 2014).

Oscillatory brain activity is considered a fundamental process in the temporal coordination of circuits linked to perceptual, cognitive, and emotional functions. This brain activity, as measured by the amplitude/power of electroencephalographic (EEG) rhythms, is increasingly seen as an indicator of intrinsic brain function when assessed during stimulation/task-free states (Spencer, 2014). Further, this activity has been found to be altered in SZ and to vary with symptomatology (Boutros et al., 2008, 2014; Moran and Hong, 2011; Galderisi et al., 2014). Aberrant "resting-state" spectral EEG profiles in psychosis have invariably shown increased activity in low frequency (delta, theta) EEG and magnetoencephalographic (MEG) rhythms (Fehr et al., 2003; Boutros et al., 2008; Venables et al., 2009; Uhlhaas and Singer, 2010; Moran and Hong, 2011; Ranlund et al., 2014) which have been found specifically evidenced in chronic SZ patients (Sponheim et al., 1994, 2000; Narayanan et al., 2014), their firstdegree relatives (Narayanan et al., 2014) and first-episode SZ patients (Sponheim et al., 2000), but not in individuals at-risk for psychosis (Ranlund et al., 2014). Spontaneous alpha activity, which predominates in healthy individual's EEG, is significantly diminished in chronic SZ patients (though unaffected in some studies), with varying findings that depend on scalp region, and whether slower or faster frequencies are examined within this band (Sponheim et al., 1994, 2000; Knyazeva et al., 2008; Hong et al., 2012; Narayanan et al., 2014; Goldstein et al., 2015; Kim et al., 2015). Similar inconsistencies are evident with beta rhythms, with chronic patients showing either no abnormalities (Wada et al., 1994; Kam et al., 2013; Kim et al., 2015) or increased activity (Sponheim et al., 1994; Venables et al., 2009; Narayanan et al., 2014). As with delta/theta findings (Galderisi et al., 2009), these inconsistencies can be partially attributed to the effects of chronicity, medication and patient symptomatology (Czobor and Volovka, 1992; Merlo et al., 1998; Knott et al., 2001; Cerdán et al., 2005; Venables et al., 2009; Moran and Hong, 2011). The recent interest in resting gamma activity, increased in some (Czobor and Volovka, 1992; Merlo et al., 1998; Knott et al., 2001; Cerdán et al., 2005; Kam et al., 2013; Kocsis et al., 2013; Tikka et al., 2013, 2014, 2015) but not all EEG or MEG studies (Sponheim et al., 1994) of SZ patients (Rutter et al., 2009; Fuggetta et al., 2014; Hirano et al., 2015) is of particular interest both because of its purported role in feature binding and coordination of local neuronal populations, and because these rhythms are in part dependent on the activity of Parvalbumin-containing GABAergic cortical inhibitory interneurons, which are diminished in SZ patients and subject to NMDAR modulation from excitatory pyramidal cells (Lee et al., 2003; Herrmann and Dermirlap, 2005; Sun et al., 2011; Gandal et al., 2012; Lewis et al., 2012; Cohen et al., 2015). Integrative brain functions, however, are modulated through multiple oscillatory processes in different frequency bands, each with differing neuropharmacological sensitivities. As such, the study of the full frequency spectrum and its pharmacological basis is critical for deciphering the complex electrophysiologic abnormalities in SZ patients (Moran and Hong, 2011).

Electrophysiological recordings in animals with NMDAR antagonists such as ketamine and MK-801 recreate some of the background spontaneous EEG features of SZ (Hunt and Kasicki, 2013). Consistent with patient findings, increases in delta are dose-dependent and evident in cortical and subcortical (hippocampus, thalamus) regions. Theta frequency activity also shows these dose related changes, with power in this band consistently increased in cortex but reduced in the hippocampus. Ketamine's effects on these rhythms in rodents, although generally augmenting beta and gamma by its actions on PVinterneurons in multiple cortical regions (Pinault, 2008; Carlén et al., 2012), vary depending on dose, acute vs chronic treatments, subcortical region and within-band (slow vs. fast) frequencies (Pinault, 2008; Roopun et al., 2008; Hakami et al., 2009; Carlén et al., 2012; Kittelberger et al., 2012; Hunt and Kasicki, 2013; Kocsis et al., 2013). In humans administered relatively high (anesthetic) doses of ketamine ( $2 \mathrm{mg} / \mathrm{kg})$, activity is increased in theta and beta bands and decreased in alpha (Schultz et al., 1990; Bojak et al., 2013). Sub-anesthetic psychotomimetic bolus doses of ketamine $(0.2-0.5 \mathrm{mg} / \mathrm{kg})$ have also increased theta and decreased alpha in baseline (pre-stimulus) EEGs (Kochs et al., 1996). In the only two human resting-state EEG studies to date, reductions in delta, alpha, and beta (gamma not assessed) were found with an ultra-low ketamine dose (Knott et al., 2006), and increases in gamma (other bands not assessed) were observed with a psychotogenic dose, but relationships with induced dissociative or psychotic-like symptoms were not examined (Sanacora et al., 2014). Of the two human resting MEG studies, subanesthetic ketamine increased gamma in both while reducing either alpha (Muthukumaraswamy et al., 2015) or beta (Rivolta et al., 2015).

Ketamine-modulated neuroelectric activity in rodent models suggest complex-spatiotemporal effects, which vary with frequency band and cortical area (Lee et al., 2003; Herrmann and Dermirlap, 2005; Pinault, 2008; Roopun et al., 2008; Moran and Hong, 2011; Carlén et al., 2012; Gandal et al., 2012; Lewis et al., 2012; Hunt and Kasicki, 2013; Cohen et al., 2015). Similar systematic EEG studies on healthy humans of the effects of subanesthetic, psychotomimetic doses of ketamine on the generation and cortical distribution of intrinsic brain rhythms across the frequency spectrum have not been conducted. Thus, one of our objectives has been to profile the acute effects of ketamine on the generation and topography of sensor-level EEG in low and high frequencies using reference-free current source density (CSD) measures (Nunez and Srinivasan, 2006). Such reference-free measures avoid problems associated with reference-dependent EEG indices (Kayser and Teake, 2010); reference-independent CSD measures reduce volume conduction from distal sites, sharpen spatial resolution, and are more closely related to neuronal activity and the strength of underlying current generators (Nicholson, 1973; Nunez and Srinivasan, 2006; Kayser and Teake, 2010).

A second objective has been the evaluation of the effects of NMDAR blockade on source-level, electric activity within Nekovarova et al.'s (2014) recent triple network (TN) model 
of SZ psychopathology. Largely investigated with fMRI (Fox and Raichle, 2007), but also assessed with PET (Raichle et al., 2001) and EEG (Chen et al., 2008; Yuan et al., 2012), brain regions have been shown to be dynamically organized into functional networks of intercorrelated areas (or "nodes") that act together to perform specific tasks (Bressler, 1995). Of the full repertoire of brain networks, all of which are continuously and dynamically "active" not only during cognition but even when at "rest" (Smith et al., 2009), during sleep (Fukunaga et al., 2006) and under anesthesia (Vincent et al., 2007), there are three large-scale domain-specific networks that make up the model which exhibits abnormalities in SZ (Bressler and Menon, 2010) and depression (Mulders et al., 2015): the default mode network (DMN), the central executive network (CEN), and the salience network (SN). This TN model relies on accumulating neuroimaging evidence in healthy humans indicating that SN, which is involved in the orientating of attention to the most homeostatically relevant (salient) of ongoing extrapersonal (sensory) and intrapersonal (limbic driven) events (Bressler and Menon, 2010), causally influences anticorrelated activation of DMN and CEN. Clinical and cognitive symptoms of SZ are in part attributed to aberrant switching by the $\mathrm{SN}$ between internal processes (i.e., self-referential, autobiographical functions) supported by the DMN and external processes (i.e., attention and processing of exogenous stimuli) supported by the CEN as a result of a dysfunctional SN, a system that enables the switch between various dynamic brain states (Sridharan et al., 2008; Palaniyappan et al., 2012).

Adopting a region of interest (ROI) approach with exact low resolution brain electromagnetic tomography (i.e., eLORETA), which is an electrophysiological neuroimaging method that allows a reliable source localization of surface level electrical signals (Pascual-Marqui, 2011; Pascual-Marqui et al., 2011), we assessed ketamine-induced changes in two key regional nodes anchoring each of the three large-scale networks: DMN [ventromedial prefrontal cortex (VMPFC) and posterior cingulate cortex (PCC)]; CEN [dorsolateral prefrontal cortex (DLPFC) and posterior parietal cortex (PPC)]; and SN [anterior cingulate cortex (ACC) and anterior insula (AI)]. Based on limited human EEG studies with NMDAR antagonists, we hypothesized that relative to placebo, ketamine administration in healthy humans would produce SZ-like scalp surface CSD changes ( $\uparrow$ delta, beta and gamma, and $\downarrow$ alpha). Ketamine has been shown to alter resting $\mathrm{SN}$ and DMN function (Bonhomme et al., 2016) and given the pivotal role of the $\mathrm{SN}$ in the TN model and the available neuroimaging evidence indicating a primary role of dopamine in the interaction of the SN with subcortical sites, whilst the within-network activity of the SN and its interaction with other large-scale networks is thought to predominantly depend on glutamate/gammaaminobutyric acid (GABA) neurotransmission (Palaniyappan et al., 2012), we expected ketamine to result in band-dependent CSD alterations within the cortical networks. We further hypothesized that these sensor- and source-level EEG changes would be accompanied by dissociative symptoms characteristic of SZ.

\section{MATERIALS AND METHODS}

\section{Participants}

A sample of 21 right-hand dominant, male volunteers (mean age $=21.3$ years, $\pm 2.5 \mathrm{SE}$ ) were recruited via local media advertisements. Male volunteers were chosen in order to avoid any potential confounding effects of menstrually related hormonal changes on ketamine response. Volunteers underwent both a psychiatric interview, using both the Structured Clinical Interview, non-Patient version (SCID-NP) for DMS-IV (Williams et al., 1992) and the Family Interview for Genetic Studies (FIGS), (Maxwell, 1992) and a medical exam, including electrocardiogram and routine blood/urine laboratory tests as well as using toxicology for drug use. Only healthy individuals who were medication-free, non-smokers (smoked $<100$ cigarettes, none in the past year), reported no neurological disease, and had no personal or family (first-degree biological relatives) psychiatric/substance abuse history were included in the study. The protocol was approved by the Research Ethics Board of the Royal Ottawa Health Care Group and all participants provided written informed consent. This study was conducted in accordance with the Tri-Council Policy Statement on Ethical Conduct for Research Involving Humans. Participants were compensated \$75 CAD for each of the two test sessions.

\section{Design}

Volunteers participated in the test sessions within a randomized, double-blind design in which half were administered placebo in the first session and ketamine in the second session, while the remaining half received treatments in the reverse order. A minimum 5 days interval separated the two test sessions.

\section{Procedures}

Test sessions (beginning 8:00 a.m.) followed overnight abstinence from food, caffeine, alcohol, and drugs and began with insertion of an antecubital intravenous line after which, participants rested for a 45-min adaptation period and EEG electrodes were positioned on the scalp. A racemic ketamine or saline $0.90 \% \mathrm{w} / \mathrm{v}$ of $\mathrm{NaCl}$ bolus dose was then administered $(0.26 \mathrm{mg} / \mathrm{kg})$ over $10 \mathrm{~min}$ and was immediately followed by a constant infusion of $0.65 \mathrm{mg} / \mathrm{kg}$ lasting $\sim 60 \mathrm{~min}$, as per Krystal et al. (1999). Beginning $10 \mathrm{~min}$ after the initiation of the constant infusion, a 3 min EEG recording was collected, after which participants were evaluated with the Clinician Administered Dissociative States Scale (CADSS), (Bremner et al., 1998) which has been previously shown to be sensitive to ketamine administration (Krystal et al., 1994). The scale consists of 19 self-report items, (scored as $0=$ not at all; $1=$ slightly; $2=$ moderately; $3=$ considerably; 4 = extremely), which yielded three subjective subscale scores (amnesia, depersonalization, and derealisation) and it also has similarly scored 8 clinician rated items that result in one observer rated score.

\section{EEG Recording}

Electroencephalographic activity was recorded according to recommended pharmaco-EEG standards (Knott, 2000; Saletu et al., 2006; Jobert et al., 2012) while participants were seated 
reclined with eyes closed. Using an electronically linked mastoid reference, Brain Vision ${ }^{\circledR}$ Recorder software Version 2 (Brain Products, Munich, Germany) was used to sample $(1000 \mathrm{~Hz})$ electrical activity from 28 EEG and 2 (vertical and horizontal) electrooculograpic (EOG) channels, with amplifiers/filters set at $0.1-100 \mathrm{~Hz}$, and with electrode impedance $<5 \mathrm{k} \Omega$. The electrodes were divided into nine regions, including left frontal (Fp1, F7, F3) middle frontal (Fz, FC1, FC2), right frontal (Fp2, F4, F8), left temporal-central (T7, FC5, CP5), middle temporalcentral (C3, Cz, C4), right temporal-central (T8, FC6, CP6), left posterior (P7, P3, O1), middle posterior $(\mathrm{Pz}, \mathrm{CP} 1, \mathrm{CP} 2)$, right posterior $(\mathrm{P} 4, \mathrm{P} 8, \mathrm{O} 2)$.

\section{Scalp Surface CSD Analysis}

Off-line processing with Brain Vision ${ }^{\otimes}$ Analyzer 2 software (Brain Products, Munich, Germany) involved: visual inspection of recordings for elimination of activity with prominent ocular/muscle/cardiac contamination or drowsiness (i.e., alpha suppression combined with increased slow waves), ocular correction of EEG with an algorithm (Gratton et al., 1983); automatic rejection of activity with voltages $>100 \mathrm{uV}$ and finally application of independent component analysis (ICA) to remove residual ocular (e.g., microsaccades) artifacts (McMenamin et al., 2010). The resulting artifact-free recordings (minimum $120 \mathrm{~s}$ ) were transformed into reference-free CSD estimates using a spherical Laplacian algorithm (Nunez et al., 1997; Mima and Hallett, 1999). CSD estimates were computed using the fourth order spherical spline interpolation, and a maximal degree of Legendre polynomials of 10 (Perrin et al., 1989). Corrected, non-overlapping 2-s epochs were subjected to a Fast Fourier Transform algorithm (using a Hanning window with $10 \%$ taper length) for computation of absolute CSD (averaged across epochs at each electrode site) in delta $(1-4 \mathrm{~Hz})$, theta $(4-8 \mathrm{~Hz})$, alpha $(8-12.5 \mathrm{~Hz})$, beta $(12.5-30 \mathrm{~Hz})$, and gamma $(30-60 \mathrm{~Hz})$ bands. A natural log transform was applied to computed CSD values (Gasser et al., 1982) and individual electrodes were aggregated to create an average CSD value for anterior, temporal-central, and posterior regions.

\section{Source-Localized CSD Analysis}

eLORETA (version 2081104) was used to compute the intracortical source distribution of the electric activity from the surface EEG data (Pascual-Marqui, 2011; Pascual-Marqui et al., 2011). eLORETA is a weighted minimum non-linear inverse solution method applied to EEG recordings for computation of three dimensional distribution of electric cortical activity with zero location error (Pascual-Marqui, 2011; Pascual-Marqui et al., 2011). Localization with this methodology, even with a lower number of electrodes used in this study has been cross-validated with functional and structural MRI, PET and intracranial recordings. Relying on the Montreal Neurologic Institute average MRI brain (MNI 152) (Canuet et al., 2011) and a solution space restricted to cortical gray matter/hippocampus, eLORETA analysis of each EEG epoch results in current density being computed at each of 6239 cortical voxels ( $5 \mathrm{~mm}$ spatial resolution) for each of the frequency bands. Results were averaged across epochs for each individual and frequency band in placebo and ketamine sessions. Defined ROIs were based on definitions of the Brodmann Areas (BA) provided by eLORETA software package, which are based on the Talairach Daemon ${ }^{1}$. A single voxel (at the centroid of each BA) was used for each ROI due to eLORETA's restricted spatial resolution, which makes it unable to separate two closely spaced sources, and additionally, the single centroid voxel (the closest to the center of the BA mass) is an excellent representative of the corresponding BA. The BAs comprising the representative hubs of the three targeted networks within the TN model included: BA 11 (VMPFC), BA 23/30 (PCC), BA 9 (DLPFC), BA 40 (PPC), BA 24/32 (ACC), and BA 47 (AI). CSDs were derived for left and right hemisphere of each BA.

\section{Statistics}

Statistical analysis was conducted in SPSS 23 (SPSS Inc., Chicago, IL, USA). For each frequency band, scalp CSD values were analyzed with a separate repeated measures analysis of variance (ANOVA) involving treatment (placebo, ketamine), region (anterior, temporal-central, posterior) and laterality (left, middle, right) factors. Separate repeated measures ANOVAs were also conducted for each band-indexed network and involved treatment, hemisphere (left, right) and network hub (two levels) factors. Significant $(p<0.05)$ Greenhouse-Geisser estimates were followed up with Bonferroni adjusted $T$-test comparisons. The three primary rating measures, depersonalization, derealisation and observation, evidenced non-normal distributions and were analyzed by the non-parametric Wilcoxon Signed Ranks Test (WSRT). Relationships were examined with ketamine-induced CSD and CADSS difference scores, obtained by subtracting values in the placebo session from values in the ketamine session, and analysis with the non-parametric Spearman's correlation coefficient. For source-localized EEG, these CADSSCSD relationships were separately examined for each hub of each network. For scalp EEG, changes in CADSS were examined in relation to the CSD averaged across the ketamine affected electrode sites.

\section{RESULTS}

\section{CADSS Rating Scores}

The mean $( \pm S E)$ values for the rating scores for the placebo and ketamine conditions are displayed in Figure 1. Significant increased self-ratings for amnesia (WSRT $=-2.83 \mathrm{df}=1 / 40$, $p<0.005$ ), depersonalization (WSRT $=-3.53 \mathrm{df}=1 / 40$, $p<0.0001$ ), and derealisation symptoms (WSRT $=-3.36$, $\mathrm{df}=1 / 40, p<0.01)$ were shown for the ketamine compared to placebo infusion condition. Ketamine also increased the observer rated symptom scores (WSRT $=-3.72, \mathrm{df}=1 / 40, p<0.0001$ ) relative to placebo.

\section{Scalp Surface CSD}

As shown in Figure 2, scalp analyzed current density values for slow and fast oscillations were significantly influenced by

\footnotetext{
${ }^{1}$ http://talairach.org/
} 


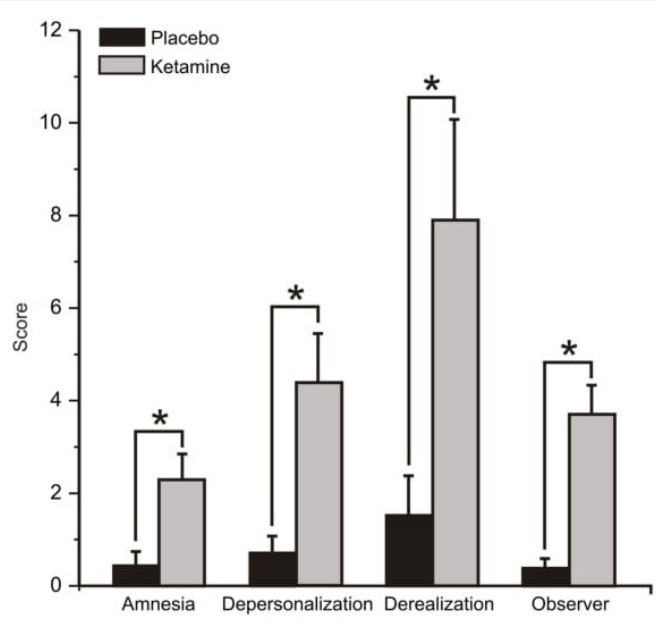

FIGURE 1 | Mean ( \pm SE) Clinician Administered Dissociative States Scale (CADSS) values for the rating scores for the placebo and ketamine conditions $(\boldsymbol{n}=\mathbf{2 1}) .{ }^{*} p<0.05$.

ketamine. Delta exhibited significant treatment $(F=6.14$, $\mathrm{df}=1 / 20, p<0.02)$ and treatment $\times$ region interaction effects $(F=3.52, \mathrm{df}=2 / 40, p<0.04)$, with ketamine acting to reduce CSD bilaterally in temporal-central $(p<0.02)$ and posterior $(p<0.01)$ regions. Within a treatment region $\times$ laterality interaction $(F=3.77, \mathrm{df}=4 / 80, p<0.007)$, theta CSD was similarly reduced in the left $(p<0.04)$ and right $(p<0.03)$ posterior cortex. Exhibiting treatment $(F=9.91$, df $=1 / 40$, $p<0.005)$, treatment $\mathrm{x}$ region $(F=4.43, \mathrm{df}=2 / 40$, $p<0.02)$ and treatment $\times$ region $\times$ laterality interaction effects $(F=4.35, \mathrm{df}=4 / 80, p<0.003)$, alpha current density was significantly diminished in left $(p<0.002)$ and right $(p<0.01)$ anterior, middle $(p<0.006)$ and right $(p<0.004)$ temporal-central, and posterior regions following ketamine infusion. Beta current density was not affected by ketamine treatment but significant treatment effects for gamma $(F=5.49$, $\mathrm{df}=1 / 20, p<0.03$ ) showed that ketamine increased current density of these oscillations across all scalp regions. Table 1 displays a summary of the regional effects of ketamine vs. placebo.

\section{Source-Localized CSD Default Mode Network (DMN)}

Significant ketamine effects on ROIs of the DMN are shown in Figure 3. Analysis of delta yielded a significant treatment $\times$ hemisphere interaction $(F=6.93 \mathrm{df}=1 / 20$, $p<0.007$ ), with ketamine (vs. placebo) acting to reduce CSD in the right hemisphere of the VMPFC and PCC $(p<0.05)$. A significant treatment effect $(F=5.46$, $\mathrm{df}=1 / 20, p<0.03)$ showed general ketamine-induced reductions in theta CSD across both hubs of the DMN. Within treatment $(F=12.49, \mathrm{df}=1 / 20, p<0.004)$ and treatment $\times$ hub interaction effects $(F=5.58, \mathrm{df}=1 / 20$, $p<0.03)$, ketamine was found to diminish alpha CSD in the PCC $(p<0.002)$.

In contrast with the other oscillatory bands, gamma CSD was significantly increased by ketamine in both the VMPFC and PCC hubs of the DMN $(F=5.72$, df $=1 / 20$, $p<0.03)$.

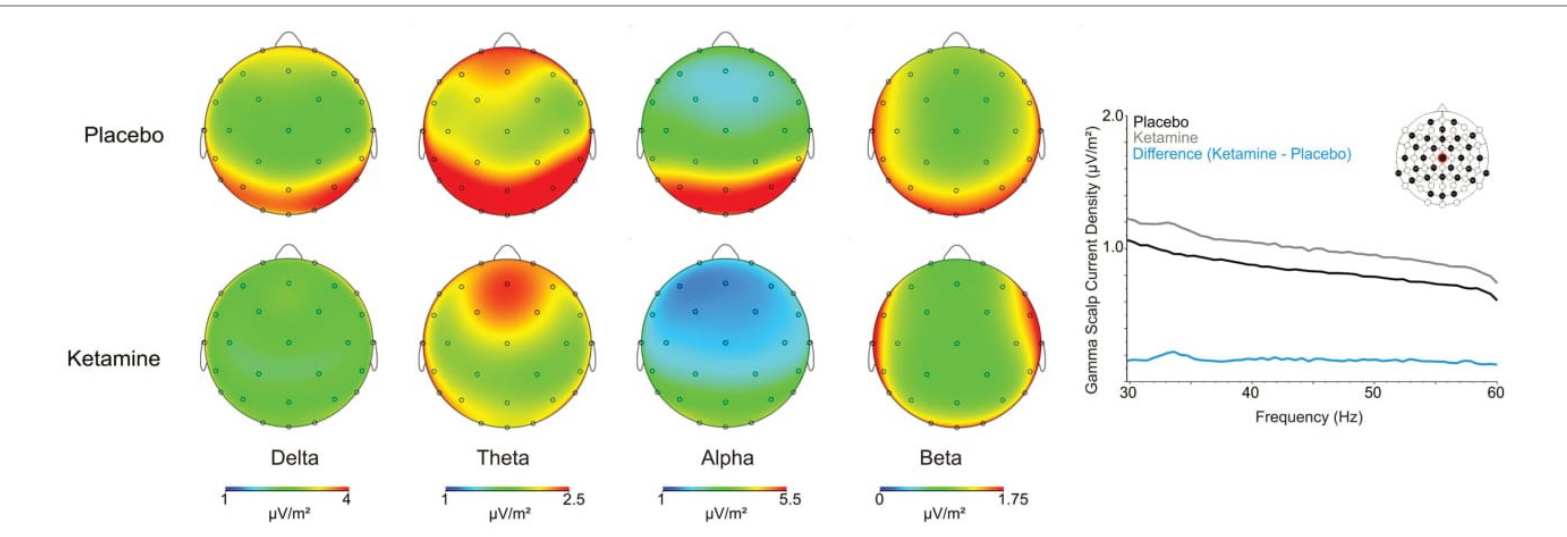

FIGURE 2 | Grand averaged scalp current source density (CSD) topographic maps for the placebo and ketamine conditions for the frequency bands, together with a spectral graph of gamma from the $\mathrm{Cz}$ site.

TABLE 1 | Summary of significant ketamine-induced scalp surface current source density (CSD) regional changes associated with each frequency band.

\begin{tabular}{|c|c|c|c|c|}
\hline Delta & Theta & Alpha & Beta & Gamma \\
\hline \multirow{3}{*}{$\begin{array}{l}\downarrow \text { Left, Right temporal-central } \\
\downarrow \text { Left, Right Posterior }\end{array}$} & $\downarrow$ Left, Right Posterior & $\downarrow$ Left, Right Anterior & No changes & $\uparrow$ Left, Middle, Right Anterior \\
\hline & & $\downarrow$ Middle, Right temporal-central & & $\uparrow$ Left, Middle, Right temporal-central \\
\hline & & $\downarrow$ Left, Middle, Right Posterior & & $\uparrow$ Left, Middle, Right Posterior \\
\hline
\end{tabular}




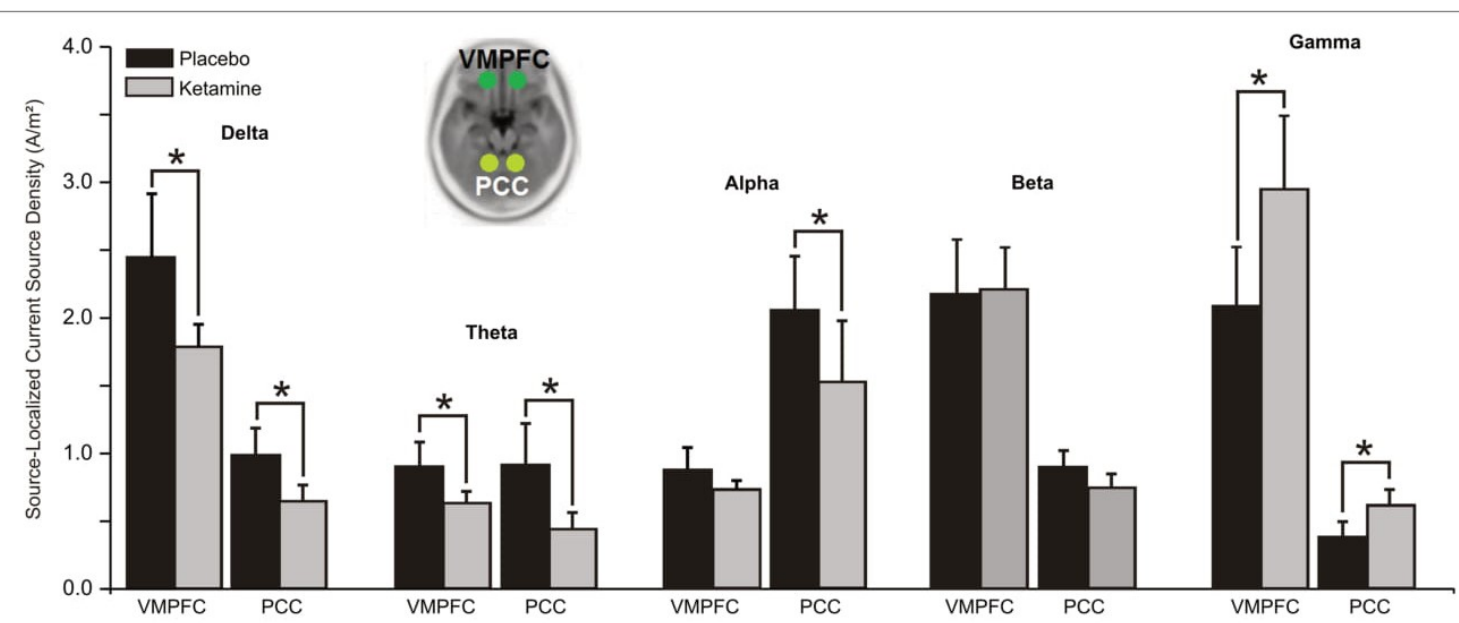

FIGURE 3 | Mean ( \pm SE) non-logged source-localized CSD values $\left(A / \mathrm{m}^{2}\right)$ for the two regions of interest of the default mode network $(n=21)$. $* p<0.05$. VMPFC, ventromedial prefrontal cortex; PCC, posterior cingulate cortex.

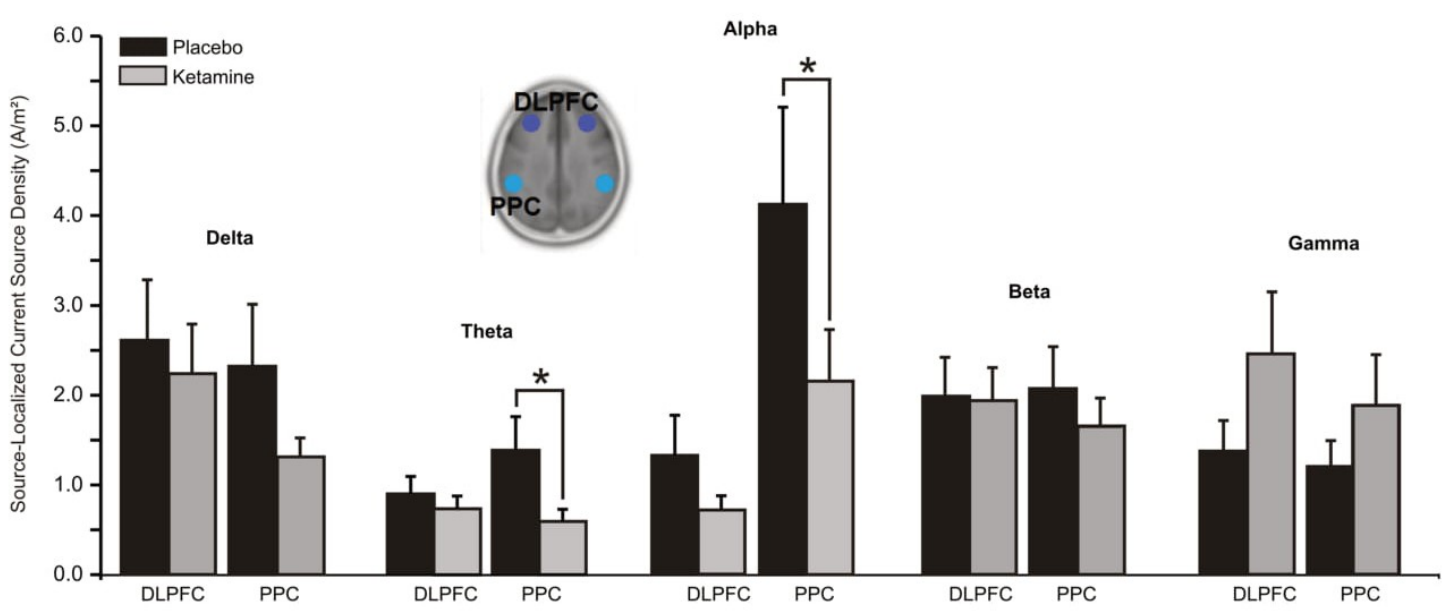

FIGURE 4 | Mean $( \pm S E)$ non-logged source-localized CSD values $\left(A / \mathrm{m}^{2}\right)$ for the two regions of interest of the central executive network $(n=21)$. ${ }^{*} p<0.05$. DLPFC, dorsolateral prefrontal cortex; PPC, posterior parietal cortex.

\section{Central Executive Network (CEN)}

For both theta $(F=7.21, \mathrm{df}=1 / 20, p<0.01)$ and alpha $(F=6.03$, $\mathrm{df}=1 / 20, p<0.02)$, significant treatment $\times$ region interactions saw the CSD of these oscillations in the PPC to be reduced by ketamine compared to placebo (Figure 4). No significant treatment effects were observed for delta, beta or gamma CSD.

\section{Salience Network (SN)}

Whereas a significant overall treatment effect $(F=7.96$, $\mathrm{df}=1 / 20, p<0.01$ ) showed alpha CSD to be reduced in both $\mathrm{ACC}$ and $\mathrm{AI}$ hubs of the $\mathrm{SN}$, a treatment $\mathrm{x}$ region interaction evidenced with beta $(F=10.47 \mathrm{df}=1 / 20, p<0.004)$ found current density reductions to be limited to the ACC $(p<0.05)$. Ketamine, by contrast, significantly $(F=4.85$, df $=1 / 20$, $p<0.04)$ increased ACC and AI gamma current density (Figure 5).

\section{CADSS-CSD Relationships}

Changes in symptoms induced by ketamine were significantly associated with ketamine-induced changes in scalp and sourcelocalized EEG, but only with alpha CSD (Figure 6). Scalp EEG alpha changes were negatively correlated with depersonalization ratings $(r=-0.58, p<0.006)$. For source-localized EEG, reductions in alpha CSD in the left $(r=-0.66, p<0.04)$ and right $(r=-0.64, p<0.002)$ PCC hemispheres of the DMN, and the right PPC $(r=-0.54, p<0.01)$ and $\mathrm{AI}$ hemispheres $(r=-0.45, p<0.04)$ of the CEN and SN, respectively, were related to increased depersonalization rating scores. No other 


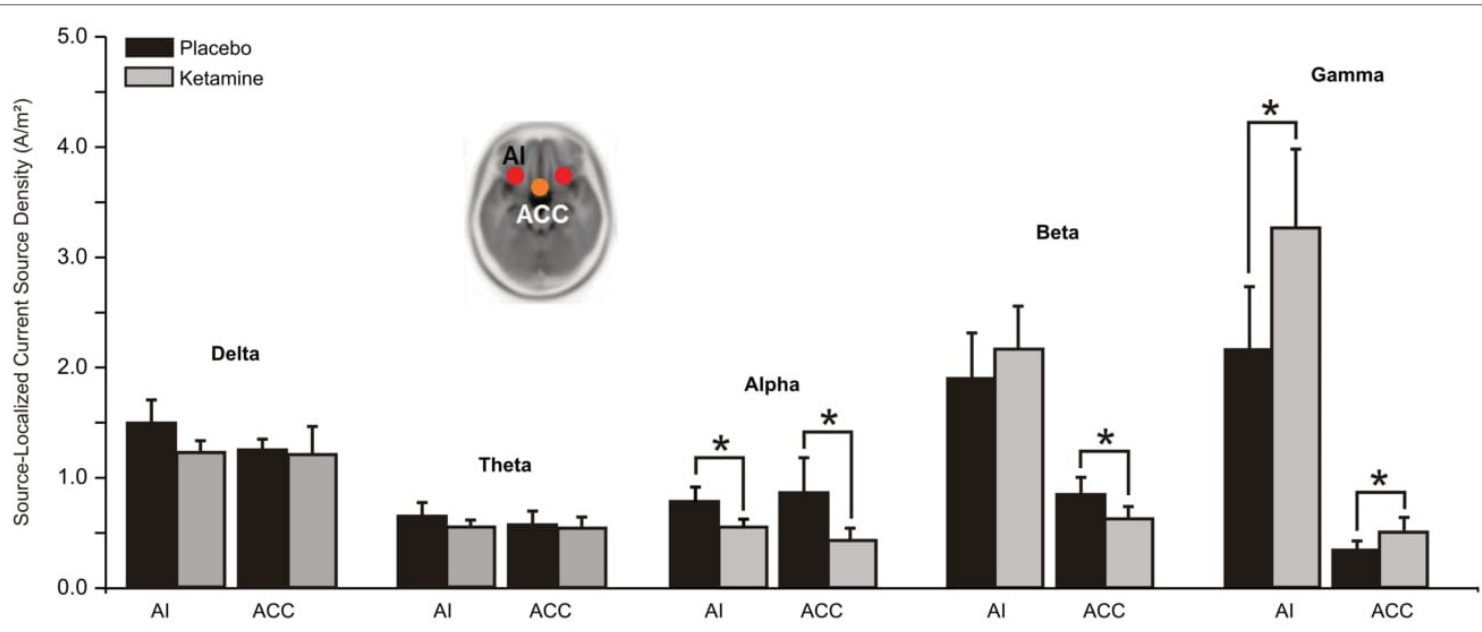

FIGURE 5 | Mean ( \pm SE) non-logged source-localized CSD values $\left(A / \mathrm{m}^{2}\right)$ for the two regions of interest of the salience network $(n=21) .{ }^{*} p<0.05$. $\mathrm{Al}$, anterior insula; ACC, anterior cingulate cortex.

correlations were evidences with delta, theta, beta, or gamma rhythms.

\section{DISCUSSION}

Although there are plenty of single blind EEG/MEG studies, this is the first randomized, placebo-controlled, double-blind crossover study of healthy humans administered a sub-anesthetic, psychotomimetic dose of ketamine that demonstrates changes in resting-state EEG across the frequency spectrum. The work builds on extensive animal research on the electrophysiological effects of ketamine and well-studied EEG correlates of anesthetic doses of ketamine. As key findings, we report CSD alterations in low and high frequency rhythms analyzed from scalp EEG recordings and these were accompanied by similar current density changes in source-localized cortical ROIs within the three large-scale networks of the schizophrenia model, namely the DMN, CEN and SN. These electrocortical effects of ketamine were paralleled by changes in dissociative states, the degree of which was significantly correlated with scalp and SZ network alpha current density changes.

$\mathrm{N}$-methyl-D-aspartate receptor antagonism in preclinical models has most frequently been associated with increases in low (delta/theta) and high (beta/gamma) frequencies (Hunt and Kasicki, 2013). These in vivo electrocortical findings with NMDA receptor blockade, paralleled to some extent by oscillatory changes in in vitro (Kim et al., 2015) recordings and intracortical networks (Hakami et al., 2009), vary considerably, particularly with respect to theta rhythms, which have also been shown to be reduced both with acute and chronic ketamine treatment (Kittelberger et al., 2012). Our EEG observations in healthy volunteers of increased gamma and reduced non-gamma activity with a ketamine dose typically used to model SZ in part confirm previous independent study findings of EEG alpha and gamma changes seen with anesthetic and sub-anesthetic dosing in humans (Schultz et al., 1990; Kochs et al., 1996; Knott et al., 2006; Bojak et al., 2013; Sanacora et al., 2014) and they correspond with ketamine-induced alpha/beta and gamma alterations in recent resting MEG studies (Muthukumaraswamy et al., 2015; Rivolta et al., 2015). These emerging electrocortical patterns with NMDA receptor blockade seen in our study and in other investigations are reminiscent of some of the reports of aberrant EEG activity in SZ. Deficits in resting spontaneous alpha band activity reported across the clinical course of the disease (chronic SZ, first episode psychosis, prodromal SZ, relatives of SZ probrands) (Boutros et al., 2008; Goldstein et al., 2015; Kim et al., 2015) were mimicked in our EEG recordings across scalp regions following NMDA antagonist treatment. They were also seen in recent MEG recordings (Muthukumaraswamy et al., 2015), and were concurrent with diffuse increments in gamma also described in some recent resting state studies of SZ (Kam et al., 2013; Kocsis et al., 2013; Tikka et al., 2013, 2014, 2015). These parallel observations with alpha and gamma spectral frequencies contrast with the response of delta-theta and beta activities to NMDA receptor blockade as the reduction in current density with ketamine, particularly in the low frequencies, is contrary to the observation that increased local and global EEG delta/theta is one of the more consistent findings reported in unmedicated, first episode, and chronic SZ patients (Boutros et al., 2008; Moran and Hong, 2011; Kim et al., 2015).

Thus, acute NMDA receptor hypofunction may mediate some of the EEG disturbances associated with psychosis, particularly those relating to high frequency gamma perturbations, which in this study likely result from ketamine inhibition of NMDA receptors on GABA interneurons and disinhibition of pyramidal neurons (Grunze et al., 1996; Maccaferri and Dingledine, 2002; Jackson et al., 2004; Homayoun and Moghaddam, 2007) subpopulations, some of which act to increase cortical gamma during inhibition (Lovett-Barron et al., 2012; Royer et al., 


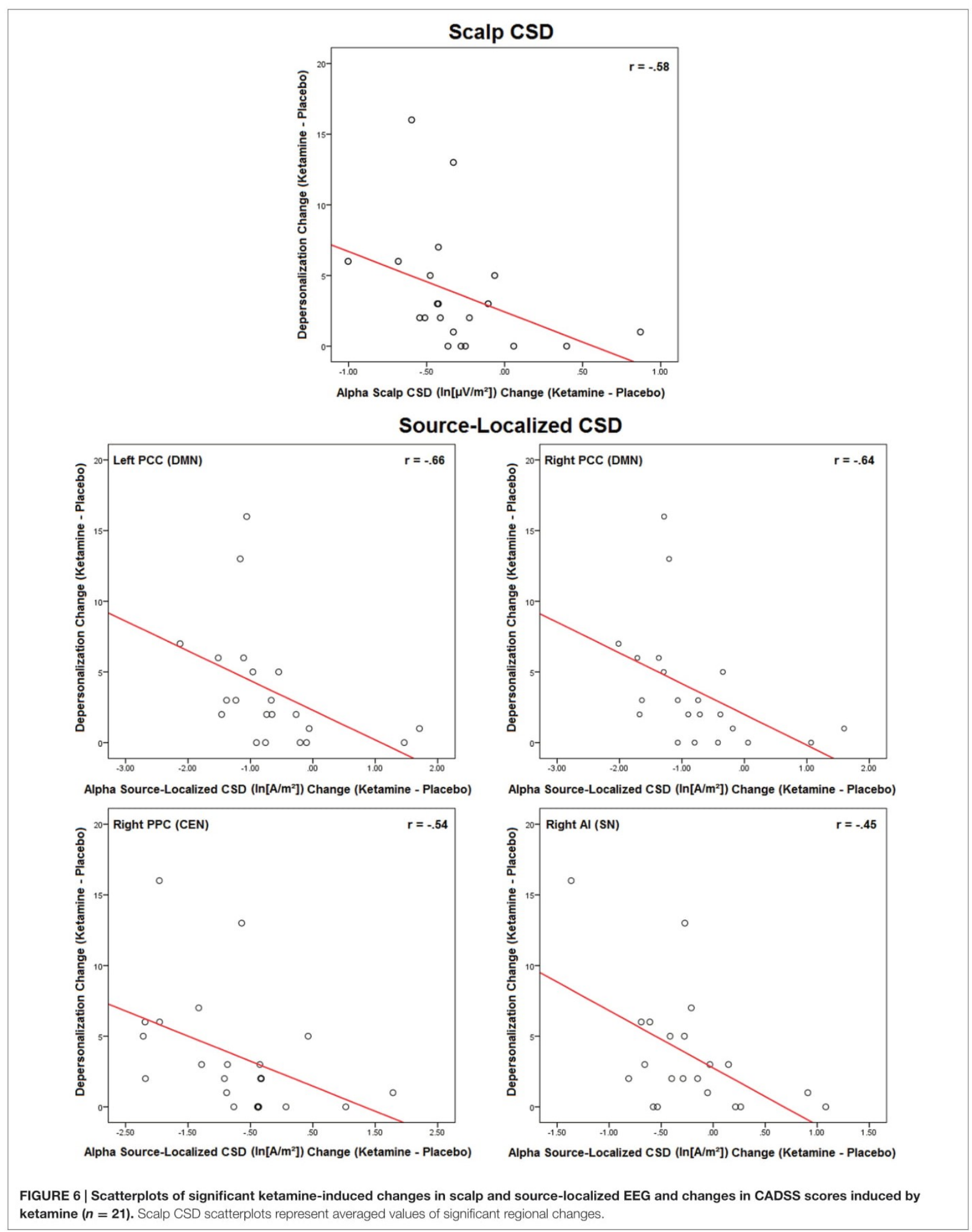


2012). Considering ketamine's multitude of effects, with actions at muscarinic, opioid, and adrenergic receptors and actions blocking serotonin and norepinephrine transporters (Bergman, 1999; Chen et al., 2009; Stahl, 2013), any one or more of these mechanisms [and/or the purported increase in non-NMDA glutamate (i.e., AMPA and kainate) receptor neurotransmission resulting from release of GABA restraint during impaired NMDA receptor function (Olney and Farber, 1995; Moghaddam et al., 1997)] may underly changes in non-gamma rhythms seen in SZ and with acute NMDA receptor blockade. Given that oxidative stress is thought to play a crucial role in several brain disorders including psychosis (Sorce and Krause, 2009; Schiavone et al., 2012, 2016), is involved in the modulation of neuronal activity (Infanger et al., 2006), and is increased with acute subanesthetic doses of ketamine and controls the resultant prefrontal glutamate release accompanying NMDA receptor antagonism (Sorce et al., 2010), brain oxidative stress may be one novel mechanism underlying the SZ-like electrophysiological changes with ketamine administration.

Contributing cortical sources to our observed ketamine modulated EEG profile were examined within the TN model of SZ. Although there is increasing interest in the pathophysiology of brain networks in SZ, to our knowledge this is the first human study to explore CSD in nodes of three well defined large-scale neural networks during acute NMDA receptor hypofunction. Directional changes in surface-level EEG current density induced by ketamine within each frequency band were mirrored by CSD alterations across the networks and, whereas only with alpha CSD were all three networks shown to be affected by NMDA receptor blockade, nodes of at least one or two of the networks evidenced ketamine-induced changes in delta (DMN), theta (DMN, CEN), beta (SN), and gamma (DMN, SN) rhythms. Activity of individual high and low frequencies is associated with both overlapped and distinct sensory and cognitive functions but given the general observation that reductions in low frequency and increases in high frequency rhythms are associated with increased arousal and behavioral activation (Moran and Hong, 2011), our frequency specific oscillatory changes with ketamine generally suggest that affected nodes within the respective large scale networks are hyperactivated during NMDA antagonist treatment. The exception is seen with the ACC, which showed alpha reductions and gamma increases (activation) along with decreases in beta (suppression) during ketamine administration. This general pattern of cortical activation during waking states is very similar to that reported in healthy volunteer studies utilizing fMRI and PET to image resting cerebral blood flow and brain metabolic response to ketamine's blockade of NMDA receptors (Lahti et al., 1995; Breier et al., 1997; Vollenweider et al., 1997; Deakin et al., 2008; De Simoni et al., 2013; Doyle et al., 2013; Scherbinin et al., 2015). Although there are discrepancies in these neuroimaging studies, ketamine has typically activated frontal and temporal lobe structures as well as the AI and PCC but has suppressed the VMPFC while exhibiting mixed effects on the ACC, which has been found to be either hyperactive or hypoactive with acute antagonist treatment.

Accumulating neuroimaging evidence in healthy individuals indicates that an imbalance between the normally anti-correlated
DMN (internal, self-related processing) and CEN (external environment/task-related processing) may underlie clinical and cognitive features of various psychiatric disorders (Menon, 2011), including SZ (Northoff, 2015). Although aberrant glutamatergic signaling in either the DMN or CEN may disrupt this balance, the AI-ACC nodes of the SN network, and the AI in particular, play a critical and causal role in the switch between activation and deactivation of the two large-scale brain networks (Sridharan et al., 2008). Structural and functional abnormalities across the different stages of SZ occur in the nodes of SN (Palaniyappan et al., 2012) and dopaminergic dysfunction in the $\mathrm{SN}$ is viewed as having a central role in aberrant motivational salience (Kapur, 2003) and clinical symptoms (Palaniyappan and Liddle, 2012) in individuals with psychosis. GABA/glutamate coupling is also thought to mediate SN functions and subanesthetic doses of ketamine have been specifically shown to increase glutamate turnover in the ACC, consistent with studies in SZ in which glutamatergic levels are elevated in the ACC of early-stage, drug naïve or drug-free patients (Merritt et al., 2013; Poels et al., 2014).

Our findings of ketamine-induced increases in gamma in the ACC and AI nodes, likely resulting from reduced excitation of GABAergic interneurons and the subsequent disinhibition of glutamatergic pyramidal neurons, may represent elevated noise at pyramidal cell assemblies (Moran et al., 2015), which may act to reduce high frequency signal-to-noise ratio in response to sensory input (Saunders et al., 2012) and disrupt information processing in these networks. Given that activity in the SN typically precedes and predicts activity in both DMN and CEN (Sridharan et al., 2008), implying that the SN coordinates multi-networking activity, then alterations in gamma in the SN may act to decrease the inherent anti-correlation between the DMN and CEN, as suggested by the co-activation of these networks, which is indexed by reductions in delta/theta and alpha activity. Decreases in anti-correlation between the DMN and CEN are thought to diminish the boundaries or distinction between internally and externally oriented cognitions, confusing internal and external mental contents and resulting in "self-environment blurring" (Vollenweider et al., 1997) - thus providing a mechanistic path that may explain several core symptoms of SZ.

Oscillations generated by thalamocortical circuits are thought to be responsible for the synchronization of neural activity between different cortical regions and, depending on the frequency range of the most prominent oscillation, are associated with the appearance of specific mental states. Generally, thalamocortically generated gamma is thought to be a potential explanation for coherence of perception in the brain while alpha serves to route information to downstream regions by inhibiting neuronal processing in task-irrelevant regions (Jensen and Mazaheri, 2010; Foxe and Snyder, 2011; Klimesch, 2012), thus allowing task-relevant regions to communicate. Accordingly, the reduction in alpha and increase in gamma seen in the SN with acute ketamine may be associated with reduced salience processing capabilities (due to impaired sensory/perceptual functions) and diminished registration of salience below the threshold that is needed to allow switching between the triple networks. 
Study analysis of the subjective response to NMDA antagonist treatment assessed with CADSS showed significant dissociative effects, with ketamine infusion resulting in marked increases in amnesia, depersonalization, derealisation, and objective rater subscale scores. These dissociative phenomena, which have been consistently reported in previous ketamine studies with healthy volunteers and particularly in males (Morgan et al., 2006), are part of a spectrum of transient SZ-like psychoactive effects (including positive and negative symptoms) produced with acute subanesthetic ketamine (Krystal et al., 1994; Malhotra et al., 1996; Adler et al., 1998) and can be attenuated by inhibiting the reuptake glycine, a co-agonist at the strychnine-insensitive glycine- $\beta$ site on the NMDA receptor (D'Souza et al., 2012).

Of the perceptual effects we observed with ketamine infusion, only changes in depersonalization scores were related to the EEG alterations. Increases in depersonalization ratings were associated with reductions in alpha current density across the scalp and in specific nodes of the DMN (bilateral PCC), CEN (right PPC), and SN (right AI). Altered self-other boundary, experienced as depersonalization (feeling detached as if one's body is unreal), is one of several so-called "basic symptoms" of prodromal SZ (Maggini et al., 2002; Raballo, 2012). There is considerable overlap in midline cortical structures comprising the neural network implicated in self-specificity with those showing high resting state in the DMN, and while evidence suggests that the pregenual ACC is specifically involved in self-processing (Qin and Northoff, 2011), several neuroimaging studies have also pointed to the $\mathrm{AI}$ as being the representational cortex for the sense of self (Craig, 2002, 2009). Alpha rhythms are considered among the most important building blocks for functioning, association and communication in the brain (Basar, 2012; Basar and Guntekin, 2012; Bazanova and Vernon, 2014). Alpha is prominent in the EEG DMN profile (Chen et al., 2008), overlaps with resting-state networks identified in fMRI (Jann et al., 2007; Mantini et al., 2007), and in addition to being the main neural oscillation of self-agency (a person's feeling that his action is generated by himself; Kang et al., 2015), correlates with both perception of self-related stimuli and resting state glutamate concentration in the pregenual ACC (Bai et al., 2016). Given this context, our findings with ketamine suggest that glutamatergic modulated alpha activity may be one mechanism underlying pathological self-processing in SZ and may serve as a viable target for novel treatment interventions.

\section{Limitations}

The present results provide preliminary informative insight into the some of the electrocortical mechanisms influenced by blockade of NMDA receptors but limitations of the study must also be considered. First, ketamine is one of the most selective NMDA receptor antagonists available for human studies but it has secondary sites of action unrelated to these glutamatergic receptors and thus additional experiments with more selective NMDA receptor antagonists are needed to specifically associate our EEG findings to NMDA receptor hypofunction. Also, some of the non-specific behavioral effects associated with ketamine (e.g., drowsiness) may have compromised the study blind, and future studies may want to systematically document these behaviors to determine how these potential confounds may impact statistical analyses and data interpretation. Studies may also consider incorporating into their design an active placebo comparator drug such as midazolam, which has similar pharmacokinetic properties, rapid anesthetic and behavioral effects as ketamine. Further, acute NMDA antagonism reproduces many features of SZ but others (auditory hallucination) appear only after chronic administration (Javitt et al., 2012). The design of this study does not explicitly allow for longitudinal inferences, but different effects of short-and long-term exposure to NMDA antagonists have been demonstrated for varying neurotransmitter systems and cognitive domains (Bubeniková-Valesová et al., 2008). EEG studies with animal models of chronic ketamine users are required to profile the progression of these acute oscillatory changes. Second, EEG has relatively low spatial resolution compared to other imaging modalities such as fMRI. Future multimodal studies may wish to combine EEG recordings with concurrent fMRI or MEG recordings to further verify the sources of EEG markers. Moreover, it may be advantageous to implement ICA in the eLORETA software (eLORETA-ICA) as ICA decomposition of EEG data becomes more correct in localization and more robust to artifacts (Jonmohamadi et al., 2014) and filtered ICA time series of EEG correlates with BOLD time series in specific resting networks (Hiltunen et al., 2014). For high frequencies, which may be contaminated by muscle artifact, it would be useful to supplement this approach with electromyographic recordings and to apply canonical correlation analysis (CCA) as a blind source separation technique to remove broadband or electromyographic noise from single EEG epochs (De Clereq et al., 2006). These pre-processing strategies as well as more recently recommended state-of-the-art methodologies for analyzing high-frequency (gamma range) activity should be adopted in future ketamine-EEG research (Jobert et al., 2012; Nottage and Horder, 2015). As well, EEG assessments did not include measures of synchronization to index oscillatory coherence which may have been influenced by ketamine and may have provided insight into the pattern of aberrant regional and intra- and inter-network functional connectivity associated with NMDA receptor antagonism. Third, the dynamic TN hypothesis was not directly tested with experimental conditions that would behaviourally challenge each specific network function. Only dissociative symptoms were monitored and we did not include assessments of positive, negative, or cognitive symptoms, each of which may have been uniquely affected by ketamine infusion and differentially associated with oscillatory alterations resulting from NMDA receptor blockade. For example, beta reductions in SN ACC hub with ketamine were not associated with depersonalization ratings but, given that this oscillatory frequency is predominantly in attention tasks, its dysfunction with NMDA receptor blockade may be relevant to aberrant salience processing.

\section{CONCLUSION}

In summary, resting-state EEG appears to be a useful and efficient method for investigating the neuropharmacology of altered brain 
rhythms suspected to underlie perceptual/dissociative symptoms of SZ. In the present study, sensor-level and surface projected neural network electrocortical activity was investigated in a NMDA receptor hypofunction model of SZ and intravenous ketamine was shown to partly mimic the aberrant EEG activity observed in SZ patients and to alter oscillations in large-scale resting-state networks implicated in psychosis. Neuroelectric changes correlated with the severity of dissociative symptoms induced by NMDA receptor antagonist treatment. Together, these findings provide additional information on how the modulation of the glutamatergic system may regulate brain electric activity, supporting its potential utility both as a biomarker of NMDA receptor dysfunction and a possible target for novel treatments in psychiatric disorders such as SZ which involve glutamatergic deficits.

\section{REFERENCES}

Adler, C., Goldberg, T., Malhotra, A., Pickar, D., and Breier, A. (1998). Effects of ketamine on thought disorder, working memory and semantic memory in healthy volunteers. Biol. Psychiatry 43, 811-816. doi: 10.1016/S00063223(97)00556-8

Bai, Y., Nakao, T., Xu, J., Qin, P., Chaves, P., Heinzel, A., et al. (2016). Resting state glutamate predicts elevated pre-stimulus alpha during self-relatedness: a combined EEG-MRS study on "rest-self overlap." Soc. Neurosci. 21, 1-15. doi: $10.1080 / 17470919.2015 .1072582$

Basar, E. (2012). A review of alpha activity in integrative brain function: fundamental physiology, sensory coding, cognition and pathology. Int. J. Psychophysiol. 86, 1-24. doi: 10.1016/j.ijpsycho.2012.07.002

Basar, E., and Guntekin, B. (2012). A short review of alpha activity in cognitive processes and in cognitive impairment. Int. J. Psychophysiol. 86, 25-38. doi: 10.1016/j.ijpsycho.2012.07.001

Bazanova, O., and Vernon, D. (2014). Interpreting EEG alpha activity. Neurosci. Biobehav. Rev. 214, 94-100. doi: 10.1016/j.neubiorev.2013.05.007

Bergman, S. (1999). Ketamine: review of its pharmacology and its use in pediatric anesthesia. Anesth. Prog. 46, 10-20.

Bojak, I., Day, H., and Liley, D. (2013). Ketamine, propofol, and the EEG: a neural field analysis of HCNI-mediated interactions. Front. Comput. Neurosci. 7:22. doi: 10.3389 /fncom. 2013.00022

Bonhomme, V., Vanhaudenhuyse, A., Demertzi, A., Bruno, M. A., Jaquet, O., Bahri, M. A., et al. (2016). Resting-state network-specific breakdown of functional connectivity during ketamine alteration of consciousness in volunteers. Anesthesiology. doi: 10.1097/ALN.0000000000001275 [Epub ahead of print].

Boutros, N., Arfken, C., Galderisi, S., Warrick, J., Pratt, G., and Iacono, W. (2008) The status of spectral EEG abnormality as a diagnostic test for schizophrenia. Schizophr. Res. 99, 225-237. doi: 10.1016/j.schres.2007.11.020

Boutros, N., Mucci, A., Vignapiano, A., and Galderisi, S. (2014) Electrophysiological aberrations associated with negative symptoms in schizophrenia. Curr. Top. Behav. Neurosci. 21, 129-156. doi: 10.1007/7854_2014_303

Breier, A., Malhotra, A., Pinals, D., Weisenfeld, N. I., and Pickar, D. (1997). Association of ketamine-induced psychosis with focal activation of the prefrontal cortex in healthy volunteers. Am. J. Psychiatry 154, 805-811. doi 10.1176/ajp.154.6.805

Bremner, J., Krystal, J., Putnam, F., Southwick, S. M., Marmar, C., Charney, D. S., et al. (1998). Measurement of dissociative states with the clinicianadministered dissociative states scale (CADSS). J. Trauma. Stress 11, 125-135. doi: $10.1023 / \mathrm{A}: 1024465317902$

Bressler, S. (1995). Large-scale cortical networks and cognition. Brain Res. Brain Res. Rev. 20, 288-304. doi: 10.1016/0165-0173(94)00016-I

Bressler, S., and Menon, V. (2010). Large-scale brain networks in cognition: emerging methods and principles. Trends Cogn. Sci. 14, 277-290. doi: 10.1016/j.tics.2010.04.004

\section{AUTHOR CONTRIBUTIONS}

Each of the authors participated in this research by contributing to the conception and design of the project (VK), participant screening (SdS, JC, DS, HB, and VI), performance of the experiment (SdS, JC, DS, HB, and JM), electrophysiology and statistical analysis (SdS), and interpretation (VK and SdS).

\section{ACKNOWLEDGMENT}

Research was supported by a grant from the Natural Sciences and Engineering Research Council (NSERC) of Canada to VK (NSERC No. 210572-152799-2001).

Bubeniková-Valesová, V., Horácek, J., Vrajová, M., and Höschl, C. (2008). Models of schizophrenia in humans and animals based on inhibition of NMDA receptors. Neurosci. Biobehav. Rev. 32, 1014-1023. doi: 10.1016/j.neubiorev.2008.03.012

Canuet, L., Ishii, R., Pascual-Marquis, R., Iwase, M., Kurimoto, R., Aoki, Y. et al. (2011). Resting-state EEG source localization and functional connectivity in schizophrenia-like psychosis of epilepsy. PLoS ONE 6:e27863. doi: 10.1371/journal.pone.0027863

Carlén, M., Meletis, K., Siegle, J., Cardin, J., Futai, K., Vierling-Claassen, D., et al. (2012). A critical role for NMDA receptors in parabalbumin interneurons for gamma rhythm inhibition and behaviour. Mol. Psychiatry 17, 537-543. doi: 10.1038/mp.2011.31

Cerdán, L., Guevara, M., Sanz, A., Amezcua, C., and Ramos-Loyo, J. (2005). Brain electrical activity changes in treatment refractory schizophrenics after olanzapine treatment. Int. J. Psychophysiol. 56, 237-247. doi: 10.1016/j.ijpsycho.2004.12.008

Chen, A., Feng, W., Zhao, H., Yin, Y., and Wang, P. (2008). EEG default mode network in the human brain: spectral regional field powers. Neuroimage 41 , 561-574. doi: 10.1016/j.neuroimage.2007.12.064

Chen, X., Shu, S., and Bayliss, D. (2009). HCN1 channel subunits are a molecular substrate for hypnotic actions of ketamine. J. Neurosci. 29, 600-609. doi: 10.1523/JNEUROSCI.3481-08.2009

Cohen, S., Tsien, R., Goff, D., and Halassa, M. (2015). The impact of NMDA receptor hypofunction on GABAergic neurons in the pathophysiology of schizophrenia. Schizophr. Res. 167, 98-107. doi: 10.1016/j.schres.2014.12.026

Craig, A. (2002). How do you feel? Interoception: the sense of the physiological condition of the body. Nat. Rev. Neurosci. 8, 655-666. doi: 10.1038/nrn894

Craig, A. (2009). How do you feel now? The anterior insula and human awareness. Nat. Rev. Neurosci. 10, 59-70. doi: 10.1038/nrn2555

Czobor, P., and Volovka, J. (1992). Level of haloperidol in plasma is related to electroencephalographic findings in patients who improve. Psychiatry Res. 42, 129-144. doi: 10.1016/0165-1781(92)90077-G

De Clereq, W., Verqult, A., Vanrumite, B., Van Paesschen, W., and Van Huffel, S. (2006). Canonical correlation analysis applied to remove muscle artifacts from the electroencephalogram. IEEE Trans. Biomed. Eng. 53, 2583-2587. doi: 10.1109/TBME.2006.879459

De Simoni, S., Schwarz, A., O’Daly, O., Marquand, A. F., Brittain, C., Gonzales, C., et al. (2013). Test-retest reliability of the BOLD pharmacological MRI response to ketamine in healthy volunteers. Neuroimage 64, 75-90. doi: 10.1016/j.neuroimage.2012.09.037

Deakin, J., Lees, J., McKie, S., Hallak, J., Williams, S., and Dursun, S. (2008). Glutamate and the neural basis of the subjective effects of ketamine: a pharmaco-magnetic resonance imaging study. Arch. Gen. Psychiatry 65, 154164. doi: 10.1001/archgenpsychiatry.2007.37

Doyle, O., De Simoni, S., Schwarz, A., Brittain, C., O’Daly, O., Williams, S., et al. (2013). Quantifying the attenuation of the ketamine pharmacological magnetic resonance imaging response in humans: a validation using antipsychotic 
and glutamatergic agents. J. Pharmacol. Exp. Ther. 345, 151-160. doi: 10.1124 /jpet.112.201665

D'Souza, D., Singh, N., Elander, J., Carbuto, M., Pittman, B., Udo de Haes, J., et al. (2012). Glycine transporter inhibitor attenuates the psychotomimetic effects of ketamine in healthy males: preliminary evidence. Neuropsychopharmacology 37 , 1036-1046. doi: 10.1038/npp.2011.295

Fehr, T., Kissler, J., Wienbruch, C., Moratti, S., Elbert, T., Watzl, H., et al. (2003). Source distribution of neuromagnetic slow-wave activity in schizophrenic patients - effects of activation. Schizophr. Res. 63, 63-71. doi: 10.1016/S09209964(02)00213-X

Fox, M., and Raichle, M. (2007). Spontaneous fluctuations in brain activity observed with functional magnetic resonance imaging. Nat. Rev. Neurosci. 8, 700-711. doi: 10.1038/nrn2201

Foxe, J. J., and Snyder, A. C. (2011). The role of alpha-band brain oscillations as a sensory suppression mechanism during selective attention. Front. Psychol. 2:154. doi: $10.3389 /$ fpsyg.2011.00154

Frohlich, J., and Van Horn, J. D. (2014). Reviewing the ketamine model of schizophrenia. J. Psychopharmacol. 28, 287-302. doi: 10.1177/0269881113512909

Fuggetta, G., Bennett, M., Duke, P., and Young, A. M. (2014). Quantitative electroencephalography as a biomarker for proneness toward developing psychosis. Schizophr. Res. 153, 68-77. doi: 10.1016/j.schres.2014.01.021

Fukunaga, M., Horvitz, S., Gelderen, P., de Zwart, J., Jansma, J., Ikonomidou, V., et al. (2006). Large-amplitude, spatially correlated fluctuations in BOLD fMRI signals during rest and early sleep. Magn. Reson. Imaging 24, 979-992. doi: 10.1016/j.mri.2006.04.018

Galderisi, S., Mucci, A., Aolpe, U., and Boutros, N. (2009). Evidence-based medicine and electrophysiology in schizophrenia. Clin. EEG Neurosci. 40, 62-77. doi: 10.1177/155005940904000206

Galderisi, S., Vignapiano, A., Mucci, A., and Boutros, N. (2014). Physiological correlates of positive symptoms in schizophrenia. Curr. Top. Behav. Neurosci. 21, 103-128. doi: 10.1007/7854_2014_322

Gandal, M., Edgar, J., Klook, K., and Siegel, S. (2012). Gamma synchrony: towards a translational biomarker for the treatment-resistant symptoms of schizophrenia. Neuropharmacology 62, 1504-1518. doi: 10.1016/j.neuropharm.2011.02.007

Gasser, T., Bacher, P., and Mocks, J. (1982). Tranformations towards the normal distribution of broad band spectral parameters of the EEG. Electroencephalogr. Clin. Neurophysiol. 53, 119-124. doi: 10.1016/0013-4694(82)90112-2

Goldstein, M., Peterson, M., Saaguinetti, J., Tononi, G., and Ferrarelli, F. (2015). Topographic deficits in alpha-range resting EEG activity and steady state visual evoked responses in schizophrenia. Schizophr. Res. 168, 145-152. doi: 10.1016/j.schres.2015.06.012

Gratton, G., Coles, M., and Donchin, E. (1983). A new method for off-line removal of ocular artifact. Electroencephalogr. Clin. Neurophysiol. 55, 468-484. doi: 10.1016/0013-4694(83)90135-9

Grunze, H., Rainnie, D., Hasselmo, M., Barkai, E., Hearn, E., McCarley, R., et al. (1996). NMDA-dependent modulation of CA1 local circuit inhibition. J. Neurosci. 16, 2034-2043.

Hakami, T., Jones, N., Tolmacheva, E., Gaudias, J., Chaumont, J., Salzberg, M., et al. (2009). NMDA receptor hypofunction leads to generalized and persistent aberrant oscillations independent of hyperlocomotion and the state of consciousness. PLoS ONE 4:e6755. doi: 10.1371/journal.pone.0006755

Herrmann, C., and Dermirlap, T. (2005). Human EEG gamma oscillations in neuropsychiatric disorders. Clin. Neurophysiol. 116, 2719-2733. doi: 10.1016/j.clinph.2005.07.007

Hiltunen, T., Kantola, J., Abou-Elsoud, A., Lepola, P., Suominen, K., Starck, T., et al. (2014). Infra-slow EEG fluctuations are correlated with resting-state network dynamics in fMRI. J. Neurosci. 34, 356-362. doi: 10.1523/JNEUROSCI.027613.2014

Hirano, Y., Oribe, N., Kanba, S., Onitsuka, T., Nestor, P., and Spencer, K. (2015). Spontaneous gamma activity in schizophrenia. JAMA Psychiatry 72, 813-821. doi: 10.1001/jamapsychiatry.2014.2642

Homayoun, H., and Moghaddam, B. (2007). NMDA receptor hypofunction produces opposite effects on prefrontal cortex interneurons and pyramidal neurons. J. Neurosci. 27, 11476-11500. doi: 10.1523/JNEUROSCI.2213-07. 2007

Hong, L., Summerfelt, A., Mitchell, B., O’Donnell, P., and Thaker, G. (2012). A shared low-frequency oscillatory rhythm abnormality in resting and sensory gating in schizophrenia. Clin. Neurophysiol. 123, 285-292. doi: 10.1016/j.clinph.2011.07.025

Hunt, M., and Kasicki, S. (2013). A systematic review of the effects of NMDA receptor antagonists on oscillatory activity recorded in vivo. J. Psychopharmacol. 27, 972-986. doi: 10.1177/0269881113495117

Infanger, D. W., Sharma, R. V., and Davisson, R. L. (2006). NADPH oxidases of the brain: distribution, regulation, and function. Antioxid. Redox Signal. 28, 1583-1596. doi: 10.1089/ars.2006.8.1583

Jackson, M., Homayoun, H., and Moghaddam, B. (2004). NMDA receptor hypofunction produces concomitant firing rate potentiation and burst activity reduction in the prefrontal cortex. Proc. Natl. Acad. Sci. U.S.A. 101, 8467-8472. doi: 10.1073/pnas.0308455101

Jann, K., Dierks, T., Boesch, C., Kottlow, M., Strik, W., and Koenig, T. (2007). BOLD correlates of EEG alpha phase-locking and the fMRI default mode network. Neuroimage 45, 903-916. doi: 10.1016/j.neuroimage.2009.01.001

Javitt, D. (2012). Twenty-five years of glutamate in schizophrenia: are we there yet? Schizophr. Bull. 38, 911-913. doi: 10.1093/schbul/sbs100

Javitt, D., Zukin, S., Heresco-Lovy, U., and Umbricht, D. (2012). Has an angel shown the way? Etiological and therapeutic implications of the PCP/NMDA model of schizophrenia. Schizophr. Bull. 38, 958-966. doi: $10.1093 /$ schbul/sbs069

Jensen, O., and Mazaheri, A. (2010). Shaping functional architecture by oscillatory alpha activity: gating by inhibition. Front. Hum. Neurosci. 4:186. doi: $10.3389 /$ fnhum.2010.00186

Jobert, M., Wilson, F. J., Ruigt, G. S., Brunovsky, M., Prichep, L. S., Drinkenburg, W. H., et al. (2012). Guidelines for the recording and evaluation of pharmaco-EEG data in man: the International Pharmaco-EEG Society (IPEG). Neuropsychobiology 66, 201-220. doi: 10.1159/000343478

Jonmohamadi, Y., Poudel, G., Innes, C., and Jones, R. (2014). Source-space ICA for EEG source separation, localization and time-course reconstruction. Neuroimage 101, 720-737. doi: 10.1016/j.neuroimage.2014.07.052

Kam, J., Bolbecker, A., O'Donnell, B., Hetrick, W., and Brenner, C. (2013). Resting state EEG power and coherence abnormalities in bipolar disorder and schizophrenia. J. Psychiatr. Res. 47, 1893-1901. doi: 10.1016/j.jpsychires.2013.09.009

Kang, S., Im, C., Shim, M., Nahab, F., Park, J., Kim, D., et al. (2015). Brain networks responsible for sense of agency: an EEG study. PLoS ONE 10:e0135261. doi: 10.1371/journal.pone.0135261

Kapur, S. (2003). Psychosis as a state of aberrant salience: a framework linking biology, phenomenology, and pharmacology in schizophrenia. Am. J. Psychiatry 160, 13-23. doi: 10.1176/appi.ajp.160.1.13

Kayser, J., and Teake, C. (2010). In search of the Rosetta Stone for scalp EEG: converging on reference-free techniques. Clin. Neurophysiol. 121, 1973-1975. doi: 10.1016/j.clinph.2010.04.030

Kim, J. W., Lee, Y. S., Han, D. H., Min, K. J., Lee, J., and Lee, K. (2015). Diagnostic utility of quantitative EEG in un-medicated schizophrenia. Neurosci. Lett. 589, 126-131. doi: 10.1016/j.neulet.2014.12.064

Kittelberger, K., Hur, E., Sazegar, S., Keshavan, V., and Kocsis, B. (2012). Comparison of the effects of acute and chronic administration of ketamine in hippocampal oscillations. Relevance for the NMDA receptor hypofunction model of schizophrenia. Brain Struct. Funct. 217, 395-409. doi: 10.1007/s00429011-0351-8

Klimesch, W. (2012). Alpha-band oscillations, attention, and controlled access to stored information. Trends Cogn. Sci. 16, 606-617. doi: 10.1016/j.tics.2012.10.007

Knott, V. (2000). Quantitative EEG methods and measures in human psychopharmacological research. Hum. Psychopharmacol. 15, 479-498. doi: 10.1002/1099-1077(200010)15:7<479::AID-HUP206>3.0.CO;2-5

Knott, V., Labelle, A., Jones, B., and Mahoney, C. (2001). Quantitative EEG in schizophrenia and in response to acute and chronic clozapine treatment. Schizophr. Res. 50, 41-53. doi: 10.1016/S0920-9964(00)00165-1

Knott, V., McIntosh, J., Millar, A., Fisher, D., Villeneuve, C., Ilivitsky, V., et al. (2006). Nicotine and smoker status moderate brain electric and mood activation induced by ketamine, an N-methyl-D-aspartate (n.d.) receptor antagonist. Pharmacol. Biochem. Behav. 85, 228-242. doi: 10.1016/j.pbb.2006.08.005

Knyazeva, M., Jalili, M., Meuli, R., Hasler, M., De Feo, O., and Do, K. (2008). Alpha rhythm and hypofrontality in schizophrenia. Acta Psychiatr. Scand. 118, 188-199. doi: $10.1111 /$ j.1600-0447.2008.01227.x 
Kochs, E., Scharein, E., Möllenberg, O., Bromm, B., and Schulte am Esch, J. (1996). Analgesic efficacy of low-dose ketamine. Anaesthesiology 85, 304-314. doi: 10.1097/00000542-199608000-00012

Kocsis, B., Brown, R., McCarley, R., and Hajos, M. (2013). Impact of ketamine on neuronal network dynamics: translational modeling of schizophrenia-relevant deficits. CNS Neurosci. Ther. 19, 437-447. doi: 10.1111/cns.12081

Krystal, J., D'Souza, C., Karper, L., Bennett, A., Abi-Dargham, A., AbiSaab, D., et al. (1999). Interactive effects of subanesthetic ketamine and haloperidol in healthy humans. Psychopharmacology (Berl.) 145, 193-204. doi: 10.1007/s002130051049

Krystal, J., Karper, L., Seibyl, J., Freeman, G., Delaney, R., Bremner, J., et al. (1994). Subanaesthetic effects of the noncompetitive NMDA antagonist, ketamine, in humans. Psychotomimetic, perceptual, cognitive and neuroendocrine responses. Arch. Gen. Psychiatry 51, 199-214. doi: 10.1001/archpsyc.1994.03950030035004

Lahti, A., Holcomb, H., Medoff, D., and Tamminga, C. (1995). Ketamine activates psychosis and alters limbic blood flow in schizophrenia. Neuroreport 6, 869872. doi: 10.1097/00001756-199504190-00011

Lee, K.-H., Williams, L., Breakspear, M., and Gordon, E. (2003). Synchronous gamma activity: a review and contribution to an integrative neuroscience model of schizophrenia. Brain Res. Brain Res. Rev. 41, 57-78. doi: 10.1016/S01650173(02)00220-5

Lewis, D., Curley, A., Glausier, J., and Volk, D. (2012). Cortical paravalbumin interneurons and cognitive dysfunction in schizophrenia. Trends Neurosci. 35, 57-67. doi: 10.1016/j.tins.2011.10.004

Lovett-Barron, M., Turi, G., Kaifogh, P., Lee, P., Bolze, F., Sun, X., et al. (2012). Regulation of neuronal input transformations by tunable dendritic inhibition. Nat. Neurosci. 15, 423-430. doi: 10.1038/nn.3024

Maccaferri, G., and Dingledine, R. (2002). Control of feedforward dendritic inhibition by NMDA receptor-dependent spike timing in hippocampal interneurons. J. Neurosci. 22, 5462-5472.

Maggini, C., Raballo, A., and Salvatore, P. (2002). Depersonalization and basic symptoms in schizophrenia. Psychopathology 35, 17-24. doi: $10.1159 / 000056211$

Malhotra, A., Pinals, D., Weingortner, H., Sirocco, K., Missar, C., Pickar, D., et al. (1996). NMDA receptor function and human cognition: the effects of ketamine in healthy volunteers. Neuropsychopharmocology 14, 301-307. doi: 10.1016/0893-133X(95)00137-3

Mantini, D., Perrucci, M., Del Grotta, C., Romani, G., and Corbetta, M. (2007). Electrophysiological signatures of resting state networks in the human brain. Proc. Natl. Acad. Sci. U.S.A. 104, 13170-13175. doi: 10.1073/pnas.0700 668104

Maxwell, M. (1992). Family Interview for Genetic Studies (FIGS): Manual for FIGS. Clinical Neurogenetics Branch, Intramural Research Program. Bethesda, MD: National Institute of Mental Health.

McMenamin, B., Shackman, A., Maxwell, J., Bachhuber, D., Koppenhaver, A., Greischar, L., et al. (2010). Validation of ICA-based myogenic artifact suppression for scalp and source-localized EEG. Neuroimage 49, 2416-2432. doi: 10.1016/j.neuroimage.2009.10.010

Menon, V. (2011). Large-scale brain networks and psychopathology: a unifying triple network model. Trends Cogn. Sci. 15, 483-506. doi: 10.1016/j.tics.2011.08.003

Merlo, M., Kleinlogel, H., and Koukkou, M. (1998). Differences in the EEG profiles of early and late responders to antipsychotic treatment in first episode, drug-naive psychotic patients. Schizophr. Res. 30, 221-228. doi: 10.1016/S09209964(97)00156-4

Merritt, R., McGuire, P., and Egerton, A. (2013). Relationship between glutamate dysfunction and symptoms and cognitive function in psychosis. Front. Psychiatry 4:151. doi: 10.3389/fpsyt.2013.00151

Mima, T., and Hallett, M. (1999). Electroencephalographic analysis of cortico-muscular coherence: reference effects, volume conduction and generator mechanism. Clin. Neurophysiol. 110, 60-66. doi: 10.1016/S1388-2457(99)00238-2

Moghaddam, B., Adams, B., Verma, A., and Daly, D. (1997). Activation of glutamatergic neurotransmission by ketamine: a novel step in the pathway from NMDA receptor blockade to dopaminergic and cognitive disruptions associated with the prefrontal cortex. J. Neurosci. 17, 2921-2927.
Moran, L., and Hong, L. (2011). High vs low frequency neural oscillations in schizophrenia. Schizophr. Bull. 37, 659-663. doi: 10.1093/schbul/sbr056

Moran, R., Jones, M., Blockeel, A., Adams, R., Stephan, K., and Friston, K. (2015). Losing control under ketamine: suppressed cortico-hippocampal drive following acute ketamine in rats. Neuropsychopharmacology 40, 268-277. doi: 10.1038/npp.2014.184

Morgan, C., Perry, E., Cho, H.-S., Krystal, J., and D’Souza, D. (2006). Greater vulnerability to the amnestic effects of ketamine in males. Psychopharmacology (Berl.) 187, 405-414. doi: 10.1007/s00213-006-0409-0

Mulders, P., van Eijndhoven, P., Schene, A., Beckmann, C., and Tendolkar, I. (2015). Resting-state functional connectivity in major depressive disorder: a review. Neurosci. Biobehav. Rev. 56, 730-744. doi: 10.1016/j.neubiorev.2015.07.014

Muthukumaraswamy, S., Shaw, A., Jackson, L., Hall, J., Moran, R., and Saxena, N. (2015). Evidence that subanesthetic doses of ketamine cause sustained disruptions of NMDA and AMPA-medicated frontoparietal connectivity in humans. J. Neurosci. 35, 11694-11706. doi: 10.1523/JNEUROSCI.0903-15.2015

Narayanan, B., O’Neil, K., Berwise, C., Stevens, M., Calhoun, V., Clementz, B., et al. (2014). Resting state electroencephalogram oscillatory abnormalities in schizophrenia and psychotic bipolar patients and their relatives from the bipolar and schizophrenia network on intermediate phenotypes study. Biol. Psychiatry 76, 456-465. doi: 10.1016/j.biopsych.2013.12.008

Nekovarova, T., Fajenerova, I., Horacek, J., and Spaniel, F. (2014). Bridging disparate symptoms of schizophrenia: a triple network dysfunction theory. Front. Behav. Neurosci. 8:171. doi: 10.3389/fnbeh.2014.00171

Nicholson, C. (1973). Theoretical analysis of field potentials in anisotropic ensembles of neuronal elements. IEEE Trans. Biomed. Eng. 20, 278-288. doi: 10.1109/TBME.1973.324192

Northoff, G. (2015). Is schizophrenia a spatiotemporal disorder of the brain's resting state? World Psychiatry 14, 34-35. doi: 10.1002/wps.20177

Nottage, J. F., and Horder, J. (2015). State-of-the-art analysis of high-frequency (gamma range) electroencephalography in humans. Neuropsychobiology 72 , 219-228. doi: $10.1159 / 000382023$

Nunez, P., and Srinivasan, R. (2006). Electric Fields of the Brain: The Neurophysics of EEG, 2nd Edn. New York, NY: Oxford University Press.

Nunez, P., Srinivasan, R., Westdorp, A., Wijesinghe, R., Tucker, D., Silberstein, R., et al. (1997). EEG coherency. I: statistics, reference electrode, volume conduction, Laplacians, cortical imaging, and interpretation at multiple scales. Electroencephalogr. Clin. Neurophysiol. 103, 499-515. doi: 10.1016/S00134694(97)00066-7

Olney, J., and Farber, N. (1995). Glutamate receptor dysfunction and schizophrenia. Arch. Gen. Psychiatry 52, 998-1007. doi: 10.1001/archpsyc. 1995.03950240016004

Palaniyappan, L., and Liddle, P. (2012). Does the salience network play a cardinal role in psychosis? An emerging hypothesis of insular dysfunction. J. Psychiatry Neurosci. 37, 17-27. doi: 10.1503/jpn.100176

Palaniyappan, L., White, T., and Liddle, P. (2012). The concept of salience network dysfunction in schizophrenia: from neuroimaging observations to therapeutic outcomes. Curr. Top. Med. Chem. 12, 2324-2338. doi: $10.2174 / 156802612805289881$

Pascual-Marqui, R. (2011). Discrete, 3D Distributed, Linear Imaging Methods of Electric Neuronal Activity. Part I: Exact, Zero Error Localization. arXiv:0710.3341 [math-ph]. Available at: http://arxiv.org/pdf/0710.3341

Pascual-Marqui, R., Lehman, D., Koukkou, M., Kochi, K., Anderer, P., Saletu, B., et al. (2011). Assessing interactions in the brain with exact low resolution electromagnetic tomography (eLORETA). Philos. Trans. A Math. Phys. Eng. Sci. 360, 3768-3784. doi: 10.1098/rsta.2011.0081

Perrin, F., Fernier, J., Bertrand, O., and Echallier, J. (1989). Spherical spline for potential and current density napping. Electroencephalogr. Clin. Neurophysiol. 72, 184-187. doi: 10.1016/0013-4694(89)90180-6

Pinault, D. (2008). N-methyl-D-aspartate receptor antagonists ketamine and MK801 induce wake-related aberrant gamma oscillations in the rat neocortex. Biol. Psychiatry 63, 730-735. doi: 10.1016/j.biopsych.2007.10.006

Poels, E., Kegeles, L., Kantrowitz, J., Slifstein, M., Javitt, D., Lieberman, J., et al. (2014). Imaging glutamate in schizophrenia: review of findings and implications for drug discovery. Mol. Psychiatry 19, 20-29. doi: $10.1038 / \mathrm{mp} .2013 .136$ 
Qin, P., and Northoff, G. (2011). How is our self related to midline regions and the default-mode network? Neuroimage 57, 1221-1233. doi: 10.1016/j.neuroimage.2011.05.028

Raballo, A. (2012). Self-disorders and the experimental core of schizophrenia spectrum vulnerability. Psychiatr. Danub. Suppl. 3, S303-S310.

Raichle, M., MacLeod, A., Synder, A., Powers, W., Gusnard, D., and Shulman, G. (2001). A default mode of brain function. Proc. Natl. Acad. Sci. U.S.A. 98, 678-682. doi: 10.1073/pnas.98.2.676

Ranlund, S., Nottage, J., Shaikh, M., Dutt, A., Constante, M., Walshe, M., et al. (2014). Resting EEG in psychosis and at-risk populations - a possible endophenotype? Schizophr. Res. 153, 96-102. doi: 10.1016/j.schres.2013.12.017

Rivolta, D., Heidegger, T., Scheller, B., Sauer, A., Schaum, M., Birkner, K., et al. (2015). Ketamine dysregulates the amplitude and connectivity of highfrequency oscillations in cortical-subcortical networks in humans: evidence from resting-state magnetoencephalography-recordings. Schizophr. Bull. 4, 1105-1114. doi: 10.1093/schbul/sbv051

Roopun, A., Cunningham, M., Racca, C., Alter, K., Traub, R., and Whittington, M. (2008). Region-specific changes in gamma and beta2 rhythms in NMDA receptor dysfunction models of schizophrenia. Schizophr. Bull. 34, 962-973. doi: $10.1093 / \mathrm{schbul} / \mathrm{sbn} 059$

Royer, S., Zemelman, B., Losonczy, A., Kim, J., Chance, F., Magee, J., et al. (2012). Control of timing, rate and bursts of hippocampal place cells by dendritic and somatic inhibition. Nat. Neurosci. 15, 769-775. doi: 10.1038/nn.3077

Rutter, L., Carver, F., Holroyd, T., Nadar, S., Mitchell-Francis, J., Apud, J., et al. (2009). Mangetoencephalographic gamma power reduction in patients with schizophrenia during resting condition. Hum. Brain Mapp. 30, 3254-3264. doi: $10.1002 / \mathrm{hbm} .20746$

Saletu, B., Anderer, P., and Saletu-Zyblarz, G. (2006). EEG topography and tomography (LORETA) in the classification and evaluation of the pharmacodynamics of psychotropic drugs. Clin. EEG Neurosci. 37, 66-80. doi: $10.1177 / 155005940603700205$

Sanacora, G., Smith, M., Pathak, S., Su, H., Boeijinga, P., McCarthy, D., et al. (2014). Lanicemine: a low-trapping NMDA channel blocker produces sustained antidepressant efficacy with minimal psychotomimetic adverse effects. Mol. Psychiatry 19, 978-985. doi: 10.1038/mp.2013.130

Saunders, J., Gandal, M., and Siegel, S. (2012). NMDA antagonists recreate signal to-noise ration and timing perturbations present in schizophrenia. Neurobiol. Dis. 46, 93-100. doi: 10.1016/j.nbd.2011.12.049

Scherbinin, S., Doyle, O., Zelaya, F., de Simoni, S., Mehta, M., and Schwarz, A. (2015). Modulatory effects of ketamine, risperidone and lamotrigine on resting brain perfusion in healthy human subjects. Psychopharmacology (Berl.) 232, 4191-4204. doi: 10.1007/s00213-015-4021-Z

Schiavone, S., Jaquet, V., Sorce, S., Dubois-Dauphin, M., Hultqvist, M., Bäckdahl, L., et al. (2012). NADPH oxidase elevations in pyramidal neurons drive psychosocial stress-induced neuropathology. Transl. Psychiatry 2:e111. doi: $10.1038 /$ tp. 2012.36

Schiavone, S., Mhillaj, E., Neri, M., Morgese, M. G., Tucci, P., Bove, M., et al. (2016). Early loss of blood-brain barrier integrity precedes NOX2 elevation in the prefrontal cortex of an animal model of psychosis. Mol. Neurobiol. doi: 10.1007/s12035-016-9791-8 [Epub ahead of print].

Schultz, A., Schultz, B., Zachen, B., and Pichlmayr, I. (1990). The effects of ketamine on the electroencephalogram - typical patterns and spectral representations. Anaesthetist 39, 222-225.

Smith, S., Fox, P., Miller, K., Glahn, D., Fox, P., Mackay, C., et al. (2009). Correspondence of the brain; functional architecture during activation and rest. Proc. Natl. Acad. Sci. U.S.A. 106, 13040-13045. doi: 10.1073/pnas.0905267106

Sorce, S., and Krause, K. H. (2009). NOX enzymes in the central nervous system: from signaling to disease. Antioxid. Redox Signal. 11, 2481-2504. doi: 10.1089/ARS.2009.2578

Sorce, S., Schiavone, S., Tucci, P., Colaianna, M., Jaquet, V., Cuomo, V., et al. (2010). The NADPH oxidase NOX2 controls glutamate release: a novel mechanism involved in psychosis-like ketamine responses. J. Neurosci. 30, 11317-11325. doi: 10.1523/JNEUROSCI.1491-10.2010

Spencer, K. (2014). Time to be spontaneous: a renaissance of intrinsic brain activity in psychosis research? Biol. Psychiatry 76, 431-435. doi: 10.1016/j.biopsych.2014.07.009
Sponheim, S., Clementz, B., Iacono, W., and Beiser, M. (1994). Resting EEG in first-episode and chronic schizophrenia. Psychophysiology 31, 37-43. doi: 10.1111/j.1469-8986.1994.tb01023.x

Sponheim, S., Clementz, B., Iacono, W., and Beiser, M. (2000). Clinical and biological concomitants of resting state EEG lower abnormalities in schizophrenia. Biol. Psychiatry 48, 1088-1097. doi: 10.1016/S00063223(00)00907-0

Sridharan, D., Levitin, D., and Menon, V. (2008). A cortical role for the right fronto-insular cortex in switching between central-executive and defaultmode networks. Proc. Natl. Acad. Sci. U.S.A. 105, 12569-12574. doi: $10.1073 /$ pnas. 0800005105

Stahl, S. (2013). Mechanisms of action of ketamine. CNS Spectr. 18, 171-174. doi: 10.1017/S109285291300045X

Sun, Y., Farzan, F., Barr, M., Kirihara, K., Fitzgerald, P., Light, G., et al. (2011) Gamma oscillations in schizophrenia: mechanisms and clinical significance. Brain Res. 1413, 98-114. doi: 10.1016/j.brainres.2011.06.065

Tikka, S., Nizamie, S., Das, B., Katshu, M., and Goyal, N. (2013). Increased spontaneous gamma power and synchrony in schizophrenia patients having higher minor physical anomalies. Psychiatry Res. 107, 164-172. doi: 10.1016/j.psychres.2012.09.006

Tikka, S., Nizamie, S., Goyal, N., Pradhan, N., Tikka, D., and Katshu, M (2015). Evaluation of spontaneous dense array gamma oscillatory activity and minor physical anomalies as a composite neurodevelopmental endophenotype in schizophrenia. Int. J. Dev. Neurosci. 40, 43-51. doi: 10.1016/j.ijdevneu.2014.11.002

Tikka, S., Yadav, S., Nizamie, S., Das, B., Goyal, N., and Tikka, D. (2014). Sporadic and familial subgroups of schizophrenia do not differ on dense array spontaneous gamma oscillatory activity. Psychiatry Res. 220, 1131-1154. doi: 10.1016/j.psychres.2014.08.042

Uhlhaas, P., and Singer, W. (2010). Abnormal neural oscillations and synchrony in schizophrenia. Nat. Rev. Neurosci. 11, 100-113. doi: 10.1038/nrn2774

Venables, N., Bernat, E., and Sponheim, S. (2009). Genetic and disorder-specific aspects of resting state EEG abnormalities in schizophrenia. Schizophr. Bull. 35, 826-839. doi: 10.1093/schbul/sbn021

Vincent, J., Patel, G., Fox, M., Snyder, A., Baker, J., Van Essen, D., et al. (2007) Intrinsic functional architecture in the anaesthetized monkey brain. Nature 447 , 83-86. doi: $10.1038 /$ nature 05758

Vollenweider, F., Leenders, K., Scharfetter, C., Antonini, A., Maguire, P., Missimer, J., et al. (1997). Metabolic hyperfrontality and psychopathology in the ketamine model of psychosis using positron emission tomography (PET) and [18F] fluorodeoxyglucose (FDG). Eur. Neuropsychopharmacol. 7, 9-24. doi: 10.1016/S0924-977X(96)00039-9

Wada, Y., Takizawa, Y., Kitazawa, S., Jiang, Z., and Yamaguchi, N. (1994). Quantitative EEG analysis at rest and during photic stimulation in drug-naïve patients with first-episode. Eur. Arch. Psychiatry Clin. Neurosci. 244, 247-251. doi: 10.1007/BF02190377

Williams, J., Gibbon, M., First, M., Spitzer, R., Davies, M., Borus, J., et al. (1992). The Structural Clinical Interview for the DSM-III-R (SCID). Multisite test-retest reliability. Arch. Gen. Psychiatry 49, 630-636. doi: 10.1001/archpsyc.1992.01820080038006

Yuan, H., Zotev, V., Phillips, R., Drevets, W., and Bodurka, J. (2012) Spatiotemporal dynamics of the brain at rest - Exploring EEG microstates as electrophysiological signatures of BOLD resting state networks. Neuroimage 60 , 2062-2072. doi: 10.1016/j.neuroimage.2012.02.031

Conflict of Interest Statement: The authors declare that the research was conducted in the absence of any commercial or financial relationships that could be construed as a potential conflict of interest.

Copyright (c) 2016 de la Salle, Choueiry, Shah, Bowers, McIntosh, Ilivitsky and Knott. This is an open-access article distributed under the terms of the Creative Commons Attribution License (CC BY). The use, distribution or reproduction in other forums is permitted, provided the original author $(s)$ or licensor are credited and that the original publication in this journal is cited, in accordance with accepted academic practice. No use, distribution or reproduction is permitted which does not comply with these terms. 
Appendix II: NMDA Receptor Antagonist Effects on Speech-Related Mismatch Negativity and Its Underlying Oscillatory and Source Activity in Healthy Humans (Published in Frontiers in Pharmacology:

Neuropharmacology)

\section{OPEN ACCESS}

Edited by: Adrian Preda, University of California, Irvine, United States

Reviewed by: Kenji Hashimoto, Chiba University, Japan Anna Maria Pittaluga, University of Genoa, Italy Holly Hamilton,

University of California, San Francisco, United States

${ }^{*}$ Correspondence: Verner Knott verner.knott@theroyal.ca

Specialty section: This article was submitted to

Neuropharmacology,

a section of the journal Frontiers in Pharmacology

Received: 18 September 2018 Accepted: 11 April 2019 Published: 08 May 2019

Citation: de la Salle S, Shah D, Choueiry J, Bowers H, Mclntosh J, llivitsky V and Knott V (2019) NMDA Receptor Antagonist Effects on Speech-Related Mismatch Negativity and Its Underlying Oscillatory and Source Activity in Healthy Humans.

Front. Pharmacol. 10:455. doi: 10.3389/fphar.2019.00455

\section{NMDA Receptor Antagonist Effects on Speech-Related Mismatch Negativity and Its Underlying Oscillatory and Source Activity in Healthy Humans}

\author{
Sara de la Salle1, Dhrasti Shah ${ }^{1}$, Joelle Choueiry², Hayley Bowers ${ }^{3}$, Judy Mclntosh ${ }^{4}$, \\ Vadim Ilivitsky ${ }^{5}$ and Verner Knott ${ }^{1,2,4,5 *}$ \\ 'School of Psychology, University of Ottawa, Ottawa, ON, Canada, ${ }^{2}$ Department of Cellular and Molecular Medicine, \\ University of Ottawa, Ottawa, ON, Canada, ${ }^{3}$ Department of Psychology, University of Guelph, Guelph, ON, Canada, \\ ${ }^{4}$ The Royal's Institute of Mental Health Research, Ottawa, ON, Canada, ${ }^{5}$ Royal Ottawa Mental Health Centre, Ottawa, \\ ON, Canada
}

Background: Previous studies in schizophrenia have consistently shown that deficits in the generation of the auditory mismatch negativity (MMN) - a pre-attentive, eventrelated potential (ERP) typically elicited by changes to simple sound features - are linked to $\mathrm{N}$-methyl-D-aspartate (NMDA) receptor hypofunction. Concomitant with extensive language dysfunction in schizophrenia, patients also exhibit MMN deficits to changes in speech but their relationship to NMDA-mediated neurotransmission is not clear. Accordingly, our study aimed to investigate speech MMNs in healthy humans and their underlying electrophysiological mechanisms in response to NMDA antagonist treatment. We also evaluated the relationship between baseline MMN/electrocortical activity and emergent schizophrenia-like symptoms associated with NMDA receptor blockade.

Methods: In a sample of 18 healthy volunteers, a multi-feature Finnish language paradigm incorporating changes in syllables, vowels and consonant stimuli was used to assess the acute effects of the NMDA receptor antagonist ketamine and placebo on the MMN. Further, measures of underlying neural activity, including evoked theta power, theta phase locking and source-localized current density in cortical regions of interest were assessed. Subjective symptoms were assessed with the Clinician Administered Dissociative States Scale (CADSS).

Results: Participants exhibited significant ketamine-induced increases in psychosislike symptoms and depending on temporal or frontal recording region, co-occurred with reductions in MMN generation in response to syllable frequency/intensity, vowel duration, across vowel and consonant deviants. MMN attenuation was associated with decreases in evoked theta power, theta phase locking and diminished current density in auditory and inferior frontal (language-related cortical) regions. Baseline (placebo) MMN and underlying electrophysiological features associated with the processing of changes in syllable intensity correlated with the degree of psychotomimetic response to ketamine. 
Conclusion: Ketamine-induced impairments in healthy human speech MMNs and their underlying electrocortical mechanisms closely resemble those observed in schizophrenia and support a model of dysfunctional NMDA receptor-mediated neurotransmission of language processing deficits in schizophrenia.

\section{HIGHLIGHTS}

- Neural effects of NMDA receptor blockade on speech processing were assessed in a ketamine model.

- Ketamine reduced MMN, theta power, theta phase locking factor and regional cortical current density.

- Psychosis-like symptoms induced by ketamine were related to baseline (placebo) neural measures of speech processing.

Keywords: mismatch negativity, $N$-methyl-D-aspartate (NMDA), ketamine, theta oscillations, cortical current density

\section{INTRODUCTION}

This electroencephalographic (EEG)-based research project aimed to advance our neural understanding of language processing by assessing the effects of ketamine-induced $\mathrm{N}$-methyl-D-aspartate (NMDA) receptor hypofunction on mismatch negativity (MMN) responses to changes in speech stimuli (i.e., speech deviants). We also investigated stimuluslocked time-frequency and event-related spectral perturbation (ERSP) signatures of ketamine-induced changes in speech MMNs. Although frequently assessed in relation to high frequency (beta and gamma) oscillations (Lee et al., 2018), we specifically focused on the evoked and phase-locking activity of the theta band as viable translational biomarkers for investigating brain dynamics underlying language processing deficits, and their treatment with NMDA receptor antagonists. To examine ketamine effects on speech processing at the oscillatory and network level, we combined ERP and ERSP approaches with a source localization technique, using lowresolution brain electromagnetic tomography (LORETA) to assess $\mathrm{MMN}$-associated current density in auditory and language related regions of interest. Addressing the clinical relevance of this work, as multiple lines of evidence have implicated NMDA receptor dysfunction in schizophrenia pathophysiology including impairments in MMN generation, this study examined the relationship of these neural measures to psychotomimetic symptoms produced by acute ketamine administration. This is the first known human EEG study combining sensor- and sourcelevel approaches to assess the neural substrates of speech deviance detection modified by NMDA receptor antagonist treatment and their association with schizophrenia-like symptomatology.

\section{BACKGROUND}

\section{Event-Related Potentials (ERP)}

Neurophysiological approaches such as EEG-derived averaged event-related potentials (ERP) are used increasingly as biomarkers for aiding our understanding the neural mechanisms underlying normal and abnormal information processing, and to monitor novel drug therapies for treatment of sensory and cognitive processing deficits (Javitt et al., 2008; Bickel and Javitt, 2009; Javitt, 2015). Within the auditory system, sensory processing is reflected in the generation of the MMN, an ERP reflecting the function of the auditory "echoic" memory system, which maintains brief representations of auditory stimulus features. This automatic change-detection response is elicited when the brain detects an infrequent deviance in a stream of sound stimulation, even in the absence of attention (Näätänen and Alho, 1997; Näätänen et al., 2007; Paavilainen, 2013). The MMN is most commonly recorded in the context of the basic "oddball paradigm" where a physically constant "standard" tone is infrequently replaced by a "deviant" tone (e.g., differing in frequency, duration or intensity). The MMN elicited by the deviance is best observed in the deviant-minus-standard ERP as a frontocentrally distributed negativity, typically peaking at 150-200 ms after the onset of deviant stimulus. As MMN and its abnormality can also be elicited by changes of an abstract nature such as a violation of a multi-stimulus pattern or regularity (Avissar et al., 2018; Salisbury et al., 2018), or that of a complex sequential stimulus rule, the $\mathrm{MMN}$ is thought to signal a prediction-error based on regularity-violation rather than just stimulus-change (Todd et al., 2013).

\section{N-Methyl-D-Aspartate (NMDA) Receptor Function}

$\mathrm{N}$-methyl-D-aspartate receptors are widely expressed in sensory and higher cognitive brain regions, and dose-dependent MMN deficits are observed with either systemic or local infusion of NMDA receptor antagonists directly into the auditory cortex (Javitt et al., 1996; Javitt, 2000). Evidence in animal models of the role of NMDA receptor hypofunction, either genetic (Featherstone et al., 2015) or pharmacologically induced, on aberrant MMN generation (Harms, 2016) is paralleled by studies in healthy human volunteers administered 
acute sub-anesthetic doses of ketamine. Ketamine is a noncompetitive NMDA receptor antagonist which reduces and delays MMN generation elicited by frequency and duration tone deviants (Rosburg and Kreitschmann-Andermahr, 2016). MMN generation is not modulated either by dopamine (D1/D2) agonists or by psychotomimetics targeting serotonin (5-HT2H) receptors (Bickel and Javitt, 2009; Kantrowitz and Javitt, 2010). Suggesting both limited dopaminergic involvement and relative specificity of NMDA receptor antagonist effects, MMN is considered to be a simple and usefu biomarker of NMDA receptor-type glutamate dysfunction (Avissar and Javitt, 2018).

Mismatch negativity has been increasingly used for studying hierarchical levels of acoustic processing involved in speech and language function. General findings of larger MMNs to speech sounds and words compared with unfamiliar sounds and pseudo-words, respectively, suggests that MMN can serve as a reliable probe of phonological, lexical, semantic, and syntactic processes (Näätänen, 1999; Pulvermuller and Shtyrov, 2006; Shtyrov and Pulvermuller, 2007). Our primary objective was to assess the effects of ketamine-induced NMDA receptor hypofunction on speech MMNs, assessing MMN response to ketamine as a function of 5 speech deviants, including changes in syllable frequency, syllable intensity, vowel duration, as well as consonant change and across-vowel changes. Accurate perception of acoustic features of spoken words (e.g., frequency and intensity) and their temporal attributes (e.g., duration or determining where a work, phrase, and sentence ends) are essential for affective social communication and there is evidence that neural processing of phonological features differs from that of non-linguistic auditory information (Näätänen, 1999; Pulvermuller and Shtyrov, 2006; Shtyrov and Pulvermuller, 2007). As meta-analyses of studies examining MMN response to ketamine have shown equivalent reductions in duration and frequency MMNs, we also expect that ketamine will impair MMN generation across deviant types.

\section{ERO Time-Frequency Analysis}

A mechanistic understanding of the effects of NMDA receptor function on $\mathrm{MMN}$ requires an evaluation of its impact on event-related spectral oscillatory (ERO) activity across different frequency bands [delta $(<4 \mathrm{~Hz})$, theta $(4-8 \mathrm{~Hz})$, alpha $(8-12 \mathrm{~Hz})$, beta $(12-30 \mathrm{~Hz})$, gamma $(30-80 \mathrm{~Hz})]$. EROs can be obtained at levels ranging from single cell to focal field potentials in animals, to large-scale synchronized activities measured at the human scalp (Moran and Hong, 2011). Power in the theta band is particularly prominent during the processing of tone and speech deviants, with studies showing that neural generation of the MMN is accompanied by theta power modulation and theta phase alignment (Fuentemilla et al., 2008; Hsiao et al., 2009; Bishop and Hardiman, 2010; Ko et al., 2012; Choi et al., 2013; Hermann et al., 2014; Koerrner et al., 2016; Corcoran et al., 2018). NMDA receptor antagonists modulate background spontaneous theta oscillations (Lazarewicz et al., 2009; Hunt and Kasicki, 2013) and reduce both MMN and the theta response to auditory deviants in rodents (Lee et al., 2018). Thus, evoked theta activity is proposed as a viable translational biomarker for investigating brain dynamics underlying auditory processing deficits and their treatment with NMDA receptor antagonists. In addition to measuring the power of oscillations associated with the averaged time-domain ERPs, sensitive 'time-frequency' analyses of trial-by-trial ERSPs are being increasingly used to provide a more detailed picture of brain dynamics underlying ERPs, which is not available with the averaged time-domain ERP, as seen with the MMN waveform (Lee et al., 2017). One of the most frequently used ERSP measures, which is computed on a trial-by-trial basis and is known to independently (vs. power) affect the amplitude of the averaged ERP, is the phase locking factor [PLF; also known as phase locking index (PLI), or intertrial phase coherence (ITC)]. This measures the degree of phase consistency across trials, and is commonly interpreted to reflect phase resetting of ongoing oscillations. The frontal MMN has been found to be associated with an increase in theta power for deviant stimuli as well as by theta phase alignment, whereas the temporal MMN has been found to be associated with theta phase resetting with no power modulation (Fuentemilla et al., 2008). This study investigated the time-frequency signature of ketamine-induced changes in speech MMNs with a specific focus on evoked theta power and theta phase locking.

\section{ERP Source Activity}

The neural characteristics of the MMN (amplitude, power, and phase resetting) are presumed to result from activity of neural networks involved in its generation (Fuentemilla et al., 2008). Studies employing dynamic causal modeling, source localization, functional magnetic resonance imaging (fMRI) and positron emission tomography (PET) have shown the MMN to be generated by neural activity and connectivity within a hierarchically organized cortical network involving temporal (bilateral auditory cortex) and predominantly right frontal brain regions (Näätänen and Alho, 1997; Näätänen et al., 2007). In addition to primary ( $\mathrm{pAC}$ ) and secondary ( $\mathrm{sAC}$ ) auditory cortices in the temporal lobe, the inferior frontal gyrus (IFG) is the most studied non-auditory cortical contributor to MMN generation and appears to serve as an evaluator of stimulus relevance (Todd et al., 2012). In the oddball paradigm, its activity may reflect the importance of the violated expectation (prediction error) detected by auditory cortical areas (Todd et al., 2012). Activity within and between cortical areas is essential for auditory perception and oscillatory activity plays a cardinal role in the recruitment of brain regions and their coupling (Lee et al., 2017). Neurons within the IFG, such as those located in Broca's language area and Broca's right homolog, are characterized by a well-described theta frequency synchronization with the auditory cortex (Hsiao et al., 2009; Choi et al., 2013). In order to examine ketamine effects on speech processing at the sensory and network levels, we combined ERP and time-frequency/ERSP approaches with a source localization technique, using low-resolution brain electromagnetic tomography (LORETA), a 3D inverse solution, to assess intracerebral current density as a measure of activation of regions of interest (ROI: pAC, sAC, and IFG) during MMN generation. With ERPs and their underlying spectrally analyzed activity, we expected to see ketamine-induced reductions in MMN amplitude, evoked theta power and theta phase resetting 
(phase-locking), while with source analysis, we predicted reduced current density (activation) in temporal and frontal cortical areas.

\section{Relevance for Schizophrenia}

For a number of reasons, this line of research has potential clinical implications for schizophrenia cognition (Green, 2006; Javitt, 2009a,b; Leitman et al., 2010; Javitt and Freedman, 2015; Javitt and Sweet, 2015) and particularly with respect to improving our understanding of low-level acoustic processing involved in speech and language functions that are distinctively impaired in schizophrenia (Revheim et al., 2014; Brown and Kuperburg, 2015). Among these reasons are: a vast neuroimaging, molecular, and genetic literature supporting a glutamate hypothesis of schizophrenia involving NMDA receptor hypofunction (Carlsson et al., 1999; Coyle et al., 2010; Javitt et al., 2012; Moghaddam and Javitt, 2012; Moghaddam and Krystal, 2012); pharmacological modeling of schizophrenia features, including clinical, cognitive, and sensory deficit symptoms in rodents/healthy humans with acute sub-anesthetic doses of the NMDA receptor antagonist ketamine (Pauvermann et al., 2017; Thomas et al., 2017); findings in schizophrenia of reliable and robust attenuation of the MMN (effect size $d \sim 1.00$ ), which is not affected by antipsychotics (Näätänen and Kakkonen, 2009), is more apparent with speech MMNs (Kasai et al., 2002; Fisher et al., 2008) and is associated with reductions in frontotemporal volume/function (Kasai et al., 2002; Kasai, 2004; Yamasue et al., 2004) and diminished theta responses to auditory deviants (Rodionov et al., 2009; Hong et al., 2012; Kaser et al., 2013; Kirino, 2017; Lee et al., 2017). As reduced MMNs predict conversion to psychosis in clinical high risk and reflect a vulnerability to disease progression (Näätänen et al., 2015), and as smaller MMNs to tone deviants are associated with greater vulnerability to NMDA receptor system disruption with ketamine in healthy humans (i.e., they experience greater psychosis-like symptoms; Umbricht et al., 2002) a secondary objective of this study is to examine the relationship between baseline (placebo) frontal speech MMN/oscillatory and source activity and subjective psychotomimetic response to ketamine.

\section{MATERIALS AND METHODS}

\section{Experimental Subjects}

Twenty healthy, medication-free, non-smoking, right-handed males $($ age $=20.94, S D=2.44)$ were recruited through city/university newspaper advertisements. Only males were recruited in order to avoid potential confounding effects of hormonal variations associated with the menstrual cycle in females. Only non-smokers were sampled in order to reduce any potential confounding effects of nicotine and/or nicotine withdrawal symptoms on ketamine response. Following data processing, two of the participants exhibited marked artifact contamination in their EEGs and the final study sample was limited to $N=18$. Participants were initially screened by telephone for non-smoker status (defined as not smoking a lifetime total of $>100$ cigarettes, with no smoking in the past 12 months), absence of medical illnesses, as well as psychiatric and alcohol/drug dependence disorders. They were then assessed for psychopathology and personal/family psychiatric history during an in-person screening session using the Structured Clinical Interview (SCID-non-patient version; Williams et al., 1992) for DSM-IV and the Family Interview for Genetic Studies (FIGS; Maxwell, 1992). They also underwent a full medical examination (including ECG), laboratory blood testing (to rule out any significant medical conditions), urine toxicology (to screen for psychoactive substances), an expired air carbon monoxide test to confirm non-smoker status $[<3$ parts per million (ppm)], and auditory threshold testing to ensure normal hearing.

Any participants with a current/past or family history of an axis 1-2 DSM-IV disorder, abnormal blood/ECG results, positive drug screen (for barbiturates, ketamine, cocaine, benzodiazepines, ethanol, cannabinoids, and opioids), significant medical illnesses [including seizures and recent ( $<6$ months) head trauma with loss of consciousness], or audiometric assessed indication of abnormal hearing (hearing threshold above $30 \mathrm{~dB}$ SPL for $1,000 \mathrm{~Hz}$ pure tone) were excluded. The study was limited to males to avoid possible variations related to menstrual cycle. All participants provided written informed consent during the in-person screening session and were compensated $\$ 75$ CAD per test session. This study was approved by the Research Ethics Board of the Royal Ottawa Health Care Group and was conducted in accordance with the Tri-Council Policy Statement on Ethical Conduct for Research Involving Humans.

\section{Study Design}

Participants were assessed within a randomized, double-blind design consisting of two test sessions separated by a minimum of 5 days. Half received the placebo (saline) in their first test session and ketamine in their second test session, while the other half received the treatments in the reverse order.

\section{Drug Administration}

A racemic ketamine or $\mathrm{NaCl} 0.90 \%$ w/v bolus dose was administered over $10 \mathrm{~min}(0.26 \mathrm{mg} / \mathrm{kg})$, followed immediately by a constant infusion lasting $60 \mathrm{~min}(0.65 \mathrm{mg} / \mathrm{kg})$, using an Imed Gemini PC-1 pump. The ketamine dose is consistent with previous studies (Krystal et al., 1999; Morgan et al., 2004; Lazarewicz et al., 2009; Kaser et al., 2013).

\section{Study Procedures}

Test sessions occurred in the morning (beginning at 8:00 a.m.), following overnight abstinence from caffeine, food, drinks (except for water), and alcohol. Sessions began with the insertion of an antecubital intravenous line, attachment of a 2-lead ECG (for continuous safety monitoring), and electrode positioning, followed by the administration of the bolus dose and constant infusion. After a $10 \mathrm{~min}$ stabilization period (i.e., $10 \mathrm{~min}$ into the infusion), EEG recording and auditory MMN paradigm administration began. This was followed by an evaluation with the Clinician Administered Dissociative States Scale (CADSS; Bremner et al., 1998), which is composed of 19 self-report items 
$(0=$ not at all; $1=$ slightly; $2=$ moderately; 3 = considerably; $4=$ extremely) and yields three subjective sub-scale scores (amnesia, depersonalization, and derealization) as well as 8 clinician rated items which yields an "observer" sub-scale score. Vital signs and adverse events were analyzed for safety purposes only. Participants remained in the lab until all symptoms had ceased and were not allowed to drive vehicles to or from the test sessions.

\section{MMN Paradigm}

During MMN paradigm administration, participants viewed a silent video (The Blue Planet by British Broadcasting Corporation et al., 2002) while stimuli were presented binaurally through noise-canceling headphones. A fast multi-feature speech paradigm (Pakarinen et al., 2009) was employed to acquire $\mathrm{MMNs}$ to phonetic and acoustic changes in speech stimuli. Compared to standard oddball paradigms, which allow for assessment of cortical discrimination of 1-2 sounds features, multi-feature MMN paradigms allow for very fast assessment of extensive auditory discrimination profiles, evaluating central auditory discrimination of multiple auditory attributes (Näätänen et al., 2004). The stimuli consisted of semisynthetic Finnish-language consonant-vowel (CV) syllables. The standard stimuli were /te:/ and /pi:/ (frequency $=101 \mathrm{~Hz}$, intensity $=70 \mathrm{~dB}$, syllable duration $=170 \mathrm{~ms}$ ). The deviant stimuli differed from the standards either in syllable FREQUENCY (FO $\pm 8 \%$; 93/109 Hz), syllable INTENSITY ( $\pm 6 \mathrm{~dB})$, VOWELDURATION (-70 ms; $100 \mathrm{~ms} / \mathrm{te} / \mathrm{and} / \mathrm{pi} /$ ), CONSONANT (/pe:/ and /ti:/), or VOWEL (/ti:/ and /pe:/). Additional details regarding the creation of these stimuli can be found in Pakarinen et al. (2009). The presentation of these stimuli followed the same sequence as previously outlined (Näätänen et al., 2004): every other syllable was a standard $(P=0.5)$ and every other one of the 5 deviant syllables $(P=0.1$ each). There were four 5 -min sequences including 465 syllables, of which the first 5 were always standards. In two of the sequences the standard syllable was /te:/ and the deviants were FREQUENCY /te:/, INTENSITY /te:/, VOWEL DURATION /te/, CONSONANT /pe:/, and VOWEL /ti:/, whereas in the other two sequences the standard was /pi:/, and the deviants were FREQUENCY /pi:/, INTENSITY /pi:/, VOWEL DURATION /pi/, CONSTONANT /ti:/, and VOWEL /pe:/. The occurrence of the deviants were pseudo-randomized in a way that all 5 deviants appeared once in an array of 10 successive stimuli and the same deviant was never repeated after the standard following it. The order of the two sequences was randomized between participants. Each deviant type was presented 184 times, the stimulusonset asynchrony (SOA, onset to onset) was $650 \mathrm{~ms}$, and the total recording time was $20 \mathrm{~min}$. Although use of a native (English) speech paradigm may have been preferable with our English speaking sample, this was the only published 'optimal' paradigm that allowed for quick, efficient assessment of multiple speech deviant types in a single recording when we initiated our study. The five speech deviations are generally relevant features in speech sounds across most, if not all, spoken languages. The use of a non-native paradigm to derive automatic cortical discrimination profiles has the advantage of reducing the potential confounding influence of higher-order semantic processes (on MMN generation), which are known to interact with emotional processes that are markedly impaired in schizophrenia as reflected by MMN-indexed auditory emotion recognition deficits (Kantrowitz et al., 2015).

\section{ERP Recording}

Electroencephalographic activity was recorded using current pharmaco-EEG standards (Knott, 2000; Saletu et al., 2006; Jobert et al., 2012). This involved the use of a cap (EasyCap, Herrsching-Breitbrunn, Germany) embedded with 30 $\mathrm{Ag}^{+} / \mathrm{Ag}^{+} \mathrm{Cl}^{-}$electrodes, left and right mastoids (TP9 and TP10), as well as 2 bipolar electrodes placed on the supraand sub-orbital ridge and external canthi (to measure vertical and horizontal electro-oculographic activity). An electrode on the nose served as the reference and a ground electrode was positioned at AFz. Electrical recordings were carried out using a Brain Vision QuickAmp ${ }^{\circledR}$ (Brain Products $\mathrm{GmbH}$, Munich, Germany) amplifier and Brain Vision Recorder ${ }^{\circledR}$ (Brain Products GmbH, Munich, Germany) software. Electrical activity was sampled at $500 \mathrm{~Hz}$, with amplifier bandpass filters set at 0.1-100.0 Hz. Electrode impedances were $<5 \mathrm{k} \Omega$.

\section{MMN Processing}

Mismatch negativity analysis was completed using Brain Vision ${ }^{\circledR}$ Analyzer 2 software (Brain Products, Munich, Germany). Offline pre-processing involved a visual inspection of the recordings for contamination due to noticeable ocular/muscle/cardiac activity and/or drowsiness, digital filtering at $1-20 \mathrm{~Hz}$, ocular correction with an algorithm (Gratton et al., 1983), segmentation ( -100 to $400 \mathrm{~ms})$, semi-automatic artifact rejection $( \pm 100 \mu \mathrm{V})$, and baseline correction (relative to the pre-stimulus segment). The remaining epochs (minimum 100 per deviant) were then averaged based on stimulus type, and subtraction waveforms were computed (deviant - standard) by digital point-by-point subtraction of standard waveform voltage values from each deviant waveform values. For the purposes of our study and based on grand average waveforms, frontal MMN at Fz, F3 and F4 scalp sites was quantified in terms of peak amplitude (maximum negative voltage relative to average pre-stimulus voltage within the $120-250 \mathrm{~ms}$ window for frequency, intensity, duration, vowel, deviants and the 150-280 ms window for consonant deviants). MMN latency (relative to stimulus onset) for each of the deviants, was quantified only at the midfrontal $(\mathrm{Fz})$ site. The mean number of epochs $( \pm$ S.E. $)$ for MMN averages was not significantly different between deviants, nor were there differences in epoch numbers across placebo (frequency $=172.67 \pm 2.55$; intensity $=171.72 \pm 2.57$; consonant $=170.78 \pm 3.02$; vowel duration $=171.22 \pm 2.68$; across vowel $=171.33 \pm 2.78$ ) and ketamine conditions (frequency $=174.00 \pm 1.77$; intensity $=174.78 \pm 1.85$; consonant $=173.89 \pm 1.95$; vowel duration $=173.83 \pm 1.99$; across vowel $=174.72 \pm 1.65$ ). In contrast to the original study employing the fast multi-feature Finnish speech paradigm (Näätänen et al., 2004), which employed a mastoid reference and thus evidenced no MMN generation at temporal/posterior 


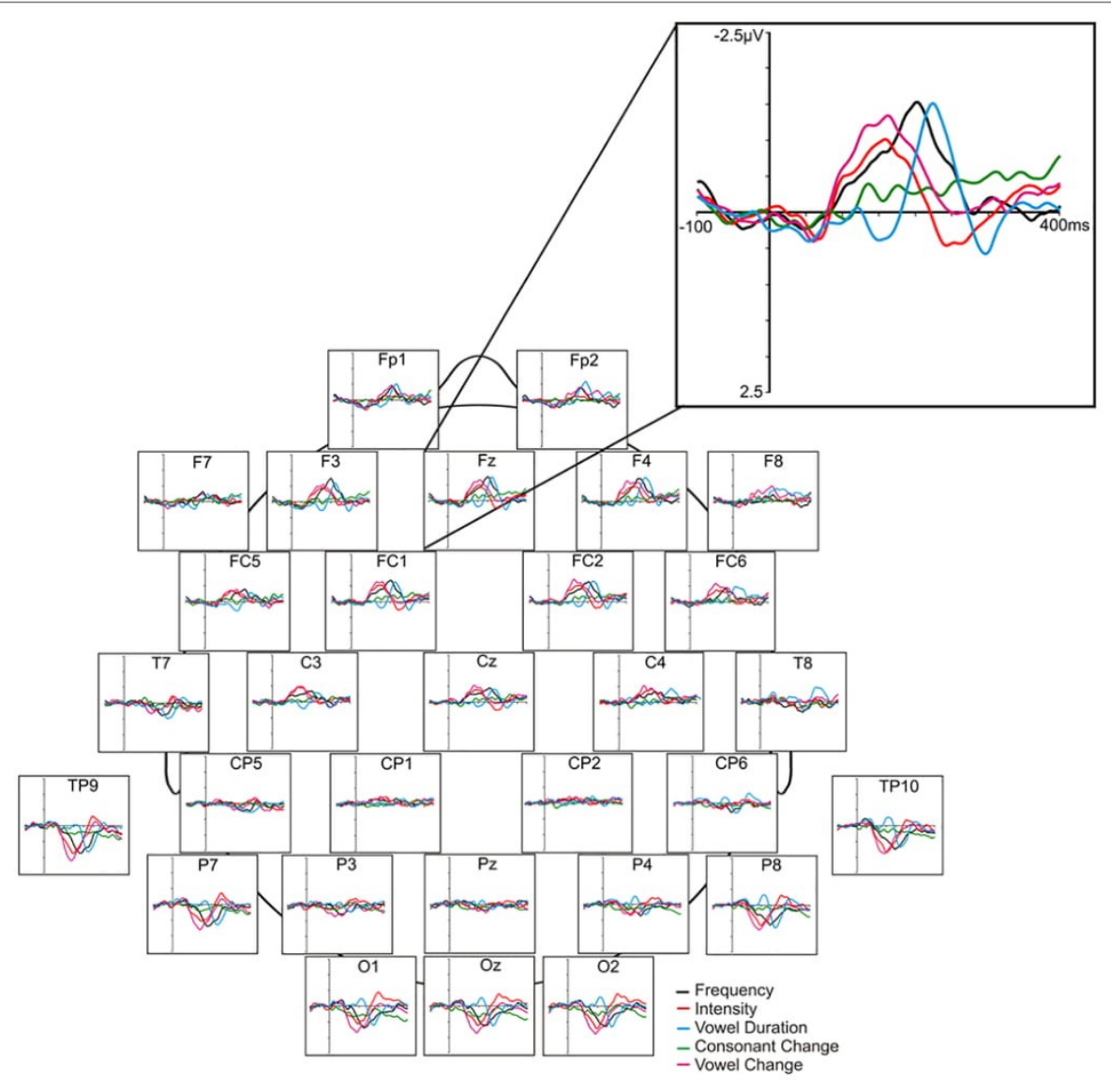

FIGURE 1 | Grand averaged ERP difference waveforms elicited in response to 5 speech deviants in the placebo condition.

sites, our use of a nose reference yielded prominent polarityinverted MMN responses in these regions, which results from the dipole generator orienting between the auditory and frontal cortices. As a secondary analysis, MMNs were also measured and analyzed at left (TP9) and right (TP10) mastoid sites. Amplitude of the obligatory $\mathrm{N} 1$ component (peak negativity between 90 and $120 \mathrm{~ms}$ ) elicited by the standard stimulus was also measured in order to clarify whether or not ketamine effects were specific to deviance processing vs. general sensory processing. Figure 1 displays the placebo grand averaged difference waveforms [including the polarity reversed MMNs and mastoid (TP9, TP10) sites] for the five speech deviants, together with the grand averaged topographic maps showing amplitude distribution of MMNs across recording sites.

\section{ERO and ERSP Processing}

For each deviant type, the average frontal ERO was obtained for the theta band at Fz, F3, and F4 sites in a manner similar to that of Hall et al. (2011). Time-varying spectral activity $(\mu \mathrm{V})$ was computed on averaged epochs (separately for each deviant) using a complex Morlet wavelet with a constant [c $=\sigma_{\mathrm{f}} / f$, where $\sigma_{\mathrm{f}}=$ the standard deviation of the centre frequency $(f)]$ value of 5 ; this was computed over the range of $1-20 \mathrm{~Hz}$ with 40 frequency steps. Evoked theta activity (3$7 \mathrm{~Hz}$ ) within time-frequency ROIs were exported for analysis ( $\pm 50 \mathrm{~ms}$ around peak MMN amplitude). ERO values were then $\ln$-transformed to ensure that the data were normally distributed for statistical analysis. Using the same methodology, theta ERO (within the MMN latency range) was quantified for the standard stimulus to determine whether ketamine effects were specific to deviance processing or generalized to all sensory processing. As background, ongoing oscillations may moderate stimulus-induced oscillatory response, an additional analysis was conducted to assess potential changes in spontaneous theta with ketamine administration. Pre-stimulus epochs of $100 \mathrm{~ms}$ duration were digitally sampled $(500 \mathrm{~Hz})$ across standard and deviant stimuli and submitted to Fast Fourier Transform (FFT) analysis for computation of ln-transformed spectral amplitude in the theta (3-7) band in the MMN latency range. Values across epochs were summed for statistical analysis.

Phase-locking factor (PLF) values were computed by first performing wavelet transformation on each epoch, and 
subtracting the standard epochs from the deviants. By using the BrainVision Analyzer "Phase Locking Factor" solution, the average time-frequency PLF values can be extracted. PLF was calculated as 1 minus the circular variance of phases, as described by Tallon-Baudry et al. (1996), and ranges from 0 (random distribution of phases) to 1 (perfect phase locking). Similarly to the ERO data, theta PLF (3-7 Hz) within time-frequency regions of interest was exported for analysis $( \pm 50 \mathrm{~ms}$ around peak MMN amplitude). PLF was also quantified for the standard stimulus and for spontaneous (pre-stimulus) activity.

\section{Brodmann Area Regions of Interest}

Intracortical current density $\left(\mathrm{A} / \mathrm{m}^{2}\right)$ measured at peak $\mathrm{MMN}$ activity (based on ERP grand averages) from predefined ROIs were computed using exact low resolution electromagnetic tomography (eLORETA, version 2081104; Pascual-Marqui, 2007; Pascual-Marqui et al., 2011). eLORETA models the cortex as a collection of voxels (6239 voxels with a spatial resolution of $7 \mathrm{~mm}$ ). Relying on the digitized Talairach atlas and the average MRI brain provided by the Montreal Neurological Institute (MNI) and a cortically restrained solution space, it calculates the non-unique "inverse" problem by computing a three dimensional distribution of intracortical source activity (with zero location error) at each voxel based on surface-level electrical signals. The original LORETA method has received considerable validation from studies using more established localization methods such as structural and functional MRI, PET, and implanted electrode recordings. The selected ROIs were based on eLORETA-defined Brodmann Areas (BA), and current density data from a single centroid representative voxel of each $\mathrm{BA}$ (the voxel closest to the center of the BA mass, which is an excellent representation of the corresponding BA) were extracted for further analysis. This included the primary (BA 41) and secondary (BA 42) auditory cortices and the three regions comprising the IFG (BAs 44, 45 , and 47 ). Current density was derived for the left and right hemispheres of each BA.

\section{Statistical Analyses}

Statistical analysis was conducted using SPSS version 23 (SPSS Inc., Chicago, IL, United States). Separate repeated measures analysis of variances (rmANOVA) for each EEG measure were carried out. The ERP amplitude, theta ERO, and theta PLF ANOVAs consisted of three within-group factors, including drug (placebo and ketamine), deviant type (frequency, intensity, vowel duration, across vowel, and consonant) and electrode site [left (F3), central (Fz), and right (F4)]. A similar but secondary set of analyses, limited to ERP amplitude, were conducted using responses derived at left (TP9) and right (TP10) temporal electrode sites. MMN latency (at $\mathrm{Fz}$ only) was analyzed with similar ANOVAs but with no site factor, while latency at temporal sites was analyzed at left and right hemispheres. Finally, for ERPs, the N100 amplitude/latency values derived from the standard stimulus were also subjected to similar rmANOVA with drug and electrode site (Fz, F3, and F4) as factors. The eLORETA-derived current density values were also analyzed using a rmANOVA and involved two within-group factors, including drug (placebo and

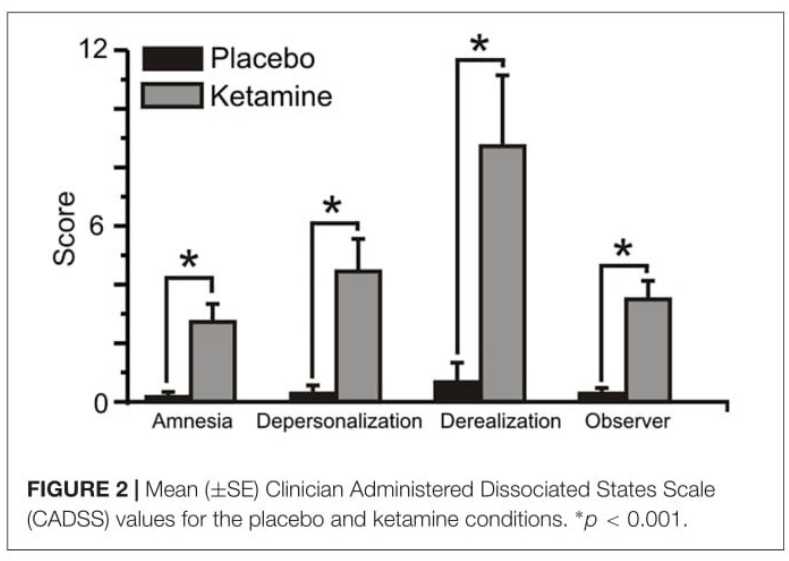

ketamine) and ROI (BA41, BA42, BA44, BA45, and BA47). Significant (Greenhouse-Geisser corrected when appropriate) effects were followed up with Bonferroni-adjusted comparisons. The CADSS subscales exhibited non-normal distributions and were analyzed using the non-parametric Wilcoxon Signed Ranks Test (WSRT).

Spearman's rho correlations were used to examine the relationship between baseline (placebo) electrophysiological measures (ERP, ERO, PLF, and BA current density) with changes in dissociate/perceptual symptoms [difference scores (ketamine - placebo) as for the CADSS sub-scales]. For ERPs (MMN), correlations were only conducted with the frontal midline recording site $(\mathrm{Fz})$ and only correlations equal/less than $P<0.01$ were considered significant in order to reduce likelihood of chance findings associated with multiple testing.

\section{RESULTS}

\section{CADSS Symptom Ratings}

Figure 2 displays the mean $( \pm S E)$ rating scores for the placebo and ketamine conditions. Relative to placebo, ketamine increased observer rated symptom scores (WSRT $=-3.40, p<0.001$ ) and for self-ratings, depersonalization (WSRT $=-3.30, p<0.001$ ), derealization (WSRT $=-3.52, p<0.0001$ ), and amnesia scores (WSRT $=-3.31, p<0.001)$ were significantly higher with ketamine than with placebo.

\section{MMN Amplitude/Latency}

Mid-frontal (Fz) grand averaged deviant-minus-standard difference waveforms for each deviant type in the multi-feature speech paradigm presented in placebo and ketamine conditions are shown in Figure 3. Significant main effects were observed for the drug condition across all linguistic sound features $(F=24.29, \mathrm{df}=1 / 17, p<0.0001)$, with ketamine reducing MMN in response to changes in syllable frequency, syllable intensity, vowel-duration, consonant change, as well as vowel change. Drug did not interact with deviant type or electrode site, but follow-up of a significant deviant effect $(F=18.17$, 

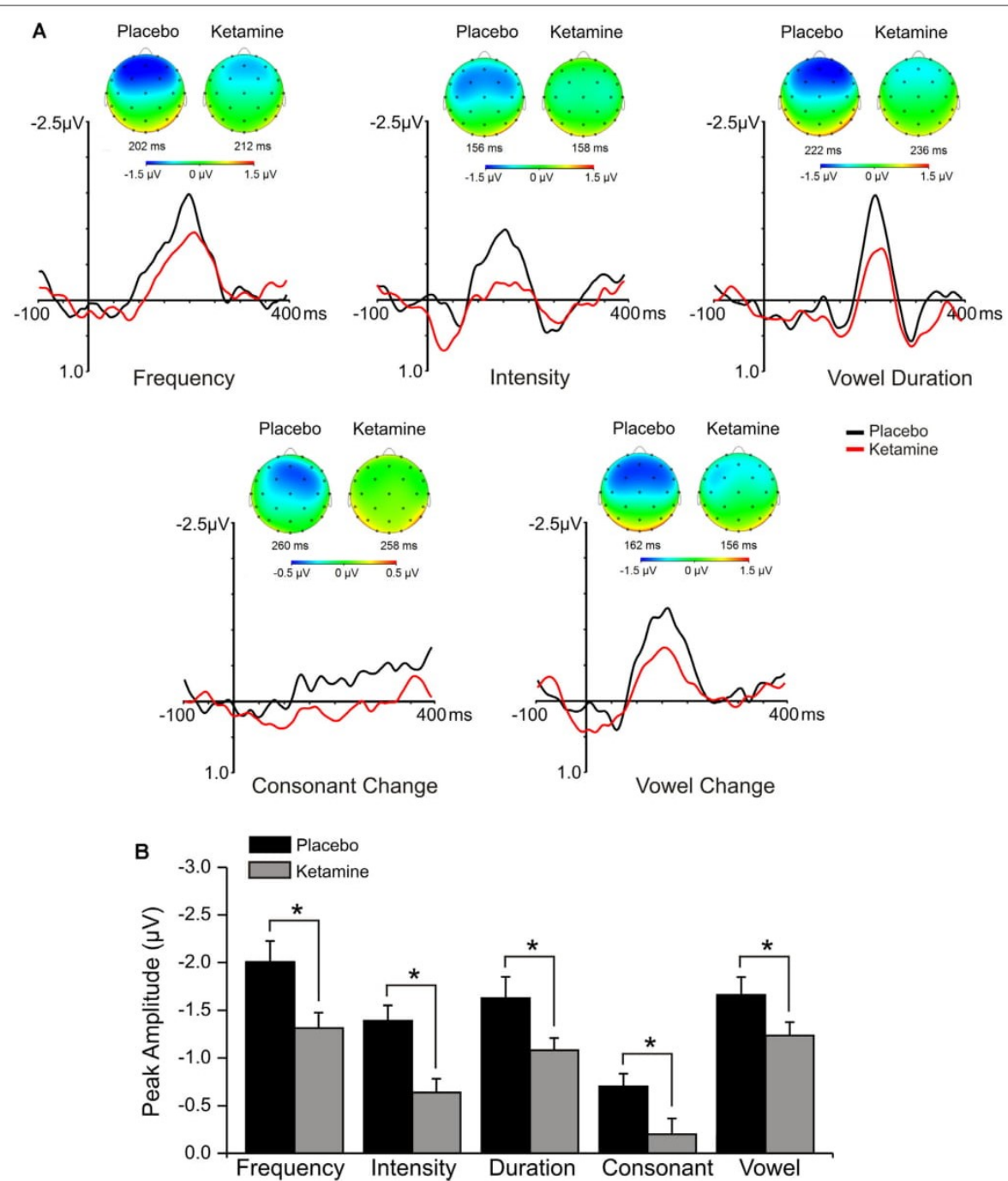

FIGURE 3 | (A) Grand averaged ERP waveforms at Fz and topographic maps generated in response to 5 speech deviants during placebo and ketamine conditions. (B) Mean ( \pm SE) peak frontal amplitudes $(\mu \mathrm{V})$ for the placebo and ketamine conditions. ${ }^{*} p<0.05$.

df $=4 / 68, p<0.0001)$ found largest MMNs with frequency, vowel duration and across vowel deviants, which showed similar amplitudes, each of which was greater than intensity $(p=0.01)$, with the latter deviant producing the smallest of the MMNs. For the significant electrode site effect $(F=8.39, \mathrm{df}=2 / 34$, $p<0.001)$, MMN amplitude at frontal midline $(\mathrm{Fz})$ was greater than both left (F3) and right (F4) frontal MMNs $(p<0.05)$, which exhibited similar amplitudes. Frontal MMN latency was not altered by ketamine.

Left (TP9) and right (TP10) temporal grand averaged deviantminus standard waveforms for each deviant type recorded during placebo and ketamine conditions are shown in Figure 4. Although not interacting with deviant type or electrode site, a significant drug effect $(F=6.17, \mathrm{df}=1 / 17, p<0.03)$ showed reduced MMN amplitudes with ketamine vs. placebo administration, particularly at TP9 sites. For the significant deviant type effect $(F=10.19$, df $=4 / 68, p<0.0001)$, the largest MMNs were equivalently elicited by intensity, frequency and across vowel deviants. Compared to all deviant types, the consonant deviant elicited the smallest MMN $(p<0.001)$. MMN elicited by the duration deviant was similar to the MMN elicited by the frequency deviant but smaller than the intensity $(p<0.04)$ and vowel duration $(p<0.007)$ MMN. Temporal MMN latencies were not affected by ketamine.

N1 amplitude/latency derived from the standard stimulus ERP frontal $(\mathrm{Fz})$ recording sites were not altered by ketamine administration $(F=0.76, \mathrm{df}=1 / 17, p=0.79)$. 
A

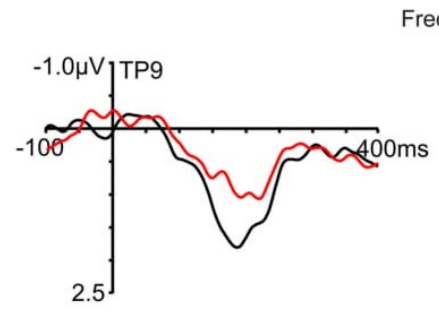

Frequency

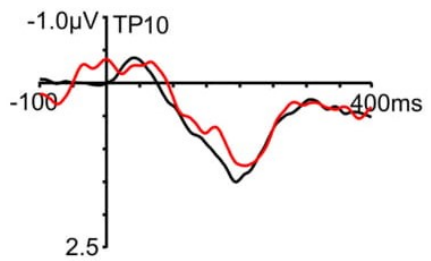

Intensity
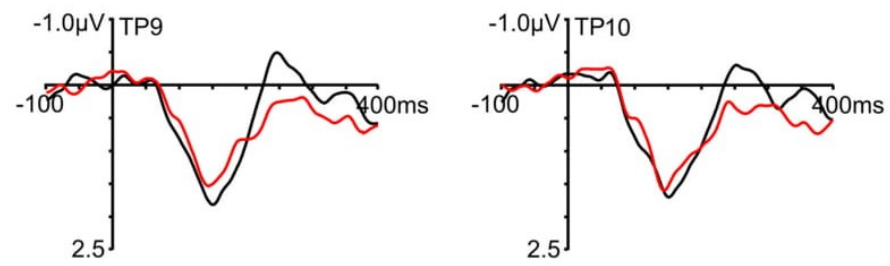

Vowel Duration
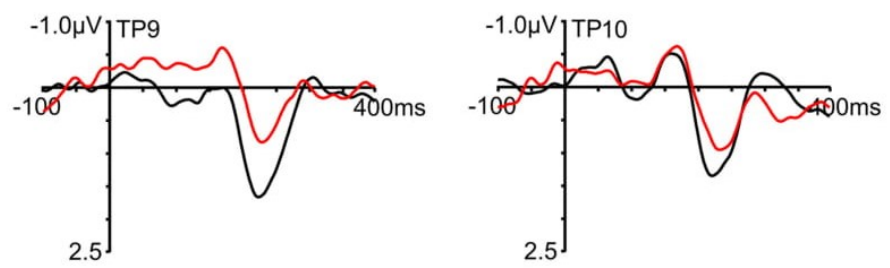

Consonant Change
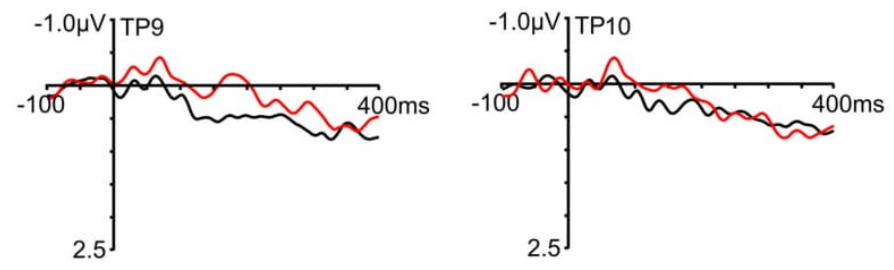

Vowel Change
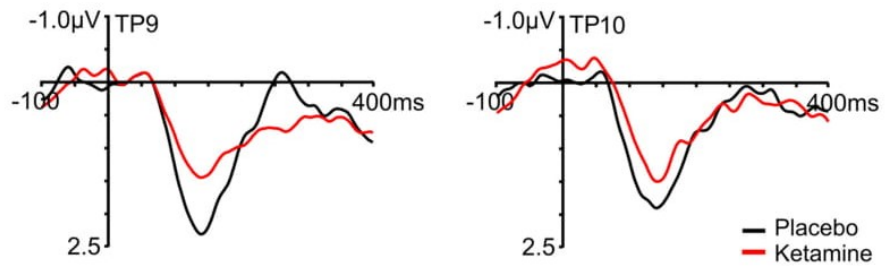

B
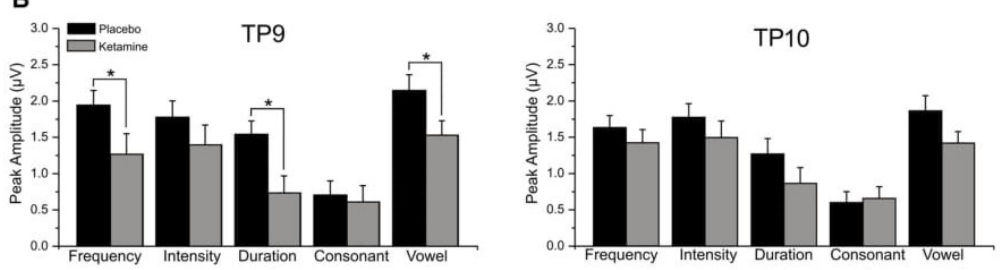

FIGURE 4 | (A) Grand averaged ERP waveforms at mastoid sites (TP9 and TP10) generated in response to 5 speech deviants during placebo and ketamine conditions. (B) Mean ( \pm SE) peak temporal amplitudes $(\mu \bigvee)$ for the placebo and ketamine conditions. ${ }^{*} p<0.05$. 

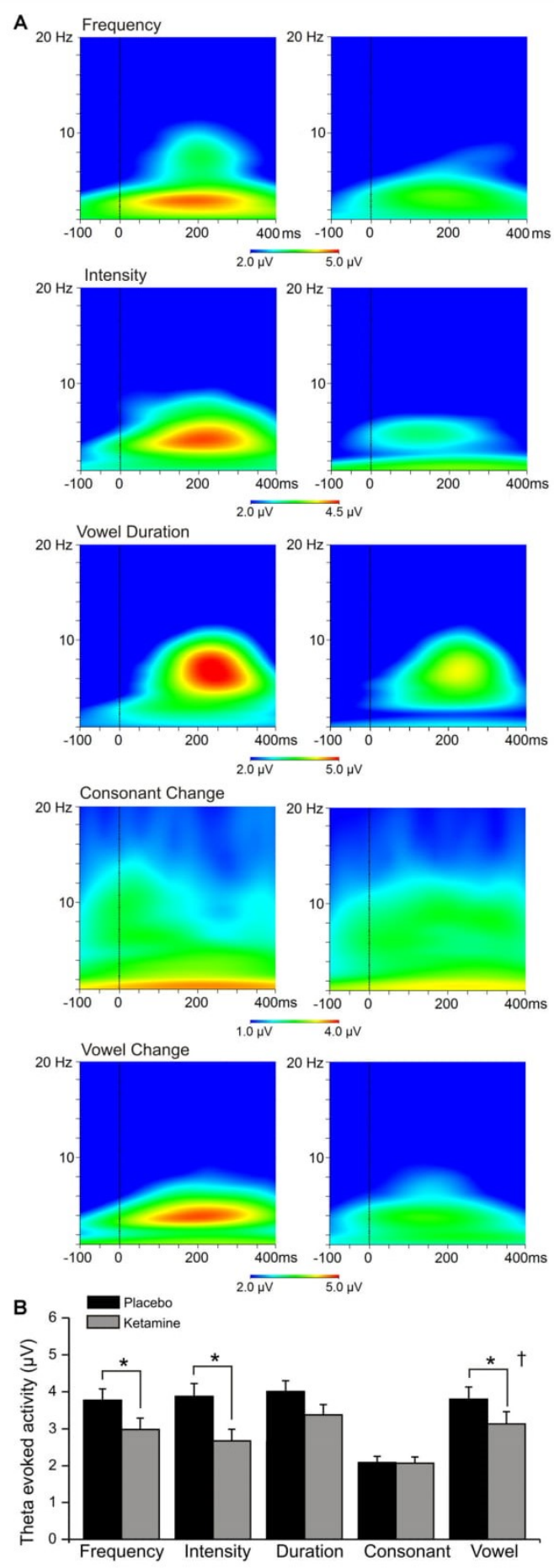

FIGURE 5 | (A) Grand averaged time frequency plots showing theta power response at the frontal midline $(\mathrm{Fz})$ site to 5 speech deviants during placebo and ketamine conditions. (B) Mean ( \pm SE) frontal theta evoked activity $(\mu \mathrm{V})$ for the placebo and ketamine conditions. ${ }^{*} p<0.05 . \dagger=$ at Fz only.
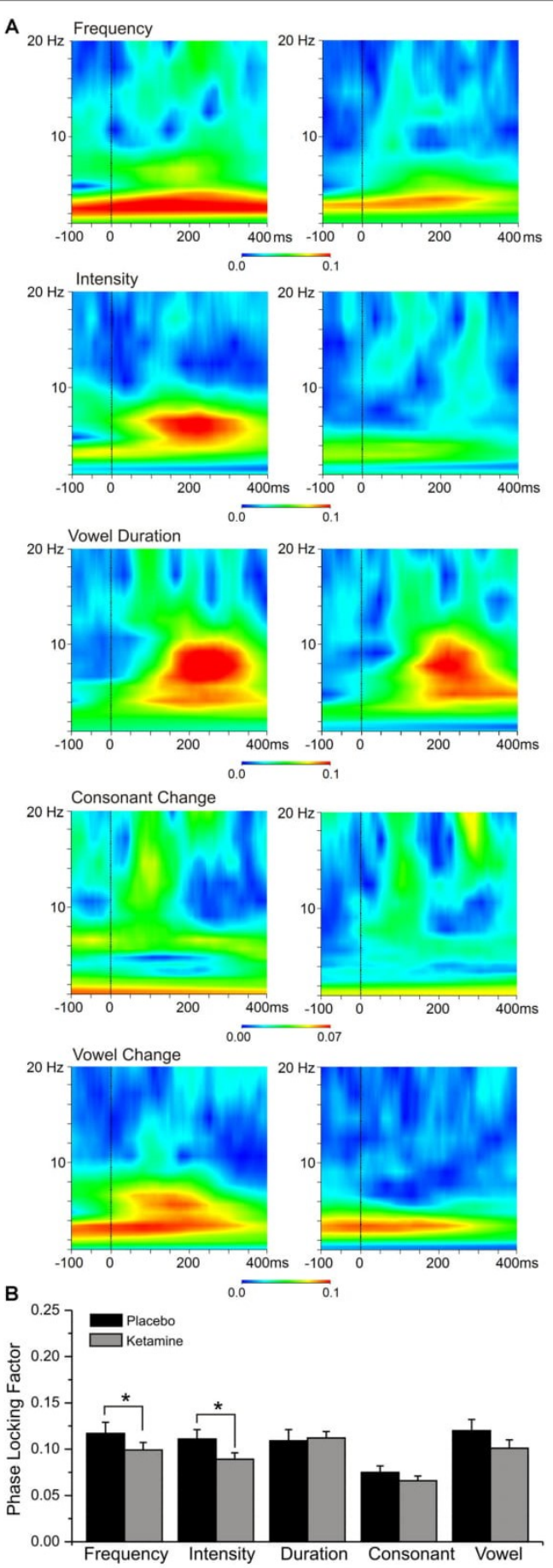

FIGURE 6 | (A) Grand averaged event-related spectral perturbation (ERSP) responses highlighting theta phase locking factor changes at the frontal midline (Fz) site to 5 speech deviants in placebo and ketamine conditions. (B) Mean $( \pm \mathrm{SE})$ frontal phase locking factor for the placebo and ketamine conditions. 


\section{Evoked Theta Power}

Figure 5 shows the grand averaged evoked (stimulus-locked) time-frequency responses to each speech deviant type during placebo and ketamine administration sessions. Significant main drug effects were exhibited $(F=21.99, \mathrm{df}=1 / 17, p<0.0001)$, but follow-up tests showed evoked theta power for frequency, intensity and vowel deviants being reduced by ketamine compared to placebo administration. Although not interacting with deviant type, follow-up of a significant deviant effect $(F=9.35, \mathrm{df}=4 / 68, p<0.001)$ found greater evoked power at midline $(\mathrm{Fz})$ compared to both left $(\mathrm{F} 3)$ and right $(\mathrm{F} 4)$ sites $(p<0.007)$. Evoked theta power elicited by the standard stimulus was not affected by ketamine. Ketamine administration did not alter ongoing spontaneous theta power derived from frontal recording sites.

\section{Phase Locking Factor}

Grand averaged PLF for each speech deviant type during placebo and ketamine conditions are shown in Figure 6. Significant overall drug effects were observed $(F=9.63, \mathrm{df}=4 / 68, p<0.0001)$ with ketamine (vs. placebo) significantly diminishing PLF in relation to frequency and intensity deviant types. A significant deviant effect $(F=8.13$, df $=4 / 68, p<0.0001)$ showed consonant PLF to be smaller compared to PLFs associated with other deviants $(p<0.02)$. Electrode site differences were also shown $(F=3.49$, df $=2 / 34, p<0.05)$, with greater PLF being observed at frontal midline (Fz) site compared to left (F3) and right (F4) frontal sites $(p<0.007)$. Theta evoked power elicited by the standard stimuli was not influenced by ketamine, nor was PLF derived from spontaneous (pre-stimulus) activity affected by ketamine.

\section{Source Current Density}

Mean $( \pm$ SE) ROI current density values for auditory and frontal cortices during placebo and ketamine conditions are displayed in Figure 7. With the exception of consonant and vowel change deviants, current density across all deviants and ROIs were significantly altered by drug administration $(F=13.03$, df $=1 / 17$, $p<0.002$ ), with ketamine reducing current density vs. placebo. Only the pAC and sAC regions failed to respond to ketamine during the processing of frequency deviants. Regions varied in current density $(F=16.94, \mathrm{df}=4 / 68, p<0.0001)$, with the highest current density being observed in the IFG (BA47) compared to other regions $(p<0.003)$ and the lowest density was in the pAC.

In a significant deviant effect $(F=3.19, \mathrm{df}=4 / 68$, $p<0.02)$, consonant change produced the lowest current density compared to other deviants $(p<0.02)$ and, although similar to vowel duration and syllable intensity/frequency deviant current densities, the across vowel deviant exhibits the highest current density values. Follow-up of a significant deviance $\mathrm{x}$ region interaction $(F=4.02, \mathrm{df}=16 / 272, p<0.0001)$, found that the comparatively lower consonant (vs. other deviants) current density was limited to the pAC $(p<0.02)$ and that for all deviants the highest current density was in the IFG (BA47), which exhibited greater density values than the pAC across deviants $(p<0.05)$, greater density values than all other regions for the vowel duration deviant $(p<0.04)$, and greater density values than BA44 $(p<0.04)$ and BA45 $(p<0.03)$ for syllable frequency, syllable intensity and across vowel deviants.

\section{Neural-CADSS Correlations}

Of the three speech MMNs, which were reduced in amplitude with ketamine administration, only the syllable intensity MMN and its associated oscillatory and source activities were related to changes in subjective symptoms elicited by ketamine administration (Figure 8). With respect to MMN itself, individuals with smaller $\mathrm{Fz}$ amplitudes during placebo experienced greater ketamine-induced increases in derealization $(r=0.631, N=18, p<0.001)$ and amnesia ratings $(r=0.594$, $N=18, p<0.009$ ). As shown in Figure 9, placebo theta PLF at Fz were negatively correlated with ketamine-induced increases in derealization $(r=-0.613, N=18, p<0.007)$ ratings.

For the LORETA-derived ROIs (Figure 10), current density in the right hemisphere pAC was inversely related to changes in amnesia $(r=-0.612, N=18, p<0.007)$ and derealization ratings $(r=-0.608, N=18, p<0.007)$ with ketamine, while current density in regions of interest of the right IFG were negatively correlated with ketamine-induced increases in selfrated derealization (BA47: $r=-0.584, N=18, p<0.01$ ) and observer-rated psychotomimetic symptoms (BA44: $r=-0.555$, $N=18, p<0.017)$.

\section{DISCUSSION}

\section{Summary of Findings}

The present study evaluated the hypothesis that the NMDA receptor system is critically involved in the early sensory processing of speech. As predicted, a sub-anesthetic dose of the NMDA antagonist, ketamine, significantly diminished frontal and temporal MMN generation in response to speech deviants. To our knowledge, this is the first report suggesting a causative relationship between NMDA receptor blockade and impaired speech MMN generation. The absence of ketamine effects on sensory ERP (N1) with a similar latency suggests that the reduction of speech MMNs in this study is not the result of a general weakening of ERP generators, but specifically involves neuronal processes generating the auditory MMN, including the MMN elicited by human speech. Supporting this, NMDA receptor antagonism reduced both evoked theta power and theta phase locking associated with diminished speech MMNs, and it attenuated current density in the auditory (pAC and $\mathrm{sAC}$ ) cortex and in IFG brain regions involved in the processing and production of speech. This is consistent with previous studies showing a contributing influence of theta oscillations, and activity in temporal and frontal brain regions in the generation of the MMN. In our healthy volunteers without a history of psychopathology, speech associated neural activity assessed in the non-drug (placebo) state was negatively correlated with ketamine-induced psychotomimetic experiences, indicating that these electrophysiological markers may reflect the functional state of the NMDA receptor-mediated system and its vulnerability to disruption by receptor antagonism. 

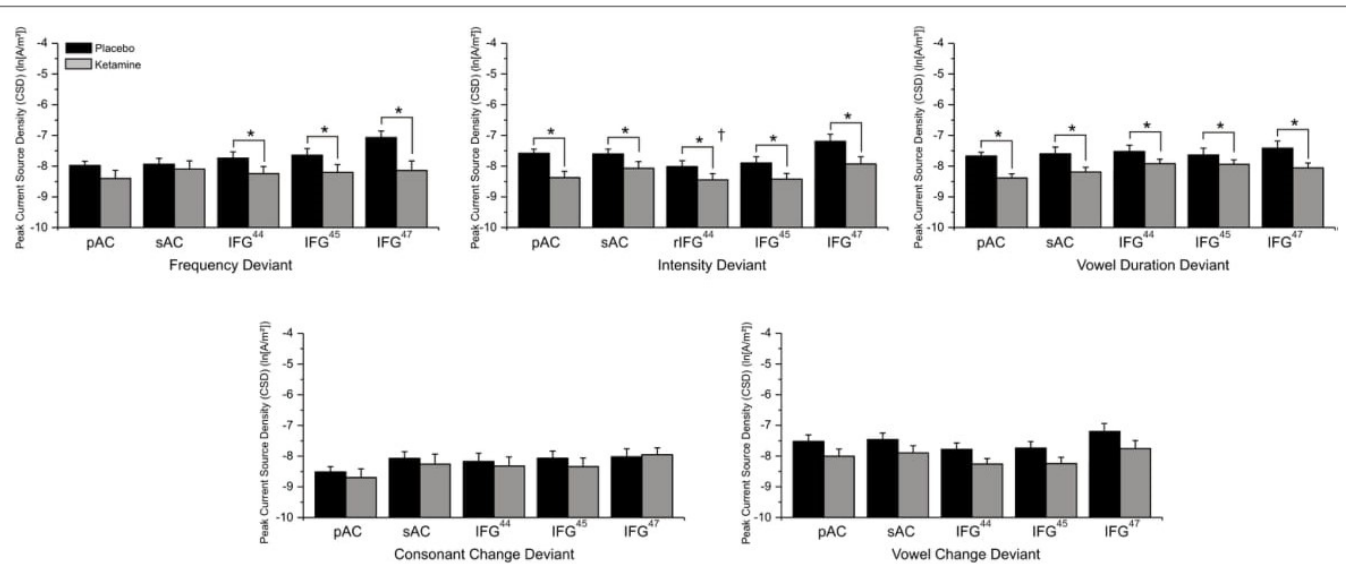

FIGURE 7 | Mean ( \pm SE) In-transformed source-localized CSD values $\left(\mathrm{A} / \mathrm{m}^{2}\right)$ of Brodmann Area $(\mathrm{BA})$ regions of interest associated with 5 speech deviants during placebo and ketamine conditions. ${ }^{*} p<0.05$ (IFG ${ }^{44}=\mathrm{BA} 44$; IFG $=\mathrm{BA} 45 ; \mathrm{IFG}^{47}=\mathrm{BA} 47$; $\dagger=$ right hemisphere effect).
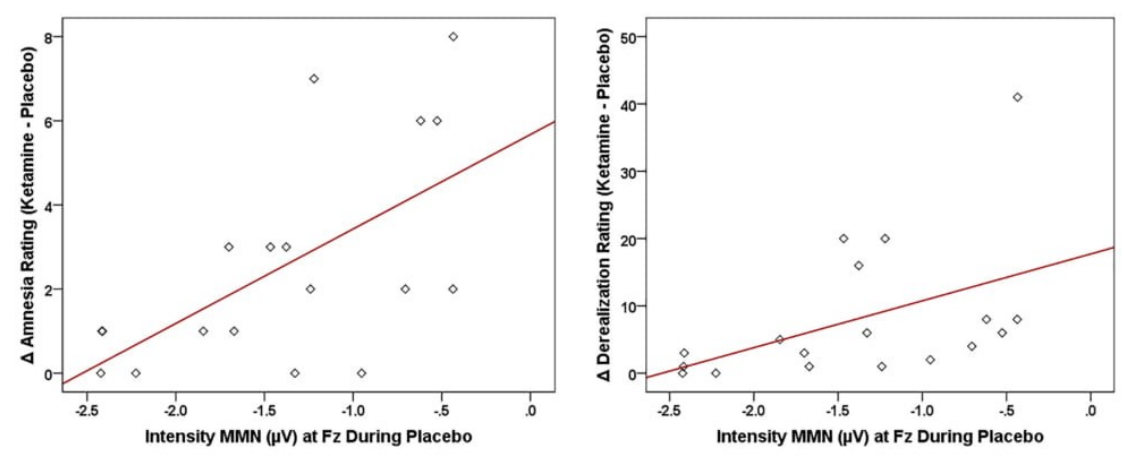

FIGURE 8 | Scatterplots showing significant relationships between ketamine-induced changes in symptom rating (derived by subtracting placebo CADSS values from ketamine CADSS values) and baseline (placebo) intensity MMN amplitude.

\section{Ketamine-MMN Effects}

Though no ketamine influence was observed on MMN latency, our MMN amplitude results with speech MMNs in healthy volunteers generally conform with meta-analyses involving simple tone stimuli in humans, which show reductions in the MMN response with ketamine (Rosburg and KreitschmannAndermahr, 2016). This effect is also reported in a range of animal models, including recordings in mice (Umbricht et al., 2005; Ehrlichman et al., 2008), rats (Tikhonravov et al., 2008), monkeys (Javitt et al., 1996; Gil-da-Costa et al., 2013), and also pigeons (Schall et al., 2015). Whereas in previous research ketamine's effects on MMN generated by simple acoustic stimuli were numerically but not statistically different for deviant type (i.e., ketamine tended to attenuate tone duration MMN amplitude more than frequency MMN amplitude on visual inspection alone, but this did not reach statistical significance), ketamine impairment of speech MMNs was observed across all deviant types at frontal and less so at temporal recordings. Although MMN amplitudes varied with speech deviance, the baseline (placebo) temporal MMN amplitude generally did not appear to moderate the presence of significant ketamine effects on MMN. For example, MMN in response to consonant change was significantly smaller than MMNs elicited by the other four but all were significantly affected by ketamine administration. The noticeably small consonant MMN, exhibiting no clear discernible onset/offset or peak within the typical MMN latency window, has been attributed, in previous work, to more difficult discriminability (vs. standard) based on behavioral discrimination tests (Kantrowitz et al., 2015).

Mismatch negativity is thought to consist of two main generators: one in the bilateral auditory cortex, underlying pre-perceptual sound change detection in the auditory cortex, which is thought to trigger the frontal cortex MMN generator, associated with the initiation of attention switch to sound change (Näätänen and Alho, 1997; Näätänen et al., 2007). Given the ketamine-induced MMN reductions at frontal and temporal recording sites and the suggestion that $\mathrm{MMN}$ generation involves 
prediction-error signaling (Todd et al., 2013), it is reasonable to speculate that in the language domain, glutamate dysregulation due to NMDA receptor hypofunction may induce problems in prediction-error generation (i.e., the $\mathrm{MMN}$ ) in a temporofrontal network circuit involved in early sensory processing, manifesting as diminished MMNs to speech deviants. Nonsignificant ketamine effects for consonant and syllable intensity deviant MMNs at temporal but not frontal cortex has potential relevance for MMN findings in schizophrenia.

\section{Ketamine-Oscillatory Effects}

In addition to our MMN observations using the conventional time-domain approach, we also adopted the approach of more recent human studies and utilized 'time-frequency' neurooscillatory analyses of electrophysiological responses as a function of underlying spectral frequency.

To our knowledge, this is the first investigation of the time-frequency signature of human MMN responses to NMDA receptor antagonist effects. Not observed with the standard stimulus, averaged evoked theta power and theta phase locking associated with speech (frequency, intensity, vowel) deviants were found to be significantly diminished by ketamine compared to placebo. Time-frequency studies have revealed the contribution of the theta frequency band in driving the neuronal generation of the $\mathrm{MMN}$ in frontal and temporal areas. Although this research shows that neural generation of the MMN response is accompanied by theta band power modulation and phase alignment, their individual contribution varies with deviant type (Lee et al., 2017) and their specific association with normal and abnormal sensory/cognitive processes is not clear. NMDA receptor antagonists have been found to reduce evoked theta power during MMN generation (Lee et al., 2018) and other task events in rodent models (Ehrlichman et al., 2009; Lazarewicz et al., 2009). Interestingly, speech-evoked MMN and theta power in healthy volunteers serve as predictors of behavioral speech

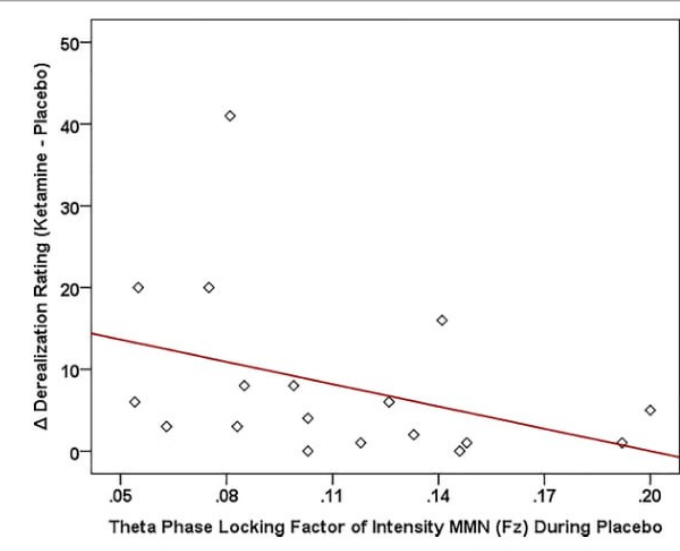

FIGURE 9 | Scatterplot showing significant relationship between ketamine-induced changes in symptom rating (derived by subtracting placebo CADSS values from ketamine CADSS values) and baseline (placebo) and theta phase locking factor (PLF). perception at the syllable and sentence level and, along with perception accuracy are reduced during noise stress (Koerrner et al., 2016), which also disrupts NMDA receptor signaling (Cui et al., 2012).

Phase resetting, which refers to the systematic adjustment of the phase of ongoing oscillations to give a consistent phase relationship to a stimulus, contributes to the generation of ERPs including the MMN. Indexed by the PLF measure of inter-trial phase variability, modulation of theta phase consistency across trials of oscillatory neural activity was significantly reduced with ketamine administration. These effects, observed in relation to frequency and intensity deviants, were concurrent with ketamine-induced reduction of speech MMNs and indicate that NMDA receptor-mediated neurotransmissions and NMDA receptor hypo-function underly normal and abnormal theta phase resetting dynamics contributing to speech MMN generation in healthy volunteers, Rodent MMN and evoked theta power impaired by NMDA receptor antagonism are mitigated by concurrent treatment with the NMDA receptor agonist glycine, suggesting that MMN generation both in time (ERP) and time-frequency (neuro-oscillatory) domain analyses, may serve as effective translational measures for revealing circuit dynamics underlying acoustic deviance detection. Compared to the auditory high frequency gamma $(40 \mathrm{~Hz})$ response, deficits of which are associated with impaired function of rapidly firing cortical parvalbumin (PV)-type expressing inhibitory interneurons in thalamocortical circuits (GonzalezBurgos et al., 2011), theta frequency rhythms are linked to interactions involving more slowly firing non-PV cells, especially somatostatin-type (SST) inhibitory interneurons in cortical circuits (Womelsdorf et al., 2014).

It is of relevance to note that ketamine-induced changes in evoked theta and PLF occurred in the absence of any changes in background, ongoing theta oscillations. Analysis of baseline (pre-stimulus) and resting EEG in healthy humans administered ketamine has been mixed and have not consistently mimicked this oscillatory abnormality (Knott et al., 2006; Kocsis et al., 2013; de la Salle et al., 2016). These present findings, observed with a sub-anesthetic dose of ketamine, suggest that impaired detection of speech deviants during NMDA receptor blockade may not be dependent on alterations in tonic cortical activity.

\section{Ketamine-Source Effects}

Neural activity in ROIs involved in the temporofrontal network implicated in the generation of the auditory MMN elicited by speech (frequency, intensity, vowel duration) change was significantly attenuated by ketamine. Depending on deviant type, intracortical current density in both temporal and frontal cortical areas was reduced concurrently with impairments in scalp-recorded $\mathrm{MMN}$ and theta oscillatory responses during detection of speech deviants. Paralleling recent LORETA observations in healthy volunteers of ketamineinduced reductions in current density of auditory, cingulate and middle frontal cortical sources of tone deviant MMNs (Thiebes et al., 2017), our present source localization findings support a role for deficient NMDA receptor-mediated neurotransmission in hypofunction of auditory and frontal 
cortical generators associated with aberrant speech deviance detection in schizophrenia.

Within the predictive coding formulation of $\mathrm{MMN}$ generation, which accommodates the findings of multiple studies showing that there are temporal and frontal cortical sources underlying MMN generation (Garrido et al., 2009b), our findings of ketamine-induced reductions in speech MMNs together with current density reductions in temporal and frontal cortical regions may indicate alterations in prediction error in low level (auditory) sources and in higher cortical levels. Additionally, they may reflect changes in temporal-frontal connections that are thought to involve NMDA receptordependent synaptic plasticity and underly MMN generation (Garrido et al., 2008, 2009a). Data from neuroimaging studies of auditory-change detection suggest that recruitment of the frontal cortex (IFG), particularly in the right hemisphere, increases with decreasing deviance. This suggests that the right IFG may engage with the auditory cortex to produce a contrast enhancing effect facilitating the detection of smaller deviances (Opitz et al., 2002; Doeller et al., 2003), or complex (e.g., language), ambiguous (e.g., Finnish stimuli), and difficult-to-detect stimulus changes. Although the typically observed sequential activation of these sources (auditory cortical preceding frontal) is compatible with the notion that the auditory cortical output is "evaluated" in some way by frontal regions (Todd et al., 2012) and is observed in response to large deviants, a reverse activation pattern (IFG activation before temporal) is observed with the processing of small deviants (Tse et al., 2013). Interestingly, strong evoked theta band synchronization between temporal and frontal regions is observed during auditory MMN generation (Hsiao et al., 2010; Choi et al., 2013).

\section{Relevance to Schizophrenia}

Ketamine-induced reductions in speech MMNs at frontal and temporal recordings mimic the impaired speech MMN generation observed in schizophrenia with EEG and magnetoencephalography (MEG) recordings (Kasai et al., 2002; Kasai, 2004; Yamasue et al., 2004; Kawakubo et al., 2006; Kawakubo et al., 2007; Fisher et al., 2008) and directly implicate deficient NMDA receptor- dependent neurotransmission in the aberrant processing of speech in this disorder. NMDA-mediated disruption of speech processing may possibly contribute to negative symptoms (such as social withdrawal) by reducing attentional switching to socially relevant auditory cues (e.g., intensity in a speaker's voice) and/or may contribute to the phenomenology of thought-disordered speech and false perceptual influences in the language system (i.e., auditory verbal hallucinations).

These MMN effects were concurrent with ketamine-induced disruptions in evoked theta power and theta phase locking and are consistent with findings linking MMN dysfunction in chronic and first episode schizophrenia primarily to an impaired theta

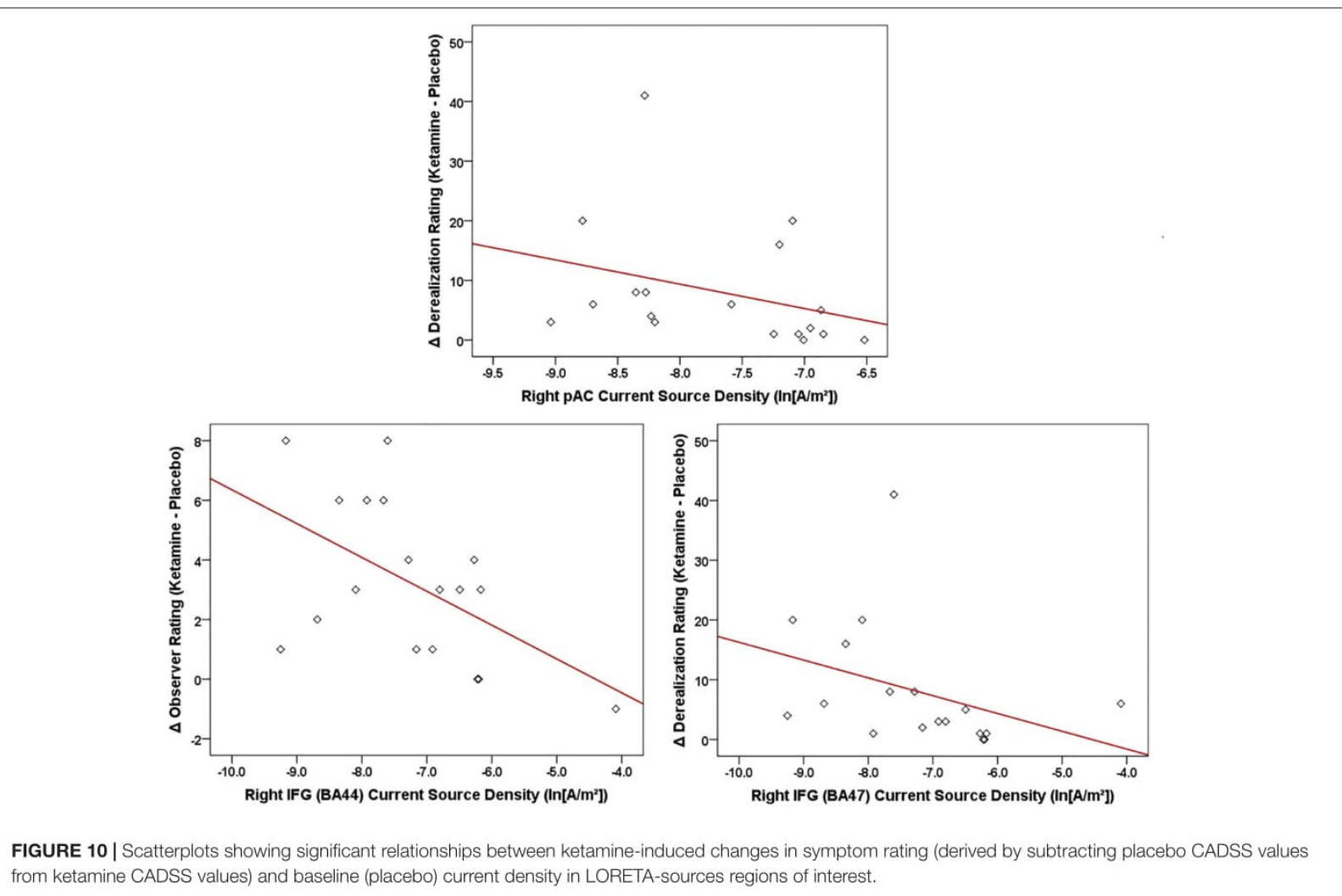


frequency response (Todd et al., 2008; Bishop and Hardiman, 2010; Moran and Hong, 2011; Choi et al., 2013; Hermann et al., 2014; Xiong et al., 2019). It has been suggested that by diminished theta power modulation and phase resetting in response to speech stimuli, may contribute to cognitive dysfunction in schizophrenia, and may be linked to the impaired interplay between cortical pyramidal neurons and circuit SSTtype GABAergic interneurons (Javitt et al., 2018).

These sensor-level neuroelectric alterations with ketamine were paralleled by reductions in intra-cortical current density in frontal and temporal ROIs, cortical areas which have also been shown by either EEG (Hirayasu et al., 1998; Kreitschmann-Andermahr et al., 1999; Pekkonen et al., 2002; Ahveninen et al., 2006; Thonnesen et al., 2008), high-density EEG (Park et al., 2002; Youn et al., 2003), fMRI (Wible et al., 2001; Kircher et al., 2004), or LORETA sourcing (Miyanishi et al., 2013; Takahashi et al., 2013; Fulham et al., 2014), to be underactivated during MMN generation. Weaker frontal/temporal circuitry has also been associated with MMN reductions to speech stimuli in schizophrenia (Thiebes et al., 2017), and based on our findings, may implicate deficient NMDA receptor-mediated neurotransmission in underlying frontal and auditory cortical generators.

The clinical findings with ketamine in healthy volunteers were as expected, with significant dissociative effects reflected in marked increases in self-rated derealization scores, as well as by increases in amnesia and observer-rated scores. Consistently reported, and co-occurring with reductions in $\mathrm{MMN}$ in previous ketamine studies, these perceptual/dissociative phenomena are part of a spectrum of transient schizophrenialike psychoactive effects (including positive and negative symptoms) produced with acute sub-anesthetic ketamine (Thiebes et al., 2017). Unrelated to baseline symptomatology, ketamine also exacerbates positive symptoms in schizophrenia patients in a way that is strikingly reminiscent of symptoms during active episodes of the disorder (Lahti et al., 2001). Although not blocked by conventional antipsychotics such as haloperidol, and only blunted by the atypical antipsychotic clozapine (Malhotra et al., 1997), clinical trials with sarcosine (a glycine reuptake inhibitor) and glycine site agonists (e.g., D-serine) have reported beneficial effects on the negative symptoms of schizophrenia (Kircher et al., 2004; Miyanishi et al., 2013) (both glycine and glutamate binding are essential for NMDA receptor activation). Active high-dose glycine attenuates MMN in healthy humans (Leung et al., 2008) but clinical trials with D-serine in schizophrenia showed acute increases in duration MMN. Further, chronic (6 weeks) increases in frequency MMN were observed, as well as improvements in positive and negative symptoms, both of which were predicted by baseline MMN amplitudes (i.e., smaller amplitudes correlated with greater clinical response) (Kantrowitz et al., 2016, 2018; Greenwood et al., 2018). Relatedly, glutathione deficiency also produces schizophrenia phenotypes including hypofunction of NMDA receptors (Steullet et al., 2006), and administration of the glutathione precursor $\mathrm{N}$-acetyl-cysteine (NAC) improved $\mathrm{MMN}$ in chronic schizophrenia (Lavoie et al., 2008) and in early psychosis (Retsa et al., 2018).

Although not implying any risk of disorder onset in our healthy volunteers, individuals varied in their susceptibility to particular psychotomimetic symptoms induced by ketamine. Individual differences in ketamineinduced effects were strongly correlated with the magnitude of $\mathrm{MMN}$ in a baseline (placebo) condition, in particular with smaller amplitudes to the intensity deviant being associated with more intense psychosis-like symptoms. This finding parallels similar observations showing baseline tone duration and tone frequency MMNs correlating with the degree of psychosis-like experiences with ketamine (Umbricht et al., 2002). Also shown with the intensity deviant following ketamine infusion, evoked theta power and theta phase locking, as well as intracortical current density in auditory (pAC) and language-related (BA44 and BA47) brain regions at baseline were inversely correlated with the degree of psychotomimetic experiences. As with previous fMRI studies, in which activity of specific symptom changes correlated with functional alterations in distinct brain regions during ketamine infusion (Deakin et al., 2008; Honey et al., 2008), selective theta oscillatory indices and regional current densities exhibited differing relationships to ketamine-induced psychotomimetic experiences, thus supporting a NMDA receptor-regulated neural basis for the varied symptoms characterizing schizophrenia. Baseline individual differences in $\mathrm{MMN}$-indexed vulnerability to psychosis may reflect genetic influences on the cortical expression of glutamate receptors, which are reduced in schizophrenia (Catts et al., 2016) and have been shown to modulate tonal (Featherstone et al., 2015) and phonetic (Kawakubo et al., 2011) MMN response.

As observed in earlier work (Stone et al., 2008), the degree of psychosis-like experiences evoked by NMDA receptor antagonists varies with the degree of NMDA receptor occupancy. As such, stronger psychotomimetic responses at the same dose of NMDA receptor antagonist reflect a greater disruption in NMDA receptor-dependant neurotransmission and, accordingly, the MMN has been interpreted as providing some information about the functional "condition" of NMDA receptor-dependent systems. This can be regarded as an indicator of the vulnerability or resiliency of the NMDA receptor system to acute perturbation by antagonists: a smaller MMN indicates a less resilient or less abundant NMDA receptor system, that is also more perturbed by NMDA receptor antagonist treatment (Umbricht et al., 2002). Similarly, speech-related MMNs and their associated theta oscillations and intracortical current densities in frontotemporal regions may serve as neural indicators of the functional state of NMDA receptormediated neurotransmission and may be useful neuroelectric tools in studies of normal and abnormal NMDA receptor functioning in humans.

The reasons why these relationships between baseline (placebo) electrophysiology and symptom changes with ketamine were limited to detection of syllable intensity deviants are not clear. Impaired sensitivity to changes in pitch and duration 
(Todd et al., 2003; Gold et al., 2012) processes known to localize to the primary auditory cortex (Tramo et al., 2002), along with deficits in sensitivity to modulation of intensity discrimination are frequently observed in schizophrenia (Bach et al., 2011). In studies of auditory emotion recognition, patients have also shown relatively greater deficit in the ability to use pitch-based versus absolute intensity-based features of speech (Leitman et al., 2010a; Gold et al., 2012). Although it is difficult to compare the degree of change across speech deviants in our study, acoustic/vocal stimuli with high cue saliency tend to increase temporal cortex activation and associated sensory-integrative functions, while with ambiguous, low salience speech stimuli, greater evaluative processes associated with inferior temporal regions are recruited (Leitman et al., 2010b). It is possible that our baseline MMNketamine response associations seen with intensity deviants may reflect the unique cortical recruitment pattern specific to changes in speech intensity and its disruption by NMDA receptor hypofunction.

\section{LIMITATIONS}

These results provide potential insight into some of the neural mechanisms underlying NMDA receptor-mediated impairments in the sensory processing of human speech, but there are limitations to the study. The participant sample was limited to young, adult (English-speaking) healthy male volunteers, who tend to exhibit greater acute vulnerability (vs. females) to some of the behavioral effects of ketamine (Morgan et al., 2006), and thus the findings do not necessarily translate to schizophrenia patients who develop NMDA receptor adaptation with increasing chronicity. Also, although their age range (early twenties) is comparable to the typical age of onset of schizophrenia, findings may not translate for speech processing in chronic patients. New language-specific neural representations (indexed by $\mathrm{MMN}$ ) can evolve for non-native speech categories (Tamminen et al., 2015), and although the $\mathrm{MMN}$ was elicited within an optimal paradigm which allowed for neural profiling across different speech features, they were recorded in response to deviations in Finnishlanguage speech sounds and similar studies relevant to the native language (English) of the test subjects are required. Subjective effects of ketamine were evaluated with an instrument emphasizing perceptual/dissociative symptoms and did not address the positive, negative or cognitive symptomatology that characterizes schizophrenia. Ketamine was administered as a single dose, with no attempt to examine dose- or time-response variations, and the design lacked an active comparator drug (e.g., midazolam) which may allow for the assessment of non-specific behavior (e.g., drowsiness), and effects unrelated to NMDA receptor blockade. Examination of EEG oscillations was limited to theta frequencies and future studies need to assess a more complete frequency spectrum from slow delta to fast gamma rhythms. Although gamma band oscillations have also been associated with depression and fast-acting antidepressants like ketamine (Fitzgerald and
Watson, 2018), they have been frequently associated with the pathophysiology of schizophrenia and associated auditory evoked response deficits (Curic et al., 2019). Moreover, relative to standard sounds, deviant sounds elicit a cascade of oscillatory modulations (beginning with gamma and followed by beta, both of which are coupled with theta) that are altered by acute ketamine administration (Gilley et al., 2017; Schuelert et al., 2018). Further, EEG source localization lacks the spatial sensitivity of other imaging methodologies such as fMRI and was limited to specific regions of interest in the temporal and inferior frontal cortex, thus, it did not include examination of a possible role for other circuits (e.g., thalamocortical and limbic) involved in sensory processing. Although the NMDA receptor is known to specifically influence activity in the central auditory system (Wang et al., 1987) and frontal/limbic and cingulate regions (Vollenweider et al., 1987; Lahti et al., 1995; Northoff et al., 2005), it is also thought to participate in widespread suppression and activation of circuit-level neural activity (Fitzgerald, 2012). Combining EEG and other imaging tools in a ketamine model and assessing activity and connectivity within and between multiple neural networks during stimulus-evoked and resting-state conditions may be a useful approach in future studies for delineating extrinsic and intrinsic neural factors mediating region-specific underpinnings of NMDA receptor-dependent speech/language deficits pertaining to schizophrenia and associated auditory hallucinatory experiences (Northoff and Qin, 2011; Northoff, 2014; Northoff, 2015; Alderson-Day et al., 2016).

\section{CONCLUSION}

To the best of our knowledge, this is the first study assessing the effects of the NMDA receptor antagonist ketamine on speech MMNs, and their associated oscillatory activities and intra-cortical current densities in healthy humans. Reductions in MMN generation and related neural activity across speech deviants indicates a general impairment in the processing of deviant speech. Together with the emergence of perceptual/dissociative symptoms, these findings generally support a glutamatergic/NMDA receptor model of schizophrenia and of language impairment in schizophrenia in particular. In this non-psychopathological sample, baseline (placebo) MMN, theta oscillations and current density in frontotemporal regions were associated with symptom response to ketamine and may serve as objective indicators of the functional state of NMDA receptor-mediated neurotransmission.

\section{ETHICS STATEMENT}

This study was fully reviewed and approved by the Research Ethics Board (REB) of the Royal Ottawa Mental Health Centre. Participants were made aware of potential adverse events, were encouraged to ask questions, and reviewed and signed an informed consent form prior to participating in the study. 
Participants were also ensured that they could stop study participation at any time for any reason without penalty.

\section{AUTHOR CONTRIBUTIONS}

VK: conception and design of the project. SdlS, JC, DS, $\mathrm{HB}$, and VI: participant screening. SdlS, JC, DS, HB, and JM: performance of the experiments. SdlS: electrophysiology

\section{REFERENCES}

Ahveninen, J., Jaaskelainen, I., Osipova, D., Huttunen, M., Ilmoniemi, R., Kaprio, J., et al. (2006). Inherited auditory-cortical dysfunction in twin pairs discordant for schizophrenia. Biol. Psychiatry 60, 612-620. doi: 10.1016/j.biopsych.2006.04.015 Alderson-Day, B., Diederen, K., Fernyhough, C., Ford, J., Horga, G., Marqulies, D., et al. (2016). Auditory hallucinations and the brain's resting-state networks: findings and methodological observations. Schizophr. Bull. 42, 1110-1123. doi: $10.1093 / \mathrm{schbul} / \mathrm{sbw} 078$

Avissar, M., and Javitt, D. (2018). Mismatch negativity: a simple and useful biomarker of $\mathrm{N}$-methyl $\mathrm{D}$-aspartate receptor (NMDAR)-type glutamate dysfunction in schizophrenia. Schizophr. Res. 191, 1-4. doi: 10.1016/j.schres. 2017.11.006

Avissar, M., Xie, S., Vail, B., Lopez-Calderon, J., Wang, Y., and Javitt, D. (2018). Meta-analysis of mismatch negativity to simple versus complex deviants in schizophrenia. Schizophr. Res. 195, 25-34. doi: 10.1016/j.schres.2017. 07.009

Bach, D., Buxtorf, K., Strik, W., Neuhoff, J., and Seifritz, I. (2011). Evidence for impaired social intensity processing in schizophrenia. Schizophr. Bull. 37, 426-431. doi: 10.1093/schbul/sbp092

Bickel, S., and Javitt, D. (2009). Neurophysiological and neurochemical animal models of schizophrenia: focus on glutamate. Behav. Brain Res. 204, 352-362. doi: 10.1016/j.bbr.2009.05.005

Bishop, D., and Hardiman, M. (2010). Measurement of mismatch negativity in individuals: a study using single-trial analysis. Psychophysiology 47, 697-705. doi: 10.1111/j.1469-8986.2009.00970.x

Bremner, J., Krystal, J., Putnam, F., Southwick, S., Marmar, C., Charney, D., et al. (1998). Measurement of dissociative states with the clinician-administered dissociative states scale (CADSS). J. Traum. Stress 11, 125-136. doi: 10.1023/A: 1024465317902

British Broadcasting Corporation, Discovery Channel (Firm), and Warner Home Video (Firm). (2002). The Blue Planet: Seas of Life. London: BBC Video.

Brown, M., and Kuperburg, G. (2015). A hierarchical generative framework of language processing: leaking language perception, interpretation and production abnormalities in schizophrenia. Front. Hum. Neurosci. 9:643. doi: $10.3389 /$ fnhum. 2015.00643

Carlsson, A., Hansson, L., Waters, N., and Carlsson, M. (1999). A glutamatergic deficiency model of schizophrenia. Br. J. Psychiatry Suppl. 37, 2-6. doi: 10.1192/ s0007125000293574

Catts, V., Lai, Y., Weickert, C., Weickert, T., and Catts, S. (2016). A quantitative review of the postmortem evidence for decreased cortical N-methyl-Daspartate receptor expression in schizophrenia: how can we link molecular abnormalities to mismatch negativity deficits? Biol. Psychol. 116, 57-67. doi: 10.1016/j.biopsycho.2015.10.013

Choi, J., Lee, J., Ko, P., Lee, G., Jung, K., and Kim, K. (2013). Fronto-temporal interactions in the theta-band during auditory deviant processing. Neurosci. Lett. 548, 120-125. doi: 10.1016/j.neulet.2013.05.079

Corcoran, C., Stoops, A., Lee, M., Martinez, A., Sehatpour, P., Dias, E., et al. (2018). Developmental trajectory of mismatch negativity and visual eventrelated potentials in healthy controls: implications for neurodevelopmental vs. neurodegenerative models of schizophrenia. Schizophr. Res. 191, 101-108. doi: 10.1016/j.schres.2017.09.047

Coyle, J., Balu, D., Benneyworth, M., Basu, A., and Roseman, A. (2010). Beyond the dopamine receptor: novel therapeutic targets for treating schizophrenia. Dialogues Clin. Neurosci. 12, 359-382. and statistical analysis. VK and SdlS: interpretation and manuscript preparation.

\section{FUNDING}

This research was supported in part by a grant from the Natural Sciences and Engineering Research Council (NSERC) of Canada to VK (NSERC No. 210572-152799-2001).

Cui, B., Wu, M., She, X., and Liu, H. (2012). Impulse noise exposure in rats causes cognitive deficits and changes in hippocampal neurotransmitter signaling and tau phosphorylation. Brain Res. 1427, 35-43. doi: 10.1016/j.brainres.2011.08.035

Curic, S., Leicht, G., Thiebes, S., Andreou, C., Polomac, N., Eichler, I., et al. (2019). Reduced auditory evoked gamma-band response and schizophrenia-like clinical symptoms under subanesthetic ketamine. Neuropsychopharmacology .

de la Salle, S., Choueiry, J., Shah, D., Bowers, H., McIntosh, J., Ilivitsky, V., et al. (2016). Effects of ketamine on resting-state EEG activity and their relationship to perceptual-dissociative symptoms in healthy humans. Front. Pharmacol. 7:348. doi: 10.3389/fphar.2016.00348

Deakin, J., Lees, J., McKie, S., Hallak, J., Williams, S., and Dursun, S. (2008). Glutamate and the neural basis of the subjective effects of ketamine. Arch. Gen. Psychiatry 65, 154-164. doi: 10.1001/archgenpsychiatry.2007.37

Doeller, C., Opitz, B., Mecklinger, A., Krick, C., Reith, W., and Schroger, E. (2003). Prefrontal cortex involvement in preattentive auditory deviance detection: neuroimaging and electrophysiological evidence. Neuroimage 20, 1270-1282. doi: 10.1016/S1053-8119(03)00389-6

Ehrlichman, R., Gandal, M., Maxwell, C., Lazarewicz, M., Finkel, L., and Siegel, S. (2009). N-methyl-D-aspartic acid receptor antagonist-induced frequency oscillations in mice recreate pattern of electrophysiological deficits in schizophrenia. Neuroscience 158, 705-712. doi: 10.1016/j.neuroscience.2008.10.031

Ehrlichman, R., Maxwell, C., Majumdar, S., and Siegel, S. (2008). Deviance-elicited changes in event-related potentials are attenuated by ketamine in mice. J. Cogn. Neurosci. 20, 1403-1404. doi: 10.1162/jocn.2008.20097

Featherstone, R., Shin, R., Kogan, J., Liang, Y., Matsumoto, M., and Siegel, S. (2015). Mice with subtle reduction of NMDA NR1 receptor subunit expression have a selective decrease in mismatch negativity: implications for schizophrenia prodromal population. Neurobiol. Dis. 73, 289-295. doi: 10.1016/j.nbd.2014.10.010

Fisher, D., Labelle, A., and Knott, V. (2008). Auditory hallucinations and the mismatch negativity: processing speech and nonspeech sounds in schizophrenia. Int. J. Psychophysiol. 70, 3-15. doi: 10.1016/j.ijpsycho.2008.04.001

Fitzgerald, P. (2012). The NMDA receptor may participate in widespread suppression of circuit level neural activity, in addition to a similarily prominent role in circuit level activation. Behav. Brain Res. 230, 291-298. doi: 10.1016/j. bbr.2012.01.057

Fitzgerald, P., and Watson, B. (2018). Gamma oscillations as a biomarker for major depression: an emerging topic. Transl. Psychiatry 8:177. doi: 10.1038/s41398018-0239-y

Fuentemilla, L., Marco-Pollares, J., Munte, T., and Grau, C. (2008). Theta EEG oscillatory activity and auditory change detection. Brain Res. 1220, 93-101. doi: 10.1016/j.brainres.2007.07.079

Fulham, W., Michie, P., Ward, P., Rasser, P., Todd, J., Johnson, P., et al. (2014). Mismatch negativity in recent-onset and chronic schizophrenia: a current source density analysis. PLoS One 9:e100221. doi: 10.1371/journal.pone. 0100221

Garrido, M., Friston, K., Kiebel, S., Stephan, K., Baldeweg, T., and Kilner, J. (2008). The functional anatomy of the MMN: a DCM study of the coving paradigm. Neuroimage 42, 936-944. doi: 10.1016/j.neuroimage.2008.05.018

Garrido, M., Kilner, J., Kiebel, S., and Friston, K. (2009a). Dynamic causal modeling of the response to frequency deviants. J. Neurophysiol. 101, 2620-2631. doi: 10.1152/jn. 90291.2008 
Garrido, M., Kilner, J., Stephan, K., and Friston, S. (2009b). The mismatch negativity: a review of underlying mechanisms. Clin. Neurophysiol. 120, 453463. doi: 10.1016/j.clinph.2008.11.029

Gil-da-Costa, R., Stoner, G., Fung, R., and Albright, T. (2013). Nonhuman primate model of schizophrenia using a noninvasive EEG method. Proc. Natl. Acad. Sci. U.S.A. 120, 15425-15430. doi: 10.1073/pnas.13122 64110

Gilley, P., Uhler, K., Watson, K., and Yoshinaga-Itano, C. (2017). Spectraltemporal EEG dynamics of speech discrimination processing in infants during sleep. BMC Neurosci. 18:34. doi: 10.1186/s12868-0170353-4

Gold, R., Butler, P., Revheim, N., Leitman, D., Hansen, J., Gur, R., et al. (2012). Auditory emotion recognition impairments in schizophrenia: relationship to acoustic features and cognition. J. Psychiatry 169, 424-432. doi: 10.1176/appi. ajp. 2011.11081230

Gonzalez-Burgos, G., Fish, K., and Lewis, D. (2011). GABA neuron alternations, cortical circuit dysfunction and cognitive deficits in schizophrenia. Neural. Plast. 2011:723184. doi: 10.1155/2011/723184

Gratton, G., Coles, M., and Donchin, E. (1983). A new method for off-line removal of ocular artifact. Electroencephalogr. Clin. Neurophysiol. 55, 468-484. doi: 10. 1016/0013-4694(83)90135-9

Green, M. (2006). Impact of cognitive and social cognitive impairment on functional outcomes in patients with schizophrenia. J. Clin. Psychiatry 77(Suppl. 2), 2-8. doi: 10.4088/JCP.14074sulc.02

Greenwood, L., Leung, S., Michie, P., Green, A., Nathan, P., Fitzgerald, P., et al. (2018). The effects of glycine on auditory mismatch negativity in schizophrenia. Schizophr. Res. 191, 61-69. doi: 10.1016/j.schres.2017.05.031

Hall, M., Taylor, G., Salisbury, D., and Levy, D. (2011). Sensory gating eventrelated potentials and oscillations in schizophrenia patients and their unaffected relatives. Schizophr. Bull. 37, 1187-1199. doi: 10.1093/schbul/sbq027

Harms, L. (2016). Mismatch responses and deviance detection in $\mathrm{N}$-methylD-aspartate (n.d.) receptor hypofunction and developmental models of schizophrenia. Biol. Psychol. 116, 75-81. doi: 10.1016/j.biopsycho.2015.06.015

Hermann, C., Rach, S., Vosskuhl, J., and Strüber, D. (2014). Time-frequency analysis of event-related potentials: a brief tutorial. Brain Topogr. 27, 438-450. doi: 10.1007/s10548-013-0327-5

Hirayasu, Y., Potts, G., O'Donnell, B., Kwon, J., Arakaki, H., Akdag, S., et al. (1998). Auditory mismatch negativity in schizophrenia: topographic evaluation with a high-density recording montage. Am. J. Psychiatry 155, 1281-1284. doi: 10.1176/ajp.155.9.1281

Honey, G., Corlett, P., Absalom, A., Lee, M., Pomarol-Clotet, E., Murray, G., et al. (2008). Individual differences in psychotic effects of ketamine are predicted by brain function measured under placebo. J. Neurosci. 28, 6295-6303. doi: 10.1523/JNEUROSCI.0910-08.2008

Hong, L., Moran, L., Du, X., O’Donnell, P., and Summerfelt, A. (2012). Mismatch negativity and low frequency oscillations in schizophrenia families. Clin. Neurophysiol. 123, 1980-1988. doi: 10.1016/j.clinph.2012.03.011

Hsiao, F., Cheng, C., Liao, K., and Lin, Y. (2010). Cortico-cortical phase synchrony in auditory mismatch processing. Biol. Psychol. 84, 336-345. doi: 10.1016/j. biopsycho.2010.03.019

Hsiao, F., Wu, L., and Yung-Yang, L. (2009). Theta oscillation during auditory change detection: an MEG study. Biol. Psychol. 81, 58-66. doi: 10.1016/j. biopsycho.2009.01.007

Hunt, M., and Kasicki, S. (2013). A systematic review of the effects of NMDA receptor antagonists on oscillatory activity recorded in vivo. J. Psychopharmacol. 27, 972-986. doi: 10.1177/0269881113495117

Javitt, D. (2000). Intercortical mechanisms of mismatch negativity dysfunction in schizophrenia. Audiol. Neurootol. 5, 207-215. doi: 10.1159/0000 13882

Javitt, D. (2009a). Sensory processing in schizophrenia: neither simple nor intact. Schizophr. Bull. 35, 1059-1064. doi: 10.1093/schbul/sbp110

Javitt, D. (2009b). When doors of perception close: bottom-up models of disrupted cognition in schizophrenia. Clin. Psychol. 5, 249-275. doi: 10.1146/annurev. clinpsy.032408.153502

Javitt, D. (2015). Neurophysiological models for new treatment development in schizophrenia: early sensory approaches. Ann. N. Y. Acad. Sci. 1344, 92-104. doi: $10.1111 /$ nyas. 12689
Javitt, D., and Freedman, R. (2015). Sensory processing dysfunction in the personal experience and neuronal machinery of schizophrenia. Am. J. Psychiatry 172, 17-31. doi: 10.1176/appi.ajp.2014.13121691

Javitt, D., Lee, M., Kantrowitz, J., and Martinez, A. (2018). Mismatch negativity as a biomarker of theta band oscillatory dysfunction in schizophrenia. Schizophr. Res. 191, 51-60. doi: 10.1016/j.schres.2017.06.023

Javitt, D., Spencer, K., Thaker, G., Winterer, G., and Hajós, M. (2008). Neurophysiological biomarkers for drug development in schizophrenia. Nat. Rev. Drug Discov. 7, 68-83. doi: 10.1038/nrd2463

Javitt, D., Steinschneider, M., Schroeder, C., and Arezzo, J. (1996). Role of cortical $\mathrm{N}$-methyl-D-aspartate receptors in auditory sensory memory and mismatch negativity generation: implications for schizophrenia. Proc. Natl. Acad. Sci. U.S.A. 93, 11962-11967. doi: 10.1073/pnas.93.21.11962

Javitt, D., and Sweet, R. (2015). Auditory dysfunction in schizophrenia: integrating clinical and basic features. Nat. Rev. Neurosci. 16, 535-550. doi: 10.1038/ nrn4002

Javitt, D., Zukin, S., Heresco-Levy, U., and Umbricht, D. (2012). Has an angel shown the way? Etiological and therapeutic amplifications of the PCP/NMDA model of schizophrenia. Schizophr. Bull. 38, 958-966. doi: 10.1093/schbul/ sbs069

Jobert, M., Wilson, F., Ruigt, G., Brunovsky, M., Prichep, L., and Drinkenburg, W. (2012). Guidelines for the recording and evaluation of pharmaco-EEG data in man: the international pharmaco-EEG society (IPEG). Neuropsychobiology 66, 201-220. doi: 10.1159/000343478

Kantrowitz, J., Epstein, M., Beggel, O., Rohrig, S., Lehrfeld, J., Revheim, N., et al. (2016). Neurophysiological mechanisms of cortical plasticity impairment in schizophrenia and modulation by the NMDA receptor agonist D-serine. Brain 139, 3281-3295. doi: 10.1093/brain/aww262

Kantrowitz, J., Epstein, M., Lee, M., Lehrfeld, N., Nolan, K., Shope, C., et al. (2018). Improvement in mismatch negativity generation during $\mathrm{d}$-serine treatment in schizophrenia: correlation with symptoms. Schizophr. Res. 191, 70-79. doi: 10.1016/j.schres.2017.02.027

Kantrowitz, J., Hoptman, M., Leitman, D., Moreno-Ortoga, M., Lehrfeld, N., Dias, E., et al. (2015). Neural substrates of auditory emotion recognition deficits in schizophrenia. J. Neurosci. 35, 14909-14921. doi: 10.1523/JNEUROSCI.460314.2015

Kantrowitz, J., and Javitt, D. (2010). N-methyl-D-aspartate (n.d.) receptor dysfunction or dysregulation: the final common pathway on the road to schizophrenia? Brain Res. Bull. 30, 108-121. doi: 10.1016/j.brainresbull.2010. 04.006

Kasai, K. (2004). Structural and functional abnormalities of the auditory cortex in schizophrenia. Int. Congr. Ser. 1270, 50-55. doi: 10.1016/j.ics.2004.04.052

Kasai, K., Nakagome, K., Itoh, K., Koshida, I., Iwanami, A., Fukuda, M., et al. (2002). Impaired cortical network for preattentive detection of change in speech sounds in schizophrenia: a high-resolution event-related potential study. Am. J. Psychiatry 159:4. doi: 10.1176/appi.ajp.159.4.546

Kaser, M., Soltesz, F., Lawrence, P., Miller, S., Dodds, C., Croft, R., et al. (2013). Oscillatory underpinnings of mismatch negativity and their relationship with cognitive function in patients with schizophrenia. PLoS One 8:e83255. doi: 10.1371 /journal.pone.0083255

Kawakubo, Y., Kamco, S., Nose, T., Iwanami, A., Nakagome, K., Fukuda, M., et al. (2007). Phonetic mismatch negativity predicts social skills acquisition in schizophrenia. Psychiatry Res. 152, 261-265. doi: 10.1016/j.psychres.2006.02.010

Kawakubo, Y., Kasai, K., Kudo, N., Rogers, M., Nakagome, K., Itoh, K., et al. (2006). Phonetic mismatch negativity predicts verbal memory deficits in schizophrenia. Neuroreport 17, 1043-1046. doi: 10.1097/01.wnr.0000221828.10846.ba

Kawakubo, Y., Suga, M., Tochigi, M., Yumoto, M., Itoh, K., Sasaki, T., et al. (2011). Effects of metabotropic glutamate receptor 3 genotype on phonetic mismatch negativity. PLoS One 6:e24929. doi: 10.1371/journal.pone.0024929

Kircher, T., Rapp, A., Grodd, W., Buchkremer, G., Weiskopf, N., Lutzenburger, W., et al. (2004). Mismatch negativity response in schizophrenia: a combined fMRI and whole-head MEG study. Am. J. Psychiatry 161, 294-304. doi: 10.1176/appi. ajp.161.2.294

Kirino, E. (2017). Mismatch negativity correlates with delta and theta EEG power in schizophrenia. Int. J. Neurosci. 17, 1257-1279. doi: 10.1080/ 00207450600936635 
Knott, V. (2000). Quantitative EEG methods and measures in human psychopharmacological research. Hum. Psychopharmacol. 15, 479-498. doi: 10. 1002/1099-1077(200010)15:7<479::AID-HUP206<3.0.CO;2-5

Knott, V., McIntosh, J., Millar, A., Fisher, D., Villeneuve, C., and Ilivitsky, V. (2006). Nicotine and smoker status moderate brain electric and mood activation induced by ketamine, and $\mathrm{N}$-methyl-D-aspartate receptor antagonist. Pharmacol. Biochem. Behav. 85, 228-242. doi: 10.1016/j.pbb.2006.08.005

Ko, D., Kwon, S., Lee, G., Im, H., Kim, H., and Jung, K. (2012). Theta oscillation related to the auditory discrimination process in mismatch negativity: oddball versus control paradigm. J. Clin. Neurosci. 8, 35-42. doi: 10.3988/jen.2012.8.1.35

Kocsis, B., Brown, R., McCarley, R., and Hajos, M. (2013). Impact of ketamine on neuronal network dynamics: translational modelling of schizophrenia-relevant deficits. CNS Neurosci. Ther. 19, 437-447. doi: 10.1111/cns.12081

Koerrner, T., Zhang, Y., Nelson, P., Wang, B., and Zou, N. (2016). Neural indices of phonemic discrimination and sentence-level intelligibility in quiet and noise: a mismatch negativity study. Hear. Res. 339, 40-49. doi: 10.1016/j.heares.2017. 04.009

Kreitschmann-Andermahr, I., Rosburg, T., Mejer, T., Volz, H., Nowak, H. and Saver, H. (1999). Impaired sensory processing in male patients with schizophrenia: a magnetoencephalographic study of auditory mismatch detection. Schizophr. Res. 35, 121-129. doi: 10.1016/s0920-9964(98)00115-7

Krystal, J., D'Souza, D., Karper, L., Bennett, A., Abi-Dargham, A., Abi-Saad, D., et al. (1999). Interactive effects of subanesthetic ketamine and haloperidol in healthy humans. Psychopharmacoloy (Berl.) 45, 193-204. doi: 10.1007/ s002130051049

Lahti, A., Holcomb, H., Medoff, D., and Tamminga, C. (1995). Ketamine activates psychosis and alters limbic blood flow in schizophrenia. Neuroreport 6, 869872. doi: 10.1097/00001756-199504190-00011

Lahti, A., Weiler, M., Michaelidis, T., Parweni, A., and Tamminga, C. (2001). Effects of ketamine in normal and schizophrenic volunteers. Neuropsychopharmacoloy 25, 455-467. doi: 10.1016/S0893-133X(01)00243-3

Lavoie, S., Murray, M., Deppen, P., Knyazeva, M., Berk, M., Boulat, O., et al. (2008). Glutathione precursor, $\mathrm{N}$-acetyl-cysteine, improves mismatch negativity in schizophrenia patients. Neuropsychopharmacology 33, 2187-2199. doi: 10.1038/ sj.npp. 1301624

Lazarewicz, M., Ehrlichman, R., Maxwell, C., Gandal, M., Finkel, L., and Siegel, S. (2009). Ketamine modulates theta and gamma oscillations. J. Cog. Neurosci. 22, 1452-1464. doi: 10.1162 /jocn.2009.21305

Lee, M., Balla, A., Sershen, H., Sehatpour, P., Lakatos, P., and Javitt, D. (2018). Rodent mismatch negativity/theta neuro-oscillatory response as a translational neurophysiological biomarker for $\mathrm{N}$-methyl-D-aspartate receptor-based new treatment development in schizophrenia. Neuropsychopharmacology 43, 571-582. doi: $10.1038 /$ npp. 2017.176

Lee, M., Sehatpour, P., Hoptman, M., Lakatos, P., Dias, E., Kantrowitz, J., et al. (2017). Neural mechanisms of mismatch negativity (MMN) dysfunction in schizophrenia. Mol. Psychiatry 22, 1585-1593. doi: 10.1038/mp.2017.3

Leitman, D., Laukka, P., Joslin, P., Saccente, E., Butler, P., and Javitt, D. (2010a). Getting the cue: sensory contributions to auditory emotion recognition impairments in schizophrenia. Schizophr. Bull. 36, 545-556. doi: 10.1093/ schbul/sbn115

Leitman, D., Wolf, D., Ragland, J., Laukka, P., Loughead, J., Valdez, J., et al. (2010b). "It's not what you say, but how you say it": a reciprocal temporo-frontal network for affective prosody. Front. Neurosci. 4:19. doi: 10.3389/fnhum.2010. 00019

Leitman, D., Schatpour, P., Higgins, B., Foxe, J., and Silipo, G. (2010). Sensory deficits and distributed hierarchical dysfunction in schizophrenia. Am.J. Psychiatry 167, 818-827. doi: 10.1176/appi.ajp.2010.09030338

Leung, S., Croft, R., O'Neill, B., and Nathan, P. (2008). Acute high-dose glycine attenuates mismatch negativity (MMN) in healthy human controls. Psychopharmacology 196, 451-460. doi: 10.1007/s00213-007-0976-8

Malhotra, A., Adler, C., Kinnison, S., Elman, I., Pickar, D., and Breier, A. (1997). Clozapine blunts N-methyl-D-aspartate antagonist-induced psychosis: a study with ketamine. Biol. Psychiatry 42, 664-668. doi: 10.1016/s0006-3223(96) 00546-x

Maxwell, M. (1992). Family Interview for Genetic Studies (FIGS): Manual for FIGS. Clinical Neurogenetics Branch, Intramural Research Program. Bethesda, MD National Institute of Mental Health.
Miyanishi, T., Sumiyoshi, T., Higuchi, Y., Seo, T., and Suzuki, M. (2013). LORETA current source density for duration mismatch negativity and neuropsychological assessment in early schizophrenia. PLoS One 8:e61152. doi: 10.1371 /journal.pone.0061152

Moghaddam, R., and Javitt, D. (2012). From revolution to evolution: the glutamate hypothesis of schizophrenia and its implications for treatment. Neuropsychopharmacology 37, 4-15. doi: 10.1038/npp.2011.181

Moghaddam, R., and Krystal, J. (2012). Capturing the angel in "angel dust": twenty years of translational neuroscience studies of NMDA receptor antagonists in animals and humans. Schizophr. Bull. 38, 942-949. doi: 10.1093/schbul/sb s075

Moran, L., and Hong, E. (2011). High and low frequency neural oscillations in schizophrenia. Schizophr. Bull. 37, 659-663. doi: 10.1093/schbul/sbr056

Morgan, C., Mofeez, A., Brandner, B., Bromley, L., and Curran, H. (2004). Acute effects of ketamine on memory systems and psychotic symptoms in healthy volunteers. Neuropsychopharmacology 29, 208-218. doi: 10.1038/sj.npp. 1300342

Morgan, C., Perry, E., Cho, H., Krystal, J., and D’Souza, D. (2006). Greater vulnerability to the amnestic effects of ketamine in males. Psychopharmacology 187, 405-414. doi: 10.1007/s00213-006-0409-0

Näätänen, R. (1999). Phoneme representations of the human brain as reflected by event-related potentials. Electroencephalogr. Clin. Neurophysiol. Suppl. 49, 170-173.

Näätänen, R., and Alho, K. (1997). Mismatch negativity - The measure for central sound representation accuracy. Audiol. Neurootol. 2, 341-353. doi: 10.1159/ 000259255

Näätänen, R., and Kakkonen, S. (2009). Central auditory dysfunction in schizophrenia as revealed by the mismatch negativity (MMN) and its magnetic equivalent MMNs: a review. Int. J. Neuropsychopharmacol. 12, 125-135. doi: $10.1017 /$ S1461145708009322

Näätänen, R., Paavilainen, P., Rinne, T., and Alho, K. (2007). The mismatch negativity (MMN) is basic research of central auditory processing: a review. Clin. Neurophysiol. 118, 2544-2590. doi: 10.1016/j.clinph.2007. 04.026

Näätänen, R., Pakarinen, S., Rinne, T., and Takegata, R. (2004). The mismatch negativity (MMN)- towards the optimal paradigm. Clin. Neurophysiol. 115, 140-144. doi: 10.1016/j.clinph.2003.04.001

Näätänen, R., Todd, J., and Schall, U. (2015). Mismatch negativity (MMN) as biomarker predicting psychosis in clinically at-risk individuals. Biol. Psychiatry 116, 36-40. doi: 10.1016/j.biopsycho.2015.10.010

Northoff, G. (2014). Are auditory hallucinations related to the brain's resting state activity? A "neurophenomenal resting state" hypothesis. Clin. Psychopharmacol. Neurosci. 12, 189-195. doi: 10.9758/cpn.2014.12.3.189

Northoff, G. (2015). Resting state activity and the "stream of consciousness" in schizophrenia - Neurophenomenal hypotheses. Schizophr. Bull. 41, 280-290. doi: $10.1093 /$ schbul/sbul16

Northoff, G., and Qin, P. (2011). How can the brain's resting state activity generate hallucinations? A "resting state hypothesis" of auditory verbal hallucinations. Schizophr. Res. 127, 202-214. doi: 10.1016/j.schres.2010.11.009

Northoff, G., Richter, A., Bermpohl, F., Grimm, S., and Martin, V. (2005). NMDA hypofunction in the posterior cingulate as a model for schizophrenia: an exploratory ketamine administration study in fMRI. Schizophr. Res. 72, 235248. doi: 10.1016/j.schres.2004.04.009

Opitz, B., Rinne, T., Mecklinger, A., von Cramon, D., and Schroger, E. (2002) Differential contribution of frontal and temporal cortices to auditory change detection: fMRI and ERP results. Neuroimage 15, 167-174. doi: 10.1006/nimg. 2001.0970

Paavilainen, P. (2013). The mismatch-negativity (MMN) component of the auditory event-related potential to violations of abstract regularities: a review. Int. J. Psychophysiol. 88, 109-123. doi: 10.1016/j.ijpsycho.2013. 03.015

Pakarinen, S., Lovio, R., Huotilaninen, M., Alku, P., Näätänen, R., and Kujala, T. (2009). Fast multi-feature paradigm for recording several mismatch negativities (MMNs) to phonetic and acoustic changes in speech sounds. Biol. Psychol. 82, 219-226. doi: 10.1016/j.biopsycho.2009.07.008

Park, H., Kwon, J., Youn, T., Pae, J., Kim, J., Kim, M., et al. (2002). Statistical parametric mapping of LORETA using high density EEG and individual MRI: 
application to mismatch negativity in schizophrenia. Hum. Brain Mapping. 17, 168-178. doi: 10.1002/hbm.10059

Pascual-Marqui, R. (2007). Discrete, 3D distributed, linear imaging methods of electric neuronal activity. Part 1: exact, zero error localization. arXiv:0710.3341 [Preprint].

Pascual-Marqui, R., Lehman, D., Koukkou, M., Kochi, K., Anderer, P., and Saletu, B. (2011). Assessing interactions in the brain with exact low resolution electromagnetic tomography (eLORETA). Philos. Trans. A Math. Phys. Eng. 360, 3768-3784. doi: 10.1098/rsta.2011.008

Pauvermann, M., Lee, G., and Dawson, M. (2017). Glutamatergic regulation of cognition and functional brain connectivity: insights from pharmacological, genetic and translational schizophrenia research. Br. J. Psychiatry 174, 31363160. doi: 10.1111/bph.13919

Pekkonen, E., Katila, H., Ahveninen, J., Karhu, J., Hootilainen, M., and Tiihonen, J. (2002). Impaired temporal lobe processing of preattentive auditory discrimination in schizophrenia. Schizophr. Bull. 28, 467-474. doi: 10.1093/ oxfordjournals.schbul.a006954

Pulvermuller, F., and Shtyrov, Y. (2006). Language outside the focus of attention: the mismatch negativity as a tool for studying higher cognitive processes. Prog. Neurobiol. 79, 49-71. doi: 10.1016/j.pneurobio.2006.04.004

Retsa, C., Knebel, J., Geiser, E., Ferrari, C., Jenni, R., Fournier, M., et al. (2018). Treatment in early psychosis with $\mathrm{N}$-acetyl-cysteine for 6 months improves low-level auditory processing: a pilot study. Schizophr. Res. 191, 80-86. doi: 10.1016/j.schres.2017.07.008

Revheim, N., Corcoran, C., Dias, E., Hellmann, E., Martinez, A., Butler, P., et al. (2014). Reading deficits in schizophrenia and individuals at high clinical risk: relation to sensory function, course of illness, and psychosocial outcome. Am. J. Psychiatry 171, 949-959. doi: 10.1176/appi.ajp.2014.130 91196

Rodionov, V., Durst, R., Mager, M., Teitelbaum, A., and Rashin, S. (2009). Wavelet analysis of the frontal auditory evoked potentials obtained in the passive oddball paradigm in healthy subjects and schizophrenics. J. Basic Clin. Physiol. Pharmacol. 20, 233-264.

Rosburg, T., and Kreitschmann-Andermahr, H. (2016). The effects of ketamine on the mismatch negativity (MMN) in humans - A meta-analysis. Clin. Neurophysiol. 127, 1387-1394. doi: 10.1016/j.clinph.2015.10.062

Saletu, B., Anderer, P., and Saletu-Zyblarz, G. (2006). EEG topography and tomography (LORETA) in the classification and evaluation of the pharmacodynamics of psychotropic drugs. Clin. EEG Neurosci. 37, 66-80. doi: $10.1177 / 155005940603700205$

Salisbury, D., McCathern, A., Coffman, B., Murphy, T., and Haigh, S. (2018) Complex mismatch negativity to tone pair deviants in long-term schizophrenia and in the first-episode schizophrenia spectrum. Schizophr. Res. 191, 18-24. doi: 10.1016/j.schres.2017.04.04

Schall, U., Muller, B., Kargel, C., and Gunturkun, O. (2015). Electrophysiological mismatch response recorded in awake pigeons from the avian functional equivalent of the primary auditory cortex. Neuroreport 26, 239-244. doi: 10 . 1097/WNR.0000000000000323

Schuelert, N., Dorner-Ciosse, C., Brendel, M., and Rosenbrock, H. (2018). comprehensive analysis of auditory event-related potentials and network oscillations in an NMDA receptor antagonist mouse model using a nove wireless recording technology. Physiol. Rep. 6:e13782. doi: 10.14814/phy2.13782

Shtyrov, Y., and Pulvermuller, F. (2007). Language in the mismatch negativity design: motivations, benefits and prospects. J. Psychophysiol. 21, 176-187. doi: 10.1027/0269-8803.21.34.176

Steullet, P., Neijt, H., Cuenod, M., and Do, K. (2006). Synaptic plasticity impairment and hypofunction of NMDA recptors induced by glutathione deficit: relevance to schizophrenia. Neuroscience 137, 807-819. doi: 10.1016/j. neuroscience.2005.10.014

Stone, J., Erlandsson, K., Arstad, E., Squassante, L., Tenneggi, V., Bressan, R., et al. (2008). Relationship between ketamine-induced psychotic symptoms and NMDA receptor occupancy: a [(123)I]CNS-1261 SPET study. Psychopharmacology 197, 401-408. doi: 10.1007/s00213-007-1047-x

Takahashi, H., Rissling, A., Pascual-Marqui, R., Kirihara, K., Pela, M., Sprock, J., et al. (2013). Neural substrates of normal and impaired preattentive sensory discrimination in large cohorts of nonpsychiatric subjects and schizophrenia patients as indexed by MMN and P3a detection responses. Neuroimage 1, 594-603. doi: 10.1016/j.neuroimage.2012.09.074
Tallon-Baudry, C., Bertrand, O., Delpuech, C., and Pernier, J. (1996). Stimulus specificity of phase-locked and non-phase-locked $40 \mathrm{~Hz}$ visual responses in human. J. Neurosci. 16, 4240-4249. doi: 10.1523/jneurosci.16-13-04240.1996

Tamminen, H., Peltola, M., Kujala, T., and Naatanen, R. (2015). Phonetic training and non-native speech perception - How memory traces evolve in just three days as indexed by the mismatch negativity (MMN) and behavioural measures. Int. J. Psychophysiol. 97, 23-29. doi: 10.1016/j.ijpsycho.2015.04.020

Thiebes, S., Leicht, G., Curic, S., Steinmann, S., Polomac, N., Andreou, C. et al. (2017). Glutamatergic deficits and schizophrenia-like negative symptoms: new evidence from ketamine-induced mismatch negativity alternations in healthy male humans. J. Psychiatry Neurosci. 42, 273-283. doi: 10.2174/ 1389201019666180620112528

Thomas, E., Bozaoglu, K., Russell, S., and Gurvich, C. (2017). The influence of the glutamatergic system in cognition in schizophrenia: a systematic review. Neurosci. Biobehav. Rev. 77, 369-397. doi: 10.1016/j.neubiorev.2017.04.005

Thonnesen, H., Zvyagintsev, M., Harke, K., Boers, F., Dammers, J., Norra, C., et al. (2008). Optimized mismatch negativity paradigm reflects deficits in schizophrenia patients. Biol. Psychol. 17, 205-216. doi: 10.1016/j.biopsycho. 2007.10.009

Tikhonravov, D., Neuvonen, T., Pertovaara, A., Savioja, K., Ruusuvirta, T., and Näätänen, R. (2008). Effects of an NMDA-receptor antagonist MK-801 on an MMN-like response recorded in anaesthetized rats. Brain. Res. 1203, 97-102. doi: 10.1016/j.brainres.2008.02.006

Todd, J., Harms, L., Schall, U., and Michi, P. (2013). Mismatch negativity: translating the potential. Front. Psychiatry 4:171. doi: 10.3389/fpsyt.2013. 00171

Todd, J., Michie, P., and Jablensky, A. (2003). Association between reduced duration mismatch negativity (MMN) and raised temporal discrimination thresholds in schizophrenia. Clin. Neurophysiol. 114, 2061-2070. doi: 10.1016/ s1388-2457(03)00246-3

Todd, J., Michie, P., Schall, U., Karayansdis, F., Yabe, H., and Naatanen R. (2008). Deviant matters: duration, frequency, and intensity deviants reveal different patterns of mismatch negativity reduction in early and late schizophrenia. Biol. Psychiatry 63, 58-64. doi: 10.1016/j.biopsych.2007. 02.016

Todd, J., Michie, P., Schall, U., Ward, P., and Catts, S. (2012). Mismatch negativity $(\mathrm{MMN})$ reduction in schizophrenia - Impaired prediction-error generation, estimation or salience. Int. J. Psychophysiol. 83, 222-231. doi: 10.1016/j.ijpsycho. 2011.10 .003

Tramo, M., Shah, G., and Braida, L. (2002). Functional role of auditory cortex in frequency processing and pitch perception. J. Neurophysiol. 87, 122-139. doi: 10.1152/jn.00104.1999

Tse, C., Rinne, T., Ng, K., and Penney, T. (2013). The functional role of the frontal cortex in pre-attentive auditory change detection. Neuroimage 83, 870-879. doi: 10.1016/j.neuroimage.2013.07.037

Umbricht, D., Koller, R., Vollenweider, F., and Schmid, L. (2002). Mismatch negativity predicts psychotic experiences induced by NMDA receptor antagonist in healthy volunteers. Biol. Psychiatry 51, 400-406. doi: 10.1016/ s0006-3223(01)01242-2

Umbricht, D., Vyssotki, D., Latanov, A., Nitsch, R., and Lipp, H. (2005) Deviance-related electrophysiological activity in mice: is there mismatch negativity in mice? Clin. Neurophysiol. 116, 353-363. doi: 10.1016/j.clinph.2004. 08.015

Vollenweider, F., Lienders, K., Scharfetter, C., Antagonini, A., Maguire, P., and Missimer, J. (1987). Metabolic hyperfrontality and psychopathology in the ketamine model of psychosis using positron emission tomography (PET) and [18F] flourodeoxyglucose (FDG). Eur. Neuropsychopharmacology 7, 9-24. doi: 10.1016/s0924-977x(96)00039-9

Wang, Z., Ryan, A., and Woolf, N. (1987). Pentobarbital and ketamine alter the patters of 2-deoxyglucose uptake in the central auditory system of the gerbil. Hear. Res. 27, 145-155. doi: 10.1016/0378-5955(87)90015-3

Wible, C., Kobicki, M., Yoo, S., Kacher, D., Jalisbury, D., Anderson, M., et al (2001). A functional magnetic resonance imaging study of auditory mismatch negativity in schizophrenia. Am. J. Psychiatry 158, 938-943. doi: 10.1176/appi. ajp.158.6.938

Williams, J., Gibbon, M., First, M., Spitzer, R., Davies, M., and Borus, J. (1992). The structured Clinical Interview for the DSM-III-R (SCID). Multisite test-retest reliability. Arch. Gen. Psychiatry 49, 630-636. 
Womelsdorf, T., Valiante, T., Sahin, N., Miller, K., and Tiesinga, P. (2014). Dynamic circuit motifs underlying rhythmic gain control, gating and integration. Nat. Neurosci. 17, 1031-1039. doi: 10.1038/nn.3764

Xiong, Y., Bo, Q., Wang, C., Tian, Q., Liu, Y., and Wang, C. (2019). Differential of frequency and duration mismatch negativity and theta power deficits in firstepisode and chronic schizophrenia. Front. Behav. Neurosci. 6:37. doi: 10.3389/ fnbeh.2019.00037

Yamasue, H., Yamada, H., Yumoto, M., Kamco, S., Kudo, N., Vetsuki, M., et al. (2004). Abnormal association between reduced magnetic mismatch field to speech sounds and smaller planum temporale volume in schizophrenia. Neuroimage 22, 720-727. doi: 10.1016/j.neuroimage.2004. 01.042

Youn, T., Park, H., Kim, J., Kim, M., and Kwon, J. (2003). Altered hemispheric symmetry and positive symptoms in schizophrenia: equivalent current dipole of auditory mismatch negativity. Schizophr. Res. 59, 253-260. doi: 10.1016/s09209964(02)00154-8

Conflict of Interest Statement: The authors declare that the research was conducted in the absence of any commercial or financial relationships that could be construed as a potential conflict of interest.

Copyright (c) 2019 de la Salle, Shah, Choueiry, Bowers, McIntosh, Ilivitsky and Knott. This is an open-access article distributed under the terms of the Creative Commons Attribution License (CC BY). The use, distribution or reproduction in other forums is permitted, provided the original author(s) and the copyright owner(s) are credited and that the original publication in this journal is cited, in accordance with accepted academic practice. No use, distribution or reproduction is permitted which does not comply with these terms. 


\title{
Appendix III: Mini-International Neuropsychiatric Interview (MINI)
}

\section{M.I.N.I.}

\section{MINI INTERNATIONAL NEUROPSYCHIATRIC INTERVIEW}

\author{
English Version 5.0.0
}

DSM-IV

USA: D. Sheehan, J. Janavs, R. Baker, K. Harnett-Sheehan, E. Knapp, M. Sheehan University of South Florida - Tampa

FRANCE: Y. Lecrubier, E. Weiller, T. Hergueta, P. Amorim, L. I. Bonora, J. P. Lépine Hôpital de la Salpétrière - Paris

(C) Copyright 1992-2006 Sheehan DV \& Lecrubier Y

All rights reserved. No part of this document may be reproduced or transmitted in any form, or by any means, electronic or mechanical, including photocopying, or by any information storage or retrieval system, without permission in writing from Dr. Sheehan or Dr. Lecrubier. Researchers and clinicians working in nonprofit or publicly owned settings (including universities, nonprofit hospitals, and government institutions) may make copies of a M.I.N.I. instrument for their own clinical and research use.

\section{DISCLAIMER}

Our aim is to assist in the assessment and tracking of patients with greater efficiency and accuracy. Before action is taken on any data collected and processed by this program, it should be reviewed and interpreted by a licensed clinician.

This program is not designed or intended to be used in the place of a full medical and psychiatric evaluation by a qualified licensed physician - psychiatrist. It is intended only as a tool to facilitate accurate data collection and processing of symptoms elicited by trained personnel.

M.I.N.I. 5.0.0 (July 1, 2006) 


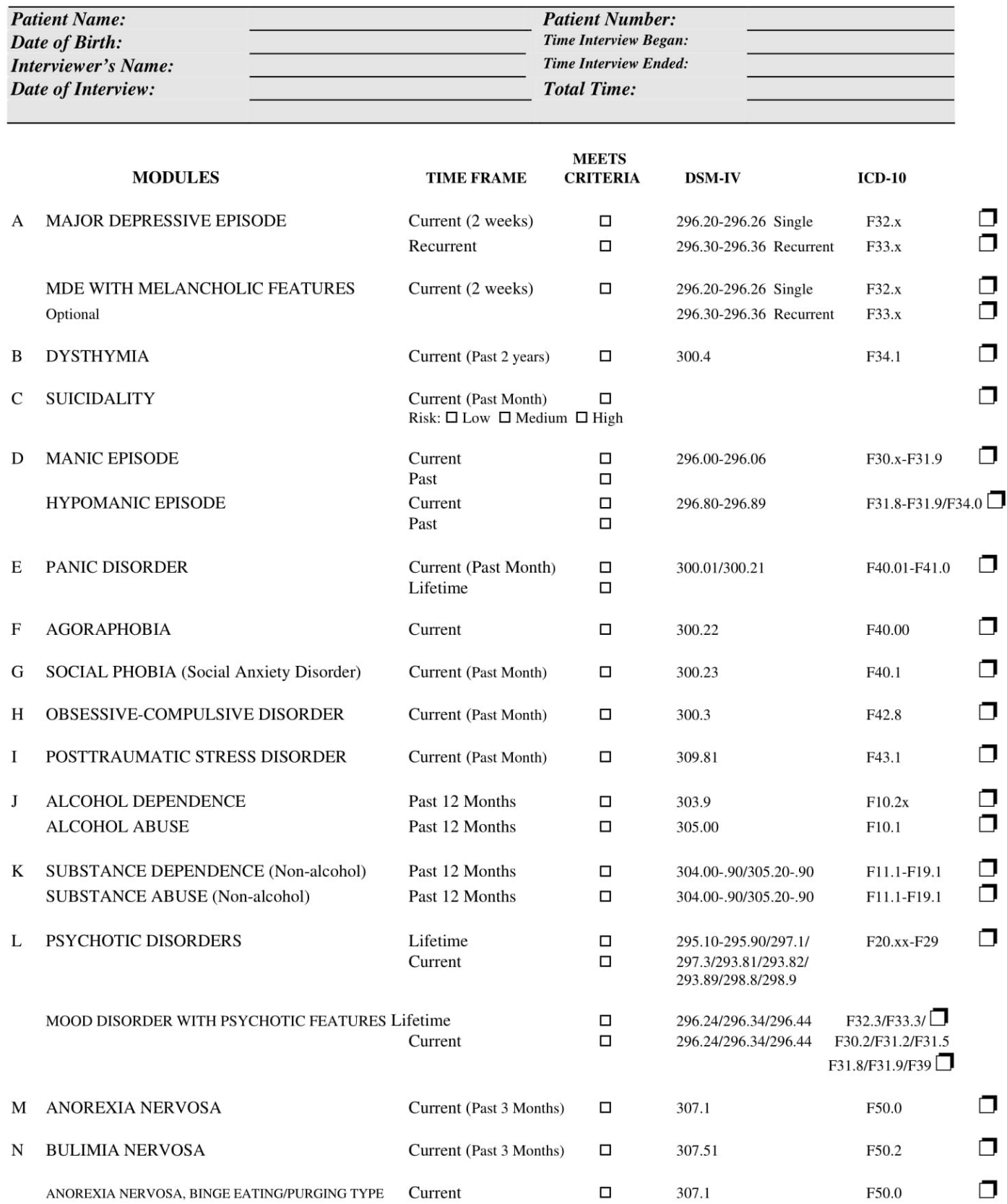


O GENERALIZED ANXIETY DISORDER

Current (Past 6 Months)

$\square$

300.02

F41.1

$\square$

P ANTISOCIAL PERSONALITY DISORDER

Lifetime

$\square$

301.7

F60.2

$\square$

Which problem troubles you the most? Indicate your response by checking the appropriate check box(es). 


\section{GENERAL INSTRUCTIONS}

The M.I.N.I. was designed as a brief structured interview for the major Axis I psychiatric disorders in DSM-IV and ICD-10. Validation and reliability studies have been done comparing the M.I.N.I. to the SCID-P for DSM-III-R and the CIDI (a structured interview developed by the World Health Organization for lay interviewers for ICD-10). The results of these studies show that the M.I.N.I. has acceptably high validation and reliability scores, but can be administered in a much shorter period of time (mean $18.7 \pm$

11.6 minutes, median 15 minutes) than the above referenced instruments. It can be used by clinicians, after a brief training session. Lay interviewers require more extensive training.

\section{INTERVIEW:}

In order to keep the interview as brief as possible, inform the patient that you will conduct a clinical interview that is more structured than usual, with very precise questions about psychological problems which require a yes or no answer.

\section{GENERAL FORMAT:}

The M.I.N.I. is divided into modules identified by letters, each corresponding to a diagnostic category.

-At the beginning of each diagnostic module (except for psychotic disorders module), screening question(s) corresponding to the main criteria of the disorder are presented in a gray box.

-At the end of each module, diagnostic box(es) permit the clinician to indicate whether diagnostic criteria are met.

\section{CONVENTIONS:}

Sentences written in «normal font » should be read exactly as written to the patient in order to standardize the assessment of diagnostic criteria.

Sentences written in « CAPITALS » should not be read to the patient. They are instructions for the interviewer to assist in the scoring of the diagnostic algorithms.

Sentences written in «bold » indicate the time frame being investigated. The interviewer should read them as often as necessary. Only symptoms occurring during the time frame indicated should be considered in scoring the responses.

Answers with an arrow above them $(\Rightarrow)$ indicate that one of the criteria necessary for the diagnosis(es) is not met. In this case, the interviewer should go to the end of the module, circle «NO » in all the diagnostic boxes and move to the next module.

When terms are separated by a slash (/) the interviewer should read only those symptoms known to be present in the patient (for example, question H6).

Phrases in (parentheses) are clinical examples of the symptom. These may be read to the patient to clarify the question.

\section{RATING INSTRUCTIONS:}

All questions must be rated. The rating is done at the right of each question by circling either Yes or No. Clinical judgment by the rater should be used in coding the responses. The rater should ask for examples when necessary, to ensure accurate coding. The patient should be encouraged to ask for clarification on any question that is not absolutely clear.

The clinician should be sure that each dimension of the question is taken into account by the patient (for example, time frame, frequency, severity, and/or alternatives).

Symptoms better accounted for by an organic cause or by the use of alcohol or drugs should not be coded positive in the M.I.N.I. The M.I.N.I. Plus has questions that investigate these issues.

For any questions, suggestions, need for a training session, or information about updates of the M.I.N.I., please contact :

\author{
David V Sheehan, M.D., M.B.A. \\ University of South Florida College of Medicine \\ 3515 East Fletcher Avenue \\ Tampa, FL USA 33613-4788 \\ tel : +1 813974 4544; fax : +1 8139744575 \\ e-mail :dsheehan@hsc.usf.edu
}

M.I.N.I. 5.0.0 (July 1, 2006)
Yves Lecrubier, M.D. / Thierry Hergueta, M.S. INSERM U302

Hôpital de la Salpétrière

47, boulevard de l'Hôpital

F. 75651 PARIS, FRANCE

tel : +33(0) 142161659 ; fax : +33 (0) 145852800

e-mail : hergueta@ext.jussieu.fr 


\section{A. MAJOR DEPRESSIVE EPISODE}

$\Rightarrow$ MEANS : GO TO THE DIAGNOSTIC BOXES, CIRCLE NO IN ALL DIAGNOSTIC BOXES, AND MOVE TO THE NEXT MODULE)

\begin{tabular}{llc}
\hline A1 & $\begin{array}{l}\text { Have you been consistently depressed or down, most of the day, nearly } \\
\text { every day, for the past two weeks? }\end{array}$ & NO \\
A2 $\begin{array}{l}\text { In the past two weeks, have you been much less interested in most things or } \\
\text { much less able to enjoy the things you used to enjoy most of the time? }\end{array}$ & NO & YES \\
& IS A1 OR A2 CODED YES? & NO \\
\hline
\end{tabular}

A3 Over the past two weeks, when you felt depressed or uninterested:

a Was your appetite decreased or increased nearly every day? Did your weigh decrease or increase without trying intentionally (i.e., by $\pm 5 \%$ of body weight or $\pm 8 \mathrm{lbs}$. or $\pm 3.5 \mathrm{kgs}$., for a $160 \mathrm{lb} . / 70 \mathrm{~kg}$. person in a month)? IF YES TO EITHER, CODE YES.

b Did you have trouble sleeping nearly every night (difficulty falling asleep, waking up NO YES in the middle of the night, early morning wakening or sleeping excessively)?

c Did you talk or move more slowly than normal or were you fidgety, restless or having trouble sitting still almost every day?

d Did you feel tired or without energy almost every day?

NO YES *

e Did you feel worthless or guilty almost every day?

f Did you have difficulty concentrating or making decisions almost every day?

NO YES *

g Did you repeatedly consider hurting yourself, feel suicidal, or wish that you were dead?

NO YES

NO YES

NO YES

NO YES

ARE 5 OR MORE ANSWERS (A1-A3) CODED YES?

NO

YES *

MAJOR DEPRESSIVE

EPISODE, CURRENT

IF PATIENT HAS CURRENT MAJOR DEPRESSIVE EPISODE CONTINUE TO A4, OTHERWISE MOVE TO MODULE B:

A4 a During your lifetime, did you have other episodes of two weeks or more when you felt depressed or uninterested in most things, and had most of the problems we just talked about?

b In between 2 episodes of depression, did you ever have an interval

NO

YES of at least 2 months, without any depression and any loss of interest?

* If patient has Major Depressive Episode, Current, use this information in coding the corresponding questions on page 5 (A6d, A6e). 


\section{MAJOR DEPRESSIVE EPISODE WITH MELANCHOLIC FEATURES (optional)}

$(\Rightarrow$ MEANS : GO TO THE DIAGNOSTIC BOX, CIRCLE NO, AND MOVE TO THE NEXT MODULE)

IF THE PATIENT CODES POSITIVE FOR A CURRENT MAJOR DEPRESSIVE EPISODE (A3 = YES), EXPLORE THE FOLLOWING:

A5 a During the most severe period of the current depressive episode, did you lose almost

NO YES completely your ability to enjoy nearly everything?

b During the most severe period of the current depressive episode, did you lose your ability to respond to things that previously gave you pleasure, or cheered you up?

IF NO: When something good happens does it fail to make you feel better, even temporarily?

IS EITHER A5a OR A5b CODED YES?

NO YES

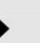

NO YES

A6 Over the past two week period, when you felt depressed and uninterested:

a Did you feel depressed in a way that is different from the kind of feeling you experience when someone close to you dies?

b Did you feel regularly worse in the morning, almost every day?

c Did you wake up at least 2 hours before the usual time of awakening and have difficulty getting back to sleep, almost every day?

d IS A3c CODED YES (PSYCHOMOTOR RETARDATION OR AGITATION)?

e IS A3a CODED YES FOR ANOREXIA OR WEIGHT LOSS?

f Did you feel excessive guilt or guilt out of proportion to the reality of the situation?

ARE 3 OR MORE A6 ANSWERS CODED YES?

NO

YES

Major Depressive Episode with

Melancholic Features

Current 


\section{B. DYSTHYMIA}

$(\Rightarrow$ MEANS : GO TO THE DIAGNOSTIC BOX, CIRCLE NO, AND MOVE TO THE NEXT MODULE)

IF PATIENT'S SYMPTOMS CURRENTLY MEET CRITERIA FOR MAJOR DEPRESSIVE EPISODE, DO NOT EXPLORE THIS MODULE.

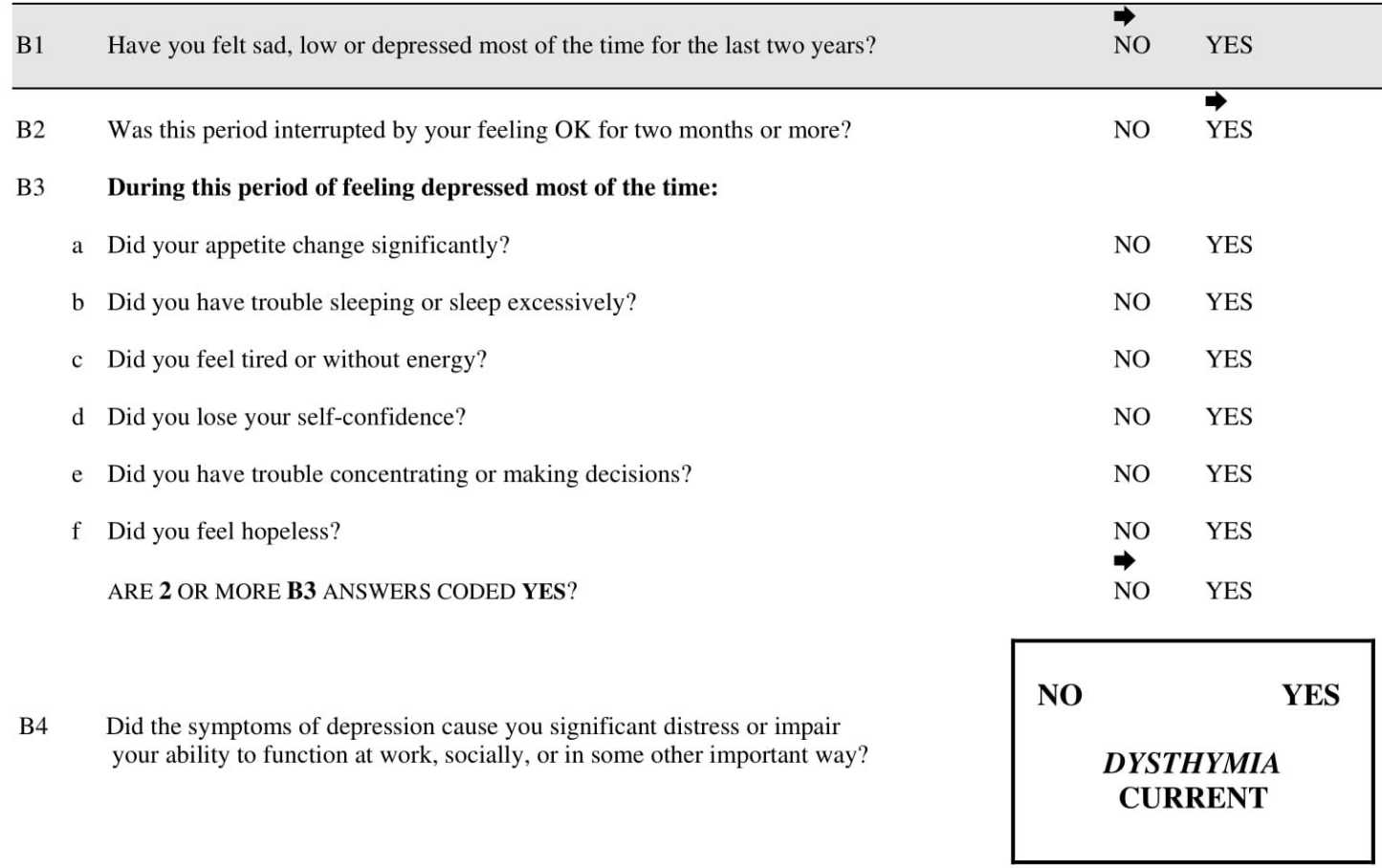




\section{SUICIDALITY}

In the past month did you:

C1 Suffer any accident?

IF NO TO C1, SKIP TO C2; IF YES, ASK C1a,

$\mathrm{C} 1 \mathrm{a} \quad \mathrm{Plan}$ or intend to hurt yourself in that accident either passively or actively?

IF NO TO C1a, SKIP TO C2: IF YES, ASK C1b,:

$\mathrm{C} 1 \mathrm{~b}$ Did you intend to die as a result of this accident?

C2 Think that you would be better off dead or wish you were dead?

C3 Want to harm yourself or to hurt or to injure yourself?

C4 Think about suicide?

$\begin{array}{ccc}\text { NO } & \text { YES } & \text { Points } \\ \text { NO } & \text { YES } & 0 \\ \text { NO } & \text { YES } & 0 \\ \text { NO } & \text { YES } & 1 \\ \text { NO } & \text { YES } & 2 \\ \text { NO } & \text { YES } & 6\end{array}$

IF YES, ASK ABOUT THE INTENSITY AND FREQUENCY OF THE SUICIDAL IDEATION:

Frequency Intensity

\begin{tabular}{|lll|l} 
Occasionally & $\square$ & Mild & $\square$ \\
Often & $\square$ & $\begin{array}{l}\text { Moderate } \\
\text { Serere }\end{array}$ & $\square$
\end{tabular}$\quad \begin{aligned} & \text { Can you control these impulses } \\
& \text { and state that you will not act } \\
& \text { on them while in this program? }\end{aligned}$

Only score 8 points if response is NO. NO YES 8

C5

Have a suicide plan?

NO YES

C6 Take any active steps to prepare to injure yourself or to prepare for a suicide attempt in which you expected or intended to die?

C7 Deliberately injure yourself without intending to kill yourself?

C8 Attempt suicide?

Hoped to be rescued / survive

Expected / intended to die

In your lifetime:

C9 Did you ever make a suicide attempt?

NO YES 4

IS AT LEAST 1 OF THE ABOVE (EXCEPT C1) CODED YES?

IF YES, ADD THE TOTAL NUMBER OF POINTS FOR THE ANSWERS (C1-C9) CHECKED 'YES' AND SPECIFY THE LEVEL OF SUICIDE RISK AS INDICATED IN THE DIAGNOSTIC BOX:

MAKE ANY ADDITIONAL COMMENTS ABOUT YOUR ASSESSMENT OF THIS PATIENT'S CURRENT AND NEAR FUTURE SUICIDE RISK IN THE SPACE BELOW:

NO YES

SUICIDE RISK CURRENT

1-8 points Low 9-16 points Moderate

$\geq 17$ points High 


\section{D. (HYPO) MANIC EPISODE}

$\rightarrow$ MEANS : GO TO THE DIAGNOSTIC BOXES, CIRCLE NO IN ALL DIAGNOSTIC BOXES, AND MOVE TO THE NEXT MODULE)

D1 a Have you ever had a period of time when you were feeling 'up' or 'high' or 'hyper'

NO YES or so full of energy or full of yourself that you got into trouble, or that other people thought you were not your usual self? (Do not consider times when you were intoxicated on drugs or alcohol.)

IF PATIENT IS PUZZLED OR UNCLEAR ABOUT WHAT YOU MEAN BY 'UP' OR 'HIGH' OR 'HYPER', CLARIFY AS FOLLOWS: By 'up' or 'high' or 'hyper' I mean: having elated mood; increased energy; needing less sleep; having rapid thoughts; being full of ideas; having an increase in productivity, motivation, creativity, or impulsive behavior.

IF NO, CODE NO TO D1b: IF YES ASK:

b Are you currently feeling 'up' or 'high' or 'hyper' or full of energy?

D2 a Have you ever been persistently irritable, for several days, so that you had arguments or verbal or physical fights, or shouted at people outside your family? Have you or others noticed that you have been more irritable or over reacted, compared to other people, even in situations that you felt were justified?

IF NO, CODE NO TO D2b: IF YES ASK:

b Are you currently feeling persistently irritable?

IS D1a OR D2a CODED YES?

D3 IF D1b OR D2b = YES: EXPLORE THE CURRENT AND THE MOST SYMPTOMATIC PAST EPISODE, OTHERWISE IF D1b AND D2b = NO: EXPLORE ONLY THE MOST SYMPTOMATIC PAST EPISODE

During the times when you felt high, full of energy, or irritable did you:

a Feel that you could do things others couldn't do, or that you were an especially important person? IF YES, ASK FOR EXAMPLES.

THE EXAMPLES ARE CONSISTENT WITH A DELUSIONAL IDEA. $\square$ No $\square$ Yes

b Need less sleep (for example, feel rested after only a few hours sleep)?

c Talk too much without stopping, or so fast that people had difficulty understanding?

d Have racing thoughts?

\begin{tabular}{lc|cc}
\multicolumn{2}{c|}{ Current Episode } & \multicolumn{2}{c}{ Past Episode } \\
NO & YES & NO & YES \\
& & & \\
NO & YES & NO & YES \\
NO & YES & NO & YES \\
NO & YES & NO & YES \\
NO & YES & NO & YES \\
NO & YES & NO & YES \\
NO & YES & NO & YES
\end{tabular}

g Want so much to engage in pleasurable activities that you ignored the risks or consequences (for example, spending sprees, reckless driving, or sexual indiscretions)? 


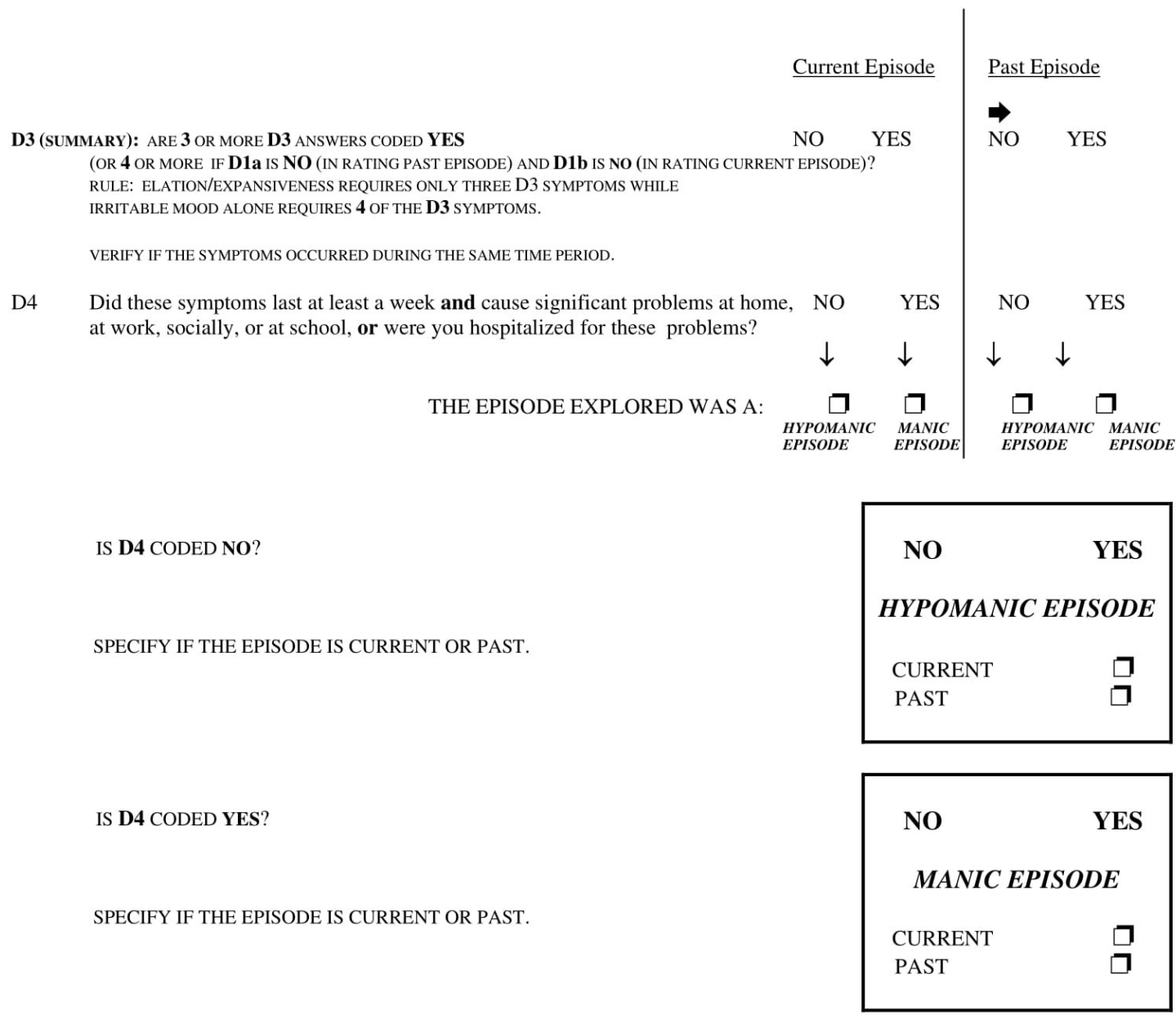




\section{E. PANIC DISORDER}

$\Leftrightarrow$ MEANS : CirCle NO IN E5, E6 AND E7 AND SKIP To F1)

E1 a Have you, on more than one occasion, had spells or attacks when you suddenly felt anxious, frightened, uncomfortable or uneasy, even in situations where most people would not feel that way?

b Did the spells surge to a peak within 10 minutes of starting? or occur in an unpredictable or unprovoked manner?

E3 Have you ever had one such attack followed by a month or more of persistent concern about having another attack, or worries about the consequences of the attack or did you make a significant change in your behavior because of the attacks (e.g., shopping only with a companion, not wanting to leave your house, visiting the emergency room repeatedly, or seeing your doctor more frequently because of the symptoms?

E4 During the worst spell that you can remember:

a Did you have skipping, racing or pounding of your heart?

NO YES

b Did you have sweating or clammy hands?

NO YES

c Were you trembling or shaking?

d Did you have shortness of breath or difficulty breathing?

NO YES

e Did you have a choking sensation or a lump in your throat?

NO YES

f Did you have chest pain, pressure or discomfort?

NO YES

g Did you have nausea, stomach problems or sudden diarrhea?

NO YES

h Did you feel dizzy, unsteady, lightheaded or faint?

NO YES

i Did things around you feel strange, unreal, detached or unfamiliar, or did you feel outside of or detached from part or all of your body?

j Did you fear that you were losing control or going crazy?

NO YES

NO YES

NO YES

$\mathrm{k}$ Did you fear that you were dying?

NO YES

1 Did you have tingling or numbness in parts of your body?

NO YES

m Did you have hot flushes or chills?

E5

ARE BOTH E3, AND 4 OR MORE E4 ANSWERS, CODED YES?

IF YES TO E5, SKIP TO E7.

E6

IF E5 = NO, ARE ANY E4 ANSWERS CODED YES?

THEN SKIP TO F1.

E7 In the past month, did you have such attacks repeatedly ( 2 or more) followed by persistent concern about having another attack?

M.I.N.I. 5.0.0 (July 1, 2006)
NO YES

NO YES

PANIC DISORDER

PANIC DISO
LIFETIME

NO YES

YES LIMITED SYMPTOM
ATTACKS LIFETIME

NO YES

PANIC DISORDER CURRENT 


\section{F. AGORAPHOBIA}

F1 Do you feel anxious or uneasy in places or situations where you might have a panic attack or the panic-like symptoms we just spoke about, or where help might not be available or

escape might be difficult: like being in a crowd, standing in a line (queue),

when you are alone away from home or alone at home, or when crossing a bridge,

traveling in a bus, train or car?

IF F1 = NO, CIRCLE NO IN F2

F2 Do you fear these situations so much that you avoid them, or suffer through them, or need a companion to face them?

NO YES AGORAPHOBIA CURRENT

IS F2 (CURRENT AGORAPHOBIA) CODED NO

and

IS E7 (CURRENT PANIC DISORDER) CODED YES?

NO

YES

PANIC DISORDER

without Agoraphobia CURRENT

IS F2 (CURRENT AGORAPHOBIA) CODED YES

and

IS E7 (CURRENT PANIC DISORDER) CODED YES?

NO

YES

PANIC DISORDER

with Agoraphobia

CURRENT

IS F2 (CURRENT AGORAPHOBIA) CODED YES

and

IS E5 (PANIC DISORDER LIFETIME) CODED NO?

NO

YES

AGORAPHOBIA, CURRENT without history of Panic Disorder 


\section{G. SOCIAL PHOBIA (Social Anxiety Disorder)}

$(\Rightarrow$ MEANS : GO TO THE DIAGNOSTIC BOX, CIRCLE NO AND MOVE TO THE NEXT MODULE)

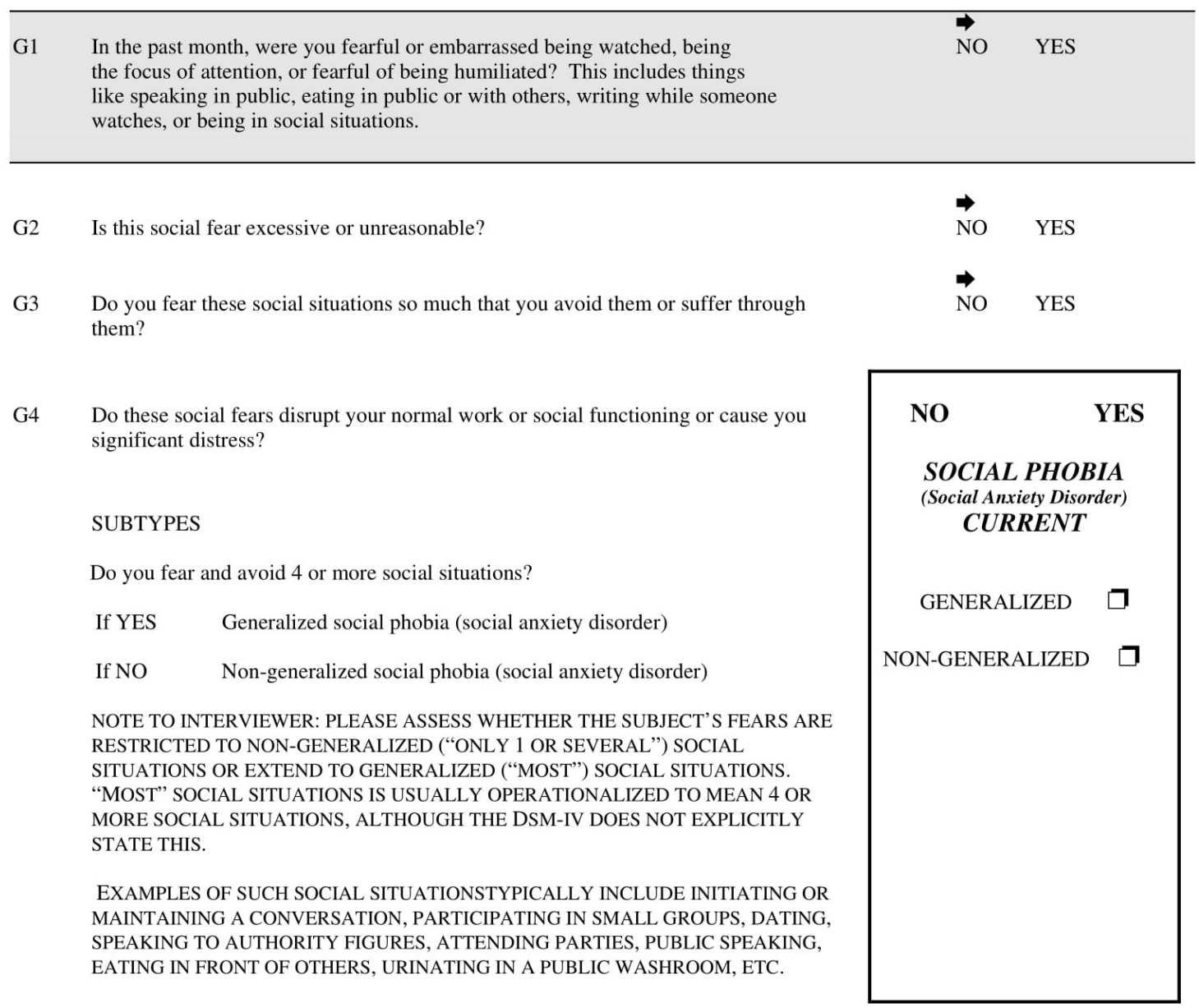




\section{H. OBSESSIVE-COMPULSIVE DISORDER}

$\Rightarrow$ MEANS: GO TO THE DIAGNOSTIC BOX, CIRCLE NO AND MOVE TO THE NEXT MODULE)

H1

the past month, have you been bothered by recurrent thoughts, impulses, or

images that were unwanted, distasteful, inappropriate, intrusive, or distressing?

(For example, the idea that you were dirty, contaminated or had germs, or fear of

contaminating others, or fear of harming someone even though you didn't want to,

or fearing you would act on some impulse, or fear or superstitions that you would

be responsible for things going wrong, or obsessions with sexual thoughts, images

or impulses, or hoarding, collecting, or religious obsessions.)

(DO NOT INCLUDE SIMPLY EXCESSIVE WORRIES ABOUT REAL LIFE PROBLEMS. DO NOT INCLUDE OBSESSIONS DIRECTLY RELATED TO EATING DISORDERS, SEXUAL DEVIATIONS, PATHOLOGICAL GAMBLING, OR ALCOHOL OR DRUG ABUSE BECAUSE THE PATIENT MAY DERIVE PLEASURE FROM THE ACTIVITY AND MAY WANT TO RESIST IT ONLY BECAUSE OF ITS NEGATIVE CONSEQUENCES.)

H2 Did they keep coming back into your mind even when you tried to ignore or get rid of them?

H3 Do you think that these obsessions are the product of your own mind and that they are not imposed from the outside?
NO YES

$\downarrow$

SKIP TO H4

44 In the past month, did you do something repeatedly without being able to resist doing it, like washing or cleaning excessively, counting or checking things over and over, or repeating, collecting, arranging things, or other superstitious rituals?

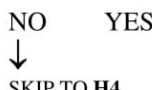

SKIP TO H4

NO YES obsessions
IS H3 OR H4 CODED YES?

H5

Did you recognize that either these obsessive thoughts or these compulsive behaviors were excessive or unreasonable?

H6

Did these obsessive thoughts and/or compulsive behaviors significantly interfere with your normal routine, your work or school, your usual socia activities, or relationships, or did they take more than one hour a day?
NO

O.C.D.

CURRENT 


\section{POSTTRAUMATIC STRESS DISORDER (optional)}

$\Rightarrow$ MEANS : GO TO THE DIAGNOSTIC BOX, CIRCLE NO, AND MOVE TO THE NEXT MODULE)

I1

ave you ever experienced or witnessed or had to deal with an extremely traumatic event that included actual or threatened death or serious injury to you or someone else?

EXAMPLES OF TRAUMATIC EVENTS INCLUDE: SERIOUS ACCIDENTS, SEXUAL OR PHYSICAL ASSAULT, A TERRORIST ATTACK, BEING HELD HOSTAGE, KIDNAPPING, FIRE, DISCOVERING A BODY, SUDDEN DEATH OF SOMEONE CLOSE TO YOU, WAR, OR NATURAL DISASTER.

I2

Did you respond with intense fear, helplessness or horror?

During the past month, have you re-experienced the event in a distressing way (such as, dreams, intense recollections, flashbacks or physical reactions)?
NO YES

NO YES

NO YES

I4

In the past month:

a Have you avoided thinking about or talking about the event ?

NO YES

b Have you avoided activities, places or people that remind you of the event?

NO YES

c Have you had trouble recalling some important part of what happened?

NO YES

d Have you become much less interested in hobbies or social activities?

NO YES

e Have you felt detached or estranged from others?

NO YES

f Have you noticed that your feelings are numbed?

NO YES

g Have you felt that your life will be shortened or that you will die sooner than other people? ARE 3 OR MORE I4 ANSWERS CODED YES?

NO YES

NO YES

I5 In the past month:

a Have you had difficulty sleeping?

NO YES

b Were you especially irritable or did you have outbursts of anger?

NO YES

c Have you had difficulty concentrating?

NO YES

d Were you nervous or constantly on your guard?

NO YES

e Were you easily startled?

ARE 2 OR MORE I5 ANSWERS CODED YES?

NO YES

$\overrightarrow{N O}$ YES

NO

YES

I6 During the past month, have these problems significantly interfered with your work or social activities, or caused significant distress?

POSTTRAUMATIC

STRESS DISORDER

CURRENT 


\section{J. ALCOHOL ABUSE AND DEPENDENCE}

( $\Rightarrow$ MEANS: GO TO DIAGNOSTIC BOXES, CIRCLE NO IN BOTH AND MOVE TO THE NEXT MODULE)

J1 In the past $\mathbf{1 2}$ months, have you had 3 or more alcoholic drinks within a

NO YES 3 hour period on 3 or more occasions?

$\mathrm{J} 2$

In the past 12 months:

a Did you need to drink more in order to get the same effect that you got when you first started drinking?

NO YES

When you cut down on drinking did your hands shake, did you sweat or feel agitated? Did you drink to avoid these symptoms or to avoid being hungover, for example, "the shakes", sweating or agitation?

IF YES TO EITHER, CODE YES

c During the times when you drank alcohol, did you end up drinking more than you planned when you started?

d Have you tried to reduce or stop drinking alcohol but failed?

NO YES

On the days that you drank, did you spend substantial time in obtaining alcohol, drinking, or in recovering from the effects of alcohol?

f Did you spend less time working, enjoying hobbies, or being with others because of your drinking?

g Have you continued to drink even though you knew that the drinking caused you health or mental problems?

ARE 3 OR MORE $\mathbf{J} 2$ ANSWERS CODED YES?

* IF YES, SKIP J3 QUESTIONS, CIRCLE N/A IN THE ABUSE BOX AND MOVE TO THE NEXT DISORDER. DEPENDENCE PREEMPTS ABUSE.

In the past 12 months:

a Have you been intoxicated, high, or hungover more than once when you had other responsibilities at school, at work, or at home? Did this cause any problems? (CODE YES ONLY IF THIS CAUSED PROBLEMS.)

b Were you intoxicated more than once in any situation where you were physically at risk, for example, driving a car, riding a motorbike, using machinery, boating, etc.?

c Did you have legal problems more than once because of your drinking, for example, an arrest or disorderly conduct?

d Did you continue to drink even though your drinking caused problems with your family or other people?

ARE 1 OR MORE J3 ANSWERS CODED YES?

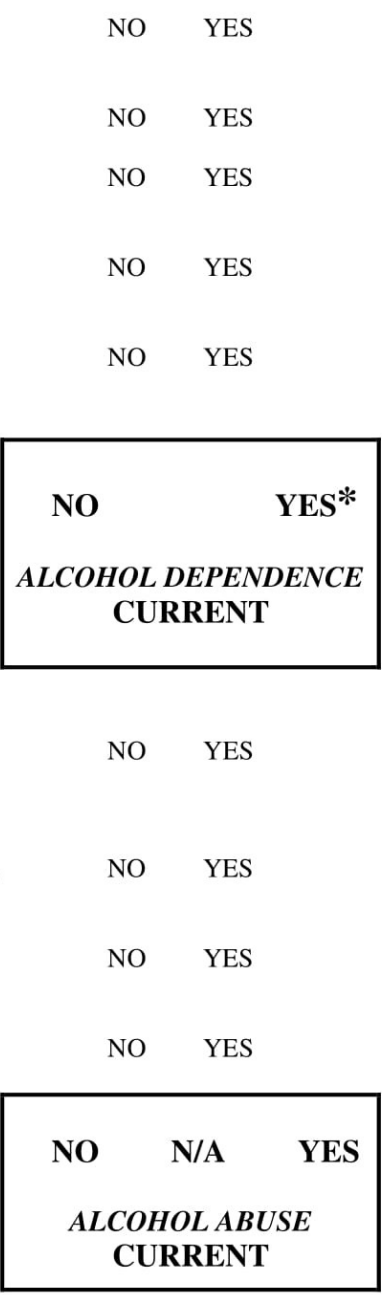




\section{K. NON-ALCOHOL PSYCHOACTIVE SUBSTANCE USE DISORDERS}

$\Rightarrow$ MEANS : GO TO THE DIAGNOSTIC BOXES, CIRCLE NO IN ALL DIAGNOSTIC BOXES, AND MOVE TO THE NEXT MODULE)

Now I am going to show you / read to you a list of street drugs or medicines.

K1 a In the past 12 months, did you take any of these drugs more than once, to get high, to feel better, or to change your mood?

CIRCLE EACH DRUG TAKEN:

Stimulants: amphetamines, "speed", crystal meth, "crank", "rush", Dexedrine, Ritalin, diet pills.

Cocaine: snorting, IV, freebase, crack, "speedball".

Narcotics: heroin, morphine, Dilaudid, opium, Demerol, methadone, codeine, Percodan, Darvon, OxyContin.

Hallucinogens: LSD ("acid"), mescaline, peyote, PCP ("angel dust", "peace pill"), psilocybin, STP, "mushrooms", “ecstasy”, MDA, MDMA, or ketamine ("special K”).

Inhalants: "glue", ethyl chloride, "rush", nitrous oxide ("laughing gas"), amyl or butyl nitrate ("poppers").

Marijuana: hashish ("hash"), THC, "pot", "grass", "weed", "reefer".

Tranquilizers: Quaalude, Seconal ("reds"), Valium, Xanax, Librium, Ativan, Dalmane, Halcion, barbiturates, Miltown, GHB, Roofinol, "Roofies".

Miscellaneous: steroids, nonprescription sleep or diet pills. Any others?

SPECIFY MOST USED DRUG(S):

ONLY ONE DRUG / DRUG CLASS HAS BEEN USED

ONLY THE MOST USED DRUG CLASS IS INVESTIGATED.

EACH DRUG CLASS USED IS EXAMINED SEPARATELY (PHOTOCOPY K2 AND K3 AS NEEDED)

b SPECIFY WHICH DRUG/DRUG CLASS WILL BE EXPLORED IN THE INTERVIEW BELOW IF THERE IS CONCURRENT OR SEQUENTIAL POLYSUBSTANCE USE:

K2 Considering your use of (NAME THE DRUG / DRUG CLASS SELECTED), in the past 12 months:

a Have you found that you needed to use more (NAME OF DRUG / DRUG CLASS SELECTED) to get the same effect that you did when you first started taking it?

b When you reduced or stopped using (NAME OF DRUG / DRUG CLASS SELECTED), did you have withdrawal symptoms (aches, shaking, fever, weakness, diarrhea, nausea, sweating, heart pounding, difficulty sleeping, or feeling agitated, anxious, irritable, or depressed)? Did you use any drug(s) to keep yourself from getting sick (withdrawal symptoms) or so that you would feel better?

IF YES TO EITHER, CODE YES

c Have you often found that when you used (NAME OF DRUG / DRUG CLASS SELECTED), you ended up taking more than you thought you would?

d Have you tried to reduce or stop taking (NAME OF DRUG / DRUG CLASS SELECTED) but failed?

CHECK ONE BOX

On the days that you used (NAME OF DRUG / DRUG CLASS SELECTED), did you spend substantial time ( $>2$ HOURS), obtaining, using or in recovering from the drug, or thinking about the drug?

NO YES

NO YES

YES

NO YES

NO YES

NO YES 
f Did you spend less time working, enjoying hobbies, or being with family or friends because of your drug use?

g Have you continued to use (NAME OF DRUG / DRUG CLASS SELECTED), even though it caused you health or mental problems?

ARE 3 OR MORE K2 ANSWERS CODED YES?

SPECIFY DRUG(S):

* IF YES, SKIP K3 QUESTIONS, CIRCLE N/A IN THE ABUSE BOX FOR THIS SUBSTANCE AND MOVE TO THE NEXT DISORDER. DEPENDENCE PREEMPTS ABUSE.

\begin{tabular}{|c|}
\hline NO YES * \\
SUBSTANCE DEPENDENCE \\
CURRENT \\
\end{tabular}

Considering your use of (NAME THE DRUG CLASS SELECTED), in the past 12 months:

K3 a Have you been intoxicated, high, or hungover from (NAME OF DRUG / DRUG CLASS SELECTED) more than once, when you had other responsibilities at school, at work, or at home? Did this cause any problem?

(CODE YES ONLY IF THIS CAUSED PROBLEMS.)

b Have you been high or intoxicated from (NAME OF DRUG / DRUG CLASS SELECTED) more than once in any situation where you were physically at risk (for example, driving a car, riding a motorbike, using machinery, boating, etc.)?

c Did you have legal problems more than once because of your drug use, for example, an arrest or disorderly conduct?

d Did you continue to use (NAME OF DRUG / DRUG CLASS SELECTED), even though it caused problems with your family or other people?

ARE 1 OR MORE K3 ANSWERS CODED YES?

SPECIFY DRUG(S): 


\section{PSYCHOTIC DISORDERS AND MOOD DISORDER WITH PSYCHOTIC FEATURES}

ASK FOR AN EXAMPLE OF EACH QUESTION ANSWERED POSITIVELY. CODE YES ONLY IF THE EXAMPLES CLEARLY SHOW A DISTORTION OF THOUGHT OR OF PERCEPTION OR IF THEY ARE NOT CULTURALLY APPROPRIATE. BEFORE CODING, INVESTIGATE WHETHER DELUSIONS QUALIFY AS "BIZARRE".

DELUSIONS ARE "BIZARRE" IF: CLEARLY IMPLAUSIBLE, ABSURD, NOT UNDERSTANDABLE, AND CANNOT DERIVE FROM ORDINARY LIFE EXPERIENCE.

HALLUCINATIONS ARE SCORED "BIZARRE" IF: A VOICE COMMENTS ON THE PERSON'S THOUGHTS OR BEHAVIOR, OR WHEN TWO OR MORE VOICES ARE CONVERSING WITH EACH OTHER.

Now I am going to ask you about unusual experiences that some people have.

BIZARRE

L1 a Have you ever believed that people were spying on you, or that someone was plotting against you, or trying to hurt you? NOTE: ASK FOR EXAMPLES TO RULE OUT ACTUAL STALKING.

b IF YES OR YES BIZARRE: do you currently believe these things?

\begin{tabular}{|c|c|c|}
\hline & & BIZARRE \\
\hline NO & YES & YES \\
\hline NO & YES & $\begin{array}{l}\text { YES } \\
\Rightarrow \text { L6 }\end{array}$ \\
\hline NO & YES & YES \\
\hline NO & YES & $\begin{array}{l}\text { YES } \\
\Rightarrow \text { L6 }\end{array}$ \\
\hline NO & YES & YES \\
\hline $\mathrm{NO}$ & YES & $\begin{array}{l}\text { YES } \\
\Rightarrow \text { L6 }\end{array}$ \\
\hline $\mathrm{NO}$ & YES & YES \\
\hline NO & YES & $\begin{array}{l}\text { YES } \\
\Rightarrow \text { L6 }\end{array}$ \\
\hline NO & YES & YES \\
\hline NO & YES & YES \\
\hline $\mathrm{NO}$ & YES & \\
\hline NO & & YES \\
\hline $\mathrm{NO}$ & YES & $\begin{array}{l}\text { YES } \\
\Rightarrow L \mathbf{L b}\end{array}$ \\
\hline
\end{tabular}

b IF YES OR YES BIZARRE TO L6a: have you heard these things in the past month? HALLUCINATIONS ARE SCORED "BIZARRE" ONLY IF PATIENT ANSWERS YES TO THE FOLLOWING: Did you hear a voice commenting on your thoughts or behavior or did you hear two or more voices talking to each other? 
L7 a Have you ever had visions when you were awake or have you ever seen things

CLINICIAN: CHECK TO SEE IF THESE ARE CULTURALLY INAPPROPRIATE.

b IF YES: have you seen these things in the past month?

CLINICIAN'S JUDGMENT

L8 b IS THE PATIENT CURRENTLY EXHIBITING INCOHERENCE, DISORGANIZED

L9 b IS THE PATIENT CURRENTLY EXHIBITING DISORGANIZED OR CATATONIC BEHAVIOR?

L10 b ARE NEGATIVE SYMPTOMS OF SCHIZOPHRENIA, E.G. SIGNIFICANT AFFECTIVE FLATTENING, POVERTY OF SPEECH (ALOGIA) OR AN INABILITY TO INITIATE OR PERSIST IN GOAL-DIRECTED ACTIVITIES (AVOLITION), PROMINENT DURING THE INTERVIEW?

L11 a ARE 1 OR MORE « a » QUESTIONS FROM L1a TO L7a CODED YES OR YES BIZARRE AND IS EITHER:

MAJOR DEPRESSIVE EPISODE, (CURRENT OR RECURRENT)

OR

MANIC OR HYPOMANIC EPISODE, (CURRENT OR PAST) CODED YES?

IF NO TO L11 a, CIRCLE NO IN BOTH 'MOOD DISORDER WITH PSYCHOTIC FEATURES’ DIAGNOSTIC BOXES AND MOVE TO L13.

b You told me earlier that you had period(s) when you felt (depressed/high/persistently irritable).

NO YES

Were the beliefs and experiences you just described (SYMPTOMS CODED YES FROM L1a TO L7a) restricted exclusively to times when you were feeling depressed/high/irritable?

IF THE PATIENT EVER HAD A PERIOD OF AT LEAST 2 WEEKS OF HAVING THESE BELIEFS OR EXPERIENCES (PSYCHOTIC SYMPTOMS) WHEN THEY WERE NOT DEPRESSED/HIGH/IRRITABLE, CODE NO TO THIS DISORDER. AND IS EITHER:

MAJOR DEPRESSIVE EPISODE, (CURRENT)

OR

MANIC OR HYPOMANIC EPISODE, (CURRENT) CODED YES?

MOOD DISORDER WITH

PSYCHOTIC FEATURES

CURRENT

IF THE ANSWER IS YES TO THIS DISORDER (LIFETIME OR CURRENT),

CIRCLE NO TO L13 AND L14 AND MOVE TO THE NEXT MODULE. 
L13 ARE 1 OR MORE « $\mathrm{b}$ » QUESTIONS FROM L1b TO L6b, CODED YES BIZARRE? OR

ARE 2 OR MORE « $\mathrm{b} »$ QUESTIONS FROM L1b TO L10b, CODED YES (RATHER THAN YES BIZARRE)?

AND DID AT LEAST TWO OF THE PSYCHOTIC SYMPTOMS OCCUR DURING THE SAME 1 MONTH PERIOD?

L14 IS L13 CODED YES

OR

ARE 1 OR MORE « a » QUESTIONS FROM L1a TO L6a, CODED YES BIZARRE?

OR

NO YES
PSYCHOTIC DISORDER
CURRENT

\begin{tabular}{|c|}
\hline NO YES \\
PSYCHOTIC DISORDER \\
LIFETIME \\
\end{tabular}




\section{ANOREXIA NERVOSA}

$\Rightarrow$ MEANS : GO TO THE DIAGNOSTIC BOX, CIRCLE NO, AND MOVE TO THE NEXT MODULE)

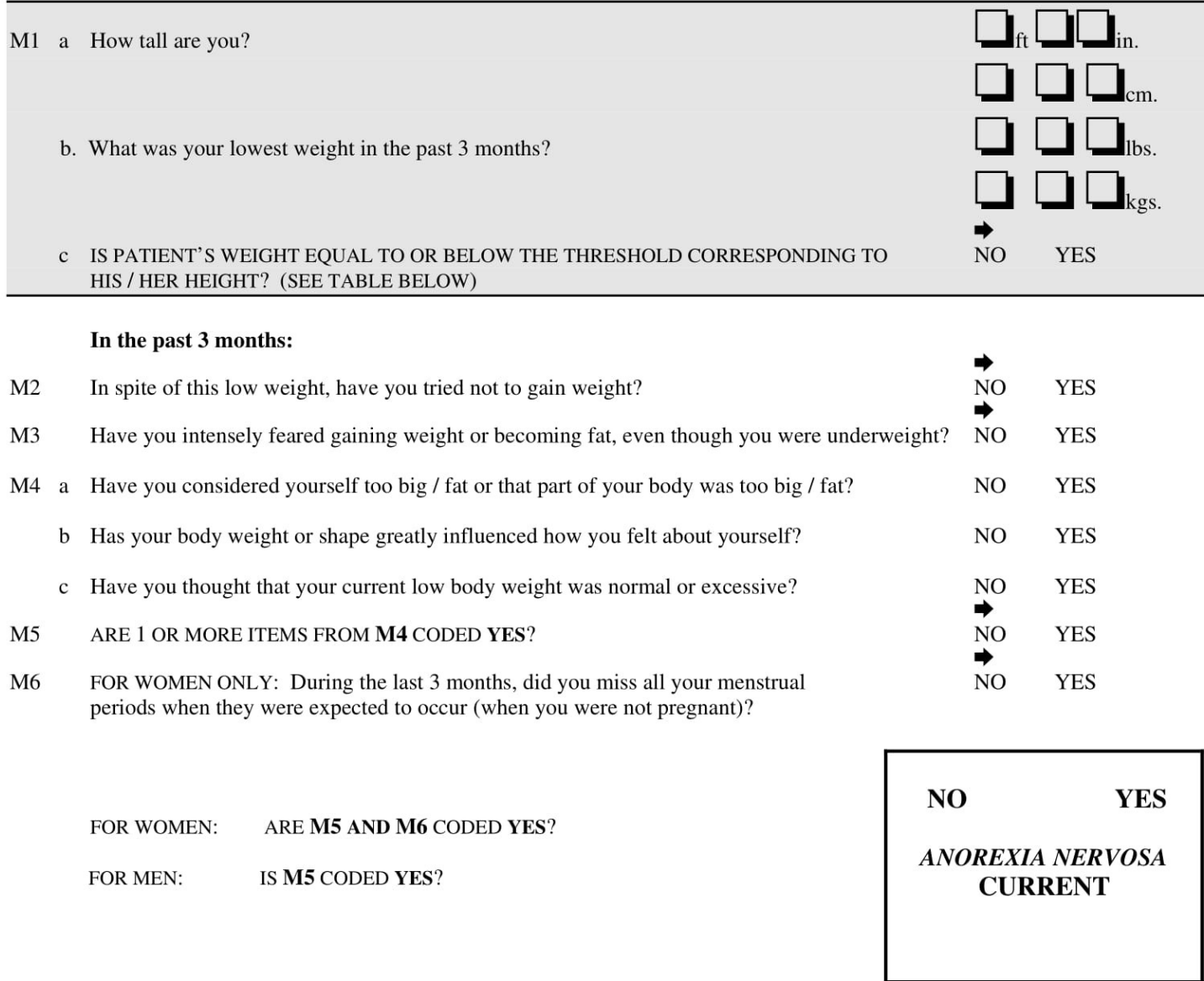

HEIGHT / WEIGHT TABLE CORRESPONDING TO A BMI THRESHOLD OF 17.5 KG/M²

\begin{tabular}{|c|c|c|c|c|c|c|c|c|c|c|c|c|c|c|}
\hline \multicolumn{15}{|c|}{ Height/Weight } \\
\hline $\mathrm{ft} / \mathrm{in}$ & $4^{\prime} 9$ & $4^{\prime} 10$ & $4^{\prime} 11$ & $5^{\prime} 0$ & $5^{\prime} 1$ & $5^{\prime} 2$ & $5^{\prime} 3$ & $5^{\prime} 4$ & $5^{\prime} 5$ & $5 ' 6$ & $5 ' 7$ & $5^{\prime} 8$ & $5^{\prime} 9$ & $5^{\prime} 10$ \\
\hline $\mathrm{cm}$ & 145 & 147 & 150 & 152 & 155 & 158 & 160 & 163 & 165 & 168 & 170 & 173 & 175 & 178 \\
\hline \multicolumn{15}{|c|}{ Height/Weight } \\
\hline $\mathrm{ft} / \mathrm{in}^{\circ}$ & $5^{\prime} 11$ & $6^{\prime} 0$ & $6^{\prime} 1$ & $6 ' 2$ & $6 ' 3$ & & & & & & & & & \\
\hline $\mathrm{kgs}$ & 57 & 59 & 60 & 62 & 64 & & & & & & & & & \\
\hline
\end{tabular}




\section{N. BULIMIA NERVOSA}

$\Leftrightarrow$ MEANS : GO TO THE DIAGNOSTIC BOXES, CIRCLE NO IN ALL DIAGNOSTIC BOXES, AND MOVE TO THE NEXT MODULE)

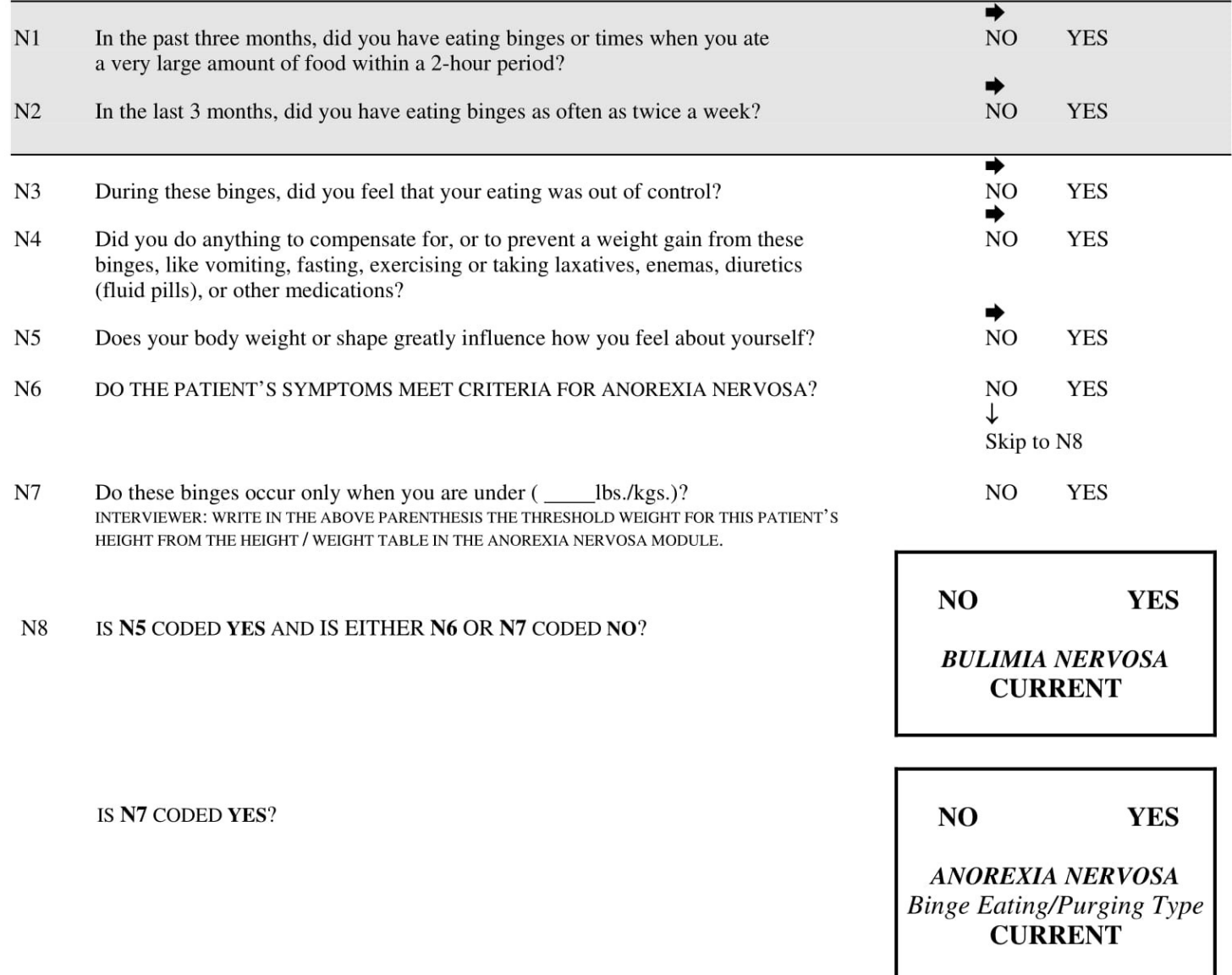




\section{O. GENERALIZED ANXIETY DISORDER}

$(\Rightarrow$ MEANS : GO TO THE DIAGNOSTIC BOX, CIRCLE NO, AND MOVE TO THE NEXT MODULE)

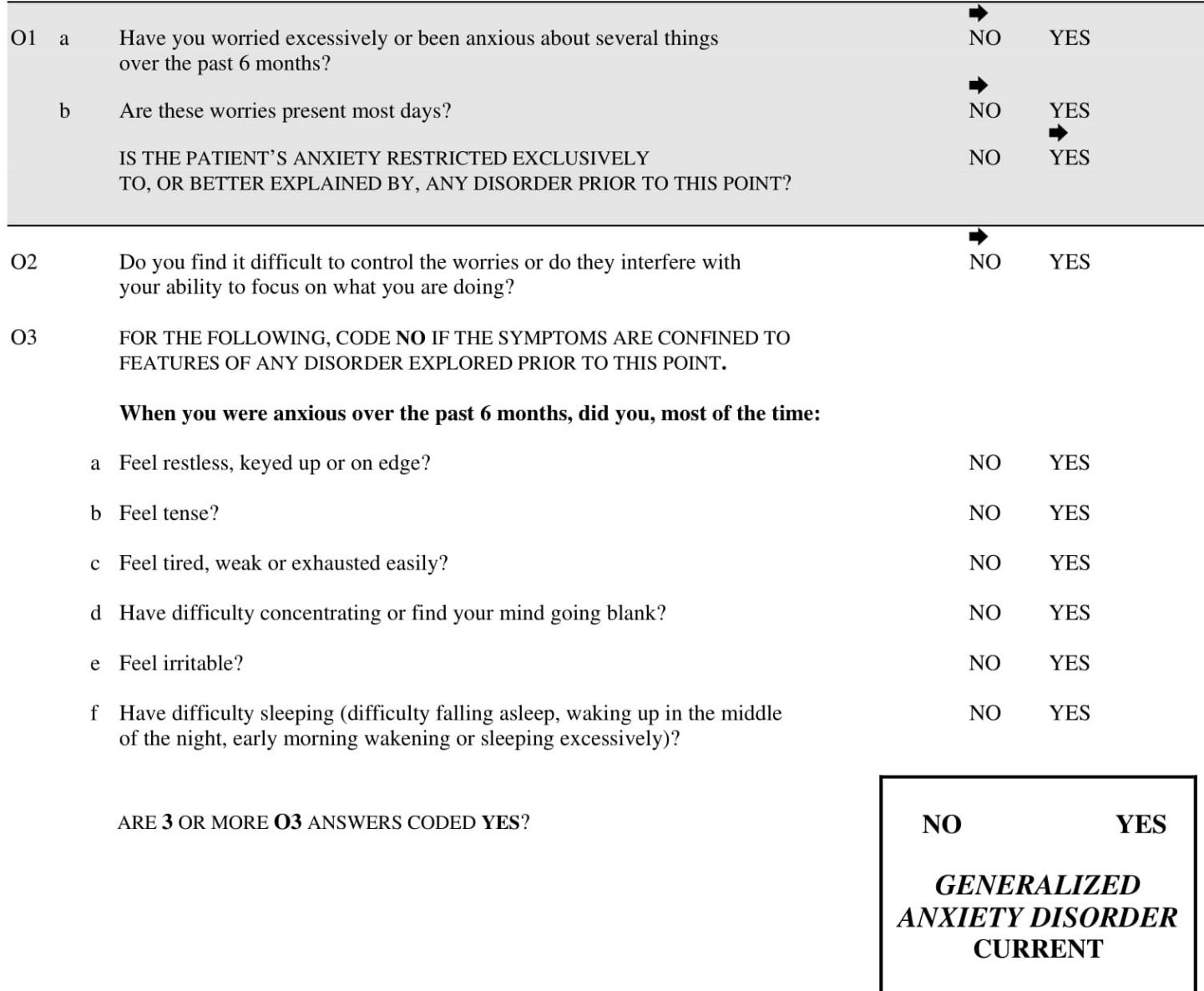




\section{P. ANTISOCIAL PERSONALITY DISORDER (optional)}

$(\rightarrow$ MEANS : GO TO THE DIAGNOSTIC BOX AND CIRCLE NO.)

P1

Before you were 15 years old, did you:

a repeatedly skip school or run away from home overnight?

NO YES

b repeatedly lie, cheat, "con" others, or steal?

NO YES

c start fights or bully, threaten, or intimidate others?

NO YES

d deliberately destroy things or start fires?

NO YES

e deliberately hurt animals or people?

NO YES

f force someone to have sex with you?

ARE 2 OR MORE P1 ANSWERS CODED YES?

DO NOT CODE YES TO THE BEHAVIORS BELOW IF THEY ARE EXCLUSIVELY POLITICALLY OR RELIGIOUSLY MOTIVATED.

Since you were 15 years old, have you:

a repeatedly behaved in a way that others would consider irresponsible, like failing to pay for things you owed, deliberately being impulsive or deliberately not working to support yourself?

b done things that are illegal even if you didn't get caught (for example, destroying property, shoplifting, stealing, selling drugs, or committing a felony)?

c been in physical fights repeatedly (including physical fights with your spouse or children)?

d often lied or "conned" other people to get money or pleasure, or lied just for fun?

e exposed others to danger without caring?

NO YES

f felt no guilt after hurting, mistreating, lying to, or stealing from others, or after damaging property?

ARE 3 OR MORE P2 QUESTIONS CODED YES?

NO

YES

ANTISOCIAL PERSONALITY

DISORDER

LIFETIME 


\section{REFERENCES}

Sheehan DV, Lecrubier Y, Harnett-Sheehan K, Janavs J, Weiller E, Bonara LI, Keskiner A, Schinka J, Knapp E, Sheehan MF, Dunbar GC. Reliability and Validity of the MINI International Neuropsychiatric Interview (M.I.N.I.): According to the SCID-P. European Psychiatry. 1997; $12: 232-241$.

Lecrubier Y, Sheehan D, Weiller E, Amorim P, Bonora I, Sheehan K, Janavs J, Dunbar G. The MINI International Neuropsychiatric Interview (M.I.N.I.) A Short Diagnostic Structured Interview: Reliability and Validity According to the CIDI. European Psychiatry. 1997; 12: $224-231$.

Sheehan DV, Lecrubier Y, Harnett-Sheehan K, Amorim P, Janavs J, Weiller E, Hergueta T, Baker R, Dunbar G: The Mini International Neuropsychiatric Interview (M.I.N.I.): The Development and Validation of a Structured Diagnostic Psychiatric Interview. J. Clin Psychiatry, 1998;59(suppl 20):22-33.

Amorim P, Lecrubier Y, Weiller E, Hergueta T, Sheehan D: DSM-III-R Psychotic Disorders: procedural validity of the Mini International Neuropsychiatric Interview (M.I.N.I.). Concordance and causes for discordance with the CIDI. European Psychiatry. 1998; $13: 26-34$.

\begin{tabular}{|c|c|c|}
\hline$\underline{\text { Translations }}$ & M.I.N.I. 4.4 or earlier versions & $\begin{array}{l}\text { M.I.N.I. 4.6/5.0, M.I.N.I. Plus 4.6/5.0 } \\
\text { and M.I.N.I. Screen 5.0: }\end{array}$ \\
\hline$\overline{\text { Afrikaans }}$ & R. Emsley & $\overline{\text { W. Maartens }}$ \\
\hline Arabic & & O. Osman, E. Al-Radi \\
\hline Bengali & & H. Banerjee, A. Banerjee \\
\hline \multicolumn{3}{|c|}{ Braille (English) } \\
\hline Brazilian Portuguese & P. Amorim & P. Amorim \\
\hline Bulgarian & L.G.. Hranov & \\
\hline Chinese & & $\begin{array}{l}\text { L. Carroll, Y-J. Lee, Y-S. Chen, C-C. Chen, C-Y. Liu, } \\
\text { C-K. Wu, H-S. Tang, K-D. Juang, Yan-Ping Zheng. }\end{array}$ \\
\hline Czech & & P. Zvlosky \\
\hline Danish & P. Bech & P. Bech, T. Schütze \\
\hline Dutch/Flemish & E. Griez, K. Shruers, T. Overbeek, K. Demyttenaere & I. Van Vliet, H. Leroy, H. van Megen \\
\hline English & $\begin{array}{l}\text { D. Sheehan, J. Janavs, R. Baker, K. Harnett-Sheehan, } \\
\text { E. Knapp, M. Sheehan }\end{array}$ & $\begin{array}{l}\text { D. Sheehan, R. Baker, J. Janavs, K. Harnett-Sheehan, } \\
\text { M. Sheehan }\end{array}$ \\
\hline Estonian & & J. Shlik, A. Aluoja, E. Khil \\
\hline Farsi/Persian & & K. Khooshabi, A. Zomorodi \\
\hline Finnish & M. Heikkinen, M. Lijeström, O. Tuominen & M. Heikkinen, M. Lijeström, O. Tuominen \\
\hline French & Y. Lecrubier, E. Weiller, I. Bonora, P. Amorim, J.P. Lepine & Y. Lecrubier, E. Weiller, P. Amorim, T. Hergueta \\
\hline German & I. v. Denffer, M. Ackenheil, R. Dietz-Bauer & G. Stotz, R. Dietz-Bauer, M. Ackenheil \\
\hline Greek & S. Beratis & T. Calligas, S. Beratis \\
\hline Gujarati & & M. Patel, B. Patel, Organon \\
\hline Hebrew & J. Zohar, Y. Sasson & R. Barda, I. Levinson, A. Aviv \\
\hline Hindi & & C. Mittal, K. Batra, S. Gambhir, Organon \\
\hline Hungarian & I. Bitter, J. Balazs & I. Bitter, J. Balazs \\
\hline Icelandic & & J.G. Stefansson \\
\hline Italian & $\begin{array}{l}\text { I. Bonora, L. Conti, M. Piccinelli, M. Tansella, G. Cassano, } \\
\text { Y. Lecrubier, P. Donda, E. Weiller }\end{array}$ & L. Conti, A. Rossi, P. Donda \\
\hline Japanese & & $\begin{array}{l}\text { T. Otsubo, H. Watanabe, H. Miyaoka, K. Kamijima, } \\
\text { J.Shinoda, K.Tanaka, Y. Okajima }\end{array}$ \\
\hline Kannada & & Organon \\
\hline Korean & & K.S. Oh and Korean Academy of Anxiety Disorders \\
\hline Latvian & V. Janavs, J. Janavs, I. Nagobads & V. Janavs, J. Janavs \\
\hline Lithuanian & & A. Bacevicius \\
\hline Malayalam & & Organon \\
\hline Marathi & & Organon \\
\hline Norwegian & G. Pedersen, S. Blomhoff & K.A. Leiknes , U. Malt, E. Malt, S. Leganger \\
\hline Polish & M. Masiak, E. Jasiak & M. Masiak, E. Jasiak \\
\hline Portuguese & P. Amorim & P. Amorim, T. Guterres \\
\hline Punjabi & & A. Gahunia, S. Gambhir \\
\hline Romanian & & O. Driga \\
\hline Russian & & $\begin{array}{l}\text { A. Bystritsky, E. Selivra, M. Bystritsky, L. Shumyak, } \\
\text { M. Klisinska. }\end{array}$ \\
\hline Serbian & I. Timotijevic & I. Timotijevic \\
\hline Setswana & & K. Ketlogetswe \\
\hline Slovenian & & M. Kocmur, M. Kocmur \\
\hline Spanish & L. Ferrando, J. Bobes-Garcia, J. Gilbert-Rahola, Y. Lecrubier & $\begin{array}{l}\text { L. Ferrando, L. Franco-Alfonso, M. Soto, J. Bobes- } \\
\text { Garcia, O. Soto, L. Franco, G. Heinze, C. Santana, } \\
\text { R. Hidalgo }\end{array}$ \\
\hline Swedish & M. Waern, S. Andersch, M. Humble & $\begin{array}{l}\text { C. Allgulander, H. Agren M. Waern, A. Brimse, } \\
\text { M. Humble. }\end{array}$ \\
\hline Tamil & & Organon \\
\hline Telugu & & Organon \\
\hline M.I.N.I. 5.0.0 (Ju & uly 1, 2006) & \\
\hline
\end{tabular}


Thai

Turkish T. Örnek, A. Keskiner, I. Vahip
P. Kittirattanapaiboon, S. Mahatnirunkul, P. Udomrat,

P. Silpakit,, M. Khamwongpin, S. Srikosai.

T. Örnek, A. Keskiner, A.Engeler

S. Gambhir

A validation study of this instrument was made possible, in part, by grants from SmithKline Beecham and the European Commission.

The authors are grateful to Dr. Pauline Powers for her advice on the modules on Anorexia Nervosa and Bulimia. 


\section{Appendix IV: Antidepressant Treatment History Form (ATHF)}

Harold A. Sackeim

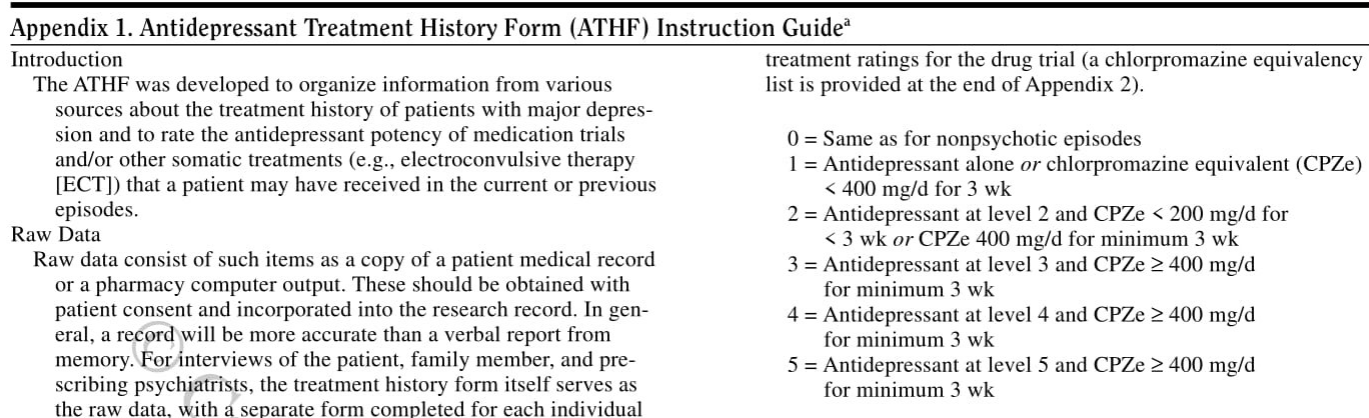

A separate Summary Form should be completed for each episode of major depression. Review all sources of information regarding each trial in making these determinations, giving greatest weight to medical documentation, blood levels, and multiple sources of confirmation. The start and stop dates for the period of the trial for which the patient is being rated (e.g., maintained oral dose or blood level for 4 weeks or greater) should be indicated, followed by the generic name(s) of the medication. Note explicitly combination trials and provide a separate rating for each agent in tricyclic antidepressant (TCA)/monoamine oxidase inhibitor, TCA/ selective serotonin reuptake inhibitor (SSRI), or other combinations. In rating relative antidepressant resistance, note that noncompliance or instances of good therapeutic response followed by rapid relapse in the absence of continuation therapy at adequate levels or due to noncompliance prevent rating a trial at level " 3 " or higher. For each trial, provide a global confidence score for the antidepressant resistance rating. This score should reflect the rater's certainty regarding dose, duration, compliance, and clinical outcome of the medication trial. For ECT trials, the confidence rating should reflect certainty regarding the number of ECT treatments given and the outcome of the treatment. At this time, confidence in reports of dosage of ECT is not being rated, and compliance with treatment is usually $100 \%$ (patient was present at the treatment). The scale to be used for this judgment is provided below: below:

1. No Confidence Rating: Discrepant or clearly unreliable information regarding dose, duration, compliance, and outcome of a medication trial or number and outcome of ECT trial.

2. Low Confidence Rating: Information is marginal. Evidence of contradictions in information or significant doubt exists regarding dose, duration, compliance, and outcome of a medication trial or the number of treatments and outcome of ECT trial.

3. Moderate Confidence Rating: Adequate information is available but based largely on one source that appears reliable. Areas of doubt not critical in medication or ECT resistance rating.

4. Strong Confidence Rating: Adequate information is available from more than one reliable source without significant discrepancy regarding dose, duration, compliance, and outcome of a medication trial or the number of treatments and outcome of ECT trial.

5. High Confidence Rating: Trial dose, duration, compliance, and outcome or the number of treatments and outcome of ECT trial confirmed by multiple sources, with excellent documentation (blood levels, medication orders), strong evidence of compliance, and outcome certain.

After the global confidence rating is made for the rating of relative medication or ECT resistance, specific confidence ratings should be made with respect to dose, duration, compliance, and outcome of the trials. The same 1 to 5 rating scale used for the global confidence rating should be applied to these specific ratings.

cont. 


\begin{tabular}{|c|c|}
\hline $\begin{array}{l}\text { Use of Rating Criteria } \\
\text { The tables shown in Appendix } 2 \text { provide specific criteria to be used } \\
\text { in rating the individual medication trials. These criteria are guides, } \\
\text { but any departure from their use must be justified and docu- } \\
\text { mented. The general principles to be followed are as follows: } \\
\text { (1) trials with a duration less than } 4 \text { weeks receive a score of "1," } \\
\text { independent of dosage; (2) monotherapy with medications without } \\
\text { established efficacy for unipolar depression receive a score of "1" } \\
\text { independent of dosage or duration (e.g., antipsychotics, benzo- } \\
\text { diazepines, sedatives, stimulants, thyroid hormones, repetitive } \\
\text { transcranial magnetic stimulation, herbal preparations), while for } \\
\text { other agents with uncertain efficacy the maximum score could be } \\
\text { "2" (alprazolam, specific anticonvulsants, lithium); (3) for selec- } \\
\text { tive heterocyclic antidepressants (HCAs), information regarding }\end{array}$ & $\begin{array}{l}\text { blood levels takes precedence over oral dosage; (4) evidence of } \\
\text { noncompliance diminishes the rating of trial strength (e.g., clearly } \\
\text { noncompliant patients should not receive a score of " } 3 \text {," the } \\
\text { threshold for resistance); (5) abandoning a trial because of side } \\
\text { effects in the context of significant clinical improvement also } \\
\text { diminishes the rating of trial strength; ( } 6 \text { ) for combination trials } \\
\text { (e.g., HCA and SSRI), each medication is rated separately. For all } \\
\text { patients, an exception is made for lithium augmentation. The rat- } \\
\text { ings for these trials are increased by one point if lithium was ad- } \\
\text { ministered for at least } 2 \text { weeks and the score for the antidepressant } \\
\text { met the threshold for an adequate trial (e.g., "3" or greater). In } \\
\text { psychotic patients, an exception is also made for concurrent treat- } \\
\text { ment with an antidepressant and antipsychotic (with or without } \\
\text { lithium). }\end{array}$ \\
\hline
\end{tabular}

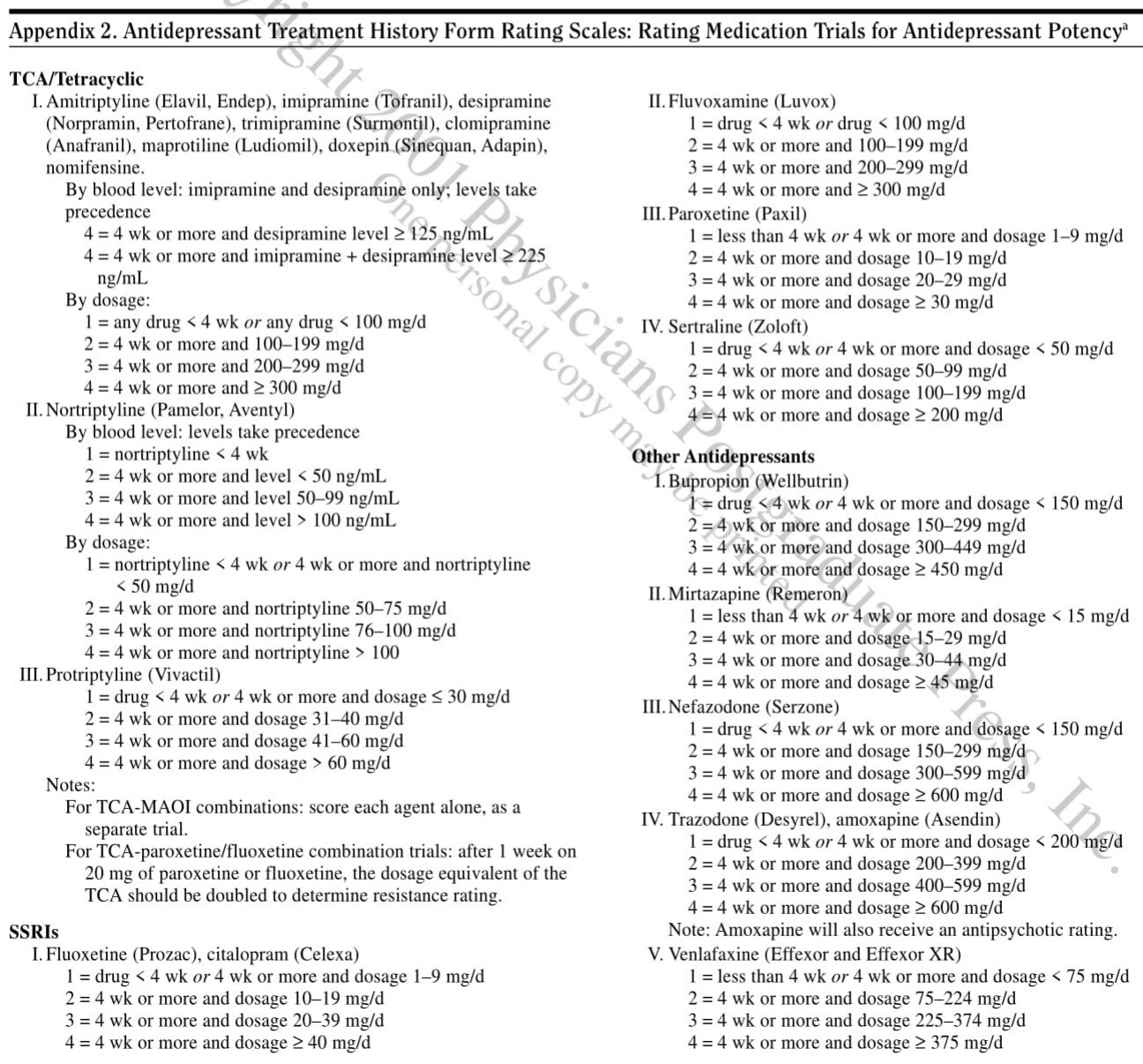




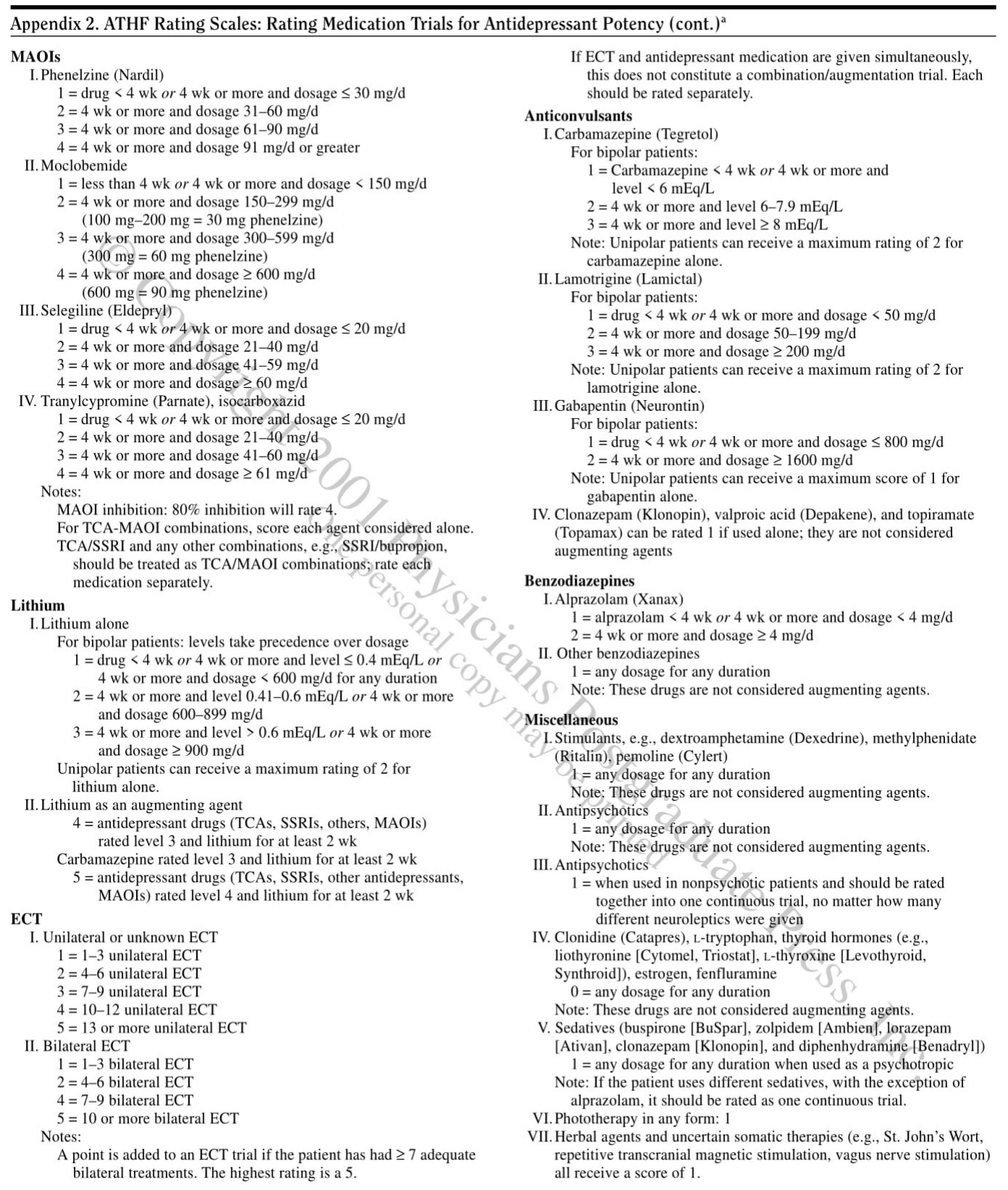


Appendix 2. ATHF Rating Scales: Rating Medication Trials for Antidepressant Potency (cont.) ${ }^{\mathrm{a}}$

\begin{tabular}{|c|c|c|c|}
\hline $\begin{array}{l}\text { Equivalent Doses of Antipsychotic } \\
\text { Generic name (U.S. trade name) }\end{array}$ & Drugs ${ }^{a}$ & quivalent Doses & \\
\hline Phenothiazines & & & \\
\hline Chlorpromazine (Thorazine) & $100 \mathrm{mg}$ & $200 \mathrm{mg}$ & $400 \mathrm{mg}$ \\
\hline Thioridazine & $100 \mathrm{mg}$ & $200 \mathrm{mg}$ & $400 \mathrm{mg}$ \\
\hline Mesoridazine (Serentil) & $50 \mathrm{mg}$ & $100 \mathrm{mg}$ & $200 \mathrm{mg}$ \\
\hline Trifluoperazine (Stelazine) & $4 \mathrm{mg}$ & $8 \mathrm{mg}$ & $16 \mathrm{mg}$ \\
\hline Fluphenazine & $1.5 \mathrm{mg}$ & $3 \mathrm{mg}$ & $6 \mathrm{mg}$ \\
\hline Fluphenazine decanoate & $0.25 \mathrm{~cm}^{3} / \mathrm{mo}$ & $0.5 \mathrm{~cm}^{3} / \mathrm{mo}$ & $1 \mathrm{~cm}^{3} / \mathrm{mo}$ \\
\hline Perphenazine (Trilafon, Etrafon) & $10 \mathrm{mg}$ & $20 \mathrm{mg}$ & $40 \mathrm{mg}$ \\
\hline Prochlorperazine (Compazine) & $15 \mathrm{mg}$ & $30 \mathrm{mg}$ & $60 \mathrm{mg}$ \\
\hline Thioxanthenes & & & \\
\hline Thiothixene (Navane) & $5 \mathrm{mg}$ & $10 \mathrm{mg}$ & $20 \mathrm{mg}$ \\
\hline Chlorprothixene (Taractan) & $50 \mathrm{mg}$ & $100 \mathrm{mg}$ & $200 \mathrm{mg}$ \\
\hline Butyrophenone & & & \\
\hline Haloperidol (Haldol) & $2 \mathrm{mg}$ & $4 \mathrm{mg}$ & $8 \mathrm{mg}$ \\
\hline Haloperidol decanoate & $\ldots$ & $0.25 \mathrm{~cm}^{3} / \mathrm{mo}$ & $0.5 \mathrm{~cm}^{3} / \mathrm{mo}$ \\
\hline Dibenzoxazepine & & & \\
\hline Loxapine (Loxitane) & $15 \mathrm{mg}$ & $30 \mathrm{mg}$ & $60 \mathrm{mg}$ \\
\hline Amoxapine (Asendin) & $125 \mathrm{mg}$ & $250 \mathrm{mg}$ & $500 \mathrm{mg}$ \\
\hline Dibenzepine & & & \\
\hline Clozapine (Clozaril) & $60 \mathrm{mg}$ & $120 \mathrm{mg}$ & $240 \mathrm{mg}$ \\
\hline Dihydroindolone & & & \\
\hline Molindone (Moban) & $10 \mathrm{mg}$ & $20 \mathrm{mg}$ & $40 \mathrm{mg}$ \\
\hline Diphenylbutylpiperidine & & & \\
\hline Pimozide (Orap) & $2 \mathrm{mg}$ & $4 \mathrm{mg}$ & $8 \mathrm{mg}$ \\
\hline Other atypical antipsychoti & & & \\
\hline Risperidone (Risperdal) & $1.5 \mathrm{mg}$ & $3 \mathrm{mg}$ & $6 \mathrm{mg}$ \\
\hline Sulpiride & $300 \mathrm{mg}$ & $600 \mathrm{mg}$ & $1200 \mathrm{mg}$ \\
\hline Olanzapine (Zyprexa) & & $10 \mathrm{mg}$ & $20 \mathrm{mg}$ \\
\hline Quetiapine (Seroquel) & & $200 \mathrm{mg}$ & $400 \mathrm{mg}$ \\
\hline
\end{tabular}

"Trade names shown parenthetically. Abbreviations: ATHF = Antidepressant Treatment History Form, ECT = electroconvulsive therapy, MAOI = monoamine oxidase inhibitor, $\mathrm{SSRI}=$ selective serotonin reuptake inhibitor, $\mathrm{TCA}=$ tricyclic antidepressant, $\mathrm{XR}=$ extended release .

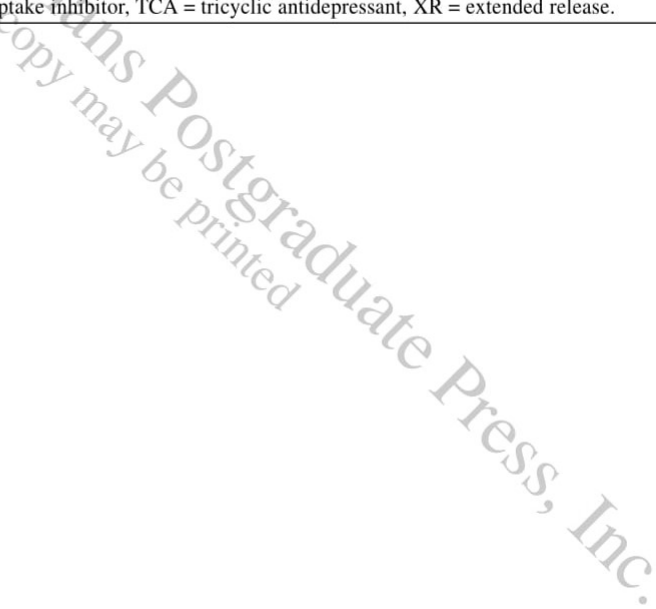




\section{Appendix V: Montgomery-Åsberg Depression Rating Scale (MADRS)}

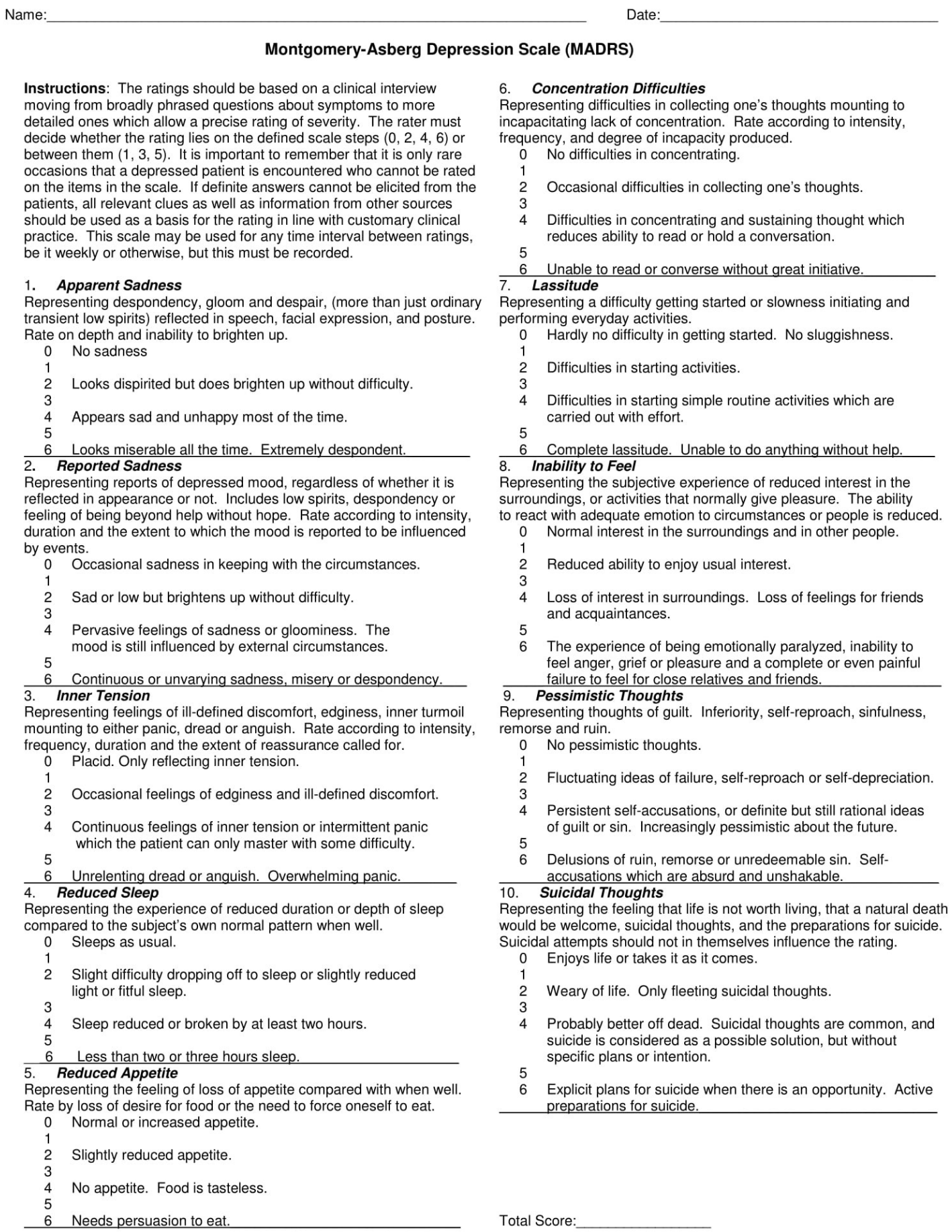

Copyright notice: The Montgomery-Asberg Depression Scale (MADRS) is copyrighted by Stuart Montgomery, M.D. Permission has been granted by the author to reproduce the scale on this website for clinicians to use in their practice and for researchers to use in non-industry studies. For other uses of the scale, the owner of the copyright should be contacted at stuart@samontgomery.co.uk. 


\title{
Appendix VI: Brief Psychiatric Rating Scale-Positive Symptoms subscale (BPRS-P)
}

\author{
CLIENT NAME: \\ CLIENT ID\#:
}

DATE:

MD:

\section{BRIEF PSYCHIATRIC RATING SCALE (BPRS)}

Please enter the score for the term which best describes the patient's condition.

$0=$ not assessed, $1=$ not present, $2=$ very mild, $3=$ mild, $4=$ moderate, $5=$ moderately severe, $6=$ severe, $7=$ extremely severe

\begin{tabular}{|c|c|c|c|}
\hline $\begin{array}{l}\text { 1. SOMATIC CONCERN } \\
\text { Degree of concern over present bodily health. Rate the } \\
\text { degree to which physical health is perceived as a problem } \\
\text { by the patient, whether complaints have a realistic basis or } \\
\text { not. }\end{array}$ & SCORE & $\begin{array}{l}\text { 10. HOSTILITY } \\
\text { Animosity, contempt, belligerence, disdain for other people } \\
\text { outside the interview situation. Rate solely on the basis of } \\
\text { the verbal report of feelings and actions of the patient } \\
\text { toward others; do not infer hostility from neurotic defenses, } \\
\text { anxiety, nor somatic complaints. (Rate attitude toward } \\
\text { interviewer under "uncooperativeness"). }\end{array}$ & SCORE \\
\hline $\begin{array}{l}\text { 2. ANXIETY } \\
\text { Worry, fear, or over-concern for present or future. Rate } \\
\text { solely on the basis of verbal report of patient's own } \\
\text { subjective experiences. Do not infer anxiety from physical } \\
\text { signs or from neurotic defense mechanisms. }\end{array}$ & SCORE & $\begin{array}{l}\text { 11. SUSPICIOUSNESS } \\
\text { Brief (delusional or otherwise) that others have now, or } \\
\text { have had in the past, malicious or discriminatory intent } \\
\text { toward the patient. On the basis of verbal report, rate only } \\
\text { those suspicions which are currently held whether they } \\
\text { concern past or present circumstances. }\end{array}$ & SCORE \\
\hline $\begin{array}{l}\text { 3. EMOTIONAL WITHDRAWAL } \\
\text { Deficiency in relating to the interviewer and to the } \\
\text { interviewer situation. Rate only the degree to which the } \\
\text { patient gives the impression of failing to be in emotional } \\
\text { contact with other people in the interview situation. }\end{array}$ & SCORE & $\begin{array}{l}\text { 12. HALLUCINATORY BEHAVIOR } \\
\text { Perceptions without normal external stimulus } \\
\text { correspondence. Rate only those experiences which are } \\
\text { reported to have occurred within the last week and which } \\
\text { are described as distinctly different from the thought and } \\
\text { imagery processes of normal people. }\end{array}$ & SCORE \\
\hline $\begin{array}{l}\text { 4. CONCEPTUAL DISORGANIZATION } \\
\text { Degree to which the thought processes are confused, } \\
\text { disconnected, or disorganized. Rate on the basis of } \\
\text { integration of the verbal products of the patient; do not rate } \\
\text { on the basis of patient's subjective impression of his own } \\
\text { level of functioning. }\end{array}$ & SCORE & $\begin{array}{l}\text { 13. MOTOR RETARDATION } \\
\text { Reduction in energy level evidenced in slowed movements. } \\
\text { Rate on the basis of observed behavior of the patient only; } \\
\text { do not rate on the basis of patient's subjective impression } \\
\text { of own energy level. }\end{array}$ & SCORE \\
\hline $\begin{array}{l}\text { 5. GUILT FEELINGS } \\
\text { Over-concern or remorse for past behavior. Rate on the } \\
\text { basis of the patient's subjective experiences of guilt as } \\
\text { evidenced by verbal report with appropriate affect; do not } \\
\text { infer guilt feelings from depression, anxiety or neurotic } \\
\text { defenses. }\end{array}$ & SCORE & $\begin{array}{l}\text { 14. UNCOOPERATIVENESS } \\
\text { Evidence of resistance, unfriendliness, resentment, and } \\
\text { lack of readiness to cooperate with the interviewer. Rate } \\
\text { only on the basis of the patient's attitude and responses to } \\
\text { the interviewer and the interview situation; do not rate on } \\
\text { basis of reported resentment or uncooperativeness outside } \\
\text { the interview situation. }\end{array}$ & SCORE \\
\hline $\begin{array}{l}\text { 6. TENSION } \\
\text { Physical and motor manifestations of tension "nervousness", } \\
\text { and heightened activation level. Tension should be rated } \\
\text { solely on the basis of physical signs and motor behavior and } \\
\text { not on the basis of subjective experiences of tension } \\
\text { reported by the patient. }\end{array}$ & SCORE & $\begin{array}{l}\text { 15. UNUSUAL THOUGHT CONTENT } \\
\text { Unusual, odd, strange or bizarre thought content. Rate } \\
\text { here the degree of unusualness, not the degree of } \\
\text { disorganization of thought processes. }\end{array}$ & SCORE \\
\hline $\begin{array}{l}\text { 7. MANNERISMS AND POSTURING } \\
\text { Unusual and unnatural motor benavior, the type of motor } \\
\text { behavior which causes certain mental patients to stand out } \\
\text { in a crowd of normal people. Rate only abnormality of } \\
\text { movements; do not rate simple heightened motor activity } \\
\text { here. }\end{array}$ & SCORE & $\begin{array}{l}\text { 16. BLUNTED AFFECT } \\
\text { Reduced emotional tone, apparent lack of normal feeling or } \\
\text { involvement. }\end{array}$ & SCORE \\
\hline $\begin{array}{l}\text { 8. GRANDIOSITY } \\
\text { Exaggerated self-opinion, conviction of unusual ability or } \\
\text { powers. Rate only on the basis of patient's statements about } \\
\text { himself or self-in-relation-to-others, not on the basis of his } \\
\text { demeanor in the interview situation. }\end{array}$ & SCORE & $\begin{array}{l}\text { 17. EXCITEMENT } \\
\text { Heightened emotional tone, agitation, increased reactivity. }\end{array}$ & SCORE \\
\hline $\begin{array}{l}\text { 9. DEPRESSIVE MOOD } \\
\text { Despondency in mood, sadness. Rate only degree of } \\
\text { despondency; do not rate on the basis of inferences } \\
\text { concerning depression based upon general retardation and } \\
\text { somatic complaints. }\end{array}$ & SCORE & $\begin{array}{l}\text { 18. DISORIENTATION } \\
\text { Confusion or lack of proper association for person, place or } \\
\text { time. }\end{array}$ & SCORE \\
\hline
\end{tabular}

